FINAL REPORT

FHWA/IN/JTRP-2005/9

\title{
AN ASSESSMENT OF HIGHWAY FINANCING NEEDS IN INDIANA
}

\author{
By \\ Kumares C. Sinha \\ Olson Distinguished Professor \\ Samuel Labi \\ Visiting Assistant Professor \\ Stacey Hodge \\ Graduate Research Assistant \\ Gabriel Tine \\ Graduate Research Assistant \\ and \\ Hardik Shah \\ Graduate Research Assistant \\ School of Civil Engineering \\ Purdue University \\ Joint Transportation Research Program \\ Project No. C-36-73Y \\ File No.: 3-4-25 \\ SPR-2637 \\ Prepared in Cooperation with the \\ Indiana Department of Transportation and \\ The U.S. Department of Transportation \\ Federal Highway Administration
}

The contents of this report reflect the views of the authors who are responsible for the facts and the accuracy of the data presented herein. The contents do not necessarily reflect the official views of the Federal Highway Administration and the Indiana Department of Transportation. The report does not constitute a standard, a specification, or a regulation.

Purdue University

West Lafayette, Indiana, 47907

June 2005 


\section{TECHNICAL Summary}

INDOT Research

Technology Transfer and Project Implementation Information

TRB Subject Code: 14-1 Financing Sources

Publication No.: FHWA/IN/JTRP-2005/9, SPR-2637

June 2005

Final Report

\section{An Assessment of Highway Financing Needs in Indiana}

\section{Introduction}

In order to facilitate informed fiscal planning, it is necessary to reliably assess the current and future trends in highway financing needs both at the state and local levels vis-à-vis revenue projections and to identify any future gaps in financing. This report provides a methodology for state and local agencies to track past, current and future needs for highway funds and expected revenue levels under a range of scenarios. Using such a methodology, shortterm and long-term financing plans can be developed both for state and local highway networks. The need was assessed for a 20062020 horizon period.

\section{Findings}

The needs for highway infrastructure preservation and capital improvements were primarily estimated by considering the current and projected road and bridge conditions. Capital needs were estimated using existing long-range plans. The financing needs for the state highway agency are about 30 billion dollars (2002\$) for the 15-year period of 20062020. Local highway needs during the same period are about $\$ 29$ billion.

Long-term forecasts of highway revenues by revenue source were developed. The forecasting model provides state and local shares of state generated revenues year by year for the 15-year period. On the basis of the model results, the 15-year revenues for the state agency from state- generated sources would be about $\$ 10$ billion while the local share would be about $\$ 7$ billion. Using the best possible estimate of the Federal aid, the state highway revenue is expected to be about $\$ 16.87$ billion. The resulting revenue gap for the state highway agency would then be $\$ 12.93$ to $\$ 13.33$ billion for the 15 -year period or $\$ 0.86$ to $\$ 0.89$ billion annually. The projected revenue for local agencies was found to be about \$13.8 to $\$ 15.7$ billion for the 15-year period. The total resulting shortfall ranges from $\$ 12.4$ to $\$ 14.7$ billion or $\$ 0.83$ to $\$ 0.98$ billion annually.

\section{Implementation}

Policymakers at the state and local levels can use the results of this study for fiscal planning and budgeting. The recommendations for raising revenues presented can be a starting point for further exploration to address the funding gap identified. The present study addressed the 15-year highway financing needs for the state of Indiana, and in the process identified a number of areas that could be investigated at a future date. Future research may seek to expand the present study to answer the question of what type of user tax would be equitable and politically acceptable to taxpayers in Indiana. 


\section{Contacts}

For more information:

Prof. Kumares C. Sinha

Principal Investigator

School of Civil Engineering

Purdue University

West Lafayette, IN 47907-2051

Phone: (765) 494-2211

Fax: (765) 496-7996

E-mail: sinha@ecn.purdue.edu
Indiana Department of Transportation

Division of Research

1205 Montgomery Street

P.O. Box 2279

West Lafayette, IN 47906

Phone: (765) 463-1521

Fax: (765) 497-1665

\section{Purdue University}

Joint Transportation Research Program

School of Civil Engineering

West Lafayette, IN 47907-1284

Phone: (765) 494-9310

Fax: (765) 496-7996

E:mail: jtrp@ecn.purdue.edu

http://www.purdue.edu/jtrp 
TECHNICAL REPORT STANDARD TITLE PAGE

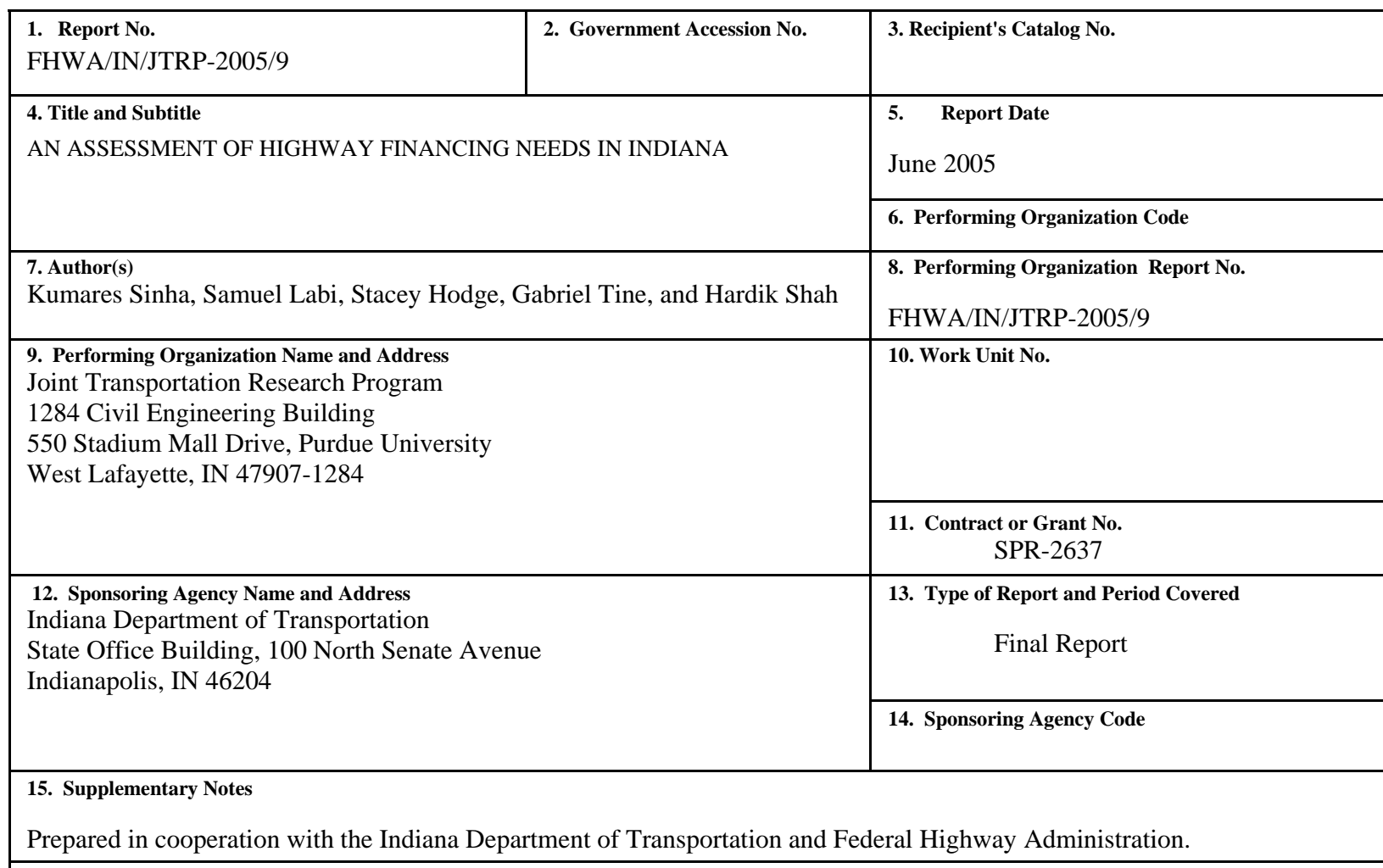

\section{Abstract}

In recent years highway revenues in Indiana have been eroding due to inflation, increased costs, and the increased use of fuel-efficient vehicles. It is necessary, therefore, to reliably assess the current and future needs for highway funding in order to facilitate informed fiscal planning. This study provides a methodology for tracking current and future demand for highway financing, along with a systematic modeling approach for revenue projections such that financing plans can be developed for state and local highway networks. Highway infrastructure needs for pavements, bridges, safety, and capacity improvements for the 15-year period between 2006 and 2020 are assessed for both state and local agencies. Needs are primarily determined on the basis of pavement and bridge conditions. Revenue projections are made using the current allocation and funding mechanisms. The study results indicate that the 15-year financing needs for the state highway agency are in the range of $\$ 30$ billion (2002\$) and the total expected revenue during the same period is $\$ 16.87$ billion, creating a financing gap in the range of $\$ 0.86$ to $\$ 0.89$ billion per year. The local agencies will face a revenue gap in the range of $\$ 0.83$ to $\$ 0.98$ million annually during the same period.

\section{Key Words}

Highway Finance, Highway Need Assessment, HERS-ST, Funding Gap, Revenue Forecasting Methodology, State Highway Revenue, Local Highway Revenue, Motor Fuel Taxes and Registration.

\section{Distribution Statement}

No restrictions. This document is available to the public through the National Technical Information Service, Springfield, VA 22161

\begin{tabular}{|c|c|c|}
\hline $\begin{array}{c}\text { 19. Security Classif. (of this report) } \\
\text { Unclassified }\end{array}$ & $\begin{array}{c}\text { 20. Security Classif. (of this page) } \\
\text { 22. Price }\end{array}$ & Unclassified \\
\hline
\end{tabular}

Form DOT F 1700.7 (8-69) 


\section{ACKNOWLEDGMENTS}

The authors hereby acknowledge the contributions and support provided by the following

persons within the Indiana Department of Transportation (INDOT: Gary Eaton, Steve Fanning, William Flora, Mitch Hansel, David Holtz, Andy Jackson, Geraldine Lampley, Laurie Maudlin, Roy Nunnally, Stephen Smith, Leah Snow, and Rick Whitney. The authors would also like to thank Darren Timothy and David Winter for their help with the HERS-ST. The assistance of Tom Martin of Indiana LTAP is also acknowledged. 


\section{TABLE OF CONTENTS}

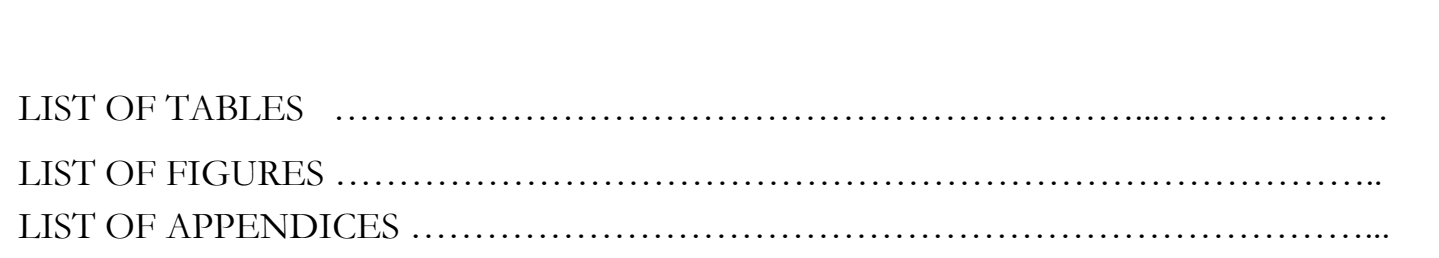

viii

xii

xiv

\section{CHAPTER 1 INTRODUCTION}

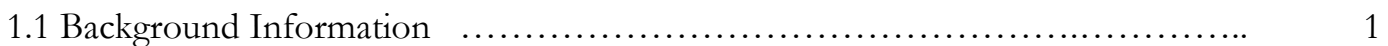

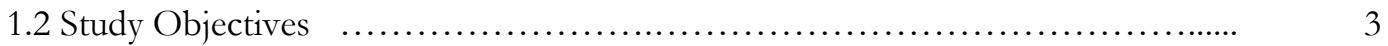

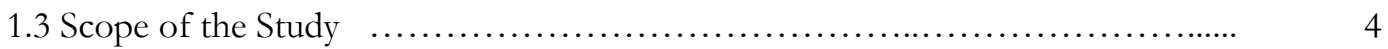

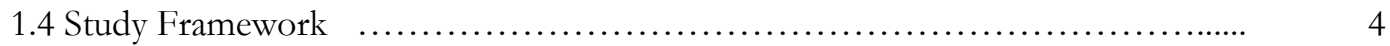

1.4.1 Highway Infrastructure Needs Assessment........................ 5

1.4.2 Revenue Analysis.......................................... 6

CHAPTER 2 NEEDS ASSESSMENT

2.1 Estimation of Physical Needs for State Highway Infrastructure Network....... 8

2.1.1 INDOT 2000-2025 Long Range Plan Projects..................... 10

2.1.2 New Bridges and Roads in Long Range Plan....................... 13

2.1.3 Pavement Condition and Design Life........................... 15

2.1.4 Pavement Treatments...................................... 18

2.1.4.1 Preventive Maintenance Treatments..................... 18

2.1.4.2 Pavement Rehabilitation Partial 3-R.................... 19

2.1.4.3 Pavement Replacement............................... 19

2.1.5 Costs of Highway Repairs................................... $\quad 20$

2.1.5.1 HERS-ST Analysis Treatment Costs...................... $\quad 20$

2.1.5.2 Routine Maintenance Costs........................... 23

2.1.6 HERS-ST Physical Needs Analysis................................ 24

2.1.6.1 Collection of Data for HERS-ST......................... 25

2.1.6.2 HERS-ST Methodology............................... 26

2.1.6.3 HERS-ST Scenarios................................ $\quad 30$

2.1.6.4 HERS-ST Analysis and Results....................... $\quad 30$

2.1.6.5 HERS-ST Summary and Conclusions................... 37

2.1.7 Manual Network Condition Analysis............................ 38

2.2 Estimation of Needs for Local Highway Network.......................... 67 
2.2.1 Local Road Inventory....

2.2.1.1 Approach using Need/Disbursements Ratio and TimeBased Strategies

2.2.1.2 Estimation of "Backlog” Using the Trigger Value Approach (Paved Road) ..............................

2.2.1.3 Estimation of "Backlog" Using the Annual Average Maintenance Expenditure (AAMEX) Approach (Unpaved Road) ....................................

2.2.1.4 Summary of Backlog Assessment for Local Roads and Pavement...........................................

2.2.1.5 Summary of Pavements Needs Assessment.

2.2.2 Need Assessment for Local Bridges....

2.2.2.1 Need Assessment on Basis of Sufficiency Ratings (Assessment of Backlog).

2.2.2.2 Need Assessment on Basis of Age (Assessment of Total Needs).

2.2.2.3 Summary of Need Assessment for Local Bridges....

2.2.3 Assessment of Overhead Costs and Capacity Needs

2.3 Total Needs for the State and Local Highway Network

\section{CHAPTER 3 ESTIMATION OF REVENUES}

3.1 Estimation of State Highway Revenues................................. 97

3.1.1 State Highway Funding.................................... 97

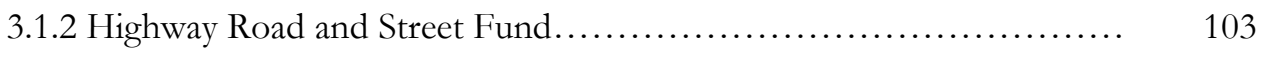

3.1.3 Motor Vehicle Highway Account............................... 104

3.1.4 Vehicle Registration and Licensing Fees.......................... 108

3.1.5 Description of Tax Types................................... 109

3.1.6 Special Distribution Account............................... 115

3.1.7 Debt Service Accounts.................................... 116

3.1.8 International Registration Program.......................... 116

3.2 Revenue Projections from Historical Trends for State Highway Agency......... 117

3.3 Historical Trends in Revenues for Local Agencies.......................... 120

3.3.1 Motor Vehicle Highway Account (MVHA)..................... 122

3.3.2 Local Road and Street Fund (LRSF) ......................... 124

3.3.3 Local Supplemental Funding............................... 125

3.3.4 Federal Aid............................................. 127

3.3.5 Dedicated Sources of Revenue for Local Bridges................... 129 
3.3.5.1 Cumulative Bridge Funds.............................. 129

Page

3.3.5.2 Major Bridge Funds............................... 129

3.3.5.3 Federal Aid for Bridges............................. $\quad 130$

3.3.6 Revenue Projections for Local Agencies.......................... 130

3.3.6.1 Local Revenue Projection Based on MVHA and LRSF....... 130

3.3.6.2 Projection of Federal Aid Revenue........................ 133

3.4 Highway Revenue Forecasting.................................... 135

3.4.1 Highway Revenue Forecasting Methodology........................ 135

3.4.2 Highway Revenue Forecasting Models........................... 135

3.4.3 Data Requirement for Revenue Forecasting and its Use............. 140

3.4.4 Vehicle Registration Forecast Equations........................... 143

3.4.5 Vehicle Miles of Travel Forecast Equations....................... 145

3.4.6 Vehicle Fleet Fuel Efficiency Determination....................... 148

3.4.7 Fuel Consumption Estimation................................. 149

3.4.8 Highway Revenue Estimation................................ 150

3.4.9 Highway Revenue Disbursement............................ 154

3.4.10 Validation of the Model Results............................ 156

3.4.11 Sensitivity of the Forecasts................................. 157

\section{CHAPTER 4 GAP ANALYSIS FOR STATE AND LOCAL AGENCIES}

4.1 State Highway Funding Gap Analysis.................................. 158

4.2 Local Highway Funding Gap Analysis................................... 160

4.3 Gap Reduction Scenarios............................................ 161

4.3.1 Increase in State Gasoline Tax............................... 161

4.3.2 Increase in State Diesel Tax.................................. 161

4.3.3 Combination of Gasoline and Diesel Tax Increases................. 162

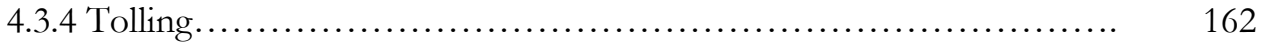

4.3.5 Gap Reduction Scenarios for Local Roads........................ 162

4.3.5.1 Local Fuel Taxes..................................... 163

4.3.5.2 Local Vehicle Taxes.................................. 163

4.3.5.3 Local Option Highway User Tax (LOHUT)................ 163

4.3.5.4 Property Taxes........................................ 165

4.3.5.5 Sales Taxes........................................... 165

4.3.5.6 Income, Payroll, and Employer Taxes................... 166 
4.3.5.7 Other Taxes......................................... 166

Page

\section{CHAPTER 5 SUMMARY AND CONCLUSIONS}

5.1 Summary of Findings.................................................. 167

5.2 Implementation of Study Results.................................... 168

5.3 Future Work........................................................ 168 


\section{LIST OF TABLES}

Table 
2.32 Fifteen-year Pavement Condition Preservation Needs Scenario B

2.33 Fifteen-year Pavement Condition Preservation Needs for Interstate Addedlanes, Scenario A

2.34 Fifteen-year Pavement Condition Preservation Needs for Non-Interstate Added-lanes, Scenario A

2.35 Fifteen-year Pavement Condition Preservation Needs for Interstate Addedlanes, Scenario B

2.36 Fifteen-year Pavement Condition Preservation Needs for Non-Interstate Added-lanes, Scenario B

2.37 Fifteen-year Preservation Needs for New Road Cosntruction

$2.38 \quad$ Bridge Work Activity Groups 61

2.39 Summary of Bridge Needs Assessment 2006-2020 62

2.40 Bridge Needs Assessment 2006-2020 63

2.41 State Highway Infrastructure Expenditures 1995-2003 64

2.42 Fifteen-year State Highway Needs, HERS-ST Method 66

$2.43 \quad$ Fifteen-year State Highway Needs, Manual Analysis Method 67

2.44 Methods Used for Local System Needs Assessment 68

2.45 Classification of Local Roads by Surface Type 69

2.46 Unit Costs Per Mile for Unpaved Roads Maintenance in Year $1983 \quad 70$

2.47 Estimated Needs for Preservation and Maintenance of Unpaved Roads in Sample Counties in 1998 and 2000 (\$1000)

2.48 Estimated Needs for Preservation and Maintenance of Paved Roads in Sample Counties in 1998 and 2000 (\$1000)

Maintenance Strategy 1 (\$Year 2000)

2.49 Estimated Needs for Preservation and Maintenance of Paved Roads in Sample Counties in 1998 and 2000 (\$1000)

Maintenance Strategy 2 (\$Year 2000)

2.50 Net Maintenance and Repair/Construction and Reconstruction

Disbursements in Sample Counties in 1998 (\$1000 in \$ Year 2002)

2.51 Net Maintenance and Repair/Construction and Reconstruction

Disbursements in Sample Counties in 2000 (\$1000 in \$ Year 2002)

2.52 Difference between Disbursements and Needs in 1998 (\$1000) Maintenance Strategy 1 (\$Year 2002) 
2.53 Difference between Disbursements and Needs in 2000 (\$1000) Maintenance Strategy 1 (\$ Year 2002)

2.54 Difference between Disbursements and Needs in 1998 (\$1000) Maintenance Strategy 2 (\$ Year 2002)

2.55 Difference between Disbursements and Needs in 2000 (\$1000) Maintenance Strategy 2 (\$Year 2002)

2.56 Estimated Fiscal Needs for Local Roads (\$ Year 2002)

2.57 Pavement Condition and IRI Values

2.58 IRI Threshold Value Corresponding to Level of Maintenance for Local Pavements

2.59 Backlog Pavement Preservation Needs for Rural Roads (2001)

2.60 Backlog Pavement Preservation Needs for Urban Roads (\$ Year 2002)

2.61 Backlog Preservation Needs for Local Roads 84

2.62 Summary of Pavement Needs for Indiana Local Roads (2006-2020) 84

2.63 Summary of Local Bridges Sufficiency Ratings (2000-2001) 85

2.64 Costs Estimations for Bridge Replacement (2000-2001) 86

2.65 Costs Estimations for Bridge Rehabilitation (2000-2001) 86

2.66 Age-based Physical Needs Assessment (NBIS 2000-2001) 88

2.67 Age-based Monetary Needs for Bridge Replacement (\$Year 2002) 89

2.68 Age-based Monetary Needs for Bridge Rehabilitation (\$ Year 2002) 91

2.69 Summary of Local Bridge Needs (2006-2020) (\$ Year 2002) 93

2.70 Local Overhead Costs as a Percentage of MVHA Receipts (Sample 8 Counties) (\$Year 2002)

2.71 Estimation of Capacity Needs for Indiana Local Roads (\$ Year 2002) 95

2.72 15-yr State Highway Needs (2006-2020) 95

2.73 15-yr Local Highway Needs (2006-2020) 96

3.1 State Generated Highway Revenue FY 2002 (Millions) 100

3.2 State Highway Net Expenses FY 2002 (Millions) 101

3.3 MVHA Historical Revenue 1995-1999 (Current \$) 107

3.4 MVHA Historical Revenue 2000-2003 (Current \$) 108

3.5 History of Indiana Fuel Tax Rates 1923-2003 (cents/gallon) 109

3.6 Fuel Tax Rates in Indiana and Neighboring States (cents/gallon) 110 
3.7 Inflation Impact of Highway Revenue (Millions)

Table

Page

3.8 State Highway Revenue History 1995-1998 (Millions, Current \$) 117

3.9 State Highway Revenue History 1999-2003 (Millions, Current \$) 117

$3.10 \quad$ Inflation Impact of Highway Revenue (Millions) 119

3.11 15-year Projected Revenue for State Highway Agency on the Basis of Historical Trend

3.12 MVHA Revenue for Indiana Local Agencies (1990-2000 FY) 124

3.13 HRSF Revenue for Indiana Local Agencies (1990-2000 FY) 125

3.14 Summary of Federal Aid Distributions for Local Use by Fund (5-Year

$3.152001(\mathrm{CY})$ County Major Bridge Fund Revenues 130

$3.16 \quad$ MVHA and LRSF Revenue Projection 133

3.172001 Supplemental Revenue for Indiana Local Agencies 134

3.18 Data Source for the Registration Related Revenue 141

3.19 Date Source for the Motor Fuel Related Revenue 142

$3.20 \quad$ VMT Predictions 147

3.21 Estimated Fleet Fuel Efficiency (FFE) Values for Automobiles, Light Duty Trucks and Tractors (miles per gallon) 149

3.22 Highway Revenue Forecasts for Registration Related Revenues (2006-2020) 152

$3.23 \quad$ Forecasts for Fuel Tax Revenues (2006-2020) 153

3.24 Total Highway Revenue Forecasts for the Period (2006-2020) 153

3.25 Disbursement of Revenues in Major Highway Accounts 155

3.26 Validation of the Model Using Actual Registration Revenue for Years 2001 and 2002 (2002 \$ value in Millions)

3.27 Validation of the Model Using Actual Motor Fuel Revenues for Years 2001 and 2002 (2002 \$ value in Millions) 156

4.1 Fifteen-year State Agency Funding Gap Using HERS-ST Analysis (in Billions of $2002 \$)$

4.2 Fifteen-year Local Agencies Funding Gap Analysis (2006-2020) (in Billions of $2002 \$$ ) 


\section{LIST OF FIGURES}

Figure $\quad$ Page

Figure 1.1 Nature of Disbursements by Local Governments 3

Figure 1.2 Study Framework 5

Figure 2.1 2006-2020 LRP Projects by Percentage of Total Cost

Figure 2.2 Resurfacing Improvement Hierarchy in HERS 28

Figure 2.3 HERS-ST Pavement Specification Defaults for a Selected Scenarios 29

Figure 2.4 Fifteen-year Needs by Functional Classification 32

Figure 2.5 Fifteen-year Reconstruction Needs 34

Figure 2.6 Interstate and Non-Interstate Fifteen-year Reconstruction Needs 34

Figure 2.7 Fifteen-year Rural Road Resurfacing Needs by Functional Class 36

Figure 2.8 Fifteen-year Total Resurfacing Needs 36

Figure 2.9 Fifteen-year Interstate and Non-Interstate Resurfacing Needs 37

Figure 2.10 Distribution of Interstate Pavement as of Year 2001

Figure 2.11 Methodology used for Excellent, Good and Good-To-Fair Pavements 44

Figure 2.12 Distribution of Non-Interstate NHS by Pavement Condition as of Year 200145

Figure 2.13 Distribution of Non-Interstate Non-NHS by Pavement Condition as of Year 2001

Figure 2.14 Methodology for Poor Pavements 49

Figure 2.15 Estimated Fiscal Needs and Projected Disbursements (2002 dollars) 80

Figure 2.16 Local Bridge Replacement of Monetary Needs 90

Figure 2.17 Indiana Local Bridge Rehabilitation Fiscal Needs 92

Figure 3.1 State Generated Highway Revenue Sources FY 2002

Figure 3.2 Relative Contributions from Revenue Sources FY 2002

Figure 3.3 State Highway Fund Revenue Flowchart 102

Figure 3.4 Motor Vehicle Highway Account Funding Flowchart FY 2003

Figure 3.5 Gasoline Tax Distribution Flowchart FY 2003 112

Figure 3.6 Historical Trends in Highway Revenue 118

Figure 3.7 Sources of Highway Revenue for Indiana Local Agencies 120

$\begin{array}{lll}\text { Figure } 3.8 & \text { Indiana Transportation Funding for Local Governments } & 121\end{array}$

Figure 3.9 MVHA and HRSF Revenue Trends for State Fiscal Years 1992-2002 122

Figure 3.10 MVHA Revenue Sources for State Fiscal Years 1992-2002 123

$\begin{array}{lll}\text { Figure 3.11 Federal Aid: Federal Fiscal Years 1997-2002 } & 128\end{array}$ 
Figure 3.12 MVHA and LRSF Combined Revenue for Indiana Counties, Cities and

Towns (Logarithmic Form)

Figure 3.13 MVHA and LRSF Combined Revenue for Indiana Counties, Cities and

Towns (Linear Form)

Figure 3.14 Federal Aid Revenue Projection for Local Agencies

Figure 3.15 Overall Procedure to Compare Automobile, Truck, Bus, and Motorcycle Registration Revenues and Driver License Revenue

Figure 3.16 Overall Procedure to Compute Tractor, Trailer, and Semitrailer Registration Revenues

Figure 3.17 Overall Procedure for Highway Revenue Forecasting for Indiana 


\section{LIST OF APPENDICES}

Appendix

Page

Appendix A INDOT Contracts Unit Pavement Treatment Costs 177

$\begin{array}{lll}\text { Appendix B HERS Treatment Costs } & 179\end{array}$

Appendix C AAMEX Model Curves 180

Appendix D HERS-ST Routine Maintenance Costs 182

Appendix E HPMS Record Description $\quad 183$

$\begin{array}{lll}\text { Appendix F HERS-ST Default Values } & 185\end{array}$

Appendix G Bridge Data Items 186

Appendix H Highway Disbursement Analysis for Indiana Counties 190

Appendix I Local Bridge Sufficiency Ratings by County 197

Appendix J Cost Estimations for Local Bridge Replacement 198

Appendix K Cost Estimations for Local Bridge Rehabilitation 199

Appendix L Summary of Local Community Highway Projects 200

Appendix M Fuel Efficiency by Model Year (miles per gallon) 202 


\section{CHAPTER 1: INTRODUCTION}

\subsection{Background Information}

Highway financing in Indiana has been characterized primarily by a "pay-as-you-go" nature. This approach has served the state well for many years, but there are indications that it is not likely to keep pace with changing trends in the highway sector and consequent emergence of new perspectives of highway investment. Many state and local governments are finding themselves caught between rising costs and reluctant taxpayers (Pennington 1995). In the state of Indiana, fluctuating economic trends engender cyclical state deficits. Uncertainties of sustained funding for highways, coupled with increasing user expectations, greater emphasis on accountability of highway related expenditure (GASB 1999) and other factors have precipitated the need for state and local highway agencies to identify and evaluate alternative sources of highway finance and also to seek costeffective investment practices. Today's transportation policy makers will have to be creative in the area of highway financing as they seek out the funding mechanisms needed to maintain and improve our transportation systems.

Over the past four decades, the Highway Trust Fund, created by the Federal Aid Highway Act of 1956, has provided a stable funding source for highway investments. As such, investments on state and local highway systems have been mainly supported by federal and state fuel taxes and other related fees (FHWA 2000). In Indiana, fuel taxes, which consist of gasoline/gasohol taxes, motor carrier surtax and fuel use, and tax on special fuels such as diesel, constitute the largest and most stable source of highway revenue (Varma and Sinha 1990), contributing approximately $70 \%$ of overall revenue (INDOT 2001). Non-fuel tax revenue, which consists of license and registration fees, permits and miscellaneous sources, account for approximately $30 \%$ of overall revenue (INDOT 2001). Through the passage of the Intermodal Surface Transportation Act (ISTEA) of 1991, state and local governments were offered options for financing highway infrastructure projects. Similar subsequent legislations that followed this lead were the National Highway System Designation Act, the Transportation Infrastructure Finance and Innovation Act (TIFIA) of 1998, and the Transportation Equity Act for the 21st Century (TEA-21) of 1998 (Dornan 2001). Traditional road funding methods invested authority in the FHWA in dictating terms and conditions for administering highway investment funds. However over the last decade, such authority has gradually shifted to the state and local governments due to their increasing involvement in highway finance (Drike and Sinha 2002).

The Indiana Department of Transportation's 2000-2025 Long Range Plan calls for the implementation of hundreds of capacity expansion projects and forecasts that the construction 
program will cost several billion dollars. Meanwhile, Indiana has been experiencing serious revenue shortfalls. As of 2002, Indiana was projected to lose $\$ 3.1$ billion in revenue that it would have received had it achieved its average revenue growth (Reed 2002). Even though transportation infrastructure is supported by user fees, the condition of the overall statewide budget status is pertinently low. In a climate of state deficits and job insecurity, policy makers may not be supportive of tax increases. This is not only limited to tax increases for education, health care, or law enforcement, but also for tax increases in general which would include transportation user fees such as a fuel tax. The state of Indiana and many others are now reviewing ways to balance their budgets, deliver public services more efficiently and fund transportation infrastructure programs without having to rely on tax increase.

Road financing needs are equally critical for local governments, as identified in a recent study conducted by the Local Technical Assistance Program at Purdue University (LTAP 2001). At the local level, highway projects are primarily funded through two major funds, the Motor Vehicle Highway Account (MVHA) and the Local Road and Street Fund. Revenue derived from these two funds account approximately for $66 \%$ of overall local revenue. Supplemental funding is also obtained through different taxes such as property taxes, income taxes, and miscellaneous taxes and constitutes $16 \%$ of the total revenues. The other revenue sources for local governments are the Federal Aid and two bridge-oriented funds, the Cumulative Bridge Fund and the Major Bridge Fund. In 2001, the total revenue for local agencies attained approximately $\$ 895$ million. The biggest part of the revenue is dedicated to maintenance (including traffic services and administration) which represents $70 \%$ of the total disbursements followed by capital outlay expenditures which account for $24 \%$ of the disbursements. The other expenses are dedicated to administration, law enforcement and safety purposes, bond retirements and transfers to state governments. Figure 1.1 illustrates the nature of disbursements by local governments.

It is obvious that revenue from current sources of highway finance may not suffice if the long-range plan is to be realized. There is therefore a need to reliably assess the current and future trends in highway financing needs both at the state and local levels vis-à-vis revenue projections, identify any future gaps in financing, and to evaluate various alternative financing schemes to address such possible shortfalls. 


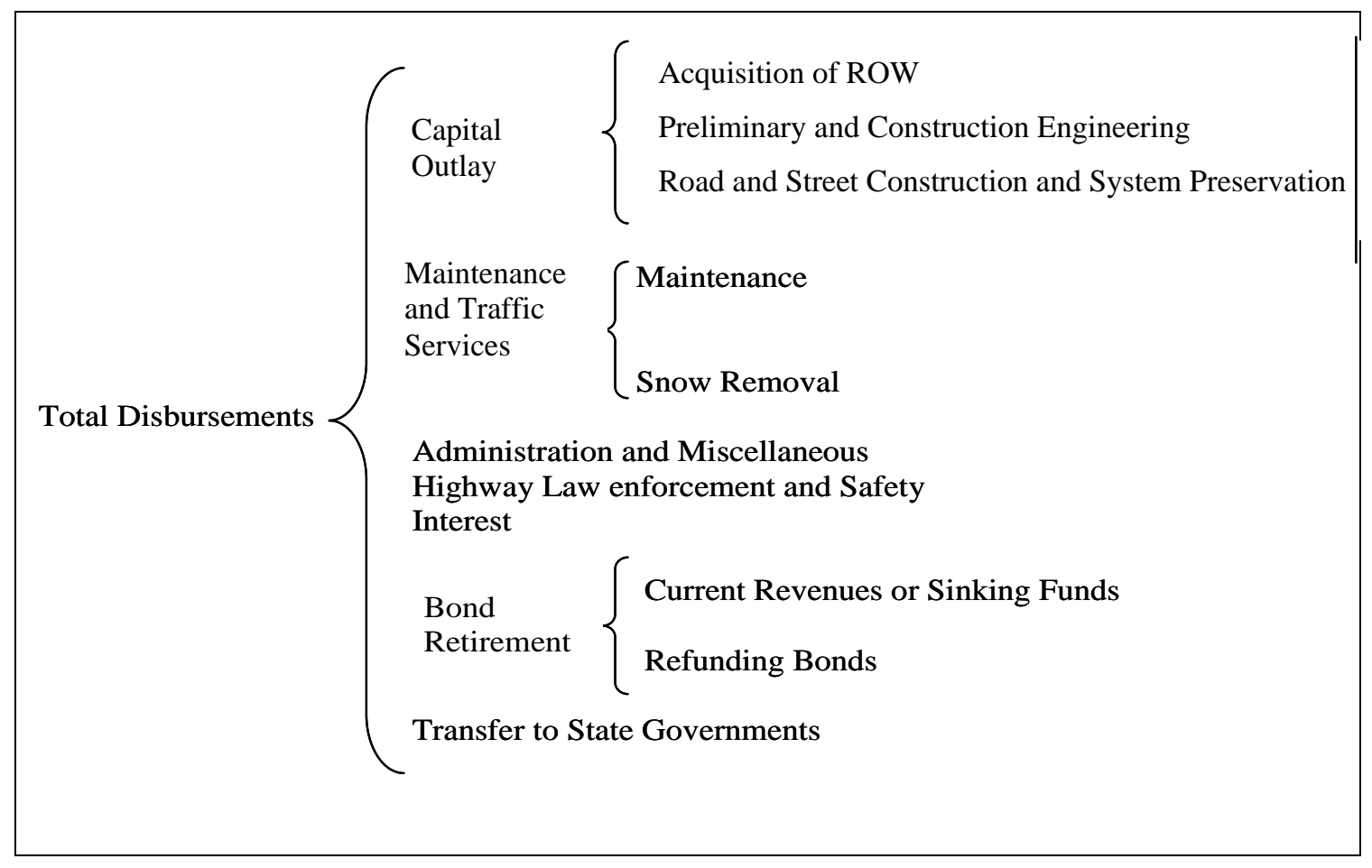

Figure 1.1: Nature of Disbursements by Local Governments

$$
\text { Source: (BTS 2000) }
$$

\subsection{Study Objectives}

On the basis of the background information and problem statement discussed in the previous section, the objectives of the study are as follows:

1. To estimate state and local highway funding needs for the 15-year period, 2006-2020.

2. To document past and current distribution of state and local highway revenue, and to make revenue predictions based on current trends and sources using a detailed forecasting methodology as well as simple projections of historical trend.

3. To identify the magnitude of any financing gap over a selected horizon period and to evaluate the feasibility of implementing various alternatives for raising additional revenue for state and local systems. 


\subsection{Scope of the Study}

The present study provides a flexible and easily accessible methodology that will help state and local agencies to track past, current and future demand (highway financing needs) as well as supply (revenue projections) under a wide range of scenarios. Using such a methodology, short-term and long-term financing plans can be developed both for state and local highway networks. The methodology is demonstrated for a 2006-2020 horizon period, using INDOT's long range plan for highways and local needs assessments, and current and innovative financing mechanisms.

\subsection{Study Framework}

To achieve the above stated objectives, the study followed a sequential approach that began with a detailed information search. This was followed by data collection, dataset preparation and data analysis. The study framework is illustrated in Figure 1.2. 
NEEDS

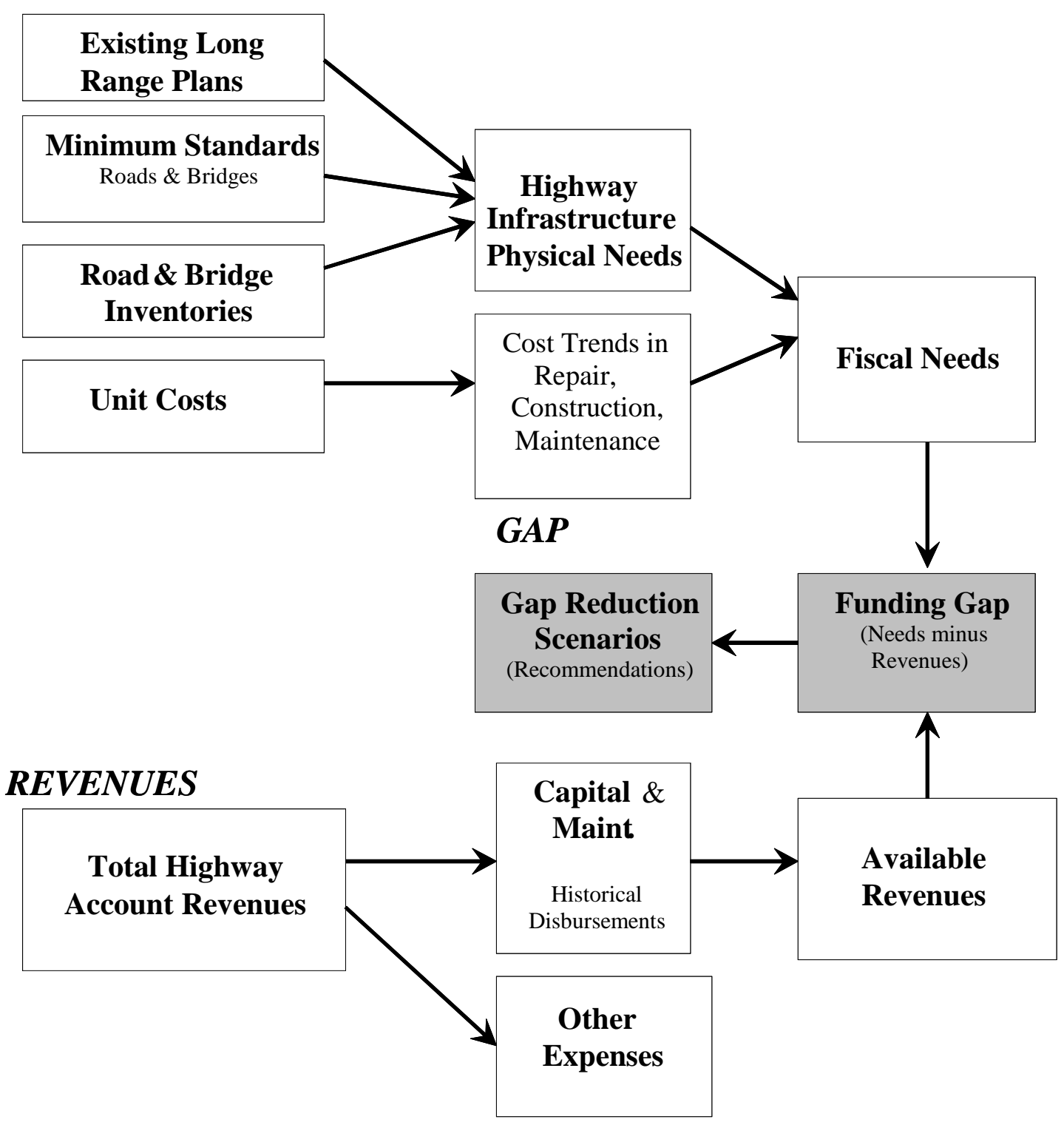

Figure 1.2: Study Framework

\subsubsection{Highway Infrastructure Needs Assessment}

Projections in funding needs for the state highway system were estimated from a variety of sources, as enumerated below:

- INDOT's 2000-2025 Long Range Plan was reviewed to obtain capital project needs at state level during the study period. 
- Data from the Pavement and Bridge Management Systems of the Program Development Division were used to determine the condition of the highway system.

- Two methods were used to estimate the pavement, safety and capacity needs. The first method was a manual network condition analysis method and the second method was the use of the FHWA Highway Economic Requirement System (HERS-ST) Model. The HERS-ST model provides an indication of the funding levels needed to sustain certain minimum levels of service on highway facilities.

- The Indiana Bridge Management System software was used to determine the bridge preservation needs.

- Historic data on costs of highway infrastructure projects and maintenance were obtained from INDOT to determine costs and spending trends.

Different sources of data were used for projecting the local needs, most of which were obtained from the Indiana Local Technical Assistance at Purdue University (LTAP), as indicated below:

- LTAP Road Condition Survey of 3,200 miles of county roads in eight separate counties; Fall 2000-Spring 2001.

- 2000 Summary of Local Road and Street Inventory Data for Indiana LPA's.

- Indiana Department of Transportation (INDOT), 2002 Highway Performance Monitoring System (HPMS) Data Base.

- National Bridge Inspection Standard Data, 2000-2001.

- County Annual Operational Reports.

A number of other sources were also used to find different maintenance and upgrading costs:

- Needs Assessment for Local Roads and Streets (LTAP 2001).

- Modeling the Cost of Bridge Replacement for Indiana County Highway Department (Elridge 2001).

- An Assessment of Preservation Needs of State Highway Bridges (Rodriguez 2004).

- Unpaved Road Maintenance Management in Local Highway Systems (Riverson 1983).

- Local Highway Agencies.

\subsubsection{Revenue Analysis}

Analysis of historical revenue levels was done to document and assess the current sources of state and local highway financing. This included the identification of the various sources of revenue, and determination of the amounts realized from each source. An analysis of expected revenue levels 
was conducted based on the historic trends in highway financing as well as by using a detailed forecasting methodology. Possible shortfalls in funding were investigated by comparing the estimated 2006-2020 needs and the projected revenues for that time horizon. A sensitivity analysis of the revenue forecast was also done and various alternative approaches were reviewed for meeting the funding gap. 


\section{CHAPTER 2: NEED ASSESSMENT}

\subsection{Estimation of Physical Needs for State Highway Network}

Recent highway needs studies in Florida and North Carolina were reviewed for methodology and lessons learned. The Florida study (CUTR 1995) originated from an interest in providing information to assist the Florida Legislature in developing potential future legislative initiatives, one of which was transportation infrastructure needs. The study described the current condition of each transportation mode in Florida, and quantified the needs for each mode for a 20 -year period from 1993-2012. The study also described the current and historical funding for transportation in Florida and other states, and forecasted revenue for the 20 -year period. The study concluded by reviewing various options and recommendations for providing sufficient revenue to meet Florida's transportation needs (CUTR 1995). At the time of the 1995 study the Florida roadway system consisted of 110,569 centerline miles and 10,856 bridges, which were separated into state and local jurisdictional responsibilities. The state had responsibility for 10.7 percent of Florida's centerline miles of road, and the local governments had jurisdictional responsibility for the remaining 89.3 percent of centerline miles. Key assumptions of the Florida Study are listed below:

- Population growth at 1.6 percent per year over the 20 year period, based on the medium growth rate forecast by the Florida Bureau of Economics and Business Research.

- Except for the road and bridge needs, inflation assumed to increase an average of 3.4 percent a year, as forecasted by Data Resources, Incorporated.

- Construction index of 3.13 percent per year over the 20-year period, for roads and bridges. This was a Florida Department of Transportation construction index.

- Right-of-way and other non-construction costs assumed a 5 percent per year increase.

- Federal revenue increase of 1 percent per year.

- State revenue increase of 4 percent per year.

- Rate of growth of vehicle miles of travel (VMT) over 20 year horizon, 3 percent per year was provided by the Florida Department of Transportation and used in the HERS analysis.

Roadway data for the Florida needs study was obtained from the Highway Performance Monitoring System (HPMS). The HPMS Analytical Process made available to the states by FHWA was used to process the HPMS data. The national default costs used in the analytical process were 
amended by an adjustment factor to represent Florida specific costs. The adjustment factor was derived using a market choice set of actual material and labor costs in Florida.

The bridge needs were estimated using the bridge data in the National Bridge Inventory (NBI). As the road data was found in HPMS data and analyzed using the FHWA Analytical Process (HERS-ST), the NBI data was processed with another FHWA process, the Bridge Needs and Investment Process, (BNIP). FHWA developed BNIP to estimate current and future bridge needs, both backlog and accruing. The process projects the deterioration of bridges and estimates the conditions for a specified analysis period.

The North Carolina Study (2000) assessed the condition of North Carolina's 79,000 mile state owned highway system and estimated the cost to repair the system and improve the deficiencies to prudent standards within an 8 year period. The study also identified revenues that would be likely available and suggested various strategies for funding the work needed. The study found that $\$ 27.8$ billion would be needed to address near-term needs, with new roads accounting for $\$ 13.8$ billion, $\$ 8.16$ billion for widening lanes, $\$ 2,4$ billion or less for pavement repairs, capacity related widening, bridge work, Interstate pavement, and shoulder widening. HERS-ST was not used to determine the road needs in the North Carolina study. The primary data sources used to assess the road needs were:

- North Carolina Department of Transportation (NCDOT) 1997/1998 pavement condition survey containing pavement condition, number of lanes, shoulder and lane width and other features for state-owned roads.

- Interstate system condition summaries from the Bureau of Transportation Statistics (BTS).

- Summaries on North Carolina's highway congestion by class of road from BTS.

- North Carolina's 1998 bridge inventory with bridge condition information.

- North Carolina Highway Design Manual, for standards for pavement work, lane widths, shoulder widths, and other criteria.

- Costs of work were obtained from preliminary work figures that NCDOT uses for planning and preliminary design.

- Typical NCDOT pavement treatments for major highways in various condition levels were summarized for cost calculations. For example, a typical pavement treatment for Interstates in fair condition was a $2-1 / 2$ inch asphalt overlay while a pavement in poor condition would be assigned a 3-inch overlay.

- NCDOT typical purchase costs for asphalt were obtained. They ranged from $\$ 3.5$ to $\$ 6.00$ / square yard with the higher cost for the thicker treatments. 
- Mobilization added $35 \%$ to cost estimates per NCDOT.

- Engineering costs added $15 \%$ to cost estimates per NCDOT.

The interstate pavement repair needs were calculated using a spreadsheet that associate the number miles within specific ranges of IRI and a specific pavement repair and unit cost were applied to those miles. North Carolina has 100 counties. The paved road system is rated by county engineers every 2 years, and the data is available in the NCDOT Pavement Survey. The roads are rated on a scale of 0 to 100 , but NCDOT does not have descriptive words for the various levels of condition. However, it is generally agreed that a score of 49 or less is poor and roads rate 29 or less are very poor. This NCDOT pavement survey was used to determine the non-interstate pavement in needs. Other need estimates included lane widening and shoulder widening needs. New road costs were determined from the Transportation Improvement Plan, a biennial document identifying all repair and new facility needs for a 6 year period.

North Carolina has about 21,241 bridges, one of the largest inventories in the United States. There are 17,035 state bridges, 34.9 percent of which were rated as deficient. The bridge needs were determined using the National Bridge Inventory and a typical NCDOT costs for repair of $\$ 25 /$ square foot, plus mobilization, engineering and inflation. The study found that $\$ 1.124$ billion dollars would be needed in the 8 year period to address importation bridge needs.

\subsubsection{INDOT 2000-2025 Long Range Plan Projects}

In the development of the highway infrastructure needs, it was necessary to review the LRP to identify the planned highway improvements for the State of Indiana. The LRP was reviewed to find projects that fell within the time horizon for the present study. Three hundred fifty-five projects were listed between the year 2006 and 2020. Typical expansion projects require a minimum of seven to eight years to develop the four stages of planning and environmental studies, design engineering, land acquisition, and construction. A full description of the added capacity project types as identified in the INDOT LRP follows:

1. Added Travel Lanes - Construction of additional travel or through lanes to existing roadways for increased capacity to obtain a more efficient and safer facility. The existing pavement is typically reconstructed at the same time.

2. New Road Construction - Construction of a new or relocated roadway, partially or fully on a new alignment.

3. Reconstruction - Projects that resurface, restore, rehabilitate, and reconstruct the existing pavement (4R) and provide some traffic flow and operational improvements via wider travel lanes, wider shoulders, sight distance improvements, and horizontal/vertical curve corrections and which are included as capacity projects. 
4. Rehabilitation - Projects that resurface, restore, and rehabilitate the existing pavement (3R) and provide some traffic flow and operational improvements via wider travel lanes, wider shoulders, sight distance improvements, and horizontal/vertical curve corrections and which are included as expansion projects but are funded from preservation program funding rather than expansion program funding.

5. TSM - Transportation System Management (TSM) is a placeholder identified in built-up urban areas experiencing capacity problems having limited right-of-way that essentially prevents adding travel lanes. The improvement options are identified after further studies are performed at the location. Possible improvements include operational improvements, one-way road pairs, intersection improvements, turn lanes, bypass and access control.

6. Median Construction - Provision of a continuous two-way left turn lane in the center of the roadway to improve the safety and capacity of a roadway, generally by reconstructing the existing pavement.

7. Interchange Modification - Improvements to an interchange, ranging from ramp terminal improvements, eliminating two-way ramps, or adding lanes to ramps to replace existing movements with loop ramps or directional ramps.

8. New Interchange Construction - Construction of a new interchange as an improvement to an existing roadway, generally to decrease congestion and improve safety.

9. Interchange - A placeholder for future interchange improvements.

10. New Bridge Construction - Construction of a major new bridge structure or a grade separation where one did not exist before which results in increased capacity and safety.

11. Freeway Upgrade - Construction of new interchanges and grade separations and reconstructing existing pavement (and possibly, added travel lanes) to improve the traffic carrying capacity and safety of an existing roadway by eliminating all at-grade intersections and railroad crossings and fully limiting access to and from the highway at interchanges only.

A gray area identified in the INDOT LRP is the improvement of an existing two-lane road or the construction of a new two-lane road that significantly upgrades the carrying capacity of the roadway. For many of these types of improvements the roadway is improved enough to be considered an expansion project. These projects typically include providing wider lanes, wider shoulders, straightening curves, leveling vertical curves and sag curves, and better controlling adjacent property access points, such as driveways, to allow for the improved flow of traffic.

The capital improvement projects identified in the INDOT 2000-2025 LRP are discussed in this section. INDOT's Program Development Division provided a database containing all of the 
LRP projects for use in the present study. The database contained a total of 530 projects. The database was sorted to reduce the universe of projects to only those that fell within the 2006-2020 horizon. All costs in the database include right-of-way, preliminary engineering, scoping and design, and construction phases. The project costs were provided in year 2002 constant dollars.

A total of 311 projects are planned between 2006 and 2020 with a total cost of $\$ 9,760,285,000$ (2002\$), a summary of the categories of projects is shown in Table 2.1. The planned projects cover nine categories of work: Added Travel Lane, Reconstruction, TSM, Interchange Study, Interchange Modification, New Interchange Construction, New Road, New Bridge, and Median Construction.

The reconstruction projects included in the list of projects provide some traffic flow and operational improvements, but there are additional reconstruction projects that are not included because they are limited to reconstructing existing pavement with no capacity-related improvements. Added travel lanes represent the largest share of the planned work, representing $48.5 \%$ of the total cost. The second largest share of planned projects is new roads, with $29.1 \%$ of the total cost. Figure 2.1 shows a summary of the planned work as a percentage of the total costs.

The new I-69 project between Evansville and Indianapolis is planned between 2018 and 2023, which is part of a larger national proposal to connect the three North America trading partners of Canada, the United States, and Mexico (INDOT 2003d). This connection is planned by using an Interstate highway located in the states of Michigan, Indiana, Kentucky, Tennessee, Mississippi, Arkansas, Louisiana, and Texas. Not all I-69 projects in the LRP are part of this larger new I-69 corridor. Four I-69 projects represent parts of the new I-69 Interstate corridor project (INDOT 2004a).

Table 2.1 2006-2020 INDOT Long Range Plan Projects Summary

\begin{tabular}{ccc}
\hline Project Type & $\begin{array}{c}\text { No. of } \\
\text { Projects }\end{array}$ & $\begin{array}{c}\text { Total Cost } \\
(1000 \mathrm{~s})\end{array}$ \\
\hline Added Travel Lane & 148 & $\$ 4,676,002$ \\
Reconstruction & 20 & $\$ 263,699$ \\
TSM & 26 & $\$ 60,749$ \\
Interchange Study & 13 & $\$ 254,250$ \\
Interchange Modification & 24 & $\$ 585,206$ \\
New Interchange Construction & 29 & $\$ 509,390$ \\
New Road & 27 & $\$ 2,896,568$ \\
New Bridge & 5 & $\$ 382,336$ \\
Median Construction & 19 & $\$ 132,085$ \\
\hline Total & $\mathbf{3 1 1}$ & $\mathbf{\$ 9 , 7 6 0 , 2 8 5}$ \\
\hline
\end{tabular}

Source: INDOT 2004b 
Costs expressed in Year 2002 constant dollars.

a. Total costs calculated by Hodge (2004). Planned projects between 2006 and

2020 summarized from LRP raw data obtained from the INDOT Program

Development Division in February 2004.

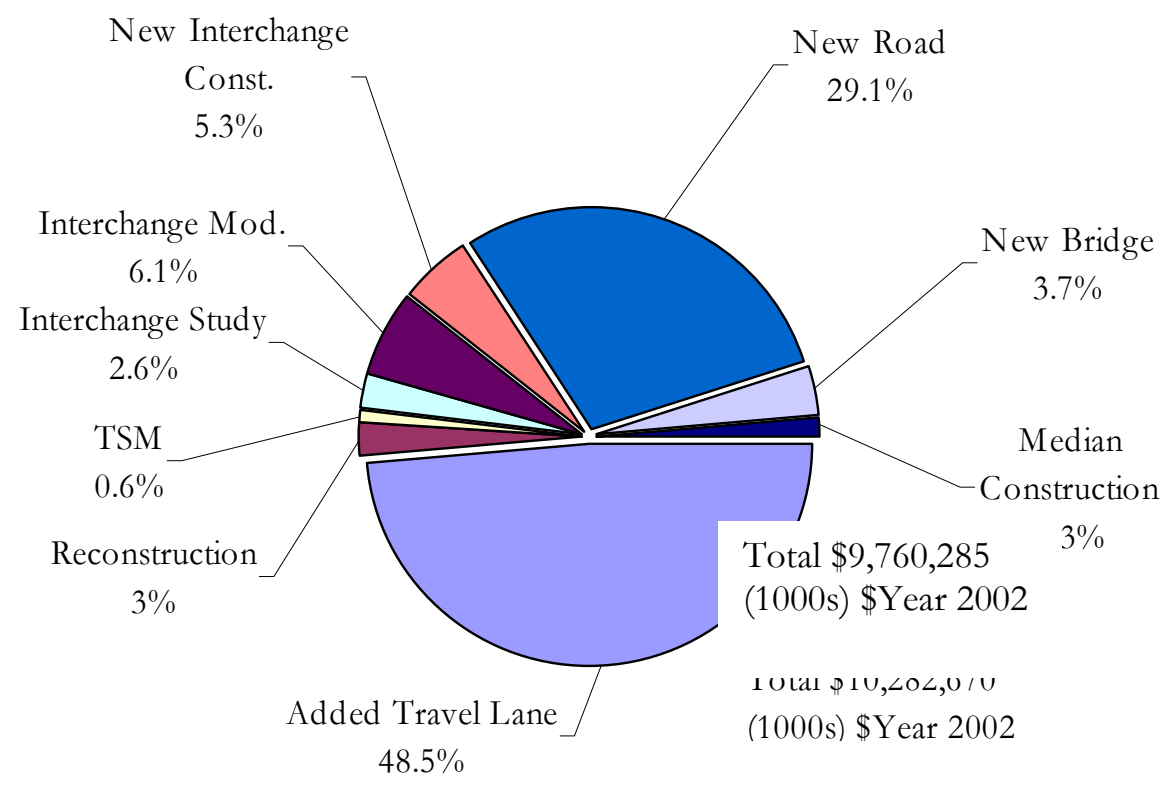

Figure 2.1 2006-2020 LRP Projects by Percentage of Total Cost (INDOT 2004b)

There are three freeway upgrade projects in the LRP with a total cost of $\$ 320$ million, which are planned for years 2021, 2023, and 2025 and outside the time horizon of this study. In addition, there are four projects listed as "Undetermined" whose timings were also outside of the time horizon used in the present study. However, due to the significant funding needs associated with such projects, they are mentioned here. The Suburban Transportation Needs project is anticipated to occur in 2028 with a planned cost of $\$ 500$ million and three Central Indiana Suburban Transportation Solution projects are planned for implementation in 2026 and 2027 with a total cost of one billion dollars. These five projects represent over 1.8 billion dollars in needs.

\subsubsection{New Bridges and Roads in Long Range Plan}

Construction of five new bridges was identified in the LRP, two to be implemented in 2006, two in 2013, and one in 2016. Descriptions of the new bridges and their costs are shown in Table 2.2. INDOT practices suggest that bridges receive a major repair such as deck rehabilitation, superstructure repair, or bridge rehabilitation every 20 or 25 years to extend the design life to 70 to 
80 years. As the first year of the planned bridge construction is 2006 , it is expected that none of the new bridges would need major repair before the end of the study period (Year 2020).

Table 2.2 Planned New Bridges 2006-2020

\begin{tabular}{clcc}
\hline Year & \multicolumn{1}{c}{ Location Description } & Route & Cost (1000s) \\
\hline 2006 & At Norfolk Southern RR, 2.11 km south of SR 3 & SR 67 & $\$ 4,730$ \\
2006 & At Centennial Ave, 1.61 km north of SR 32 & US 35 & $\$ 1,630$ \\
2013 & Extend I-265 into Kentucky (Indiana share) & I 265 & $\$ 101,376$ \\
2013 & New Ohio River Bridge & I 65 & $\$ 249,600$ \\
2016 & Over Ohio River (Indiana share) & US 421 & $\$ 25,000$ \\
\hline
\end{tabular}

Source: INDOT 2004b

Costs expressed in Year 2002 constant dollars.

A total of 1,631 lane-miles are planned to be added to the state highway system between 2006 and 2020. Forty-seven percent of the added-lanes are to be constructed on the interstate system. The total capital improvement cost for the added-lanes is $\$ 4,676,002,000$. The 15 -year pavement preservation for added-lanes is discussed in Section 2.1.7.

Twenty-seven new roads are planned between 2006 and 2020, and involve a total of approximately 1,168 lane-miles. The costs associated with new roads account for 29.1 percent of the total LRP costs in the 15-year horizon. Sixty and forty percent of the new roads are on the interstate and non-interstate systems, respectively. The yearly distribution of lane-miles of new roads between 2006 and 2020 is illustrated in Table 2.3. The new roads planned between 2006 and 2020 are described in Table 2.4. The total capital cost for planned new roads is $\$ 2,896,568$ (in Year 2002\$), which includes 700 lane-miles of new interstate and 468 lane-miles of new non-interstate pavement.

Table 2.3 Lane-Miles of Roads by Year and Category 


\begin{tabular}{cccc}
\hline $\begin{array}{c}\text { Year of } \\
\text { Implementation }\end{array}$ & $\begin{array}{c}\text { Total New Road } \\
\text { Lane-Miles }\end{array}$ & $\begin{array}{c}\text { Interstate } \\
\text { Lane-Miles }\end{array}$ & $\begin{array}{c}\text { Non-Interstate } \\
\text { Lane-Miles }\end{array}$ \\
\hline 2006 & 40.3 & 0.0 & 40.3 \\
2007 & 152.0 & 0.0 & 152.0 \\
2008 & 64.3 & 0.0 & 64.3 \\
2009 & 5.2 & 0.0 & 5.2 \\
2010 & 5.8 & 0.0 & 5.8 \\
2011 & 83.5 & 0.0 & 83.5 \\
2013 & 96.0 & 22.8 & 73.2 \\
2017 & 86.5 & 78.1 & 8.4 \\
2018 & 328.0 & 328.0 & 0.0 \\
2019 & 34.8 & 0.0 & 34.8 \\
2020 & 271.5 & 271.5 & 0.0 \\
\hline Total & 1167.8 & 700.4 & 467.8 \\
& \% of Total & $\mathbf{6 0}$ & 40 \\
\hline
\end{tabular}

Source: INDOT 2004b

Table 2.4 Planned New Roads 2006-2020

\begin{tabular}{llcc}
\hline Year & \multicolumn{1}{c}{ Location Description } & Route & $\begin{array}{c}\text { Cost }^{\mathrm{b}} \\
(1000 \mathrm{~s})\end{array}$ \\
\hline 2006 & 0.8 mile S. of SR 60 (Jackson St) E. to SR 60 (east of Salem) & SR 135 & $\$ 2,868$ \\
2006 & Western Bypass of Paoli & SR 37 & $\$ 14,080$ \\
2006 & Extension of US 12/20 to Lake Michigan (Gary Marina) & Sa & $\$ 11,230$ \\
2006 & SR 26 to US 52 (around the west side of Lafayette) & US 231 & $\$ 14,270$ \\
2006 & SR 56 (east of Salem at Quaker Rd) south to SR 60 & SR 60 & $\$ 4,895$ \\
2006 & 2nd and Mill St to 1st St in Hazleton & SR 56 & $\$ 474$ \\
2007 & US 421 to US 24/35 & SR 25 & $\$ 137,483$ \\
2007 & I-65 to US 421 & SR 25 & $\$ 82,517$ \\
2007 & I-80/90 (Toll Road) to I-94 & US 421 & $\$ 24,801$ \\
2008 & Huntingburg / Jasper Bypass (Stage 1) (2 lanes) & US 231 & $\$ 139,316$ \\
2008 & US 20 to Just South of 12th St. & SR 331 & $\$ 27,370$ \\
2008 & 0.5 mi E. of I-469 to 0.5 mi E. of Ryan/Bruick Rd includes & & \\
& interchange (Phase I) & US 24 & $\$ 16,568$ \\
2009 & 0.5 mi E. of Ryan/Bruick Rd to 0.5 mi E. of Webster Rd & US 24 & $\$ 21,923$ \\
& includes interchange (Phase II) & & \\
2010 & 0.5 mi W. of SR 101 to Indiana/Ohio State line includes & US 24 & $\$ 25,114$ \\
& SR101 interchange (Phase 4) & US 31 & $\$ 99,398$ \\
2011 & Existing US 31 south of Lakeville to US 20 & US 31 & $\$ 64,622$ \\
2011 & US 30 to existing US 31 south of Lakeville & US 24 & $\$ 22,000$ \\
2011 & From 0.5 mi E. of Webster Rd to 0.5 mi W. of SR101 (Phase & & \\
& III) & I 265 & $\$ 129,024$ \\
2013 & Extend I-265 into Kentucky (Road) & US 31 & $\$ 130,000$ \\
2013 & South of SR 26 to SR 18 & SR 267 & $\$ 4,746$ \\
2017 & SR 67 to SR 267 south of I-70 & I 69c & $\$ 200,000$ \\
2017 & Placeholder for Henderson to Evansville Study & $\$ 262,486$ \\
& $\begin{array}{l}\text { Recommendation } \\
\text { Placeholder for I-69 from SR 144 to I-465 (segment of }\end{array}$ & & \\
& independent utility) & &
\end{tabular}


2018 Placeholder for Evansville to Indianapolis (I-69)

I 69c $\$ 714,000$

2019

1.1 mile west of Daviess / Martin County Line to Daviess /

US 50

$\$ 2,651$

Martin County Line

2019 Daviess / Martin County Line to East Fork White River

US $50 \$ \$ 10,446$

East Fork White River to 0.1 mile east of US 150

US $50 \quad \$ 10,772$

2020 Placeholder for Evansville to Indianapolis (I-69 Seymour District to SR 144)

I 69c $\$ 723,514$

a. A route number was not provided in source data.

Total $\mathbf{\$ 2 , 8 9 6 , 5 6 8}$

b. Costs expressed in Year 2002 constant dollars. INDOT 2004b is the source of the raw data. Calculations done in present study to summarize projects by date.

c. Part of the New I-69 Corridor.

\subsubsection{Pavement Condition and Design Life}

Pavements are typically designed for 15 to 30-year design lives (INDOT 1998). Table 2.5 presents the typical design life of various pavement treatments as provided by INDOT.

\section{Pavement Condition}

Pavement condition influences user costs, such as vehicle operating costs, safety, and travel time. Two measures of pavement condition were used in this research, the Pavement Serviceability Rating (PSR) and the International Roughness Index (IRI). The Pavement Serviceability Rating (PSR) is a subjective rating of pavement ride quality which requires visual inspection of the pavement. According to the INDOT Design Manual (IDM) Chapter 52, the pavement is rated from 0 to 5 , where 0 is totally impassable or failed pavement and 5 is a pavement in excellent condition. The IDM assumes an initial serviceability index of 4.2 .

Table 2.5 Design Life of Pavement Treatments 


\begin{tabular}{lc}
\hline \multicolumn{1}{c}{ Pavement Treatment } & Design Life (years) \\
\hline New PCCP & 30 \\
Concrete Pavement over Existing Pavement & 25 \\
New Full Depth HMA & 20 \\
HMA Overlay over Rubblized PCCP & 20 \\
HMA Overlay over Asphalt Pavement & 15 \\
HMA Overlay over Cracked and Seated PCCP & 15 \\
HMA Overlay over CRC Pavement & 15 \\
HMA Overlay over Jointed Concrete, Sawed and Sealed Joints & 15 \\
HMA Overlay over Jointed Concrete & 12 \\
PCCP Joint Sealing & 8 \\
Thin Mill and Resurface of Existing Asphalt & 8 \\
Concrete Pavement Rehabilitation (CPR) Techniques & 7 \\
Microsurface Overlay & 6 \\
Chip Seal & 4 \\
Asphalt Crack Sealing & 3 \\
\hline
\end{tabular}

Source: Indiana Design Manual, Chapter 52, 1998

IRI is a physical measure of the pavement ride quality and captures the "bumpiness" of the pavement in terms of inches per mile. The higher the IRI value, the rougher is the ride. A review of available data, by Lamptey et al. (2004), suggests that a new flexible pavement would have an initial IRI of 60 and typical new rigid pavement would have an initial IRI of 70 . A summary of the IRI index, as provided by the Pavement Management Section of the Program Development Division of INDOT, is illustrated in Table 2.6.

Table 2.6 International Roughness Index

\begin{tabular}{cc}
\hline Pavement Condition & IRI Range \\
\hline Excellent & $60-100$ \\
Good & $100-150$ \\
Fair & $150-200$ \\
Poor & $>200$ \\
\hline Source: INDOT 2000
\end{tabular}

Over time, new pavements deteriorate due to traffic loads and weather effects, and the PSR value decreases. A pavement is considered to have reached its terminal serviceability between a PSR 
of 2.5 to 2.0 , depending on its functional classification. A summary of terminal serviceability ratings for pavements is shown in Table 2.7 .

Table 2.7 Terminal Pavement Serviceability Ratings

\begin{tabular}{lc}
\hline \multicolumn{1}{c}{ Pavement Classification } & PSR \\
\hline Rural major collector and above & 2.5 \\
Rural minor collector and below & 2.0 \\
Urban arterials & 2.5 \\
Urban collectors and below & 2.0 \\
\hline Source: INDOT Design Manual, Chapter 52, 1998 &
\end{tabular}

\section{Pavement Deterioration Rates}

Pavement deterioration curves developed by Lamptey et al. (2004) suggest that the average rate of deterioration for Indiana pavements can be taken as 0.2 PSR per year. To determine the corresponding change in IRI associated with a $0.2 \mathrm{PSR} /$ year deterioration rate, the INDOT equation 2.1 (Gulen et al. 1994 and INDOT 2000) relating IRI to PSR was used.

$$
P S R=9.0 \times \varepsilon^{(-0.008747 \times I R I)} \quad \text { Eq. } 2.1
$$

According to Eq. 2.1, a new pavement with an initial PSR of 4.2 and a pavement deterioration rate of $0.2 \mathrm{PSR} /$ year has an equivalent change in IRI due to pavement deterioration of 6.0 IRI/year. The pavement needs analysis for the manual network condition method is based on two levels of deterioration rates of $0.2 \mathrm{PSR} /$ year and $0.3 \mathrm{PSR} /$ year, which correspond to deterioration rates of $6.0 \mathrm{IRI} /$ year and $8.0 \mathrm{IRI} /$ year respectively, using a PSR of 4.2 as the starting condition. Table 2.8 illustrates the pavement deterioration rates in terms of IRI and PSR.

Table 2.8 Pavement Deterioration Rates

\begin{tabular}{ccccc}
\hline Initial & \multicolumn{2}{c}{ Deterioration Rate } & \multicolumn{2}{c}{ Condition After 1 Year } \\
\cline { 2 - 5 } Condition & $\Delta$ PSR/Year & $\Delta$ IRI/Year & PSR & IRI \\
Rating (PSR) & & 6 & 4.00 & 93 \\
& 0.2 & 7 & 3.95 & 94 \\
4.2 PSR & 0.25 & 8 & 3.90 & 96 \\
& 0.3 & 11 & 3.80 & 99 \\
\hline
\end{tabular}

\subsubsection{Pavement Treatments}


There are many types of treatments that can be selected to improve the condition of pavements as illustrated in Pavement Condition. Descriptions of common pavement treatments as outlined in the IDM and the April 2003 INDOT Memorandum entitled "FY-2004 Pavement Preservation Guidance (Draft)," are discussed in the sections that follow. The terms " $3 \mathrm{R}$ " and "4R," when used in a pavement treatment context, imply the following:

- $\quad 3 \mathrm{R}$ projects are used for rehabilitating the pavement. This is major pavement work that will include pavement rehabilitation or reconstruction; shoulder work such as patching and/or replacement; and limited pipe work and safety work. Work may include curb or sidewalk work and minor realignment of the road centerline at specific spot locations. No right-of-way acquisition is needed (INDOT, 2003e).

- $4 \mathrm{R}$ projects are intended to replace the entire pavement structure. This is major pavement work that generally requires the correction of all safety defects and reconstruction of items outside the pavement structure. Work includes bringing the road up to current geometric standards, upgrading all safety features, and upgrading all drainage features. Work may include added travel lanes if authorized by the INDOT LRP.

\subsubsection{Preventive Maintenance Treatments}

NCHRP Report 223 provides two convenient criteria for maintenance activities: urgency of the activity and the effect of the activity. Geoffroy (1996) provided the following descriptions for maintenance activities:

- Routine Maintenance: Day-to-day activities that are scheduled and whose timing is within the control of maintenance personnel, such as moving and ditch cleaning. "Routine maintenance" is a broad term often used to describe any activity that is carried out on a routine basis, such as routine preventive maintenance, i.e., crack sealing; routine corrective maintenance, i.e., patching; and non-pavement routine maintenance, i.e., mowing and underdrain maintenance.

- Demand Maintenance: Urgent activities that must be done in response to an event beyond the control of maintenance personnel, i.e., any emergency repair of a pavement.

- Corrective Maintenance: Planned activities to repair deficiencies, i.e., shallow patching to increase the structural capacity at a localized area. 
- Preventive Maintenance: Planned activities that correct minor defects, slow down future deterioration, and maintain and improve the functional condition of the system while not substantially increasing the structural capacity.

Preventive Maintenance (PM) is intended to extend the life of the pavement by arresting light deterioration, retarding progressive damage, and reducing the need for routine maintenance. The proper time for PM is before the pavement experiences severe distress, structural problems, and moisture or aging-related damage.

The commonly used PM treatments on asphalt surfaces include: chip sealing, crack sealing, micro-surfacing, sand sealing, and thin hot-mix asphalt (HMA) overlays with or without milling. Thin HMA overlays may involve a single course of $40 \mathrm{~mm}$ HMA. For concrete pavements, the pavement could receive Concrete Pavement Rehabilitation (CPR) techniques, such as joint sealant replacement, contract crack sealing, minor patching, and retrofit joint load transfer. Cleaning and sealing of joints for PCC pavement includes inspecting contraction and longitudinal joints for loose, missing, or depressed sealant. Defective sealants are removed and replaced. This prevents dirt and moisture from entering the joints.

\subsubsection{Pavement Rehabilitation Partial 3-R}

This treatment includes a new surface placed on the existing road to improve service. The project is not constructed to the current $3 \mathrm{R} / 4 \mathrm{R}$ standards (which could include alignment work). The primary intent is to restore the surface of the road by several methods. Incidental work such as curbs, drains, shoulders, guardrail or other facility improvements also may be included. This type of work does not widen, modernize, or significantly upgrade the facility and this work is typically funded by the State.

\subsubsection{Pavement Replacement}

This treatment replaces existing mainline pavement with new pavement. The new pavement may be wider than the existing or have a different number of lanes from the original. Incidental work such as grading, drains, shoulders, guard rail for the purpose of modernizing the facility and enhancing safety may be included. This work is typically funded by the federal government. This treatment replaces existing mainline pavement with new pavement. The new pavement may be wider than the existing or have a number of lanes that is different from the original. Incidental work, such as grading, drains, shoulders, or guard rails, for the purpose of modernizing the facility and enhancing safety may be included. This work is typically funded by the federal government. 


\subsubsection{Cost of Highway Repairs}

Several sources were investigated for Indiana-specific pavement treatment costs. Average costs per lane-mile for treatments by pavement type were obtained from the JTRP project entitled, "Life Cycle Cost Analysis for Pavement Design Procedures" (Lamptey et al. 2004) which utilized pavement construction and rehabilitation cost data from INDOT's Contracts Division. The focus of that research work was related to treatments to the traveled way; therefore, costs related to shoulder work were not included. A list of common pavement treatments and their costs per lane-mile based on the research of Lamptey et al. (2004) is provided in Table 3.9. A complete description of all of the pavement treatment costs is shown in Table A.1 in Appendix A.

Table 2.9 Average Pavement Treatment Costs per Lane-Mile

\begin{tabular}{lccc}
\hline \multicolumn{1}{c}{ Treatment } & $\begin{array}{c}\text { Flexible } \\
\text { Pavement } \\
(\text { HMA })\end{array}$ & $\begin{array}{c}\text { Rigid } \\
\text { (PCC) }\end{array}$ & $\begin{array}{c}\text { Composite } \\
(\text { COMP) }\end{array}$ \\
\hline Joint and Crack Sealing & - & $\$ 539^{\mathrm{a}}$ & - \\
Preventive Maintenance & $\$ 72,689^{\mathrm{b}}$ & - & $\$ 72,689^{\mathrm{b}}$ \\
Resurfacing Partial 3-R Standards & $\$ 297,263^{\mathrm{b}}$ & $\$ 297,263^{\mathrm{c}}$ & $\$ 297,263^{\mathrm{b}}$ \\
Reconstruction/Replacement & $\$ 1,394,329^{\mathrm{b}}$ & $\$ 1,454,117^{\mathrm{b}}$ & $\$ 1,394,329^{\mathrm{b}}$ \\
\hline Costs are expressed in Year 2002 constant dollars. & & \\
a. Labi and Sinha (2003). & & \\
b. Lamptey et al. (2004). & & \\
c. PCC pavement is assumed to be resurfaced with HMA. & &
\end{tabular}

\subsubsection{HERS-ST Analysis Treatment Costs}

All cost data used by the HERS-ST program is provided in the IMPRCOST.DAT and PARAMS.DAT files that are used to control program operation. These files include unit costs for highway improvements, vehicle operation, travel time, injuries, and property damage, as well as fuel excise taxes and the value of life. The costs are supplied in the dollars of the various years and converted to 2000 dollars using price index values contained in the PARAMS.DAT file. Indianaspecific costs were developed in 1998 by Cambridge Systematics, Inc. for INDOT's use in the HERS-IN software. These Indiana-specific cost data were obtained from INDOT for use in the present study and the default IMPRCOST.DAT and PARAMS.DAT files were adjusted based on this data. The Indiana-specific costs adjusted to $2002 \$$ are illustrated in Table 2.10. The noninflation adjusted costs in their original 1997 dollar year format can be found in Table B.1 located in Appendix B. 
The costs for HERS-ST improvements are differentiated by the following criteria: rural or urban locations, flat or rolling terrain, and the functional classification of the facility. It may be more costly to build a highway in rolling terrain because vertical alignment issues may be present; requiring cuts and fills in the soil that adds costs to the projects. It is also expected that work on an urban expressway may be more costly than work on a rural major collector because the cost of traffic control and right-of-way are typically higher in urban areas. Also, the lane widths provided on an expressway may be wider than those provided on a major collector, thereby requiring more pavement area. Additionally, a thicker pavement may be needed on an expressway carrying interstate commercial truck traffic, adding to the cost of the project. The unit costs illustrated in Table 2.10 support these assumptions as costs increase with higher functional class, urban location, and rolling terrain.

Table 2.10 Indiana-specific Costs Developed for HERS-IN

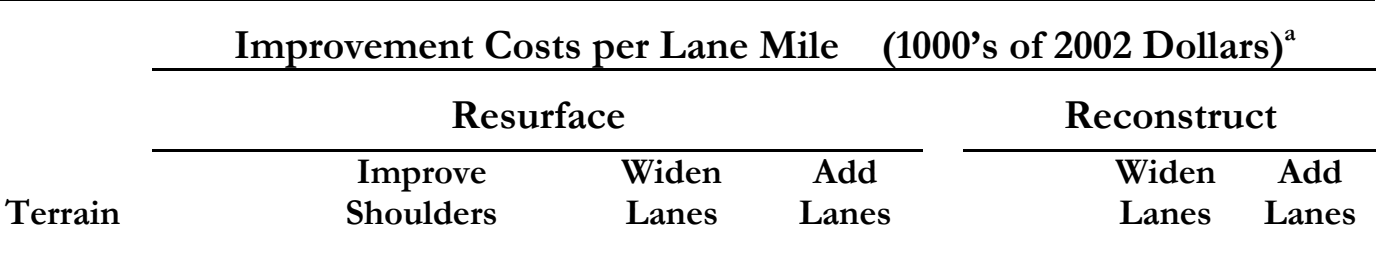




\section{Rural}

Interstate

$\begin{array}{llllllll}\text { Flat } & 102 & 212^{\mathrm{b}} & 448 & 605 & 642^{\mathrm{b}} & 729 & 1105 \\ \text { Rolling } & 106 & 231 & 486 & 655 & 706 & 795 & 1205\end{array}$

Other

Principal

Arterial

$\begin{array}{ll}\text { Flat } & 72 \\ \text { Rolling } & 77\end{array}$

153

323

436

462

525

796

Rolling 77

166

350

472

$508 \quad 572$

867

Minor

Arterial

Flat $\quad 66$

66
70

137

149

289

316

392

426

$\begin{array}{lll}417 & 472 & 717\end{array}$

Rolling

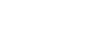

Major

Collecto

$\begin{array}{ll}\text { Flat } & 59 \\ \text { Rolling } & 62\end{array}$

$\begin{array}{ll}59 & 126 \\ 62 & 135\end{array}$

262

284

354

384

375

35

284

413

$465 \quad 706$

\section{Urban}

Freeway/

Expressway

Other

Divided

Undivided

$\begin{array}{lll}137 & 311^{\mathrm{b}} & 666 \\ 100 & 228 & 479 \\ 89 & 205 & 43 \\ & & \\ & \end{array}$

a. Excludes costs of alignment improvements.

b. Derived directly from INDOT costs.

c. These costs are per added lane-mile; all other costs are per lane-mile.

Table 2.10 Indiana-specific Costs Developed for HERS-IN (Continued)

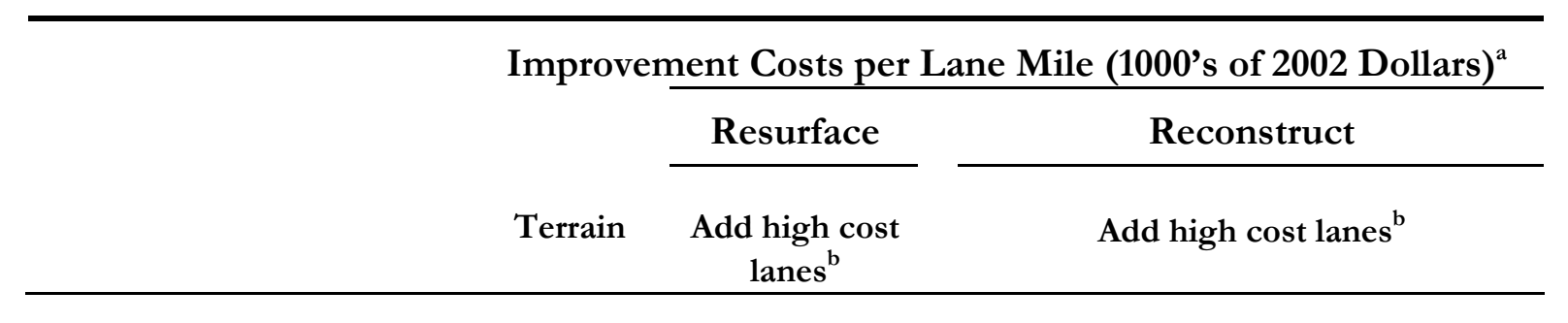




\section{Rural}

Interstate

Flat $\quad 1534$

2413

Rolling

Other Principal Arterial

Flat

1079

1055

Rolling

1127

1280

Minor Arterial

Flat

883

950

Rolling

695

1152

Major Collector

Flat

658

1068

Rolling

868

1040

\section{Urban}

Freeway/ Expressway

$4019 c$

3423

Other Divided

$2518^{c}$

2653

Undivided

a. Excludes costs of alignment improvements.

b. Derived cost based on ratio between national normal cost and national high cost lanes.

c. These costs are per added lane-mile; all other costs are per lane-mile.

The internal Parameter Model in HERS converts the 1997 dollar improvement costs to year 2000 dollars by using FHWA's composite price index for federal aid-highway construction. These HERS-ST costs were found to be comparable to the costs from the JTRP project, "Life Cycle Cost Analysis for Pavement Design Procedures" by Lamptey et al. (2004).

\subsubsection{Routine Maintenance Costs}

Average maintenance costs were obtained from models developed by Labi and Sinha (2003) for interstate and non-interstate pavements as functions of pavement age, functional class, surface type, and other pavement attributes. The models include all categories of maintenance. The expenditures were reported in 1995 dollars per lane-mile and the average values are listed here:

- Interstate PCC $-\$ 1,093$

- $\quad$ Interstate HMA $-\$ 1,100$

- $\quad$ Non-Interstate HMA - $\$ 500$

- Interstates COMP - $\$ 410$

- $\quad$ Non-Interstate COMP - $\$ 590$ 
Where two curves are provided for one road classification, the higher cost curve was used. The average costs reflect the average of all pavement ages. The maintenance expenditure models are illustrated in Appendix C. The values were adjusted to year 2002 dollars and are listed in Table 2.11.

Table 2.11 Average Annual Maintenance Costs per Lane-Mile

\begin{tabular}{cccc}
\hline Facility Type & $\begin{array}{c}\text { Flexible Pavement } \\
(\text { HMA })\end{array}$ & $\begin{array}{c}\text { Rigid } \\
(\text { PCC })\end{array}$ & $\begin{array}{c}\text { Composite } \\
(\text { COMP) }\end{array}$ \\
\hline Interstate & $\$ 1,335$ & $\$ 1,326$ & $\$ 497$ \\
Non Interstate & $\$ 607$ & $\$ 1,326$ & $\$ 716$ \\
\hline
\end{tabular}

Source: Labi and Sinha (2003).

Costs are expressed in Year 2002 dollars.

The average annual maintenance costs used by the HERS-ST software were developed by Witczak and Rada (1984). Maintenance costs were estimated in dollars per lane-mile as a function of PSI rather than age. Unit costs are based on parameters associated with the amount of damage to the pavement at each PSI level and the unit cost of repair for crack sealing, surface patching and deep patching. The Witczak and Rada routine maintenance unit costs, provided in Appendix D, do not include rut depth and roughness (IRI) corrections as they assume these issues to be corrected during major maintenance work such as overlays.

\subsubsection{HERS-ST Physical Needs Analysis}

The HERS-ST estimated needs for pavement, capacity, and safety improvements between 2006 and 2020 are discussed in this section. HERS-ST determines the needs of the existing infrastructure but does not estimate the needs for new roads and bridges. Costs for new roads, interchanges, bridges, and medians, and intersection improvements were obtained from INDOT's LRP and added to the total needs calculated by HERS-ST. This section describes the data used for the analysis and the procedure for modifying the model to be Indiana-specific with respect to the unit costs, deterioration rates, and deficiency levels used. Finally, this section outlines the results of the various scenarios that were analyzed. The HERS-ST analysis has the following characteristics:

- Only pavements and shoulders are considered;

- The analysis is aggregate in nature, providing recommended investment levels by type of improvement and functional class, but not by individual project;

- No interdependencies among highway sections are addressed in the model;

- New construction on new alignment is not included; and 
- Initial improvement costs include typical capital expenditures but the cost of delay associated with implementing improvement options is not considered.

\subsubsection{Collection of Data for HERS-ST}

The starting point for running the HERS-ST model is a HPMS data file that describes part or all sections of the State Highway System at some base year. The HPMS file contains ASCII descriptions of each highway section to be analyzed, one record per highway section. Each record contains 98 fields, such as functional class, surface type, lane width, and AADT. The full listing of the HPMS record contents is provided in Table E.1 and Table E.2 in Appendix E. The variable names shown in the tables are internal to the HERS-ST. For this study, the 2002 HPMS data set was obtained from the FHWA Office of Asset Management (FHWA 2004b) with additional information from INDOT's Program Development Division. HPMS data items 1-46 are universe data (basic inventory information required to be reported for all open-to-traffic, public road systems). Examples of universe data are: measured pavement roughness (IRI), AADT, section length, and governmental ownership (jurisdiction).

\section{Validation of Indiana 2002 HPMS Data}

The database contained 6,779 sections of highway data that included all highway jurisdictions. For the state highway system, the HPMS dataset was sorted to include only those sample sections under state jurisdiction, thus reducing the HPMS file to 1,183 sections. Further data sorting revealed that the dataset did not have sample data on state-controlled roads for two rural counties: Pike County, and White County. These two counties therefore had no data for HPMS items 47 to 98. However, the HPMS dataset does not require a specific amount of sampling per county. The highway sampling is by "Rural" and "Urban" areas rather than by county. Each HPMS sample section represents a larger number of actual highway sections, with the use of expansion factors that depend upon section characteristics such as the AADT volume group assigned by the HPMS and the functional class. Expansion factors are determined by each state. For the present study, the expansion factors for the 2002 HPMS sample of state-controlled sections were provided by the FHWA Office of Asset Management. The total mileage represented by any given section can be obtained by multiplying the length of the section by its expansion factor. The sample data for the present study was expanded to represent the universe of state jurisdictional roadway miles by applying the expansion factors provided. When expanded, the sample sections on the state highway system represent 11,120 miles or 28,310 lane-miles of roadway. This is reasonably consistent with 
the mileage reported in the 2003 INDOT Annual Report. It therefore seems reasonable to assume that the highway samples for other rural counties adequately represent those at Pike and White Counties. HERS estimates of justifiable capital expenditures are obtained by analyzing individual sample sections and multiplying the results by the section's expansion factor.

\subsubsection{HERS-ST Methodology}

HERS-ST starts the "design" process by searching for conditions that indicate deficiencies. The term "design" is used in the context of identifying deficiencies and generating improvement candidates. HERS considers present conditions and forecasts future conditions in searching for potential deficiencies. The HERS model consists of two programs; the PreProcessor and the main program. The PreProcessor requires the following input: tables containing design standards; deficiency levels for highway sections by functional system; a set of run specifications; and section data (FHWA 2001d). In basic runs, HERS-ST performs its own evaluations of the estimated costs and benefits of all potential pavements, widening, and alignment improvements and determines which improvements best meet the criteria provided by the user. Information on the highway system in the base year and forecasted traffic volumes for some specified future year are provided in the HPMS file. Estimates of justifiable capital expenditures are obtained in HERS-ST by analyzing individual sample sections and multiplying the results by the section's expansion factor.

\section{Improvement Types}

The highway improvements considered by HERS-ST consist of resurfacing or pavement reconstruction. These two options can be combined with shoulder work, widening and/or alignment improvement. The specific HERS-ST codes associated with the various treatment types are listed in Table 2.12.

Table 2.12 Codes for "HERS-Type" Improvements 
IMPRCOST

Code
Without With

Alignment Alignment Improvement Improvement

\begin{tabular}{llll} 
Rs & Resurface & 1 & 11 \\
RsSh & Resurface and improve shoulders & 2 & 12 \\
$\begin{array}{l}\text { MinW or } \\
\text { RSWL } \\
\text { RSNC or }\end{array}$ & Resurface and widen lanes (minor widening) & 3 & 13 \\
$\begin{array}{l}\text { MWNC } \\
\text { RSHC or }\end{array}$ & Resurface and add normal-cost lanes (major widening) & 4 & 14 \\
MWHC & Resurface and add high-cost lanes & 5 & 15 \\
RC & Pavement reconstruction & 6 & 16 \\
RCWL & Pavement reconstruction with wider lanes & 7 & 17 \\
RCNC & Pavement reconstruction and add normal-cost lanes & 8 & 18 \\
RCHC & Pavement reconstruction and add high-cost lanes & 9 & 19 \\
\hline
\end{tabular}

Source: Cambridge Systematics, Inc. 2004

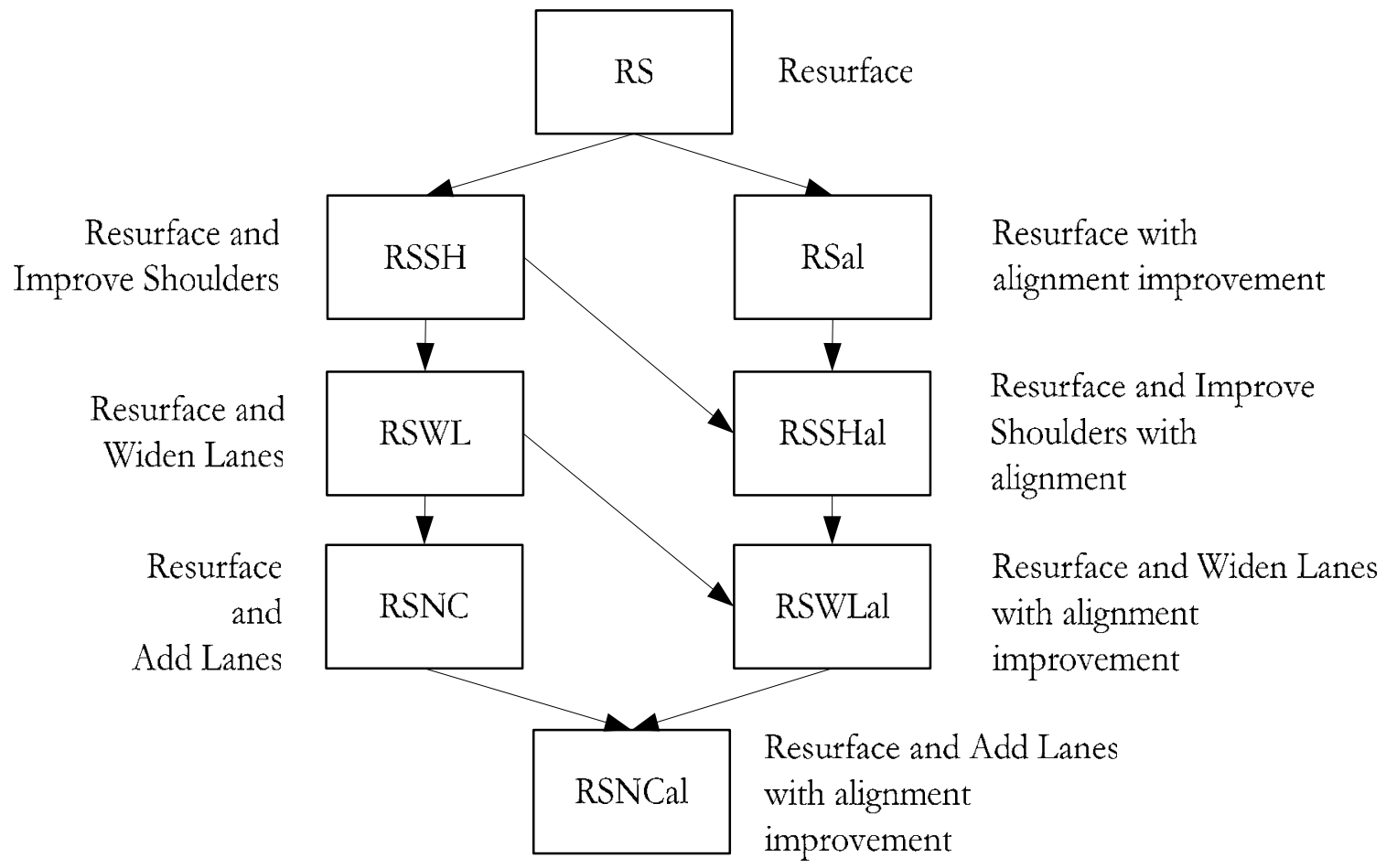

Figure 2.2 Resurfacing Improvement Hierarchy in HERS (FHWA 2001d)

For resurfacing improvement options, the hierarchy of activities is shown in Figure 2.2. Options that include alignment improvement are shown on the right side of the figure; 
improvements that include lane additions are shown on the left side of the figure. The addition of lanes is a more aggressive improvement than widening lanes, which is more aggressive than widening shoulders. If lanes are added, it is assumed that shoulder deficiencies and lane width deficiencies are corrected, and all lanes are resurfaced. HERS assumes that resurfacing is always performed using a flexible overlay. For flexible overlays over all pavement types, the overlay thickness used by HERS varies with the traffic load such as it does for reconstruction with flexible pavement. These thicknesses are shown in Table F.1 in Appendix F.

\section{Deficiency Criteria and Design Standards}

According to the HERS-ST Overview Report (FHWA 2001d), HERS incorporates several methods for identifying deficiencies and specifying improvements:

- Deficiency Levels (DL)- The basic set of deficiency criteria associated with pavement condition, surface type, volume to capacity ratio, lane width, right shoulder width, shoulder type, horizontal and vertical alignment;

- Serious Deficiency Levels (SDL) - Deficiencies that must be corrected if any improvement is made to the section, but SDLs will not be corrected if no improvement is found to be worthwhile in the section;

- Unacceptable Levels (UL) - Unacceptable conditions (ULs) must be corrected is requested by the user;

- Reconstruction Levels (RL) - RLs indicate the PSR for pavements below which the pavement must be reconstructed rather than resurfaced;

- Design Standards - If a section is reconstructed, then all design standards must be satisfied; and

- Minimum Tolerable Conditions (MTC) - Typically, MTCs fall between SDLs and ULs.

Deficiency levels are entered into input tables in HERS-ST. A sample HERS-ST pavement parameter input window is provided as Figure 2.3. Descriptions of the default deficiency levels are provided in Table F.1 of Appendix F. 


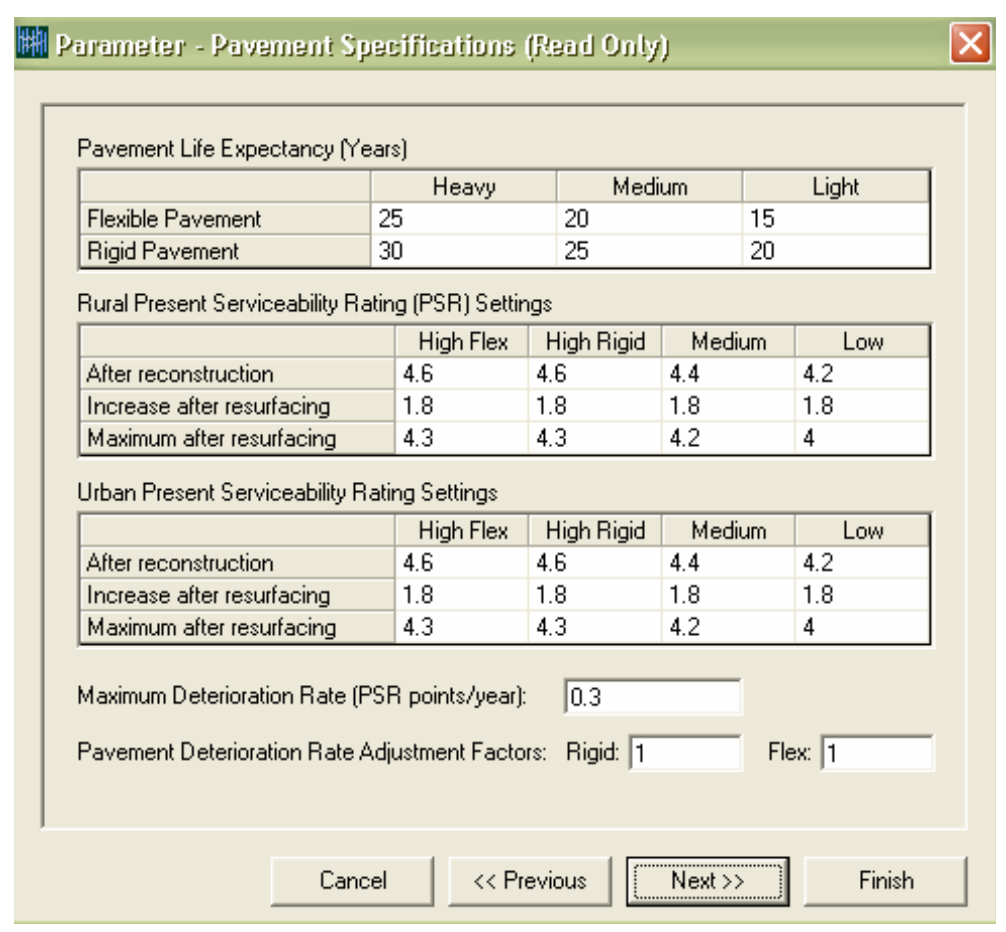

Figure 2.3 HERS-ST Pavement Specification Defaults for a Selected Scenario

\section{Pavement Deterioration Rate}

The analyst may specify (1) the PSR of newly constructed sections, (2) the increase in PSR due to resurfacing, and (3) the maximum PSR after resurfacing. In addition, the analyst may set the rate of pavement deterioration, making it possible to modify the pavement deterioration rate to reflect local conditions or alternative scenario assumptions. The maximum pavement deterioration rate per year may be adjusted as well. This value is significant for sections for which the database contains low structural numbers that would otherwise result in excessive deterioration rates. The default value for this maximum rate is 0.3 PSR per year.

\section{Pavement Condition}

Pavement conditions influence user costs, i.e., operating costs, safety, and travel time and are measured by PSR. Pavement with a PSR below 2.0 is generally not acceptable as a paved surface and is considered to have failed. Measurement of PSR requires visual inspection of the pavement and has been replaced (or supplemented) in the HPMS database by the International Roughness Index (IRI), a method based on physical measurements. HERS-ST accepts IRI measurements but converts them to the PSR scale for internal processing.

\subsubsection{HERS-ST Scenarios}


Two scenarios were run for various combinations of the maximum pavement deterioration per year (PDRMax) in PSR and the PSR increase (PSRInc) expected after resurfacing. Scenario A utilized a 0.3 PDRMax and 1.8 PSRInc., and Scenario B used a 0.4 PDRMax and 1.6 PSRInc. The needs presented below are for Scenario $A$ as this scenario was considered to provide more realistic results.

\subsubsection{HERS-ST Analysis and Results}

The HERS-ST analysis found that the 15-year highway infrastructure needs estimate for a state-controlled highway in Indiana is 17.1 to 17.4 billion dollars, using the two scenarios. The needs estimate is for pavements only. A summary of the initial and final condition of the state highway network based on the HERS-ST analysis for Scenario A is provided in Table 2.13. A summary of the change in lanes-miles, the total number of miles improved, the total lane-miles improved, and the total 15-year costs for improvements to these miles of highway are illustrated in Table 2.14. A total of 10,408 rural miles (39,026 lane-miles) were identified for improvement.

Table 2.13 State Highway System Pavement Condition Summary 


\begin{tabular}{|c|c|c|c|c|c|c|c|}
\hline \multirow[b]{2}{*}{ Location } & \multirow[b]{2}{*}{ Functional Class } & \multicolumn{3}{|c|}{ Initial Conditions } & \multicolumn{3}{|c|}{ Final Conditions } \\
\hline & & Miles & $\begin{array}{l}\text { Lane- } \\
\text { Miles }\end{array}$ & $\begin{array}{l}\text { Avg. } \\
\text { IRI }\end{array}$ & Miles & $\begin{array}{l}\text { Lane- } \\
\text { Miles }\end{array}$ & $\begin{array}{l}\text { Avg } \\
\text { IRI }\end{array}$ \\
\hline \multirow{5}{*}{ Rural } & Interstate & 852 & 3445 & 78 & 852 & 5235 & 66 \\
\hline & $\begin{array}{c}\text { Other Principal } \\
\text { Arterial }\end{array}$ & 1732 & 4730 & 79 & 1732 & 5363 & 70 \\
\hline & Major Arterial & 2149 & 4837 & 83 & 2149 & 5303 & 107 \\
\hline & Major Collector & 4708 & 9660 & 98 & 4708 & 10046 & 125 \\
\hline & Rural Subtotal & 9442 & 22673 & 83 & 9442 & 25949 & 84 \\
\hline \multirow{7}{*}{ Urban } & Interstate & 316 & 1548 & 104 & 316 & 4747 & 66 \\
\hline & Other & 126 & 512 & 99 & 126 & 788 & 66 \\
\hline & $\begin{array}{c}\text { Freeway/Express. } \\
\text { Other Principal } \\
\text { Arterial }\end{array}$ & 1011 & 3080 & 114 & 1011 & 4485 & 81 \\
\hline & Major Arterial & 223 & 494 & 107 & 223 & 558 & 111 \\
\hline & Collector & 0 & 0 & 121 & 0 & 0 & 151 \\
\hline & Urban Subtotal & 1677 & 5636 & 108 & 1677 & 10580 & 72 \\
\hline & Total All Locations & 11120 & 28310 & 93 & 11120 & 36530 & 79 \\
\hline
\end{tabular}

Table 2.14 2006-2020 Improvements and Costs for the State Highway System

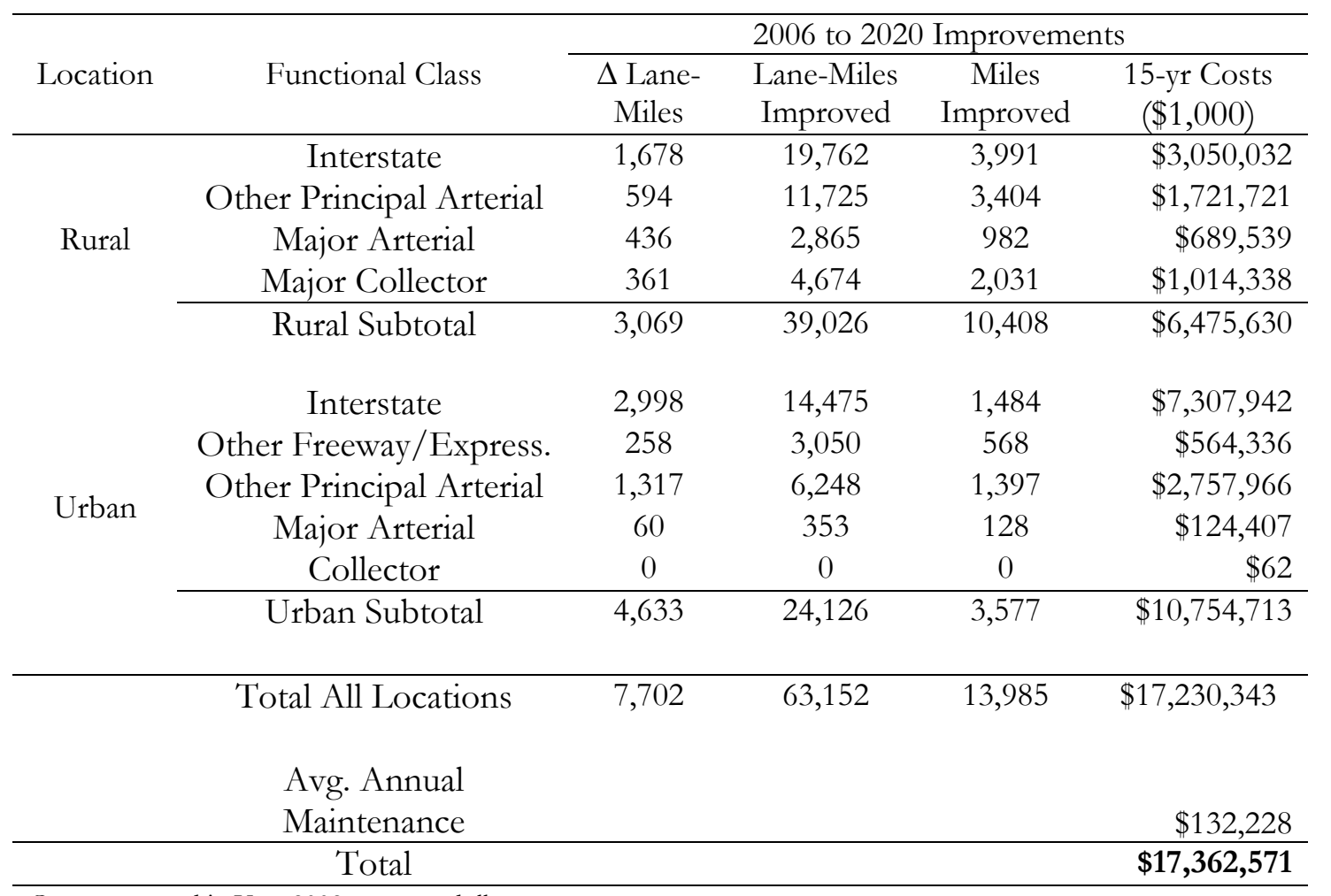

Costs expressed in Year 2002 constant dollars.

The total 15-year need for the rural locations is $\$ 6,457,630,000$ (\$Year 2002). The largest need by functional class is on the interstate system where the change in lane-miles in rural locations 
was 1,678 and 2,998 in urban areas. The 15-year need according to functional class of the highway is shown in Figure 2.4. Table 2.15 presents the 15 -year reconstruction needs by location. Figure 2.5 shows reconstruction needs by activity type, while Figure 2.6 gives reconstruction needs by highway type. Details of reconstruction needs are given in Table 2.16.

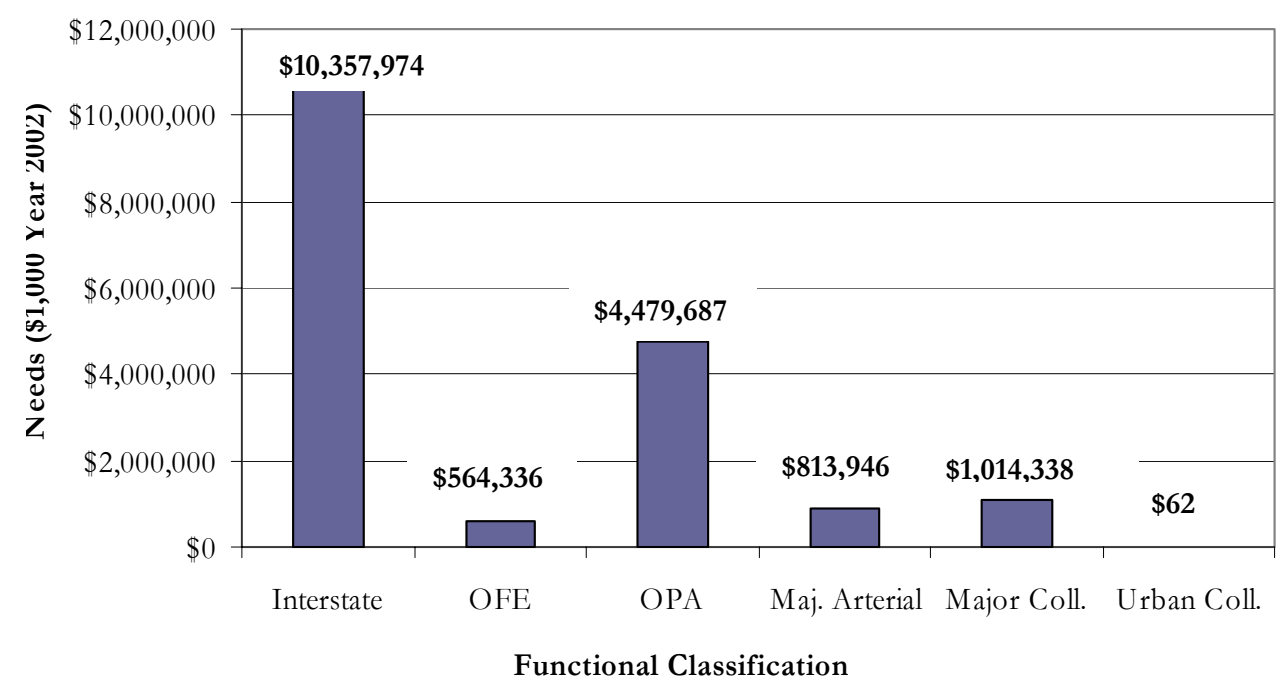

Figure 2.4 Fifteen -year Needs by Functional Classification

Table 2.15 Fifteen-year Reconstruction Needs by Location 


\begin{tabular}{cccccc}
\hline & & \multicolumn{3}{c}{2006 to 2020 Improvements $(\$ 1,000)$} \\
\hline Location & Functional Class & RCHC & RCNC & RCWL & RC \\
& Interstate & $\$ 0$ & $\$ 0$ & $\$ 0$ & $\$ 0$ \\
\multirow{4}{*}{ Rural } & Other Principal Arterial & $\$ 0$ & $\$ 0$ & $\$ 0$ & $\$ 2,637$ \\
& Major Arterial & $\$ 0$ & $\$ 0$ & $\$ 0$ & $\$ 0$ \\
& Major Collector & $\$ 0$ & $\$ 0$ & $\$ 0$ & $\$ 0$ \\
\cline { 2 - 6 } & Rural Subtotal & $\$ 0$ & $\$ 0$ & $\$ 0$ & $\$ 2,637$ \\
& Interstate & $\$ 0$ & $\$ 84,036$ & $\$ 0$ & $\$ 0$ \\
& Other & $\$ 0$ & $\$ 0$ & $\$ 0$ & $\$ 0$ \\
& Freeway/Expresswy & & & & \\
& Other Principal Arterial & $\$ 32,065$ & $\$ 11,576$ & $\$ 0$ & $\$ 3,959$ \\
& Major Arterial & $\$ 0$ & $\$ 0$ & $\$ 0$ & $\$ 0$ \\
Collector & $\$ 0$ & $\$ 0$ & $\$ 0$ & $\$ 0$ \\
\cline { 2 - 6 } & Urban Subtotal & $\$ 32,065$ & $\$ 95,612$ & $\$ 0$ & $\$ 3,959$ \\
& & \multicolumn{5}{c}{ Total Reconstruction } & $\$ 131,636$ \\
\hline
\end{tabular}

Costs expressed in Year 2002 constant dollars.

RCHC, pavement reconstruction and add high-cost lanes.

RCNC, pavement reconstruction and add normal-cost lanes.

RCWL, pavement reconstruction with wider lanes.

$\mathrm{RC}$, pavement reconstruction. 


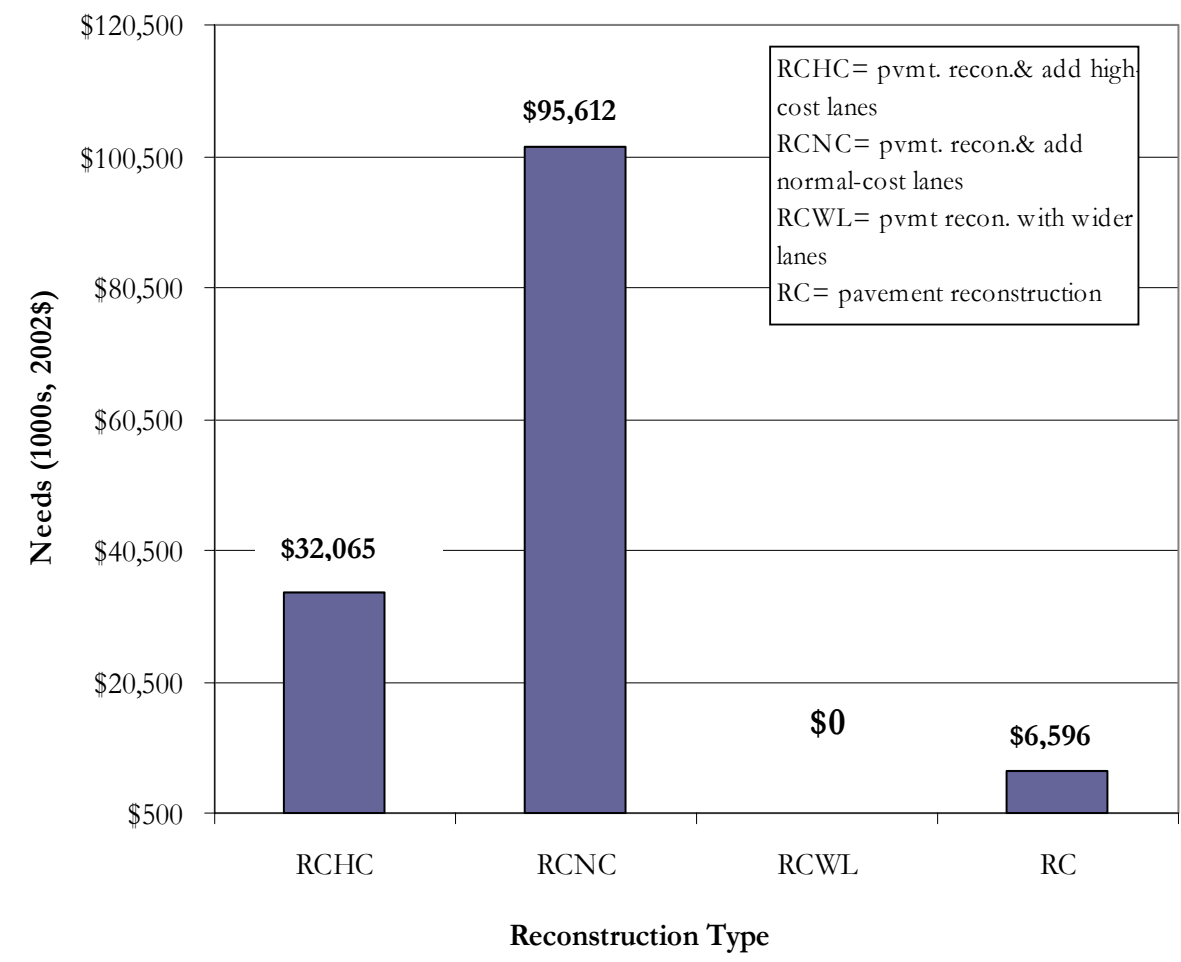

Figure 2.5 Fifteen -year Reconstruction Needs

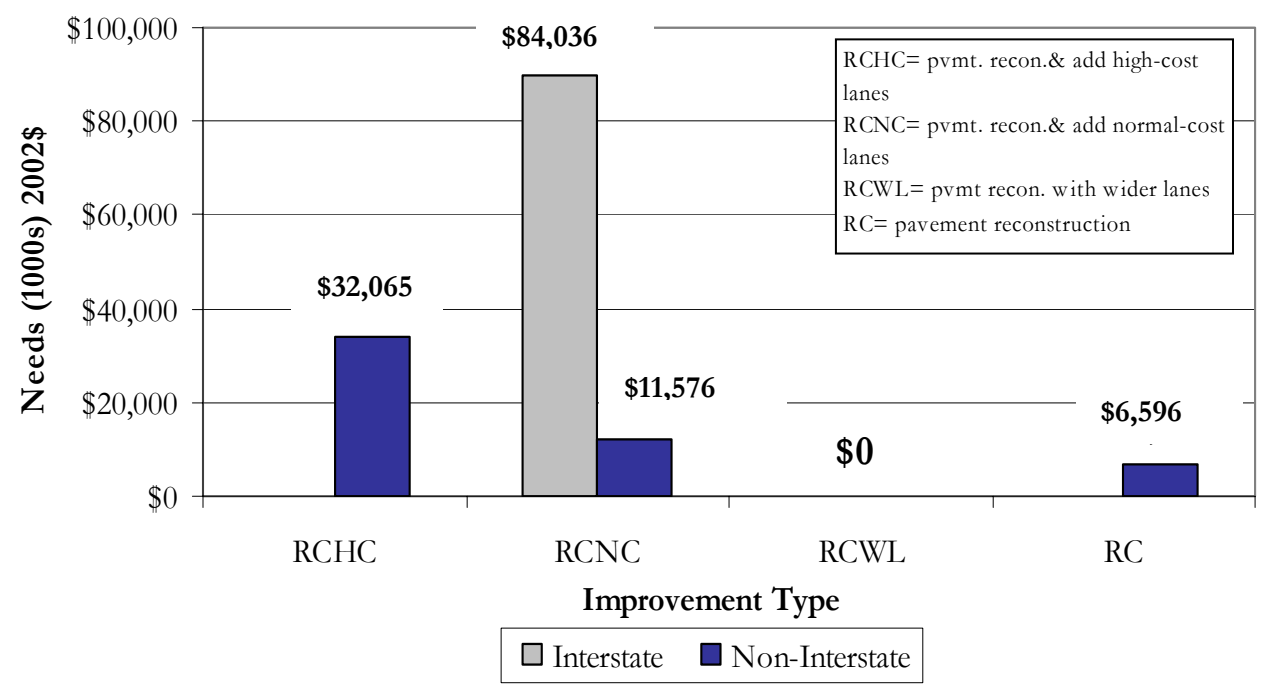

Figure 2.6 Interstate and Non-Interstate Fifteen -year Reconstruction Needs 
Table 2.16 Interstate and Non-Interstate Reconstruction Needs

\begin{tabular}{cccccc}
\hline \multirow{2}{*}{ Location } & Functional Class & RCHC & RCNC & RCWL & RC \\
\cline { 3 - 6 } & & & & & \\
\hline \multirow{2}{*}{ Rural } & Interstate & $\$ 0$ & $\$ 0$ & $\$ 0$ & $\$ 0$ \\
& Non-Interstate & $\$ 0$ & $\$ 0$ & $\$ 0$ & $\$ 2,637$ \\
& & & & $\$ 0$ & $\$ 0$ \\
Urban & Interstate & $\$ 0$ & $\$ 84,036$ & $\$ 0$ & $\$ 3,959$ \\
& Non-Interstate & $\$ 32,065$ & $\$ 11,576$ & $\$ 0$ & \\
\hline Total & Interstate & $\mathbf{\$ 0}$ & $\mathbf{\$ 8 4 , 0 3 6}$ & $\mathbf{\$ 0}$ & $\mathbf{\$ 0}$ \\
& Non-Interstate & $\mathbf{\$ 3 2 , 0 6 5}$ & $\mathbf{\$ 1 1 , 5 7 6}$ & $\mathbf{\$ 0}$ & $\mathbf{\$ 6 , 5 9 6}$ \\
\hline
\end{tabular}

The 15-year need for pavement reconstruction with no added-lanes (RC) is $\$ 6,596,000$. The 15 -year need for pavement reconstruction with added normal cost lanes (RCNC) is $\$ 11,576,000$. The 15-year need for pavement reconstruction with high cost lanes (RCHC) is $\$ 32,065,000$. The Scenario A resurfacing needs by location and highway class are shown in Table 2.17. Total resurfacing needs under Scenario A are $\$ 17,097,504$. Figure 2.7 presents these needs by functional class, while Figure 2.8 summarizes the need amounts by activity type. Figure 2.9 presents resurfacing needs by highway type and the details of resurfacing needs are given in Table 2.18.

Table 2.17 Scenario A Resurfacing Needs by Location and Functional Class

\begin{tabular}{|c|c|c|c|c|c|c|}
\hline \multirow[b]{2}{*}{ Location } & \multirow[b]{2}{*}{ Functional Class } & \multicolumn{5}{|c|}{2006 to 2020 Improvements $(\$ 1,000)$} \\
\hline & & RSHC & RSNC & RSWL & RSSH & RS \\
\hline \multirow{5}{*}{ Rural } & Interstate & $\$ 0$ & $\$ 1,553,932$ & $\$ 0$ & $\$ 0$ & $\$ 1,496,100$ \\
\hline & $\mathrm{OPA}^{\mathrm{a}}$ & $\$ 201,638$ & $\$ 366,411$ & $\$ 98,313$ & $\$ 458,504$ & $\$ 594,647$ \\
\hline & Major Arterial & $\$ 223,881$ & $\$ 145,223$ & $\$ 180,202$ & $\$ 72,992$ & $\$ 67,242$ \\
\hline & Major Collector & $\$ 174,955$ & $\$ 124,889$ & $\$ 575,773$ & $\$ 107,555$ & $\$ 31,166$ \\
\hline & Rural Subtotal & $\$ 600,474$ & $\$ 2,190,455$ & $\$ 854,288$ & $\$ 639,051$ & $\$ 2,189,155$ \\
\hline \multirow{7}{*}{ Urban } & Interstate & $\$ 2,579,302$ & $\$ 3,542,696$ & $\$ 0$ & $\$ 202$ & $\$ 1,101,707$ \\
\hline & $\mathrm{OFE}^{\mathrm{b}}$ & $\$ 0$ & $\$ 304,603$ & $\$ 563$ & $\$ 20,642$ & $\$ 238,528$ \\
\hline & $\mathrm{OPA}^{\mathrm{a}}$ & $\$ 1,866,351$ & $\$ 324,858$ & $\$ 176,096$ & $\$ 123,167$ & $\$ 219,894$ \\
\hline & Major Arterial & $\$ 78,661$ & $\$ 8,716$ & $\$ 13,212$ & $\$ 14,574$ & $\$ 9,243$ \\
\hline & Collector & $\$ 0$ & $\$ 0$ & $\$ 0$ & $\$ 66$ & $\$ 0$ \\
\hline & Urban Subtotal & $\$ 4,525,314$ & $\$ 4,180,873$ & $\$ 189,871$ & $\$ 158,651$ & $\$ 1,569,372$ \\
\hline & & & & & Total & $\$ 17,097,504$ \\
\hline
\end{tabular}

Costs expressed in Year 2002 constant dollars.

a. $\mathrm{OPA}=$ Other Principal Arterial

b. OFE $=$ Other Freeways and Expressways

RS, resurface.

$\mathrm{RSSH}$, resurface and improve shoulders.

RSWL, resurface and widen lanes (minor widening).

RSNC, resurface and add normal-cost lanes (major widening).

RSHC, resurface and add high-cost lanes. 


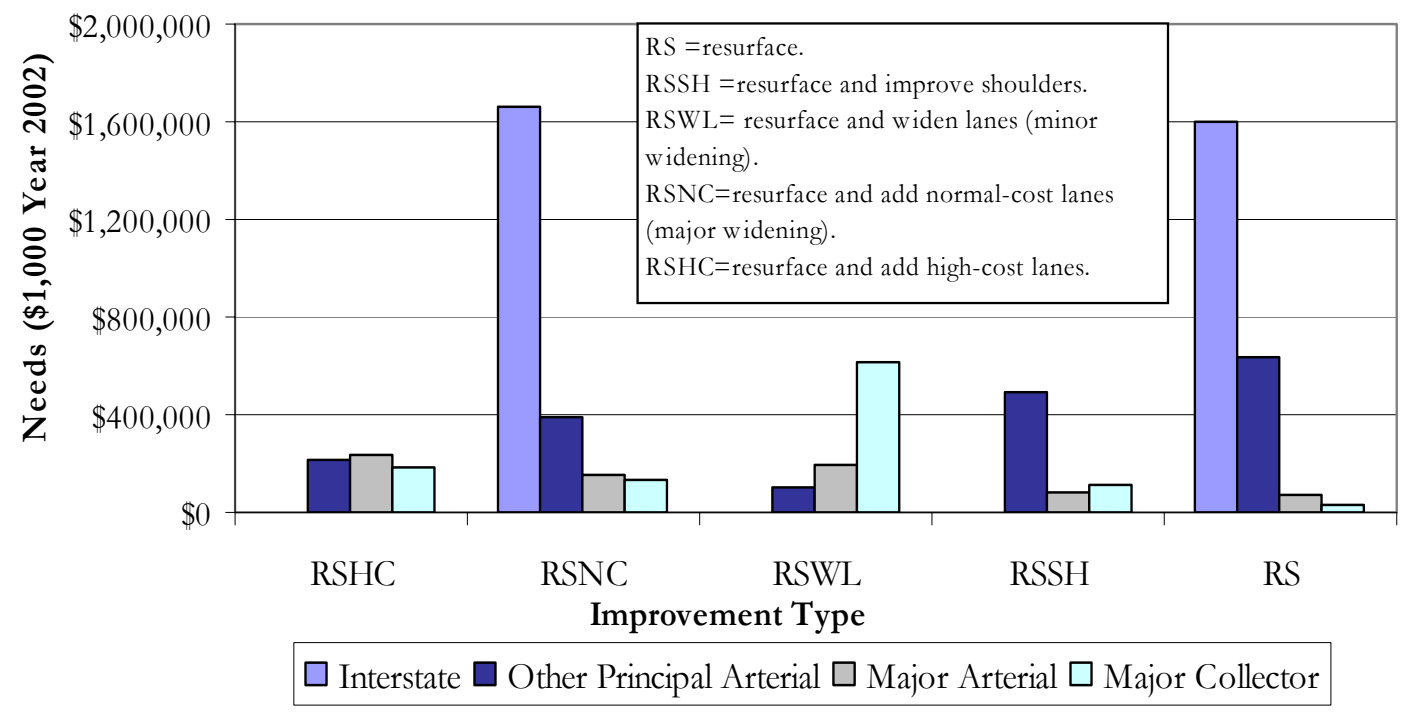

Figure 2.7 Fifteen -year Rural Road Resurfacing Needs by Functional Class

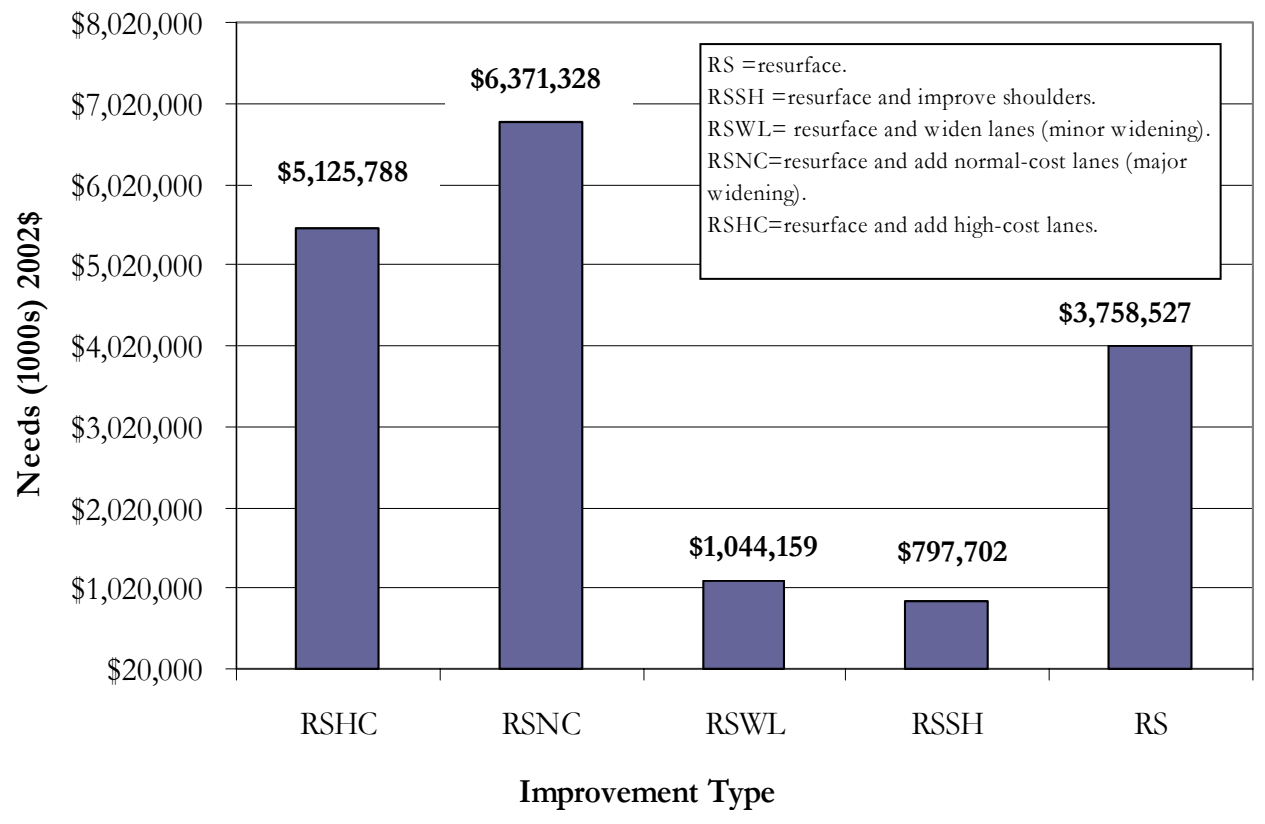

Figure 2.8 Fifteen -year Total Resurfacing Needs 


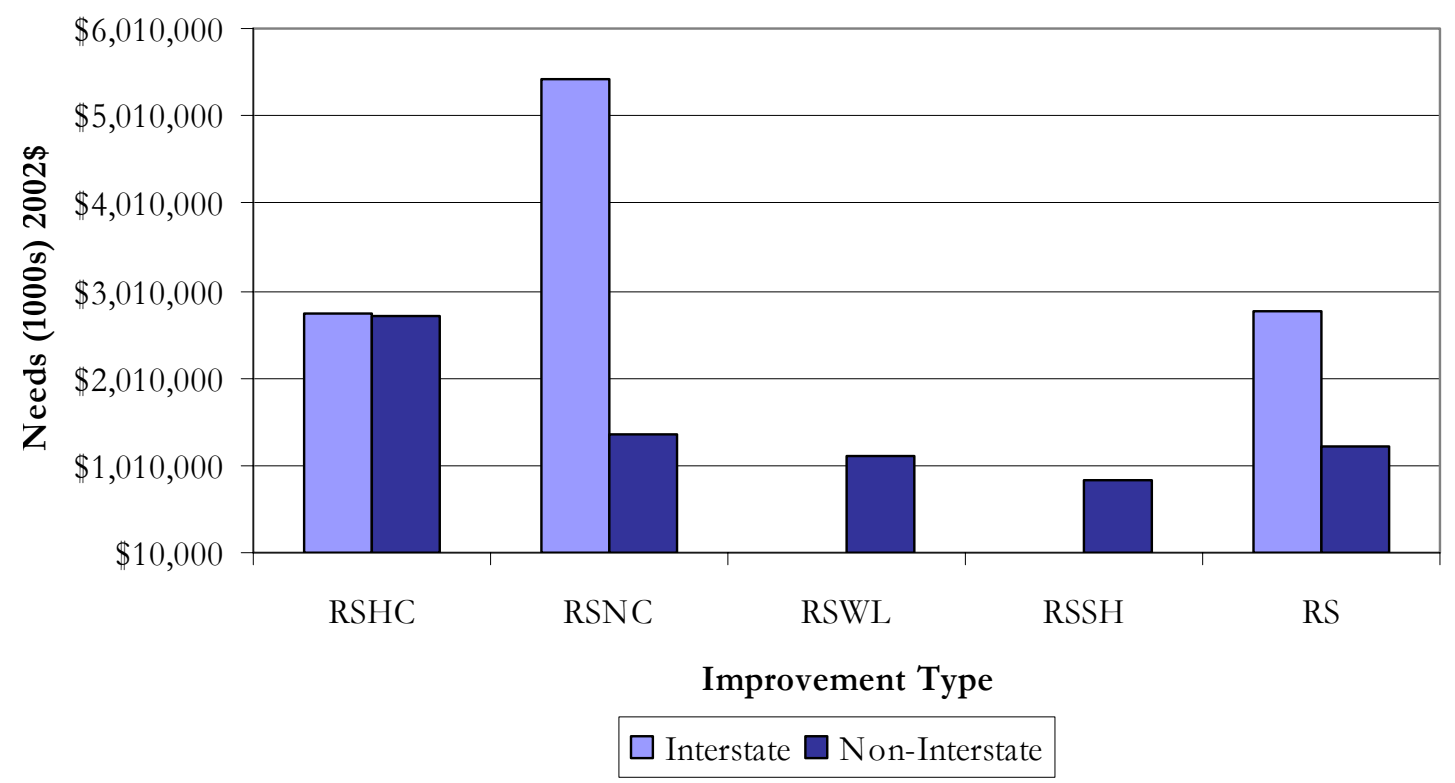

Figure 2.9 Fifteen -year Interstate and Non-Interstate Resurfacing Needs

Table 2.18 Fifteen-year Interstate and Non-Interstate Resurfacing Needs

\begin{tabular}{ccccccc}
\hline \multirow{2}{*}{ Location } & Functional & \multicolumn{5}{c}{2006 to 2020 Improvements $(\$ 1,000)$} \\
\cline { 2 - 7 } & Class & RSHC & RSNC & RSWL & RSSH & RS \\
\cline { 3 - 7 } Rural & Interstate & $\$ 0$ & $\$ 1,553,932$ & $\$ 0$ & $\$ 0$ & $\$ 1,496,100$ \\
& Non-Interstate & $\$ 600,474$ & $\$ 636,096$ & $\$ 854,288$ & $\$ 639,050$ & $\$ 693,054$ \\
& & & & & & \\
\multirow{2}{*}{ Urban } & Interstate & $\$ 2,579,302$ & $\$ 3,542,696$ & $\$ 0$ & $\$ 202$ & $\$ 1,101,707$ \\
& Non-Interstate & $\$ 1,945,012$ & $\$ 638,175$ & $\$ 189,872$ & $\$ 158,446$ & $\$ 467,665$ \\
& & & & & & \\
\hline \multirow{2}{*}{ Total } & Interstate & $\mathbf{\$ 2 , 5 7 9 , 3 0 2}$ & $\mathbf{\$ 5 , 0 9 6 , 6 2 8}$ & $\mathbf{\$ 0}$ & $\mathbf{\$ 2 0 2}$ & $\mathbf{\$ 2 , 5 9 7 , 8 0 7}$ \\
& Non-Interstate & $\mathbf{\$ 2 , 5 4 5 , 4 8 6}$ & $\mathbf{\$ 1 , 2 7 4 , 2 7 1}$ & $\mathbf{\$ 1 , 0 4 4 , 1 6 0}$ & $\mathbf{\$ 7 9 7 , 4 9 6}$ & $\mathbf{\$ 1 , 1 6 0 , 7 9 1}$ \\
\hline
\end{tabular}

Costs expressed in Year 2002 constant dollars.

\subsubsection{HERS-ST Summary and Conclusions}

The HERS-ST analysis found that the 15-year needs for pavement, capacity, and safety were in the range of $\$ 17,097,504,000$ and $\$ 17,362,571,000$. These costs exclude the costs for new roads and bridges planned between 2006 and 2020. 


\subsubsection{Manual Network Condition Analysis}

The network condition analysis was used as a manual check of the HERS-ST results for resurfacing and reconstruction pavement needs. This section describes the data and methodology used for the network condition analysis approach. The unit pavement treatment costs developed by Lamptey et al. (2004), as shown in Table 2.9, and the average annual maintenance expenditure costs (Labi and Sinha, 2003), given in Table 2.11, were used for this analysis.

Two pavement deterioration rates were used in the analysis: 6 IRI and 8 IRI per year. The analysis used IRI pavement condition levels as a trigger to initiate specific pavement treatments based on the pavement condition ranges established by INDOT. All lane-miles were treated with regular annual maintenance, using the AAMEX curves developed by Labi and Sinha (2003).

The data used in the manual method did not include information on shoulders, capacity, or alignment deficiencies, and the need estimates therefore are based solely on improvements to the mainline pavement and do not include costs for improvements in alignments, shoulder, or capacity. Capacity improvement needs, such as pavement widening, are based on those identified in the INDOT LRP. Safety needs in this manual method are based on an estimate of 9.4 million dollars per year, in year 2002 constant dollars, for road segments only (excluding intersections) as identified in the research project by Lamptey et al. (2004), which utilized the Indiana Safety and Congestion Management Systems Software. For the present study, the safety improvement need at intersections was based on improvements identified in the LRP.

\section{Collection and Processing of Data}

The 2001 pavement contracts database used in the research by Lamptey et al. (2004) was obtained for use as a source of pavement condition data. The contracts database was sorted to group the pavements into three main categories: HMA, PCC, and Composite (HMA over PCC). The pavements were then separated by location: Interstate, Non-Interstate NHS (on the National Highway System), and Non-Interstate Non-NHS. The needs analysis was conducted on the pavements based on these categories of pavement type and location.

\section{Methodology}

The same pavement condition threshold levels used in HERS-ST analysis were used to trigger pavement treatments. Tables 2.19 and 2.20 illustrate the equivalent IRI deficiency thresholds used for resurfacing and reconstruction treatments in the manual analysis method based on the equivalent deficiency thresholds used for HERS-ST. 
Table 2.19 Network Analysis Pavement Resurfacing Deficiency Levels

\begin{tabular}{ccc}
\hline Location & $\begin{array}{c}\text { INDOT IRI } \\
\text { Equivalent }\end{array}$ & $\begin{array}{c}\text { HERS Deficiency } \\
\text { bevel (PSR) }\end{array}$ \\
\hline Interstate & 118 & 3.2 \\
\hline Principal Arterial AADT $>6000$ & 118 & 3.2 \\
\hline Principal Arterial AADT $<6000$ & 126 & 3.0 \\
\hline Minor Arterial AADT $>2000$ & 142 & 2.6 \\
\hline Minor Arterial AADT $<2000$ & 142 & 2.6 \\
\hline Major Collector AADT $>1000$ & 151 & 2.4 \\
\hline Major Collector AADT $>400$ & 151 & 2.4 \\
\hline Major Collector AADT $<400$ & 161 & 2.2 \\
\hline Urban Interstate & 111 & 3.4 \\
\hline Urban Freeway & 118 & 3.2 \\
\hline Urban Principal Arterial & 126 & 3.0 \\
\hline Urban Minor Arterial & 142 & 2.6 \\
\hline Urban Collectors & 151 & 2.4 \\
\hline
\end{tabular}

a. Calculated using Gulen (1994).

b. Default values from HERS-ST.

According to Table 2.19, a 3.2 PSR for a principal arterial is equivalent to 118 IRI, which means that if a pavement condition falls below 118 IRI, then resurfacing is implemented. For urban minor arterials, if the IRI falls below 142, then the pavement is resurfaced. Similarly, in Table 2.20, if an urban interstate has an IRI of less than 161, it receives pavement reconstruction.

Table 2.20 Network Analysis Pavement Reconstruction Deficiency Levels

\begin{tabular}{ccc}
\hline Location & $\begin{array}{c}\text { INDOT IRI } \\
\text { Equivalent }\end{array}$ & $\begin{array}{c}\text { HERS Deficiency } \\
\text { Level (PSR) }\end{array}$ \\
\hline Interstate & 172 & 2 \\
\hline Principal Arterial AADT $>6000$ & 172 & 2 \\
\hline Principal Arterial AADT $<6000$ & 172 & 2 \\
\hline Minor Arterial AADT $>2000$ & 205 & 1.5 \\
\hline Minor Arterial AADT $<2000$ & 205 & 1.5 \\
\hline Major Collector AADT $>1000$ & 221 & 1.3 \\
\hline Major Collector AADT $>400$ & 221 & 1.3 \\
\hline Major Collector AADT $<400$ & 240 & 1.1 \\
\hline Urban Interstate & 161 & 2.2 \\
\hline Urban Freeway & 172 & 2 \\
\hline Urban Principal Arterial & 184 & 1.8 \\
\hline Urban Minor Arterial & 221 & 1.1 \\
\hline Urban Collector & 240 &
\end{tabular}

After sorting the pavement data by location and pavement material type, the data was analyzed to determine the number of lane-miles that fell within the INDOT-specified IRI ranges 
from excellent to poor. The good-to-excellent category was split into an upper and lower range of good condition to create the option for additional pavement treatments for pavements in the goodto-excellent range. The treatment IRI ranges were:

- Excellent - Good, IRI $=60-100$

- $\quad$ Good, IRI=101 --125

- $\quad$ Good - Fair, IRI = $126--150$

- $\quad$ Fair, IRI $=151-200$

- $\quad$ Poor, $\mathrm{IRI}=>200$

The initial IRI is the condition of the pavement at the time of the pavement condition survey in year 2001. The initial pavement IRI was compared to the established trigger values that would indicate the need for a resurfacing project, a reconstruction project, or a "do nothing" option. Annual maintenance was applied each year to all pavements. The research by Lamptey et al. (2004) determined the average increase in pavement condition or "performance jump" after specific treatments were applied based on historical data from the contracts database. Two treatments used in the network analysis utilized the performance jumps identified by Lamptey et al., specifically, thin overlays and cleaning and sealing of joints on portland cement concrete pavement (PCCP). The average pavement performance jump associated with these treatments is illustrated in Table 2.21. Forty-two pavement sections were used in the analysis of the pavement jump associated with HMA overlays and 18 sections of pavement data were used to determine the pavement performance jumps associated with cleaning and sealing of joints on concrete pavement. Based on the performance jump information provided by Lamptey et al. (2004), the condition of the pavement should improve by 53.3 IRI after an HMA overlay and improve by 22.7 IRI after cleaning and sealing of joints on PCC pavement.

Table 2.21 Pavement Performance (IRI) Jumps after Treatment

\begin{tabular}{lcc}
\hline \multicolumn{1}{c}{ Treatment Type } & $\begin{array}{c}\text { Average } \\
\text { Jump }\end{array}$ & $\begin{array}{c}\text { No. of } \\
\text { Sections }\end{array}$ \\
\hline HMA Overlay, Preventive Maintenance & 53.5 & 42 \\
PCCP Cleaning and Sealing Joints & 22.7 & 18 \\
\hline
\end{tabular}

Lamptey et al. (2004).

Pavements with less than 126 IRI at the start of the analysis were treated so as keep all of them in that condition over the 15-year analysis period. In essence, any pavements in excellent condition at the start of the analysis therefore would be maintained in that excellent condition category. The trigger value for work on pavements with initial IRI of $<126$ was 118 IRI. Specific treatments were selected to represent the typical treatments to be applied to pavements based on their IRI. Flexible pavements (HMA) in good to fair condition (IRI 126 to 150) would be treated 
with preventive maintenance or a thin HMA overlay specifically, while PCC pavements in good to fair condition would be treated with joint and crack sealing. The typical treatment for pavements in fair condition (IRI 151-200) was the Resurfacing Partial 3-R standards, while those in poor condition would be replaced or reconstructed. Any pavement that received a Resurfacing Partial 3-R standards or Reconstruction as the initial treatment was assumed to be returned to excellent condition, specifically 60 IRI for HMA pavements and 70 IRI for concrete pavements, which are the accepted IRI conditions for new pavements of those material types.

\section{Interstate Initial Pavement Condition and Treatment}

A total of 4,143 lane-miles of interstate were included in the analysis. A summary of the initial pavement conditions, as well as the initial treatment chosen for the interstate pavement data, are provided in Table 2.22. Approximately, 92 percent of the interstate system was in excellent condition; 97 percent was in excellent or good condition; and there were no lane-miles on the interstate in poor condition. Figure 2.10 illustrates the interstate pavement condition in year 2001.

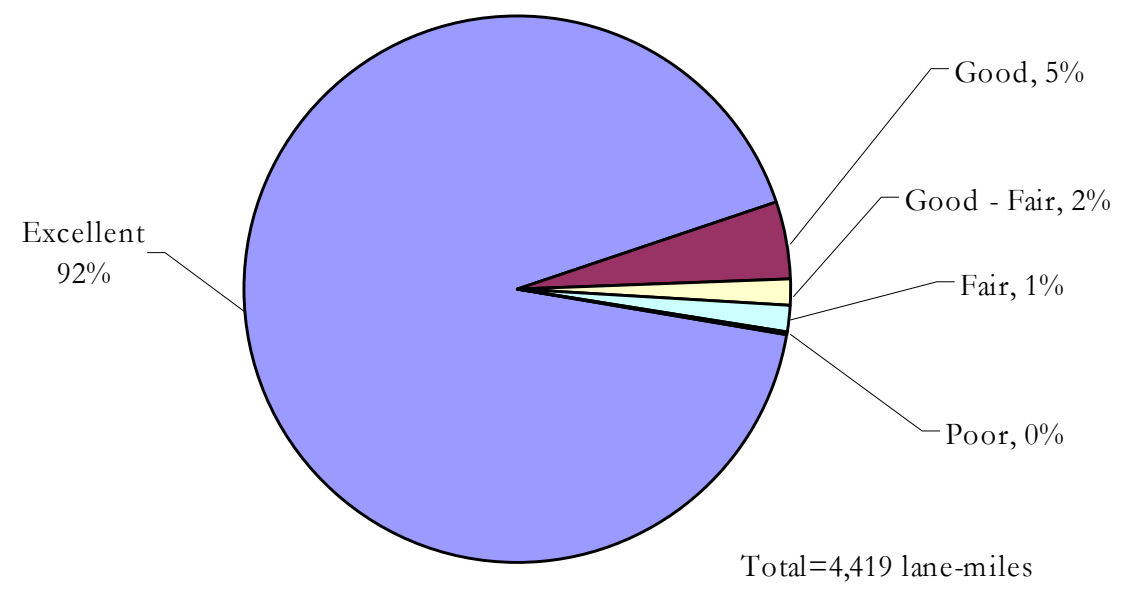

Figure 2.10 Distribution of Interstate Pavement as of Year 2001 
Table 2.22 Interstate HMA Pavement Treatment Thresholds

\begin{tabular}{|c|c|c|c|c|}
\hline Condition (IRI) & $\begin{array}{l}\text { Deficiency } \\
\text { Level IRI }\end{array}$ & $\begin{array}{c}\text { Existing } \\
\text { Average IRI }\end{array}$ & $\begin{array}{l}\text { Lane- } \\
\text { miles }\end{array}$ & $\begin{array}{l}\text { "Initial" Treatment } \\
\text { (Year 2001) }\end{array}$ \\
\hline $\begin{array}{l}\text { Excellent-Good } \\
(\text { IRI }=60-100)\end{array}$ & Trigger $>118$ & 71 & 658 & Do Nothing \\
\hline $\begin{array}{c}\text { Good } \\
(\text { IRI }=101-125)\end{array}$ & Trigger $>118$ & 106 & 11 & Do Nothing \\
\hline $\begin{array}{c}\text { Good }- \text { Fair } \\
(\text { IRI }=126-150)\end{array}$ & Trigger $>134$ & 145 & 11 & $\begin{array}{c}\text { Preventive } \\
\text { Maintenance }\end{array}$ \\
\hline $\begin{array}{c}\text { Fair } \\
(I R I=151-200)\end{array}$ & Trigger $>151$ & 155 & 1 & $\begin{array}{c}\text { Resurfacing Partial 3-R } \\
\text { Standards }\end{array}$ \\
\hline $\begin{array}{c}\text { Poor } \\
(I R I=>200)\end{array}$ & Trigger $>200$ & - & 0 & Reconstruction \\
\hline All Conditions & $\mathrm{n} / \mathrm{a}$ & $\mathrm{n} / \mathrm{a}$ & 681 & $\begin{array}{l}\text { Maintenance } \\
\text { (AAMEX) }\end{array}$ \\
\hline
\end{tabular}

In year 2001, there were no treatments applied to 669 lane-miles of highway because their initial IRI exceeded 118 IRI (the trigger value). Eleven lane-miles in the good-fair pavement condition range met the criteria for treatment as their average IRI was 145 with the trigger value for treatment was an IRI greater than 134. A thin overlay preventive maintenance treatment was applied to these sections in year 2001, thereby decreasing their IRI by 53.5 units. In separate scenarios the pavements were simulated to deteriorate by six or eight IRI and when they again reached a condition that exceeded 134 IRI another treatment of thin overlay was simulated, thereby keeping the pavements in good-to-fair condition at the start of the analysis from deteriorating to the lower condition levels such as fair or poor.

Tables 2.23 and 2.24 illustrate the initial treatments applied to the PCC and COMP interstate pavements. The methodology used for the treatment of pavements that were initially in excellent condition, such as the interstate pavements, as well as for the good to fair pavements is illustrated in Figure 2.11. 
Table 2.23 Interstate PCC Pavement Treatment Thresholds

\begin{tabular}{ccccc}
\hline Condition (IRI) & $\begin{array}{c}\text { Deficiency } \\
\text { Level IRI }\end{array}$ & $\begin{array}{c}\text { Existing } \\
\text { Average IRI }\end{array}$ & $\begin{array}{c}\text { Lane- } \\
\text { miles }\end{array}$ & $\begin{array}{c}\text { "Initial" Treatment } \\
\text { (Year 2001) }\end{array}$ \\
\hline $\begin{array}{c}\text { Excellent-Good } \\
\text { IRI }=60-100)\end{array}$ & Trigger $>118$ & 82 & 645 & Do Nothing \\
\hline $\begin{array}{c}\text { Good } \\
(\text { IRI }=101-125)\end{array}$ & Trigger $>118$ & 112 & 149 & Do Nothing \\
\hline $\begin{array}{c}\text { Good }- \text { Fair } \\
\text { (IRI }=126-150)\end{array}$ & Trigger $>134$ & 135 & 37 & Joint and Crack Sealing \\
\hline $\begin{array}{c}\text { Fair } \\
\text { (IRI }=151-200)\end{array}$ & Trigger $>151$ & 167 & 55 & $\begin{array}{c}\text { Resurfacing Partial 3-R } \\
\text { Standards }\end{array}$ \\
\hline $\begin{array}{c}\text { Poor } \\
(\text { IRI }=>200)\end{array}$ & Trigger $>200$ & 201 & 5 & Reconstruction \\
\hline All Conditions & $\mathrm{n} / \mathrm{a}$ & $\mathrm{n} / \mathrm{a}$ & 892 & Maintenance (AAMEX) \\
\hline
\end{tabular}

Table 2.24 Interstate COMP Pavement Treatment Thresholds

\begin{tabular}{|c|c|c|c|c|}
\hline Condition (IRI) & $\begin{array}{l}\text { Deficiency } \\
\text { Level IRI }\end{array}$ & $\begin{array}{c}\text { Existing } \\
\text { Average IRI }\end{array}$ & Lane-miles & $\begin{array}{c}\text { "Initial" Treatment } \\
\text { (Year 2001) }\end{array}$ \\
\hline $\begin{array}{l}\text { Excellent-Good } \\
(\text { IRI }=60-100)\end{array}$ & Trigger $>118$ & 73 & 2771 & Do Nothing \\
\hline $\begin{array}{c}\text { Good } \\
\text { (IRI=101 -125) }\end{array}$ & Trigger $>118$ & 114 & 41 & Do Nothing \\
\hline $\begin{array}{c}\text { Good }- \text { Fair } \\
(\text { IRI = } 126-150)\end{array}$ & Trigger $>134$ & 132 & 27 & $\begin{array}{c}\text { Preventive } \\
\text { Maintenance } \\
\end{array}$ \\
\hline $\begin{array}{c}\text { Fair } \\
(\text { IRI }=151-200)\end{array}$ & Trigger $>151$ & 163 & 5 & $\begin{array}{c}\text { Resurfacing Partial } \\
\text { 3-R Standards }\end{array}$ \\
\hline $\begin{array}{c}\text { Poor } \\
(\text { IRI }=>200)\end{array}$ & Trigger $>200$ & 218 & 2 & Reconstruction \\
\hline All Conditions & $\mathrm{n} / \mathrm{a}$ & $\mathrm{n} / \mathrm{a}$ & 2846 & $\begin{array}{l}\text { Maintenance } \\
\text { (AAMEX) }\end{array}$ \\
\hline
\end{tabular}




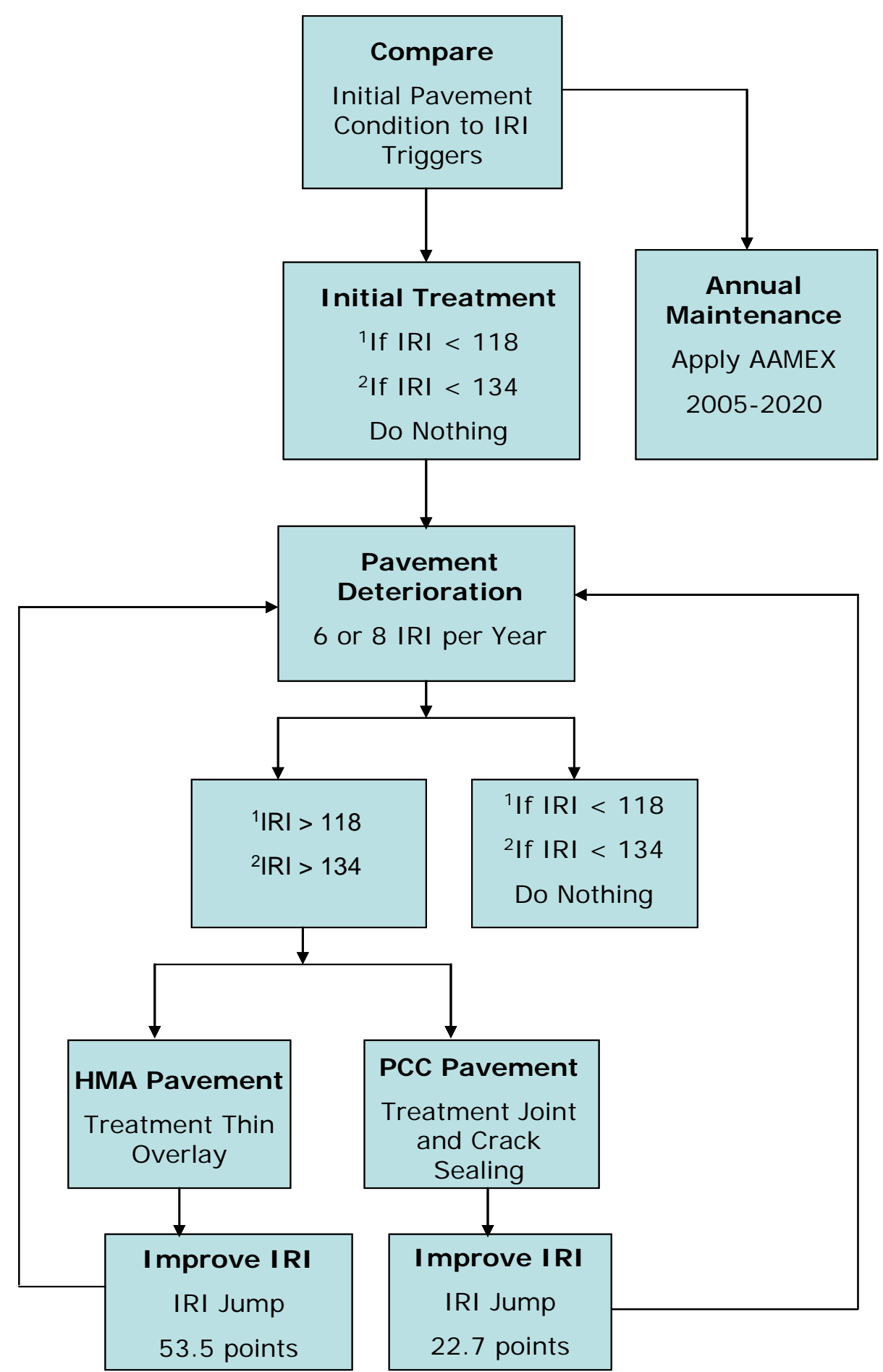

Figure 2.11 Methodology used for Excellent, Good and Good-To-Fair Pavements

${ }^{1}$ Excellent and Good Pavement

${ }^{2}$ Good-To-Fair Pavements 


\section{Non-Interstate NHS Pavement Condition and Initial Treatment}

This group of pavements represents 4,814 lane-miles of highway. Approximately 76 percent of non-interstate NHS pavements were in excellent condition and there were no sections in poor condition. A summary of the pavement conditions is illustrated in Figure 2.12, and the "initial" treatments applied to the pavements are described in Table 2.25, Table 2.26, and Table 2.27 for HMA, PCC and COMP pavement "initial" treatment respectively. "Initial" treatment is not a treatment at year 0, but rather the assumed treatment at the start of the year 2001.

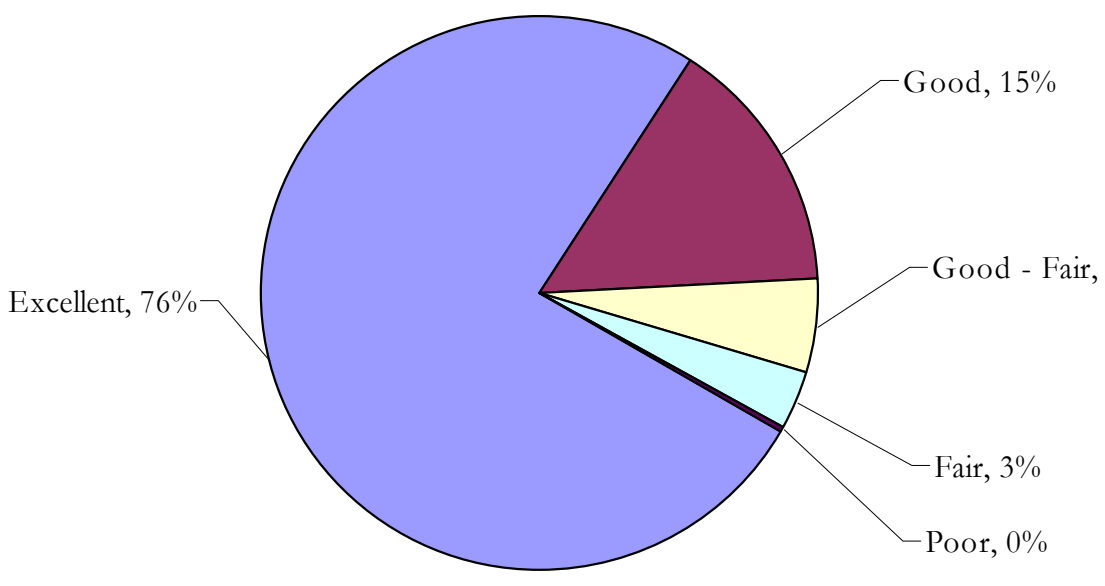

Total $=5,135$ lane-miles

Figure 2.12 Distribution of Non-Interstate NHS by Pavement Condition as of Year 2001

Table 2.25 Non-Interstate NHS HMA Pavement Treatment Thresholds

\begin{tabular}{ccccc}
\hline Condition (IRI) & $\begin{array}{c}\text { Deficiency } \\
\text { Level IRI }\end{array}$ & $\begin{array}{c}\text { Existing } \\
\text { Average IRI }\end{array}$ & Lane-miles & $\begin{array}{c}\text { "Initial" Treatment } \\
\text { (Year 2001) }\end{array}$ \\
\hline $\begin{array}{c}\text { Excellent-Good } \\
(\text { IRI }=60-100)\end{array}$ & Trigger $>118$ & 76 & 3267 & Do Nothing \\
\hline $\begin{array}{c}\text { Good } \\
(\text { IRI }=101-125)\end{array}$ & Trigger $>118$ & 111 & 596 & Do Nothing \\
\hline $\begin{array}{c}\text { Good }- \text { Fair } \\
\text { IRI }=126-150)\end{array}$ & Trigger $>134$ & 135 & 180 & $\begin{array}{c}\text { Preventive } \\
\text { Maintenance }\end{array}$ \\
\hline $\begin{array}{c}\text { Fair } \\
\text { (IRI }=151-200)\end{array}$ & Trigger $>151$ & 167 & 135 & $\begin{array}{c}\text { Resurfacing Partial } \\
\text { 3-R Standards }\end{array}$ \\
\hline $\begin{array}{c}\text { Poor } \\
\text { IRI }=>200)\end{array}$ & Trigger $>200$ & 230 & 8 & $\begin{array}{c}\text { Reconstruction } \\
\text { All Conditions }\end{array}$ \\
$\mathrm{n} / \mathrm{a}$ & $\mathrm{n} / \mathrm{a}$ & 4186 & $\begin{array}{c}\text { Maintenance } \\
\text { (AAMEX) }\end{array}$ \\
\hline
\end{tabular}


Table 2.26 Non-Interstate NHS PCC Pavement Treatment Thresholds

\begin{tabular}{ccccc}
\hline Condition (IRI) & $\begin{array}{c}\text { Deficiency } \\
\text { Level IRI }\end{array}$ & $\begin{array}{c}\text { Existing } \\
\text { Average IRI }\end{array}$ & $\begin{array}{c}\text { Lane- } \\
\text { miles }\end{array}$ & $\begin{array}{c}\text { "Initial" Treatment } \\
\text { (Year 2001) }\end{array}$ \\
\hline $\begin{array}{c}\text { Excellent-Good } \\
\text { (IRI }=60-100)\end{array}$ & Trigger $>118$ & 76 & 511 & Do Nothing \\
\hline $\begin{array}{c}\text { Good } \\
(\text { IRI }=101-125)\end{array}$ & Trigger $>118$ & 112 & 170 & Do Nothing \\
\hline $\begin{array}{c}\text { Good }- \text { Fair } \\
\text { IRI }=126-150)\end{array}$ & Trigger $>134$ & 139 & 108 & $\begin{array}{c}\text { Joint and Crack } \\
\text { Sealing }\end{array}$ \\
\hline $\begin{array}{c}\text { Fair } \\
(\text { IRI }=151-200)\end{array}$ & Trigger $>151$ & 162 & 35 & $\begin{array}{c}\text { Resurfacing Partial } \\
\text { 3-R Standards }\end{array}$ \\
\hline $\begin{array}{c}\text { Poor } \\
(\text { IRI }=>200)\end{array}$ & Trigger $>200$ & $\mathrm{n} / \mathrm{a}$ & 0 & $\begin{array}{c}\text { Reconstruction } \\
\text { All Conditions }\end{array}$ \\
$\mathrm{n} / \mathrm{a}$ & $\mathrm{n} / \mathrm{a}$ & 825 & $\begin{array}{c}\text { Maintenance } \\
\text { (AAMEX) }\end{array}$ \\
\hline
\end{tabular}

Table 2.27 Non-Interstate NHS COMP Pavement Treatment Thresholds

\begin{tabular}{|c|c|c|c|c|}
\hline Condition (IRI) & $\begin{array}{l}\text { Deficiency } \\
\text { Level IRI }\end{array}$ & $\begin{array}{c}\text { Existing } \\
\text { Average IRI }\end{array}$ & Lane-miles & $\begin{array}{l}\text { "Initial" Treatment } \\
\text { (Year 2001) }\end{array}$ \\
\hline $\begin{array}{l}\text { Excellent-Good } \\
(\mathrm{IRI}=60-100)\end{array}$ & Trigger $>118$ & 73 & 115 & Do Nothing \\
\hline $\begin{array}{c}\text { Good } \\
\text { (IRI=101 -125) }\end{array}$ & Trigger $>118$ & 112 & 2 & Do Nothing \\
\hline $\begin{array}{c}\text { Good - Fair } \\
(\text { IRI = } 126-150)\end{array}$ & Trigger $>134$ & $\mathrm{n} / \mathrm{a}$ & 0 & $\begin{array}{c}\text { Preventive } \\
\text { Maintenance } \\
\end{array}$ \\
\hline $\begin{array}{c}\text { Fair } \\
(\text { IRI }=151-200) \\
\end{array}$ & Trigger $>151$ & 159 & 6 & $\begin{array}{c}\text { Resurfacing Partial } \\
\text { 3-R Standards }\end{array}$ \\
\hline $\begin{array}{c}\text { Poor } \\
(\text { IRI }=>200)\end{array}$ & Trigger $>200$ & $\mathrm{n} / \mathrm{a}$ & 0 & Reconstruction \\
\hline All Conditions & $\mathrm{n} / \mathrm{a}$ & $\mathrm{n} / \mathrm{a}$ & 124 & $\begin{array}{l}\text { Maintenance } \\
\text { (AAMEX) }\end{array}$ \\
\hline
\end{tabular}

\section{Non-Interstate Non-NHS Pavement Condition and Initial Treatment}

With 19,170 lane-miles, this pavement family represents the largest category in the analysis. Sixty-six percent of pavements in this family were in excellent condition and one percent were in poor condition. Tables 2.28, 2.29 and 2.30 present the initial conditions and initial treatments to HMA, PCC, and COMP pavements respectively. 


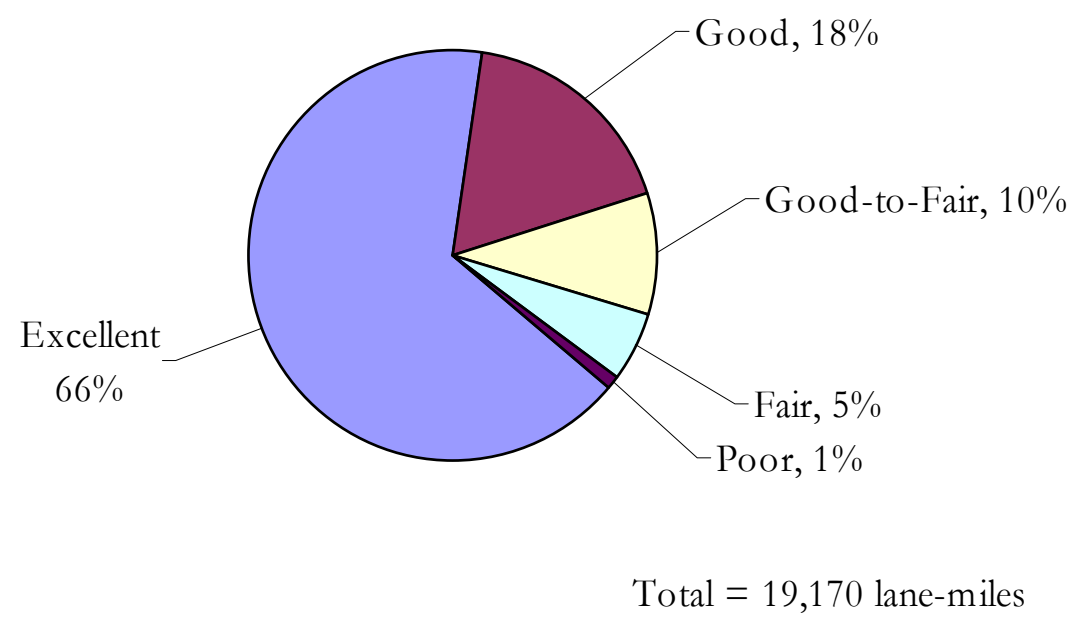

Figure 2.13 Distribution of Non-Interstate Non-NHS by Pavement Condition as of Year 2001

Table 2.28 Non-Interstate, Non-NHS, HMA Pavement Treatment Thresholds

\begin{tabular}{ccccc}
\hline Condition (IRI) & $\begin{array}{c}\text { Deficiency } \\
\text { Level IRI }\end{array}$ & $\begin{array}{c}\text { Existing } \\
\text { Average IRI }\end{array}$ & $\begin{array}{c}\text { Lane- } \\
\text { miles }\end{array}$ & $\begin{array}{c}\text { "Initial" Treatment } \\
\text { (Year 2001) }\end{array}$ \\
\hline $\begin{array}{c}\text { Excellent-Good } \\
\text { (IRI }=60-100)\end{array}$ & Trigger $>118$ & 78 & 12222 & Do Nothing \\
\hline $\begin{array}{c}\text { Good } \\
(\text { IRI }=101-125)\end{array}$ & Trigger $>118$ & 112 & 3218 & Do Nothing \\
\hline $\begin{array}{c}\text { Good }- \text { Fair } \\
\text { IRI }=126-150)\end{array}$ & Trigger $>134$ & 136 & 1771 & Preventive Maintenance \\
\hline $\begin{array}{c}\text { Fair } \\
(\text { IRI }=151-200)\end{array}$ & Trigger $>151$ & 169 & 961 & $\begin{array}{c}\text { Resurfacing Partial 3-R } \\
\text { Standards }\end{array}$ \\
\hline $\begin{array}{c}\text { Poor } \\
\text { (IRI }=>200)\end{array}$ & Trigger $>200$ & 222 & 184 & Reconstruction \\
\hline All Conditions & $\mathrm{n} / \mathrm{a}$ & $\mathrm{n} / \mathrm{a}$ & 18356 & Maintenance (AAMEX) \\
\hline
\end{tabular}


Table 2.29 Non-Interstate Non-NHS PCC Pavement Treatment Thresholds

\begin{tabular}{ccccc}
\hline Condition (IRI) & $\begin{array}{c}\text { Deficiency } \\
\text { Level IRI }\end{array}$ & $\begin{array}{c}\text { Existing } \\
\text { Average IRI }\end{array}$ & $\begin{array}{c}\text { Lane- } \\
\text { miles }\end{array}$ & $\begin{array}{c}\text { "Initial" Treatment } \\
\text { (Year 2001) }\end{array}$ \\
\hline $\begin{array}{c}\text { Excellent-Good } \\
(\text { IRI }=60-100)\end{array}$ & Trigger $>118$ & 79 & 341 & Do Nothing \\
\hline $\begin{array}{c}\text { Good } \\
\text { (IRI }=101-125)\end{array}$ & Trigger $>118$ & 107 & 157 & Do Nothing \\
\hline $\begin{array}{c}\text { Good }- \text { Fair } \\
(\text { IRI }=126-150)\end{array}$ & Trigger $>134$ & 135 & 76 & $\begin{array}{c}\text { Joint and Crack } \\
\text { Sealing }\end{array}$ \\
\hline $\begin{array}{c}\text { Fair } \\
(\text { IRI }=151-200)\end{array}$ & Trigger $>151$ & 170 & 61 & $\begin{array}{c}\text { Resurfacing Partial } \\
\text { 3-R Standards }\end{array}$ \\
\hline $\begin{array}{c}\text { Poor } \\
(\text { IRI }=>200)\end{array}$ & Trigger $>200$ & 214 & 26 & $\begin{array}{c}\text { Reconstruction } \\
\text { All Conditions }\end{array}$ \\
$\mathrm{n} / \mathrm{a}$ & $\mathrm{n} / \mathrm{a}$ & 661 & $\begin{array}{c}\text { Maintenance } \\
\text { (AAMEX) }\end{array}$ \\
\hline
\end{tabular}

Table 2.30 Non-Interstate Non-NHS COMP Pavement Treatment Thresholds

\begin{tabular}{ccccc}
\hline Condition (IRI) & $\begin{array}{c}\text { Deficiency } \\
\text { Level IRI }\end{array}$ & $\begin{array}{c}\text { Existing } \\
\text { Average IRI }\end{array}$ & $\begin{array}{c}\text { Lane- } \\
\text { miles }\end{array}$ & $\begin{array}{c}\text { "Initial" Treatment } \\
\text { (Year 2001) }\end{array}$ \\
\hline $\begin{array}{c}\text { Excellent-Good } \\
\text { (IRI }=60-100)\end{array}$ & Trigger $>118$ & 79 & 128 & Do Nothing \\
\hline $\begin{array}{c}\text { Good } \\
(\text { IRI }=101-125)\end{array}$ & Trigger $>118$ & 103 & 2 & Do Nothing \\
\hline $\begin{array}{c}\text { Good }- \text { Fair } \\
(\text { IRI }=126-150)\end{array}$ & Trigger $>134$ & 131 & 21 & Preventive Maintenance \\
\hline $\begin{array}{c}\text { Fair } \\
(\text { IRI }=151-200)\end{array}$ & Trigger $>151$ & 152 & 1 & $\begin{array}{c}\text { Resurfacing Partial 3-R } \\
\text { Standards }\end{array}$ \\
\hline $\begin{array}{c}\text { Poor } \\
\text { IRI }=>200)\end{array}$ & Trigger $>200$ & $\mathrm{n} / \mathrm{a}$ & 0 & $\begin{array}{c}\text { Reconstruction } \\
\text { All Conditions }\end{array}$ \\
$\mathrm{n} / \mathrm{a}$ & $\mathrm{n} / \mathrm{a}$ & 153 & Maintenance (AAMEX) \\
\hline
\end{tabular}

The methodology used for the treatment of pavements that were initially in poor condition is illustrated in Figure 2.14. Pavements in fair condition follow a similar process, but the analyst compares the initial IRI to 151 and the initial treatment for pavements with IRI >151 would be Resurfacing Partial 3-R Standards. 


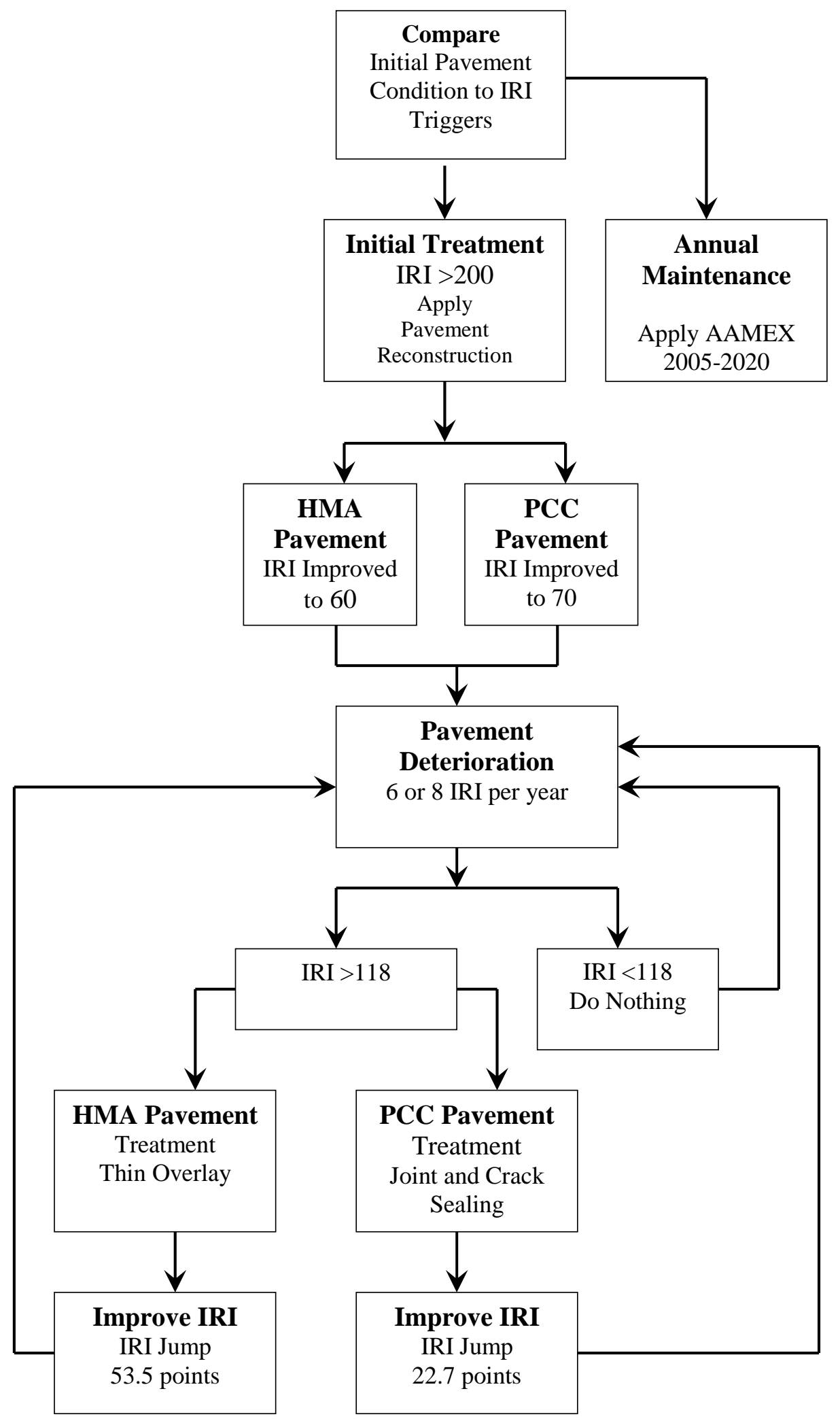

Figure 2.14 Methodology for Poor Pavements 


\section{Project Costs in Manual Condition Analysis}

As previously discussed, the manual condition analysis method utilizes information on average costs per lane-mile for treatments by pavement type as described by Lamptey et al. (2004) and average annual maintenance expenditure (AAMEX) models by Labi and Sinha (2003). The total pavement needs included the costs to maintain the existing pavement as well as to maintain the added pavements associated with capacity improvement projects in INDOT's LRP. Based on information from INDOT's Program Development Division, an additional nine percent of total repair costs has been added to repair project costs for preliminary planning and the cost of design plan preparations. In addition, a contingency of 10 percent of the project costs was added for cost overruns based on information from INDOT’s Program Development Division.

\section{5-year Preservation Needs for Existing Roads}

Scenario A uses a pavement deterioration rate of 8.0 IRI per year, and Scenario B uses a pavement deterioration rate of 6.0 IRI per year. The 15-year pavement preservation need for existing roads under Scenario A for the period 2006-2020 is \$5,544,222,285; while under Scenario B it is \$ 4,938,927,145; as shown in Tables 2.31 and 2.32. The preservation needs discussed in this section do not include any shoulder work or added-capacity projects. The term "repairs only" applies to those costs associated with the "initial" treatments applied in the year 2001. The 15-year need between 2006-2020 was estimated by deducting the expected preservation expenditures during 2002-2005 from the 2002-2020 needs. Data from 2002 and 2003 was used to estimate preservation expenditures during 2002-2005. 
Table 2.31 Fifteen-year Pavement Condition Preservation Needs Scenario A

\begin{tabular}{lcccc}
\hline & Interstate & $\begin{array}{c}\text { Non-Interstate } \\
\text { NHS }\end{array}$ & $\begin{array}{c}\text { Non-Interstate } \\
\text { Non NHS }\end{array}$ & $\begin{array}{c}\text { Total all } \\
\text { Locations }\end{array}$ \\
\cline { 2 - 5 } Lane-Miles of Analysis & 4,143 & 4,814 & 17,972 & 26,929 \\
2002 Initial Repair Costs & $\$ 30,152,561$ & $\$ 77,218,728$ & $\$ 729,036,542$ & $\$ 836,407,831$ \\
2002 AAMEX & $\$ 3,507,973$ & $\$ 6,742,570$ & $\$ 25,451,291$ & $\$ 35,701,834$ \\
Deterioration & $\$ 519,055,202$ & $\$ 672,580,430$ & $\$ 2,917,184,584$ & $\$ 4,108,820,216$ \\
2003-2020 & $\$ 59,635,545$ & $\$ 114,623,682$ & $\$ 432,671,952$ & $\$ 606,931,179$ \\
AAMEX 2003-2020 & $\$ 49,428,699$ & $\$ 67,481,924$ & $\$ 328,159,901$ & $\$ 445,070,524$ \\
$\begin{array}{l}\text { Planning \& Design (9\%) } \\
\text { Repairs Only) }\end{array}$ & $\$ 56,062,154$ & $\$ 81,546,915$ & $\$ 397,305,002$ & $\$ 534,914,072$ \\
Cost Overruns (10\%) \\
(Repairs Only)
\end{tabular}

Table 2.32 Fifteen-year Pavement Condition Preservation Needs Scenario B

\begin{tabular}{lcccc}
\hline & Interstate & $\begin{array}{c}\text { Non-Interstate } \\
\text { NHS }\end{array}$ & $\begin{array}{c}\text { Non-Interstate } \\
\text { Non NHS }\end{array}$ & $\begin{array}{c}\text { Total all } \\
\text { Locations }\end{array}$ \\
\cline { 2 - 5 } Lane-Miles of Analysis & 4,419 & 5,135 & 19,170 & 28,725 \\
2002 Initial Repair Costs & $\$ 30,152,561$ & $\$ 77,218,728$ & $\$ 729,036,542$ & $\$ 836,407,831$ \\
2002 AAMEX & $\$ 3,507,973$ & $\$ 6,742,570$ & $\$ 25,451,291$ & $\$ 35,701,834$ \\
Deterioration 2003-2020 & $\$ 512,200,685$ & $\$ 617,304,103$ & $\$ 2,478,419,895$ & $\$ 3,607,924,683$ \\
AAMEX 2003-2020 & $\$ 57,037,087$ & $\$ 113,467,786$ & $\$ 431,719,453$ & $\$ 602,224,326$ \\
$\begin{array}{l}\text { Planning \& Design (9\%) } \\
\text { Repairs Only) }\end{array}$ & $\$ 48,811,792$ & $\$ 62,507,055$ & $\$ 288,671,079$ & $\$ 399,989,926$ \\
$\begin{array}{l}\text { Cost Overruns (10\%) } \\
\text { Repairs Only) }\end{array}$ & $\$ 55,364,129$ & $\$ 75,527,578$ & $\$ 349,410,208$ & $\$ 480,301,916$ \\
\hline Subtotal 2002-2020 & $\$ 707,074,228$ & $\$ 952,767,820$ & $\$ 4,302,708,468$ & $\$ 5,962,550,516$ \\
\hline Less 2002-2005 Spendinga & & & $\$ 1,023,623,371$ \\
\hline Adjusted Subtotal & & & $\$ 4,938,927,145$ \\
\hline
\end{tabular}




\section{Added Lanes Capacity Needs}

INDOT's Program Development Division provided a database of all LRP projects for use in the present study. Estimation of added-lane capacity improvement needs is based on those identified in the INDOT LRP. The database included information on the project length, the project cost and the number lanes at the start and at the end of the project. The database was sorted to include only those projects planned between 2006 and 2020 and the lane-miles of added-lanes were estimated by taking the product of the project length and the change in the number of lanes. A total of 794 linear miles of added-lanes representing 1,630 lane-miles are planned to be added to the state highway system between 2006 and 2020. Of these 1,630 lane-miles, a total of 760 lane-miles (47\%) are

planned on the interstate system. According to Lamptey et al. (2004), approximately 77 percent of interstate pavements are categorized as composite pavements (HMA layer overlying or underlying a PCC layer) and there has been an increasing trend towards the use of asphalt overlays over existing concrete pavements. For this reason, the present study assumes that added-lanes on the interstate system are PCC. If the current trend continues and they receive an HMA overlay in the future, they will be categorized as composite pavements which is consistent with the predominate interstate pavement type. Lamptey et al. (2004) also states that 64 percent of the entire state highway network is categorized as HMA, and for this reason, added-lanes for non-interstate highways are assumed to be HMA in the present study.

As was previously discussed, all added-lane projects identified in the LRP between 2006 and 2020 were grouped together for a total capital cost (initial construction cost) of 4.68 billion dollars (2002\$). The average annual maintenance expenditure on the new lane-miles of pavement was calculated based on $\$ 1,326$ per lane-mile from previous research (Labi and Sinha 2003). The 15-year cost of preserving added-lanes is discussed in the following two sections. For the needs analysis, added-lanes were maintained in excellent to good condition over the 15-year period, using a trigger of 118 IRI for joint and crack sealing for PCC pavements or thin overlays for HMA pavements. The initial IRI of the added-lanes is assumed to be 70 IRI for PCC pavements (interstate) and 60 IRI for HMA pavements (non-interstate).

\section{5-yr Preservation Needs for Added Lanes}

Two scenarios were used in this analysis: Scenario A (which assumed a pavement deterioration rate of 8 IRI per year) and Scenario B (which assumed a pavement deterioration rate of 6 IRI per year. There were two variations used for these scenarios: Resurfacing Partial 3-R Standards applied after 10 years or after 15 years at a cost of $\$ 297,263$ per lane-mile. Using the deterioration rate of eight IRI per year, it was determined that there was no need for pavement repair treatments such as thin overlays or joint and crack sealing on added lanes to the interstate system until 2012. 
The estimated IRI trends of the new pavements at added-lanes between 2011 and 2020 based on an initial IRI of 70 and a deterioration rate of eight IRI per year are shown in Appendix K.

For Scenario A, the combined cost for the preservation of all added-lanes is $\$ 186,534,221$ (2002\$) or approximately $\$ 12.5$ million per year. The 15 -year cost for the preservation of interstate added-lanes is $\$ 91,098,211$ and $\$ 95,436,010$ for non-interstate added-lanes. These costs include Resurfacing Partial 3-R Standards after 10 years. The total 15-year cost for the preservation of added-lanes for interstate and non-interstate pavements with Resurfacing Partial 3-R Standards after 15 years, rather than after 10 years, is $\$ 65,058,941$ (2002\$) or approximately $\$ 4.4$ million per year. Tables 2.33 and 2.34 summarize the annual costs associated with the preservation of added-lanes on the interstate and non-interstate roads respectively.

Table 2.33 Fifteen-year Preservation Needs for Interstate Added-lanes, Scenario A

\begin{tabular}{|c|c|c|c|c|}
\hline Year & $\begin{array}{l}\text { Added Lane- } \\
\text { miles by Year }\end{array}$ & $\begin{array}{c}\text { Cumulative } \\
\text { Lane-miles } \\
\text { Added }\end{array}$ & $\begin{array}{c}\text { Repair Costs } \\
\text { (Joint and Crack } \\
\text { Seal) }\end{array}$ & $\begin{array}{c}\text { Annual } \\
\text { Maintenance } \\
\text { (AAMEX) }\end{array}$ \\
\hline 2006 & 10.16 & 0 & $\$ 3,036,615$ & $\$ 0$ \\
\hline 2007 & 9.62 & 10.16 & $\$ 2,875,220$ & $\$ 13,473$ \\
\hline 2008 & 67.4 & 19.78 & $\$ 20,144,474$ & $\$ 26,231$ \\
\hline 2009 & 30.4 & 87.18 & $\$ 9,069,551$ & $\$ 115,612$ \\
\hline 2010 & 161.2 & 117.58 & $\$ 48,092,490$ & $\$ 155,926$ \\
\hline 2011 & 10.64 & 278.78 & $\$ 11,468$ & $\$ 369,697$ \\
\hline 2012 & 132.4 & 289.42 & $\$ 71,354$ & $\$ 383,807$ \\
\hline 2013 & 74.02 & 421.82 & $\$ 39,892$ & $\$ 559,386$ \\
\hline 2014 & 75.2 & 495.84 & $\$ 40,528$ & $\$ 657,546$ \\
\hline 2015 & 106 & 571.04 & $\$ 0$ & $\$ 757,271$ \\
\hline 2016 & 11.92 & 677.04 & $\$ 0$ & $\$ 897,840$ \\
\hline 2017 & 14.8 & 688.96 & $\$ 0$ & $\$ 913,647$ \\
\hline 2018 & 0 & 703.76 & $\$ 0$ & $\$ 933,274$ \\
\hline 2019 & 50.04 & 703.76 & $\$ 0$ & $\$ 933,274$ \\
\hline 2020 & 57 & 753.8 & $\$ 0$ & $\$ 999,633$ \\
\hline Subtotal & 810.8 & 753.8 & $\$ 83,381,593$ & $\$ 7,716,618$ \\
\hline \multicolumn{5}{|c|}{$\begin{array}{ll}\text { Total Cost } & \$ 91,098,211\end{array}$} \\
\hline \multirow{2}{*}{\multicolumn{5}{|c|}{ Notes: }} \\
\hline \multirow{2}{*}{\multicolumn{5}{|c|}{ Added-lanes per the INDOT LRP. }} \\
\hline & & & & \\
\hline \multicolumn{5}{|c|}{$\begin{array}{l}\text { Pavement deterioration rate eight IRI per year. } \\
\text { Average annual maintenance cost } \$ 1,326 \text { per lane mile (2002\$) (Labi and Sinha 2003). }\end{array}$} \\
\hline \multicolumn{5}{|c|}{$\begin{array}{l}\text { Average annual maintenance cost } \$ 1,326 \text { per lane mile (2002\$) (Labi and Sinha 2003). } \\
\text { Trigger value for joint and crack sealing treatment, } 118 \text { IRI at a cost of } \$ 539 \text { per lane mile. }\end{array}$} \\
\hline \multicolumn{5}{|c|}{ Resurfacing Partial 3-R Standards applied after 10 years at a cost of $\$ 297,263$. } \\
\hline
\end{tabular}


Table 2.34 Fifteen-year Preservation Needs for Non-Interstate Added-lanes, Scenario A

\begin{tabular}{|c|c|c|c|c|}
\hline Year & $\begin{array}{c}\text { Added } \\
\text { Lane-miles } \\
\text { by Year }\end{array}$ & $\begin{array}{l}\text { Cumulative } \\
\text { Lane-miles } \\
\text { (Added) }\end{array}$ & $\begin{array}{l}\text { Repair Costs } \\
\text { (Thin Overlay) }\end{array}$ & $\begin{array}{c}\text { Annual } \\
\text { Maintenance }\end{array}$ \\
\hline 2006 & 93.7 & 0 & $\$ 41,475,397$ & $\$ 81,252$ \\
\hline 2007 & 70.87 & 93.7 & $\$ 26,218,461$ & $\$ 205,510$ \\
\hline 2008 & 23.4 & 164.57 & $\$ 8,656,865$ & $\$ 299,492$ \\
\hline 2009 & 15.42 & 187.97 & $\$ 5,704,652$ & $\$ 330,524$ \\
\hline 2010 & 3.12 & 203.39 & $\$ 1,154,249$ & $\$ 350,972$ \\
\hline 2011 & 46.06 & 206.51 & $\$ 3,348,048$ & $\$ 355,110$ \\
\hline 2012 & 10.44 & 252.57 & $\$ 758,871$ & $\$ 416,191$ \\
\hline 2013 & 49.58 & 263.01 & $\$ 0$ & $\$ 430,036$ \\
\hline 2014 & 44.69 & 312.59 & $\$ 0$ & $\$ 495,785$ \\
\hline 2015 & 8.34 & 357.28 & $\$ 0$ & $\$ 555,050$ \\
\hline 2016 & 192.2 & 365.62 & $\$ 0$ & $\$ 566,110$ \\
\hline 2017 & 120.4 & 557.82 & $\$ 0$ & $\$ 820,991$ \\
\hline 2018 & 19.86 & 678.22 & $\$ 0$ & $\$ 980,657$ \\
\hline 2019 & 164.24 & 698.08 & $\$ 0$ & $\$ 1,006,993$ \\
\hline 2020 & 4.2 & 862.32 & $\$ 0$ & $\$ 1,224,796$ \\
\hline Subtotal & 866.52 & 866.52 & $\$ 87,316,541$ & $\$ 8,119,469$ \\
\hline Total Cost & & & & $\$ 95,436,010$ \\
\hline \multicolumn{5}{|l|}{ Notes: } \\
\hline \multicolumn{5}{|c|}{ Added-lanes per the INDOT Long Range Plan. } \\
\hline \multicolumn{5}{|c|}{ Non-interstate pavement is assumed to be HMA. } \\
\hline \multicolumn{5}{|c|}{ Pavement deterioration rate eight IRI per year. } \\
\hline \multicolumn{5}{|c|}{ Average annual maintenance cost $\$ 607$ per lane mile (2002\$) (Labi and Sinha 2003). } \\
\hline \multicolumn{5}{|c|}{ Trigger value for thin overlay, 118 IRI at a cost of $\$ 72,689$ per lane mile. } \\
\hline \multicolumn{5}{|c|}{ Resurfacing Partial 3-R Standards applied after 10 years at a cost of $\$ 297,263$. } \\
\hline \multicolumn{5}{|c|}{$\begin{array}{l}\text { Cumulative lane-miles added, is the number of lane-miles at the end of the year. In this study, lane- } \\
\text { miles are not counted in the maintenance lane-miles until the year following the construction year. }\end{array}$} \\
\hline
\end{tabular}

The 15-year preservation need for added-lanes under Scenario B with resurfacing of pavements after 10 years is $\$ 175,308,608$ (2002\$), or 11.7 million dollars per year. The need for interstate pavements is $\$ 90,790,460$ and the need for non-interstate pavements is $\$ 84,518,148$ as shown in Tables 2.35 and 2.36 respectively. The 15-year preservation needs for added-lanes under Scenario B with resurfacing of pavements after 15 years is $\$ 50,147,022$ (2002\$), or 3.4 million dollars per year with interstate pavement need being $\$ 7,424,606$ and non-interstate pavement need being $\$ 42,722,416$ (2002\$). 
Table 2.35 Fifteen-year Preservation Needs for Interstate Added-lanes, Scenario B

\begin{tabular}{|c|c|c|c|c|}
\hline Year & $\begin{array}{c}\text { Added } \\
\text { Lane-miles } \\
\text { by Year }\end{array}$ & $\begin{array}{l}\text { Cumulative } \\
\text { Lane-miles } \\
\text { (Added) }\end{array}$ & $\begin{array}{c}\text { Repair Costs } \\
\text { (Joint Crack and } \\
\text { Seal) }\end{array}$ & $\begin{array}{c}\text { Annual } \\
\text { Maintenance } \\
\text { (AAMEX) }\end{array}$ \\
\hline 2006 & 10.16 & 0 & $\$ 3,031,140$ & $\$ 0$ \\
\hline 2007 & 9.62 & 10.16 & $\$ 2,870,036$ & $\$ 13,473$ \\
\hline 2008 & 67.4 & 19.78 & $\$ 20,108,150$ & $\$ 26,231$ \\
\hline 2009 & 30.4 & 87.18 & $\$ 9,053,168$ & $\$ 115,612$ \\
\hline 2010 & 161.2 & 117.58 & $\$ 48,005,614$ & $\$ 155,926$ \\
\hline 2011 & 10.64 & 278.78 & $\$ 5,734$ & $\$ 369,697$ \\
\hline 2012 & 132.4 & 289.42 & $\$ 0.00$ & $\$ 383,807$ \\
\hline 2013 & 74.02 & 421.82 & $\$ 0.00$ & $\$ 559,386$ \\
\hline 2014 & 75.2 & 495.84 & $\$ 0.00$ & $\$ 657,546$ \\
\hline 2015 & 106 & 571.04 & $\$ 0.00$ & $\$ 757,271$ \\
\hline 2016 & 11.92 & 677.04 & $\$ 0.00$ & $\$ 897,840$ \\
\hline 2017 & 14.8 & 688.96 & $\$ 0.00$ & $\$ 913,647$ \\
\hline 2018 & 0 & 703.76 & $\$ 0.00$ & $\$ 933,274$ \\
\hline 2019 & 50.04 & 703.76 & $\$ 0.00$ & $\$ 933,274$ \\
\hline 2020 & 57 & 753.8 & $\$ 0.00$ & $\$ 999,633$ \\
\hline Subtotal & 810.8 & & $\$ 83,073,842$ & $\$ 7,716,618$ \\
\hline Total Cost & & & & $\$ 90,790,460$ \\
\hline \multicolumn{5}{|l|}{ Notes: } \\
\hline \multicolumn{5}{|c|}{ Added-lanes per the INDOT Long Range Plan. } \\
\hline \multicolumn{5}{|c|}{ Pavement deterioration rate 8 IRI per year. } \\
\hline \multicolumn{5}{|c|}{ Average annual maintenance cost $\$ 1,326$ per lane mile (2002\$) (Labi and Sinha 2003). } \\
\hline \multicolumn{5}{|c|}{ Trigger value for joint and crack sealing treatment, 118 IRI at a cost of $\$ 539$ per lane mile. } \\
\hline \multicolumn{5}{|c|}{ Resurfacing Partial 3-R Standards applied after 10 years at a cost of $\$ 297,263$. } \\
\hline \multicolumn{5}{|c|}{$\begin{array}{l}\text { Cumulative lane-miles added, is the number of lane-miles at the end of the year. In this study, lane- } \\
\text { miles are not counted in the maintenance lane-miles until the year following the construction year. }\end{array}$} \\
\hline
\end{tabular}


Table 2.36 Fifteen-year Preservation Needs for Non-Interstate Added-lanes, Scenario B

\begin{tabular}{|c|c|c|c|c|}
\hline Year & $\begin{array}{l}\text { Added Lane- } \\
\text { miles by Year }\end{array}$ & $\begin{array}{l}\text { Cumulative } \\
\text { Lane-miles } \\
\text { (Added) }\end{array}$ & $\begin{array}{l}\text { Repair Costs } \\
\text { (Thin Overlay) }\end{array}$ & $\begin{array}{c}\text { Annual } \\
\text { Maintenance } \\
\text { (AAMEX) }\end{array}$ \\
\hline 2006 & 93.7 & 0 & $\$ 34,664,453$ & $\$ 81,252$ \\
\hline 2007 & 70.87 & 93.7 & $\$ 26,218,461$ & $\$ 205,510$ \\
\hline 2008 & 23.4 & 164.57 & $\$ 8,656,865$ & $\$ 299,492$ \\
\hline 2009 & 15.42 & 187.97 & $\$ 5,704,652$ & $\$ 330,524$ \\
\hline 2010 & 3.12 & 203.39 & $\$ 1,154,249$ & $\$ 350,972$ \\
\hline 2011 & 46.06 & 206.51 & $\$ 0$ & $\$ 355,110$ \\
\hline 2012 & 10.44 & 252.57 & $\$ 0$ & $\$ 416,191$ \\
\hline 2013 & 49.58 & 263.01 & $\$ 0$ & $\$ 430,036$ \\
\hline 2014 & 44.69 & 312.59 & $\$ 0$ & $\$ 495,785$ \\
\hline 2015 & 8.34 & 357.28 & $\$ 0$ & $\$ 555,050$ \\
\hline 2016 & 192.2 & 365.62 & $\$ 0$ & $\$ 566,110$ \\
\hline 2017 & 120.4 & 557.82 & $\$ 0$ & $\$ 820,991$ \\
\hline 2018 & 19.86 & 678.22 & $\$ 0$ & $\$ 980,657$ \\
\hline 2019 & 164.24 & 698.08 & $\$ 0$ & $\$ 1,006,993$ \\
\hline 2020 & 4.2 & 862.32 & $\$ 0$ & $\$ 1,224,796$ \\
\hline Subtotal & 866.52 & 866.52 & $\$ 76,398,679$ & $\$ 8,119,469$ \\
\hline Total Cost & & & & $\$ 84,518,148$ \\
\hline \multicolumn{5}{|c|}{ Notes: } \\
\hline \multicolumn{5}{|c|}{ Added-lanes per the INDOT Long Range Plan. } \\
\hline \multicolumn{5}{|c|}{ Pavement deterioration rate eight IRI per year. } \\
\hline \multicolumn{5}{|c|}{ Average annual maintenance cost of $\$ 1,326$ per lane mile (2002\$) (Labi and Sinha 2003). } \\
\hline \multirow{2}{*}{\multicolumn{5}{|c|}{$\begin{array}{l}\text { Trigger value for joint and crack sealing treatment, } 118 \text { IRI, at a cost of } \$ 539 \text { per lane mile (2002\$). } \\
\text { Resurfacing Partial 3-R Standards applied after } 10 \text { years at a cost of } \$ 297,263 \text {. }\end{array}$}} \\
\hline & & & & \\
\hline \multicolumn{5}{|c|}{$\begin{array}{l}\text { Cumulative lane-miles added, is the number of lane-miles at the end of the year. In this study, lane-miles } \\
\text { are not counted in the maintenance lane-miles until the year following the construction year. }\end{array}$} \\
\hline
\end{tabular}

\section{5-year Preservation Needs for New Roads}

According to INDOT's LRP, new road construction is defined as a new or relocated roadway, partially or fully on a new alignment. As previously discussed, INDOT's Program Development Division provided a database of all LRP projects for use in the present study. The database included the starting and ending number of lanes for each new road project, the project length and the project cost. The lane-miles of new roads were estimated by taking the product of the project length and the change in the number of lanes provided. The database was sorted for all new roads planned between 2006 and 2020 and the individual project costs were summed together. The analysis of the database determined that new roads accounted for 29.1 percent of the total LRP projects planned between 2006 and 2020 and combined for a total capital improvement cost of 2.9 billion dollars (2002\$). Between 2006 and 2011 all of the new road lane-miles are on non-interstate systems. There were no new roads planned for 2012 or between 2014 and 2016 in the LRP. The 15- 
year preservation needs for these new road construction projects is 4.7 million dollars (2002\$). A summary of the calculations is provided in Table 2.37 .

Table 2.37 Fifteen-year Preservation Needs For New Road Construction

\begin{tabular}{|c|c|c|c|c|c|c|}
\hline Year & $\begin{array}{l}\text { Total } \\
\text { New } \\
\text { Road } \\
\text { Lane- } \\
\text { Miles }\end{array}$ & $\begin{array}{c}\text { Cum. } \\
\text { New } \\
\text { Interstate } \\
\text { Lane- } \\
\text { miles }\end{array}$ & $\begin{array}{l}\text { Cum. New } \\
\text { Non- } \\
\text { Interstate } \\
\text { Lane-miles } \\
\text { Added }\end{array}$ & $\begin{array}{c}\text { Interstate } \\
\text { AAMEX Cost } \\
\text { per year }\end{array}$ & $\begin{array}{c}\text { Non-Interstate } \\
\text { AAMEX Cost } \\
\text { per year }\end{array}$ & Total \\
\hline 2006 & 40.26 & 0.00 & 40.26 & $\$ 0$ & $\$ 50,737$ & $\$ 50,737$ \\
\hline 2007 & 152.00 & 0.00 & 192.26 & $\$ 0$ & $\$ 142,947$ & $\$ 142,947$ \\
\hline 2008 & 64.28 & 0.00 & 256.54 & $\$ 0$ & $\$ 181,942$ & $\$ 181,942$ \\
\hline 2009 & 5.20 & 0.00 & 261.74 & $\$ 0$ & $\$ 185,097$ & $\$ 185,097$ \\
\hline 2010 & 5.84 & 0.00 & 267.58 & $\$ 0$ & $\$ 188,640$ & $\$ 188,640$ \\
\hline 2011 & 83.46 & 0.00 & 351.04 & $\$ 0$ & $\$ 239,270$ & $\$ 239,270$ \\
\hline 2013 & 96.00 & 22.80 & 424.24 & $\$ 30,236$ & $\$ 283,677$ & $\$ 313,912$ \\
\hline 2017 & 86.48 & 100.88 & 432.64 & $\$ 133,780$ & $\$ 288,773$ & $\$ 422,552$ \\
\hline 2018 & 328.00 & 428.88 & 432.64 & $\$ 568,749$ & $\$ 288,773$ & $\$ 857,521$ \\
\hline 2019 & 34.84 & 428.88 & 467.48 & $\$ 568,749$ & $\$ 309,908$ & $\$ 878,657$ \\
\hline 2020 & 271.50 & 700.38 & 467.48 & $\$ 928,792$ & $\$ 309,908$ & $\$ 1,238,700$ \\
\hline Total & 1167.8 & & & $\$ 2,230,304$ & $\$ 2,469,673$ & $\$ 4,699,977$ \\
\hline \multicolumn{7}{|l|}{ Notes: } \\
\hline \multicolumn{7}{|c|}{ Assume new interstate pavement is PCC } \\
\hline \multirow{2}{*}{\multicolumn{7}{|c|}{ AAMEX for Interstates is $\$ 1326$ per lane-mile (2002\$) (Labi and Sinha 2003). }} \\
\hline \multicolumn{4}{|c|}{ AAMEX for Non-Interstates is $\$ 607$ per lane-mile (2002\$) (Labi and Sinha 2003). } & & & \\
\hline \multicolumn{7}{|c|}{ Assume no pavement repair (e.g. Resurfacing Partial 3-R Standards) for the first 15 years. } \\
\hline \multicolumn{7}{|c|}{$\begin{array}{l}\text { Cumulative lane-miles added, is the number of lane-miles at the end of the year. In this study, lane-miles are not counted } \\
\text { in the maintenance lane-miles until the year following the construction year. }\end{array}$} \\
\hline
\end{tabular}

\section{Safety Improvement Needs}

Lamptey (2004) investigated the state highway safety needs utilizing the Indiana Safety Management System software package (SMSS), which utilizes a systematic process to consider physical road deficiencies. The analysis was performed without imposing any budgetary constraints and considered physical road deficiencies without regard for traffic volumes or crash histories. A comprehensive road safety database relating the historical crash counts to the roadway characteristics was developed by Lamptey (2004) from a number of separate datasets for the road sections on the Indiana state highway network, including: The Roadway Inventory Dataset, The Route Alignment Dataset, Indiana Crash Dataset, and the Road Sections Dataset. The four datasets were merged into one comprehensive database in order to model the relation between the crash occurrences on a road as a function of its geometric characteristics. The four datasets are described later in this section. 
The analysis determined that the safety needs for state controlled roads was 10.3 million per year, in year 2005 constant dollars. The estimate was converted to year 2002 constant dollars for the present study (9.4 million dollars), yielding a 15-year need of approximately 141.6 million dollars for state highway sections. Safety needs at intersections were not included in the Lamptey (2004) analysis. Therefore, these needs were derived from safety improvements identified in the LRP. All projects identified as Transportation System Management (TSM) projects in the LRP between 2006 and 2020 were classified as safety needs for the purpose of this study. This categorization was made in consultation with INDOT's Program Development Division. TSM projects are directly related to traffic operations improvements, which can improve safety, and can include minor intersection improvements such as adding a left-turn bay. The INDOT LRP database was sorted for all TSM projects between 2006 and 2020. The project costs from the LRP were summarized for a 15-year need of $\$ 60,749,063$ in year 2002 constant dollars. Therefore, the total safety need between 2006 and 2020 based on Lamptey (2004) and the sorted LRP database is approximately 194 million dollars in year 2002 constant dollars.

\section{Estimation of Bridge Needs}

The bridge needs analysis in the present study builds upon the work by Rodriguez (2004) who evaluated the physical and monetary bridge preservation needs for the 10 -year period between 2006 and 2015 and also updated cost and deterioration models with emphasis on deck deterioration models for concrete and steel bridges. Cost models were developed for various combinations of rehabilitation and replacement activities for steel and concrete bridge elements. Three alternative approaches were used in the Rodriguez bridge needs assessment: an age-based approach utilizing fixed time intervals for bridge replacement and bridge rehabilitation; a deck deterioration-based approach utilizing trigger values for deck condition ratings and the Indiana Bridge Management System (IBMS) software package approach that uses trigger values for determining element condition ratings as well as load capacity and functional characteristics.

Rodriguez (2004) found that the IBMS approach considered all aspects of a bridge, not simply its age or deck deterioration. Information such as road class and established minimum standards that vary by element, deck geometry, structural evaluation, inventory rating, and vertical clearance were all included in the IBMS approach to assign a preservation action. Consequently, even though the IBMS approach yielded lower estimates than those from age-based or deck deterioration-based approaches, this approach was used in the present study to determine the physical needs of the bridge infrastructure. 


\section{IBMS Software}

The IBMS software is a decision tool to help bridge engineers monitor existing bridge conditions and plan, program and manage bridge improvements. The IBMS software provides results on the condition of the overall network. IBMS uses the Indiana State Bridge Inventory (ISBI), a database that includes information from regular field inspections and historical bridge information. Example data items are the highway route, bridge number, county code, year built, year reconstructed, total deck width, and deck condition. Deterioration models predict the future condition of bridge elements and the IBMS project selection module selects bridge projects using four independent sub-modules: Decision Tree Module (DTREE), Life-cycle Cost Analysis Module (COST), Ranking Module (RANK), and Optimization Module (OPT) (Gion et al., 1993).

In the DTREE Module, appropriate repair, rehabilitation, reconstruction, and replacement activities are selected based on bridge structural condition rating, physical characteristics, traffic information, and highway type. The DTREE module also estimates the repair year and project cost. The COST module performs a life-cycle cost analysis that establishes the economic consequences of implementing a particular project. The RANK module prioritizes projects, and the OPT module determines the best use of funds by investigating possible combinations of projects.

\section{Status of Indiana State Highway Bridges}

Indiana state highway system bridges are located on the National Highway System (NHS) and other roads. There are 2,174 bridges on the NHS, 993 bridges on the Major State Transportation Plan (STP) roads, and 1,579 are on Minor STP roads (Rodriguez, 2004). A total of 269 local bridges are the responsibility of INDOT. As the NHS and Major STP bridges carry higher volumes of traffic, they also generally deteriorate at higher rates when compared to Local and Minor STP bridges. Rodriguez (2004) determined that over 11\% of the Indiana state highway bridges are older than 70 years. Assuming a 70 -year design life for concrete bridges and given current loading and weather conditions, $8 \%$ of state highway bridges appear to have been due for replacement in 1983 while approximately 35\% and 20\% will be due for replacement in 2013 and 2040 respectively. The primary age of a bridge was defined by Rodriguez (2004) as the number of years since the bridge construction as of the year of the study (2003). Secondary age was defined as the number of years since the last major rehabilitation or widening as of 2003.

Rodriguez (2004) determined that only 3\% of steel bridges are older than 70 years; $13 \%$

(over 300) were 47 years old, which means that these bridges will be due for replacement in 23 years or 2026, when they will reach the end of their design lives. 


\section{Data Collection and Database Development}

The bridge inventory input file from INDOT was used in the present study. The bridge inventory file contains information on 5,561 bridges on the state system. There is a complete list in Appendix $G$ of the data items provided for bridges in the IBMS input file. The data file containing the list of bridges identified as needing repair or replacement between 2006 and 2015 was based on Rodriguez (2004) as well and contained 1,280 bridges.

Bridge physical needs and costs during 2006-2015 were obtained from Rodriguez (2004). The IBMS software was run to determine needs for the additional years to estimate total bridge needs for 2006-2020. All unit costs were taken from Rodriguez (2004), who revised the IBMS cost models in instances where there was enough historical data to do so.

\section{Bridge Needs Analysis and Results}

Bridge preservation activities may generally be described as those that extend bridge life. The Bridge Project Coding System (BPCS) at INDOT identifies two major categories of preservation activities at the engineering level: bridge replacement and bridge rehabilitation or repair. Bridge rehabilitation or repair includes subcategories such as: bridge deck reconstruction, bridge widening, bridge painting, bridge maintenance and repair, raising bridge/lowering pavement, superstructure replacement, substructure repair and rehabilitation and bridge channel corrections (Rodriguez, 2004). INDOT bridge maintenance and repair activities include repairing or replacing joints, straightening beams, rail replacement, removal and replacement of beams and bridge cleaning.

A total of 125 bridges were identified for bridge preservation needs between 2016 and 2020. Since 1,280 bridges were identified for bridge preservation needs between 2006 and 2015 by Rodriguez (2004), the total number of bridges needing rehabilitation or replacement was 1,405 during the 15-year horizon of 2006-2020.

IBMS defines 50 alternative bridge preservation activities, a full description of which is provided in Table G.2 in Appendix G. Rodriguez (2004) sorted the IBMS work codes into aggregate work categories in order to categorize the work either as a rehabilitation or replacement activity. The resulting work groups are listed in Table 2.38. Bridge rehabilitation includes all categories except bridge replacement. Unit costs used in IBMS for various work categories were updated by Rodriguez (2004). 
Table 2.38 Bridge Work Activity Groups

\begin{tabular}{|c|c|c|}
\hline Activity Group & $\begin{array}{l}\text { IBMS } \\
\text { Code }\end{array}$ & Work Type \\
\hline Deck Rehab & $\begin{array}{c}1 \\
5 \\
21 \\
24 \\
26 \\
31 \\
34 \\
41\end{array}$ & $\begin{array}{l}\text { Deck Rehab } \\
\text { Deck \& Super Rehab } \\
\text { Deck \& Sub Rehab } \\
\text { Deck \& Super \& Sub Rehab } \\
\text { Widen \& Replace Deck + Super \& Sub Rehab } \\
\text { Deck Rehab + Raise / Lower } \\
\text { Deck \& Super Rehab + Raise / Lower } \\
\text { Deck \& Super Rehab + Raise / Lower }\end{array}$ \\
\hline Deck Replacement & $\begin{array}{c}3 \\
4 \\
7 \\
8 \\
23 \\
32 \\
33 \\
35 \\
36 \\
43 \\
45 \\
46\end{array}$ & $\begin{array}{l}\text { Deck Replace } \\
\text { Deck Replace + Widen Deck } \\
\text { Deck Replace + Super Rehab } \\
\text { Deck Replace + Super Rehab + Widen Deck } \\
\text { Widen \& Replace Deck + Sub Rehab } \\
\text { Deck Replace + Raise / Lower } \\
\text { Widen \& Replace Deck + Raise / Lower } \\
\text { Deck Replacement + Super Rehab + R/L } \\
\text { Widen \& Replace Deck + Super Rehab + R/L } \\
\text { Widen \& Replace Deck + Sub Rehab + R/L } \\
\text { Deck Replace + Super \& Sub Rehab + R/L } \\
\text { Widen \& Replace Deck + Sup \& Sub Rehab + R/L }\end{array}$ \\
\hline Bridge Replacement & $\begin{array}{l}14 \\
15\end{array}$ & $\begin{array}{l}\text { Bridge Replacement } \\
\text { Widen Deck + Bridge Replacement }\end{array}$ \\
\hline $\begin{array}{l}\text { Strengthen } \\
\text { Superstructure }\end{array}$ & $\begin{array}{l}12 \\
13 \\
30 \\
39 \\
40\end{array}$ & $\begin{array}{l}\text { Strengthen Super } \\
\text { Strengthen Super + Widen Deck } \\
\text { Widen Deck + Strengthen Super + Sub Rehab } \\
\text { Strengthen Super + Raise / Lower } \\
\text { Widen Deck + Strengthen Super + R/L }\end{array}$ \\
\hline $\begin{array}{l}\text { Superstructure } \\
\text { Replacement }\end{array}$ & $\begin{array}{l}10 \\
11\end{array}$ & $\begin{array}{l}\text { Super Replace } \\
\text { Super Replace + Widen Deck }\end{array}$ \\
\hline Other & $\begin{array}{l}16 \\
19\end{array}$ & $\begin{array}{l}\text { Sub Rehab } \\
\text { Culvert Replacement }\end{array}$ \\
\hline
\end{tabular}

Rodriguez (2004) 


\section{Bridge Rehabilitation}

During 2006-2015, 935 bridges were identified for rehabilitation for a total cost of $\$ 449,365,000$ while 82 bridges were identified for bridge rehabilitation between 2016 and 2020 with a total cost of $\$ 93,648,211$. Over the entire 15 -year period, the bridge rehabilitation need would be about $\$ 543$ million or about $\$ 36$ million per year.

\section{Bridge Replacement Needs}

During 2006-2015, 345 bridges were identified for replacement for total cost of $\$ 432,966,000$. Forty-three bridges were identified to be replaced between 2016 and 2020 with a total cost of $\$ 299,440,700$. Over the 15 -year period, the total bridge replacement cost would be $\$ 733$ million or $\$ 48.9$ million per year.

\section{Bridge Preservation Needs}

Table 2.39 presents a summary of preservation needs of existing bridges. About $28 \%$ of the existing bridges would require preservation during the 15-year period, most of which would need rehabilitation. The total fiscal need would be about $\$ 1.275$ billion in 2002 dollars. Details of expected activities are presented in Table 2.40.

Table 2.39 Summary of Bridge Needs Assessment 2006-2020

\begin{tabular}{ccccc}
\hline Work Type & $\begin{array}{c}\text { No. of } \\
\text { Bridges }\end{array}$ & $\begin{array}{c}\text { Percentage of } \\
\text { System }\end{array}$ & $\begin{array}{c}\text { Needs } \\
(\$ Y e a r 2002)\end{array}$ & $\begin{array}{c}\text { Percentage } \\
\text { of Costs }\end{array}$ \\
\hline Bridge Replacement & 388 & $7.6 \%$ & $\$ 732,406,700$ & $57 \%$ \\
Rehabilitation & 1017 & $19.9 \%$ & $\$ 543,013,211$ & $43 \%$ \\
Total & 1405 & $27.5 \%$ & $\$ 1,275,419,911$ & $100 \%$ \\
\hline Total of system & 5118 & & & \\
\hline
\end{tabular}


Table 2.40 Bridge Needs Assessment 2006-2020

\begin{tabular}{|c|c|c|c|}
\hline $\begin{array}{l}\text { IBMS } \\
\text { Code }\end{array}$ & Activity Type & $\begin{array}{c}\text { No. } \\
\text { Bridges }\end{array}$ & $\begin{array}{c}\text { Total Cost } \\
(\$ 1,000)\end{array}$ \\
\hline 1 & Deck Rehabilitation & 635 & $\$ 287,770$ \\
\hline 3 & Deck Replacement & 7 & $\$ 4,364$ \\
\hline 4 & Deck Replacement + Deck Widening & 71 & $\$ 48,019$ \\
\hline 5 & Deck + Superstructure Rehab & 15 & $\$ 8,846$ \\
\hline 8 & Deck Replace +Superstructure Rehab + Deck Widening & 14 & $\$ 24,523$ \\
\hline 10 & Replace Superstructure & 12 & $\$ 18,071$ \\
\hline 11 & Replace Superstructure + Deck Widening & 11 & $\$ 15,446$ \\
\hline 14 & Bridge Replacement & 122 & $\$ 103,558$ \\
\hline 15 & Bridge Replacement + Deck Widening & 266 & $\$ 628,849$ \\
\hline 16 & Substructure Rehab & 11 & $\$ 1,173$ \\
\hline 21 & Deck + Sub Rehab & 2 & $\$ 751$ \\
\hline 23 & Widen + Replace Deck + Sub Rehab & 11 & $\$ 15,188$ \\
\hline 26 & Widen \& Replace Deck + Super + Sub Rehab & 4 & $\$ 5,002$ \\
\hline 28 & Widen Deck + Super Replace + Sub Rehab & 1 & $\$ 2,788$ \\
\hline 31 & Deck Rehab \& Raise/Lower (R/L) & 151 & $\$ 62,718$ \\
\hline 32 & Deck Replace + Raise / Lower & 2 & $\$ 770$ \\
\hline 33 & Widen \& Replace Deck + R/L & 42 & $\$ 29,296$ \\
\hline 34 & Deck +Super Rehab+ R/L & 2 & $\$ 1,562$ \\
\hline 36 & Widen \& Replace Deck + Super Rehab \& R/L & 5 & $\$ 5,103$ \\
\hline 38 & Widen Deck + Super Replacement + R/L & 7 & $\$ 4,838$ \\
\hline 41 & Deck + Sub Rehab + R/L & 1 & $\$ 178$ \\
\hline 43 & Widen \& Replace Deck + Sub Rehab+ R/L & 2 & $\$ 3,854$ \\
\hline \multirow[t]{2}{*}{46} & Widen \& Replace Deck + Sup \& Sub Rehab + R/L & 1 & $\$ 2,928$ \\
\hline & $\begin{array}{ll}1 & \text { Total } \\
\end{array}$ & 1405 & $\$ 1,275,420$ \\
\hline
\end{tabular}

Costs are expressed in Year 2002 constant dollars.

\section{Bridge Preservation Needs for New Bridges}

According to the INDOT LRP, there are two new bridges planned for 2006, two planned for 2013 and one planned for 2016. These planned bridges are expected to require preservation needs during the time horizon of the present study. While the only two bridges planned for 2006 can be expected to need major repair, other three bridges would require some levels of preservation during the period of the study. The 15-year bridge rehabilitation need for existing bridges was found to be $\$ 543$ million (\$Year 2002) with an average cost of $\$ 0.53$ million per bridge. Based on this estimated cost per bridge, the 15-year preservation need for new bridges is $\$ 2.7$ million (\$Year 2002).

\section{Bridge Needs Summary and Conclusions}

Based on the findings of Rodriguez (2004) and the present study, it is estimated that the total bridge preservation cost between 2006 and 2020 is approximately $\$ 1.3$ billion dollars ( $\$$ Year 2002). 


\section{Operating and Other Costs}

Approximately 60 percent of INDOT expenditure between 1995 and 2003 were for construction and 40 percent were non-construction costs as shown in Table 2.41. The largest nonconstruction expenditure was operating cost, which include, among other items, salaries and wages. Program support cost includes such items as computer technical support. Consultant costs represent expenditures for the preparation of plans and studies. "Road leases" are debt service costs or fees paid by INDOT to the Indiana Transportation Finance Authority (ITFA) for bonds that finance highway infrastructure. Indiana law does not allow INDOT to contract debt, so ITFA issues bonds to cover the cost of infrastructure projects and owns the infrastructure until INDOT lease payments have paid for the bond in full. Costs such as maintenance, right of way and consultants are included in needs assessed earlier, but not the overhead costs. Debt service costs are not considered, because bond as a source of financing is not included in the revenue computation. For the present study, overhead costs therefore consist of operating and program support costs. During 1995-2003 overhead was $22 \%$ of the total expenditures. Excluding road leases from the total, expected 15-year financial needs for the state highway system were thus adjusted to reflect overhead needs.

Table 2.41 State Highway Infrastructure Expenditures 1995-2003

\begin{tabular}{|c|c|c|c|c|c|c|c|c|c|}
\hline \multirow{2}{*}{$\begin{array}{l}\text { Non- } \\
\text { Construction } \\
\text { Expenditure }\end{array}$} & \multicolumn{9}{|c|}{ Non-Construction Uses by Year Current\$ (Millions) } \\
\hline & 1995 & 1996 & 1997 & 1998 & 1999 & 2000 & 2001 & 2002 & 2003 \\
\hline Operating & 174.1 & 180.4 & 183.2 & 188.1 & 202.2 & 203.6 & 216.7 & 221.4 & 236.0 \\
\hline $\begin{array}{l}\text { Program } \\
\text { Support }\end{array}$ & 25.0 & 31.6 & 29.0 & 36.9 & 30.9 & 36.4 & 44.1 & 46.7 & 44.5 \\
\hline $\begin{array}{l}\text { Maintenance } \\
\text { Work Program }\end{array}$ & 48.4 & 53.3 & 54.8 & 56.4 & 59.1 & 61.8 & 63.4 & 60.8 & 72.1 \\
\hline Right-of Way & 18.9 & 21.1 & 27.3 & 20.0 & 35.4 & 51.4 & 48.8 & 51.6 & 51.8 \\
\hline $\begin{array}{l}\text { Project } \\
\text { Consultants } \\
\end{array}$ & 19.6 & 26.8 & 24.1 & 42.9 & 53.0 & 70.1 & 56.2 & 58.7 & 59.5 \\
\hline Road Leases & 28.4 & 27.2 & 25.5 & 26.6 & 30.7 & 33.6 & 40.1 & 45.6 & 55.0 \\
\hline $\begin{array}{l}\text { Total Non- } \\
\text { Construction }\end{array}$ & 314.4 & 340.4 & 343.9 & 370.9 & 411.3 & 456.9 & 469.3 & 484.8 & 519.1 \\
\hline Construction & \multicolumn{9}{|c|}{ Construction Uses by Year Current\$ (Millions) } \\
\hline Expenditure & 1995 & 1996 & 1997 & 1998 & 1999 & 2000 & 2001 & 2002 & 2003 \\
\hline $\begin{array}{l}\text { Road \& Bridge } \\
\text { Construction }\end{array}$ & 413.9 & 516.1 & 523.8 & 609.4 & 689.1 & 681.1 & 796.9 & 647.0 & 754.5 \\
\hline Total all uses & 728.3 & 856.5 & 867.8 & 980.3 & $1,100.4$ & $1,138.0$ & $1,266.2$ & $1,131.8$ & $1,273.5$ \\
\hline
\end{tabular}




\section{State Highway Needs Summary and Conclusion}

The preceding sections estimated pavement, bridge, capacity, and safety needs for the 15year horizon between 2006 and 2020. Overhead costs were estimated based on historical trends. Pavement needs were estimated using HERS-ST software and a manual method. Costs for new highway infrastructure, such as new bridges and new roads, were obtained from the LRP. The bridge preservation needs were based on IBMS estimates. Safety needs on through roads were based on estimates by Lamptey (2004), which utilized the Indiana Safety and Congestion Management System Software. Safety improvement needs at intersections were based on improvements identified in the LRP. The total state highway financial need based on HERS-ST analysis is approximately $\$ 29.8$ to $\$ 30.2$ billion (2002\$) as illustrated in Table 2.42. Table 2.43 gives the financing needs resulting from the manual method. The manual method used a network condition based procedure to estimate pavement preservation needs. Other need estimates in the manual method were mainly the same as the HERS-ST method. However, the manual method could not consider shoulder work or alignment improvements. The resulting need estimates were thus substantially lower than those obtained from HERS-ST method. These estimates can therefore be considered as minimum needs primarily to preserve the state highway infrastructure without most safety and capacity improvements. 
Table 2.42 Fifteen-year State Highway Needs, HERS-ST Method

\begin{tabular}{|c|c|}
\hline & Cost (1000s) (\$Year 2002) \\
\hline Pavement, Capacity, Safety (HERS-ST) & $\$ 17,097,504$ to $\$ 17,362,571$ \\
\hline Safety Needs ${ }^{a}$ (Additional) & $\$ 60,749$ \\
\hline $\begin{array}{l}\text { Capacity Needs (LRP) } \\
\text { Interchanges (All) } \\
\text { New Roads } \\
\text { New Bridges } \\
\text { Median Construction } \\
\quad \text { Subtotal Capacity }\end{array}$ & $\begin{array}{l}\$ 1,348,846 \\
\$ 2,896,568 \\
\$ 382,336 \\
\$ 132,085 \\
\$ 4,849,759\end{array}$ \\
\hline $\begin{array}{l}\text { Preservation of New Pavement } \\
\text { Added Lanes } \\
\text { New Roads } \\
\text { Subtotal Preservation of New Pavements }\end{array}$ & $\begin{array}{l}\$ 69,396 \text { to } \$ 186,534 \\
\$ 4,699 \\
\$ 74,095 \text { to } \$ 191,233\end{array}$ \\
\hline $\begin{array}{l}\text { Bridge Needs } \\
\text { IBMS Bridge 2006-2020 Needs } \\
\text { Preservation of Five New Bridges } \\
\text { Subtotal Bridge Needs }\end{array}$ & $\begin{array}{l}\$ 1,275,420 \\
\$ 2,700 \\
\$ 1,278,120\end{array}$ \\
\hline Subtotal 15-year Infrastructure Needs & $\$ 23,270,303$ to $\$ 23,591,579$ \\
\hline Overhead Costs & $\$ 6,563,419$ to $\$ 6,654,035$ \\
\hline Total 15-year State Highway Needs & $\$ 29,833,722$ to $\$ 30,245,614$ \\
\hline
\end{tabular}


Table 2.43 Fifteen-year State Highway Needs, Manual Analysis Method

\begin{tabular}{|c|c|}
\hline & Cost (1000s) (\$Year 2002) \\
\hline Pavement Repair and Maintenance & $\$ 4,976,758$ to $\$ 5,544,222^{a}$ \\
\hline \multicolumn{2}{|l|}{ Safety Needs } \\
\hline Intersection Improvements b & $\$ 60,749$ \\
\hline Road Segment Improvements c & $\$ 132,738$ \\
\hline Subtotal Safety & $\$ 193,487$ \\
\hline \multicolumn{2}{|l|}{ Capacity Needs (LRP)d } \\
\hline Added Travel Lanes & $\$ 4,676,002$ \\
\hline Interchanges (All) & $\$ 1,348,846$ \\
\hline New Roads & $\$ 2,896,568$ \\
\hline New Bridges & $\$ 382,336$ \\
\hline Median Construction & $\$ 132,085$ \\
\hline Subtotal Capacity & $\$ 9,435,837$ \\
\hline Preservation of New Pavement & $\$ 74,095$ to $\$ 191,233$ \\
\hline Bridge Needs & $\$ 1,278,120$ \\
\hline Subtotal 15-year Infrastructure Needs & $\$ 14,647,842$ to $\$ 15,332,444$ \\
\hline Overhead Costs & $\$ 4,131,443$ to $\$ 4,324,535$ \\
\hline Total 15-year State Infrastructure Needs & $\$ 18,779,285$ to $\$ 19,656,979$ \\
\hline $\begin{array}{l}\text { a. Work excludes roadway alignment and shoulder impr } \\
\text { b. INDOT Long Range Plan “TSM" Projects. } \\
\text { c. Cost based on } 10.3 \text { million dollars per year (2005\$), f } \\
\text { d. INDOT Long Range Plan. }\end{array}$ & $\begin{array}{l}\text { vements. } \\
\text { m Lamptey (2004). }\end{array}$ \\
\hline
\end{tabular}

\subsection{Estimation of Needs for Local Highway Network}

The methodology adopted in the present study was based on trigger values to determine the backlog or immediate needs to upgrade the system to an acceptable level of service. Time strategies were used to determine the future preservation and maintenance needs from 2006 to 2020. Table 2.44 illustrates the methods used to determine the pavement and bridge needs. 
Table 2.44: Methods Used for Local System Needs Assessment

\begin{tabular}{|c|c|c|c|}
\hline \multirow{2}{*}{ Roads } & Paved & Backlog & Future Needs \\
\hline & & Trigger Values & $\begin{array}{c}\text { Time Based } \\
\text { Strategies } \\
\text { (Overlays and } \\
\text { Resurfacing at } \\
\text { Intervals) }\end{array}$ \\
\cline { 3 - 5 } & Unpaved & Annual Average Maintenance Expenditure \\
\hline \multirow{2}{*}{ Bridges } & Trigger Values & $\begin{array}{c}\text { Based on Age } \\
\text { (Replacement and } \\
\text { Rehabilitation at } \\
\text { Intervals) }\end{array}$ \\
\hline
\end{tabular}

\subsubsection{Local Roads Inventory}

Locally controlled roads account for 81,350 miles, which represent $90 \%$ of the public roads in the State of Indiana. According to a road condition survey for approximately 3,200 miles of county roads in 2000 and 2001 (LTAP 2001), $53 \%$ of the county roads were less than the recommended minimum width of 18 feet. The same survey also showed that $49 \%$ of the county roads have roughness values greater than 200 inches/mile. Pavements in this category are considered to be in "poor" condition according to the state standards. Paved and unpaved roads are treated separately while estimating the cost associated with the maintenance of local roads and streets in the State of Indiana. Average unit costs per mile required to maintain each type of road can be obtained using different methods, and can be applied to the total mileage. The classification of county, city and town roads by surface type is shown on Table 2.45. It can be noted that unpaved roads represent $35.5 \%$ of the total statewide local highway mileage. 


\begin{tabular}{|c|c|c|c|c|c|c|}
\hline \multicolumn{7}{|c|}{ Table 2.45: Classification of Local Roads by Surface Type } \\
\hline & & $\begin{array}{l}\text { City Route } \\
\text { Length } \\
\text { (miles) }\end{array}$ & $\begin{array}{l}\text { County } \\
\text { Route } \\
\text { Length } \\
\text { (miles) }\end{array}$ & $\begin{array}{l}\text { Town } \\
\text { Route } \\
\text { Length } \\
\text { (miles) }\end{array}$ & $\begin{array}{c}\text { Unknown } \\
\text { Route } \\
\text { Length } \\
\text { (miles) }\end{array}$ & $\begin{array}{l}\text { Total } \\
\text { Route } \\
\text { Length } \\
\text { (miles) }\end{array}$ \\
\hline & Unknown & 93.59 & 242.86 & 46.66 & 0.6 & 383.71 \\
\hline \multirow{4}{*}{$\begin{array}{c}\text { Unpaved } \\
\text { Roads }\end{array}$} & $\begin{array}{c}\text { Unimproved } \\
\text { Road }\end{array}$ & 9.18 & 75.55 & 3.47 & 0 & 88.2 \\
\hline & $\begin{array}{l}\text { Graded and } \\
\text { Drained } \\
\text { Earth Road }\end{array}$ & 15.24 & 486.76 & 7.94 & 0 & 509.94 \\
\hline & $\begin{array}{c}\text { Gravel or } \\
\text { stone Road }\end{array}$ & 360.45 & $27,626.13$ & 289.16 & 0 & $28,275.74$ \\
\hline & $\begin{array}{c}\text { Total } \\
\text { Unpaved }\end{array}$ & 384.87 & $28,188.44$ & 300.57 & 0 & $28,873.88$ \\
\hline \multirow{7}{*}{$\begin{array}{l}\text { Paved } \\
\text { Roads }\end{array}$} & $\begin{array}{c}\text { Bit. Surface- } \\
\text { Treated Road } \\
\text { (<1 inch) }\end{array}$ & 834.82 & $6,600.57$ & 511.95 & 0 & $7,947.34$ \\
\hline & $\begin{array}{l}\text { Mixed Bit. } \\
\text { Road } \\
(<7 \text { inch })\end{array}$ & $6,166.04$ & $28,840.28$ & $3,040.62$ & 8.77 & $38,055.71$ \\
\hline & $\begin{array}{l}\text { Mixed Bit. } \\
\text { Road } \\
(>7 \text { inch })\end{array}$ & 907.39 & $1,030.13$ & 131.27 & 0.43 & $2,069.22$ \\
\hline & $\begin{array}{c}\text { Bit. Concrete, } \\
\text { Sheet or Rock } \\
\text { Asphalt }\end{array}$ & 489.52 & 719.42 & 99.8 & & $1,308.74$ \\
\hline & $\begin{array}{c}\text { Portland } \\
\text { Cement } \\
\text { Concrete } \\
\text { Road }\end{array}$ & $1,363.04$ & 962.30 & 187.76 & & $2,513.10$ \\
\hline & Brick Road & 162.80 & 14.68 & 21.54 & & 199.02 \\
\hline & Total Paved & 9923.61 & $38,167.38$ & 3992.94 & 9.2 & $52,093.13$ \\
\hline \multicolumn{2}{|r|}{ Total Mileage } & $10,402.07$ & $66,598.68$ & $4,340.17$ & 9.80 & $81,350.72$ \\
\hline
\end{tabular}

Source: LTAP (2000a) 


\subsubsection{Approach Using Need/Disbursements Ratio and Time-Based Strategies}

Main objective of the ratio approach is to find a relationship between the estimated needs and the disbursements for a sample of eight counties for two different years 1998 and 2000, and derive an average ratio which could be applied to the projected disbursements over the study period for the entire state. Physical needs are thus determined for the eight counties and compared to the disbursements provided in the annual reports of these counties in 1998 and 2000.

\section{Estimation of Needs for Sample Counties}

\section{Unpaved Roads}

To estimate the needs associated with the maintenance of unpaved roads, a study done at Purdue University (Riverson 1983) was used. The study considered all the costs necessary for the preservation and maintenance of unpaved roads in 3 different counties (Bartholomew, Huntington and Jasper) and derived an average annual cost per mile for the maintenance of unpaved roads. In the present study, values from these three counties were aggregated to a single average value, which was used as representative of the unit cost per mile at a state level for unpaved road preservation and maintenance. The observed values are shown in Table 2.46 .

Table 2.46: Unit Costs Per Mile for Unpaved Roads Maintenance in Year 1983

\begin{tabular}{|c|c|c|c|c|}
\hline \multirow{2}{*}{ County } & \multirow{2}{*}{$\begin{array}{c}\text { Unit Cost } \\
\text { (per mile) - } \\
1983\end{array}$} & \multicolumn{3}{|c|}{ Average Unit Costs (per mile) } \\
\hline & & 1983 & 1998 & 2000 \\
\hline Bartholomew & $\$ 259$ & \multirow{3}{*}{$\$ 603$} & \multirow{3}{*}{$\$ 873.52$} & \multirow{3}{*}{$\$ 1,002.25$} \\
\hline Huntington & $\$ 750$ & & & \\
\hline Jasper & $\$ 800$ & & & \\
\hline
\end{tabular}

\section{Source: (Riverson 1983)}

In the present analysis, the objective was to determine the needs for the preservation and maintenance of unpaved roads in eight counties. Annual county operational reports provide the mileage of unpaved roads in each of these counties. Using the average cost from Table 2.46, the financial needs for the unpaved system preservation and maintenance for each county were estimated for 1998 and 2000 as shown in Table 2.47. 
Table 2.47: Estimated Needs for Preservation and Maintenance of Unpaved Roads in Sample Counties in 1998 and $2000(\$ 1000)$

\begin{tabular}{|c|c|c|c|c|}
\hline County & $\begin{array}{c}\text { Unpaved } \\
\text { Mileage in } \\
1998\end{array}$ & $\begin{array}{c}\text { Unpaved } \\
\text { Mileage in } \\
2000\end{array}$ & $\begin{array}{c}\text { Estimated } \\
\text { Needs in } \\
1998 \\
\text { ( Year 2002) }\end{array}$ & $\begin{array}{c}\text { Estimated } \\
\text { Needs in } \\
2000 \\
\text { (\$Y Year 2002) }\end{array}$ \\
\hline Adams & 333.73 & 333.73 & 339.75 & 339.75 \\
\hline Hamilton & 1.08 & 1.18 & 1.10 & 1.20 \\
\hline Fountain & 472.50 & 412.88 & 480.47 & 420.34 \\
\hline Lawrence & 10.00 & 10.00 & 10.18 & 10.18 \\
\hline Pike & 401.01 & 388.85 & 408.26 & 395.88 \\
\hline Fayette & 73.00 & 75.28 & 74.32 & 76.63 \\
\hline Floyd & 2.05 & 2.05 & 2.08 & 2.08 \\
\hline White & 364.17 & 364.17 & 370.75 & 370.75 \\
\hline
\end{tabular}

This methodology based on a fixed unit cost per mile of unpaved road was used due to the fact that specific data concerning unpaved roads in Indiana is not available. The study by Riverson (1983) was the only comprehensive study on the preservation and maintenance costs of unpaved roads in Indiana that could be directly used without having an expensive and time consuming data collection.

\section{Paved Roads}

Time strategies were used to determine the future pavement needs for the study period. Surface treatments and overlays were considered at different time intervals and the annual cost of preservation was estimated using average unit costs per mile.

The total needs for the eight-county sample were determined for the years 1998 and 2000 due to data limitations. Two different maintenance strategies were considered:

o Strategy 1: Surface treatment every 4.5 years and Overlays every 12 years

o Strategy 2: Surface treatment every 5 years and Overlays every 14 years

The total annual preservation cost was determined for the eight counties for 1998 and 2000 for each maintenance cycle and added to routine and preventive maintenance costs. These costs were estimated to be close to the net maintenance and repair expenses for each county available in the annual operational reports. The cost used for these calculations are $\$ 6,061$ per mile and $\$ 42,250$ per mile respectively for maintenance and reconstruction. They are based on the costs experienced in the eight counties. The obtained values are shown on Tables 2.48 and 2.49. 
Table 2.48: Estimated Needs for Preservation of Paved Roads in Sample Counties in 1998 and 2000 (\$1000)

Maintenance Strategy 1 (\$ Year 2002)

\begin{tabular}{|l|c|c|c|c|}
\hline \multirow{2}{*}{ County } & \multicolumn{2}{|c|}{1998} & \multicolumn{2}{|c|}{2000} \\
\cline { 2 - 5 } & $\begin{array}{c}\text { Paved } \\
\text { Mileage } \\
\text { (miles) }\end{array}$ & $\begin{array}{c}\text { Estimated } \\
\text { Needs }\end{array}$ & $\begin{array}{c}\text { Paved } \\
\text { Mileage } \\
\text { (miles) }\end{array}$ & $\begin{array}{c}\text { Estimated } \\
\text { Needs }\end{array}$ \\
\hline Adams & 354.47 & $\$ 3,290$ & 354.47 & $\$ 3,780$ \\
\hline Hamilton & 924.67 & $\$ 12,427$ & 894.85 & $\$ 7,759$ \\
\hline Fountain & 207.02 & $\$ 2,253$ & 264.00 & $\$ 2,484$ \\
\hline Lawrence & 657.00 & $\$ 4,509$ & 657.00 & $\$ 5,842$ \\
\hline Pike & 141.35 & $\$ 1,091$ & 153.78 & $\$ 1,586$ \\
\hline Fayette & 313.50 & $\$ 3,298$ & 305.72 & $\$ 1,887$ \\
\hline Floyd & 314.58 & $\$ 2,108$ & 318.70 & $\$ 2,214$ \\
\hline White & 580.33 & $\$ 3,743$ & 580.33 & $\$ 5,123$ \\
\hline
\end{tabular}

Table 2.49 : Estimated Needs for Preservation of Paved Roads in Sample Counties in 1998 and $2000(\$ 1000)$

Maintenance Strategy 2 (\$ Year 2002)

\begin{tabular}{|l|c|c|c|c|}
\hline \multirow{2}{*}{ County } & \multicolumn{2}{|c|}{1998} & \multicolumn{2}{|c|}{2000} \\
\cline { 2 - 5 } & $\begin{array}{c}\text { Paved } \\
\text { Mileage } \\
\text { (miles) }\end{array}$ & $\begin{array}{c}\text { Estimated } \\
\text { Needs }\end{array}$ & $\begin{array}{c}\text { Paved } \\
\text { Mileage } \\
\text { (miles) }\end{array}$ & $\begin{array}{c}\text { Estimated } \\
\text { Needs }\end{array}$ \\
\hline Adams & 354.47 & $\$ 3,085.99$ & 354.47 & $\$ 3,576.62$ \\
\hline Hamilton & 924.67 & $\$ 11,896.00$ & 894.85 & $\$ 7,228.41$ \\
\hline Fountain & 207.02 & $\$ 2,134.19$ & 264.00 & $\$ 2,332.27$ \\
\hline Lawrence & 657.00 & $\$ 4,130.23$ & 657.00 & $\$ 5,463.97$ \\
\hline Pike & 141.35 & $\$ 1,009.70$ & 153.78 & $\$ 1,496.27$ \\
\hline Fayette & 313.50 & $\$ 3,116.46$ & 305.72 & $\$ 1,710.60$ \\
\hline Floyd & 314.58 & $\$ 1,926.97$ & 318.70 & $\$ 2,029.56$ \\
\hline White & 580.33 & $\$ 3,408.00$ & 580.33 & $\$ 4,788.47$ \\
\hline
\end{tabular}




\section{Disbursements for Sample Counties}

In order to find a ratio between the estimated needs and the level of disbursements, the data on net maintenance, repair, construction and reconstruction was considered for 8 sample counties for the years 1998 and 2000. The data used was obtained from the county operational reports, submitted every year to the state authorities. The observed values are shown in Tables 2.50 and 2.51 . 
Table 2.50: Net Maintenance and Repair/Construction and Reconstruction Disbursements in Sample Counties in 1998 (\$1000 in \$ Year 2002)

\begin{tabular}{|c|c|c|c|c|c|c|c|c|c|}
\hline \multirow{2}{*}{ County } & \multicolumn{4}{|c|}{ Net Maintenance and Repair Expenses } & \multicolumn{4}{|c|}{ Construction and Reconstruction } & \multirow{2}{*}{$\begin{array}{c}\text { Total } \\
\text { Disbursements }\end{array}$} \\
\hline & MVHA & LRSF & $\begin{array}{c}\text { Other } \\
\text { Funds } \\
\text { (highway } \\
\text { dedicated) }\end{array}$ & $\begin{array}{c}\text { Total Net } \\
\text { Maintenanc } \\
\text { e and } \\
\text { Repair } \\
\text { Expenses } \\
\end{array}$ & MVHA & LRSF & $\begin{array}{l}\text { Other } \\
\text { Funds } \\
\text { (highway } \\
\text { dedicated) }\end{array}$ & $\begin{array}{l}\text { Total Net } \\
\text { Maintenance } \\
\text { and Repair } \\
\text { Expenses }\end{array}$ & \\
\hline Adams & $\$ 1,226$ & $\$ 477$ & - & $\$ 1,704$ & $\$ 77$ & $\$ 330$ & $\$ 588$ & $\$ 995$ & $\$ 2,699$ \\
\hline Hamilton & $\$ 1,318$ & $\$ 1,339$ & $\$ 5,670$ & $\$ 8,328$ & - & $\$ 869$ & $\$ 13,396$ & $\$ 14,265$ & $\$ 22,593$ \\
\hline Fountain & $\$ 981$ & $\$ 343$ & - & $\$ 1,324$ & $\begin{array}{l}\text { Included in } \\
\text { maintenance }\end{array}$ & $\begin{array}{l}\text { Included in } \\
\text { maintenance }\end{array}$ & $\begin{array}{l}\text { Included in } \\
\text { maintenance }\end{array}$ & - & $\$ 1,324$ \\
\hline Lawrence & $\$ 900$ & $\$ 673$ & - & $\$ 1,574$ & $\$ 885$ & $\$ 673$ & - & $\$ 1,559$ & $\$ 3,133$ \\
\hline Pike & $\$ 367$ & $\$ 93$ & - & $\$ 461$ & $\$ 96$ & $\$ 188$ & $\$ 115$ & $\$ 401$ & $\$ 862$ \\
\hline Fayette & $\$ 1,067$ & $\$ 821$ & - & $\$ 1,888$ & - & $\$ 579$ & - & $\$ 579$ & $\$ 2,468$ \\
\hline Floyd & $\$ 702$ & - & - & $\$ 702$ & - & $\$ 954$ & - & $\$ 954$ & $\$ 1,656$ \\
\hline White & $\$ 976$ & $\$ 177$ & - & $\$ 1,153$ & $\$ 138$ & $\$ 187$ & - & $\$ 325$ & $\$ 1,479$ \\
\hline
\end{tabular}

Source: County Operational Reports (1998) 
Table 2.51: Net Maintenance and Repair/Construction and Reconstruction Disbursements in Sample Counties in 2000 (\$1000 in \$ Year 2002)

\begin{tabular}{|c|c|c|c|c|c|c|c|c|c|}
\hline \multirow{2}{*}{ County } & \multicolumn{4}{|c|}{ Net Maintenance and Repair expenses } & \multicolumn{4}{|c|}{ Construction and Reconstruction } & \multirow{2}{*}{$\begin{array}{c}\text { Total } \\
\text { Disbursements }\end{array}$} \\
\hline & MVHA & LRSF & $\begin{array}{c}\text { Other } \\
\text { Funds } \\
\text { (highway } \\
\text { dedicated) }\end{array}$ & $\begin{array}{c}\text { Total net } \\
\text { Maintenance } \\
\text { and Repair } \\
\text { Expenses } \\
\end{array}$ & MVHA & LRSF & $\begin{array}{c}\text { Other Funds } \\
\text { (highway } \\
\text { dedicated) }\end{array}$ & $\begin{array}{c}\text { Total net } \\
\text { Maintenance } \\
\text { and Repair } \\
\text { Expenses } \\
\end{array}$ & \\
\hline Adams & $\$ 1,331$ & $\$ 860$ & - & $\$ 2,192$ & $\$ 318$ & $\$ 621$ & - & $\$ 940$ & $\$ 3,132$ \\
\hline Hamilton & $\$ 1,432$ & $\$ 2,228$ & - & $\$ 3,661$ & - & $\$ 549$ & $\$ 4,387$ & $\$ 4,936$ & $\$ 8,598$ \\
\hline Fountain & $\$ 921$ & $\$ 380$ & - & $\$ 1,301$ & $\begin{array}{l}\text { Included in } \\
\text { maintenance }\end{array}$ & $\begin{array}{l}\text { Included in } \\
\text { maintenance }\end{array}$ & $\begin{array}{l}\text { Included in } \\
\text { maintenance }\end{array}$ & - & $\$ 1,301$ \\
\hline Lawrence & $\$ 1,849$ & $\$ 1,040$ & $\$ 9$ & $\$ 2,899$ & - & $\$ 693$ & - & $\$ 693$ & $\$ 3,593$ \\
\hline Pike & $\$ 417$ & $\$ 183$ & $\$ 294$ & $\$ 895$ & $\$ 101$ & $\$ 164$ & - & $\$ 265$ & $\$ 1,161$ \\
\hline Fayette & $\$ 301$ & $\$ 219$ & - & $\$ 521$ & - & $\$ 714$ & $\$ 454$ & $\$ 1,169$ & $\$ 1,690$ \\
\hline Floyd & $\$ 770$ & - & $\$ 18$ & $\$ 788$ & - & $\$ 636$ & - & $\$ 636$ & $\$ 1,425$ \\
\hline White & $\$ 2,297$ & $\$ 228$ & - & $\$ 2,525$ & $\$ 122$ & $\$ 404$ & - & $\$ 526$ & $\$ 3,052$ \\
\hline
\end{tabular}

Source: County Operational Reports (2000) 
From Tables 2.50 and 2.51 it can be observed that the Hamilton County had very high level of supplemental funding from other sources as compared to the other counties; as it can be seen that it had an approximate spending of $\$ 13$ million in 1998 and $\$ 4.3$ million in 2000 on maintenance, coming from supplemental funds. In the sample of eight counties, for the same years, the total supplemental funding for the other seven counties totaled $\$ 692,000$ in 1998 and $\$ 447,000$ in 2000. In general, for most of the counties, the supplemental funding is small and does not provide a sufficient contribution to road maintenance. More recently, in 2001, the supplemental revenue Hamilton County received attained $\$ 16$ million and constituted approximately $13 \%$ of the total supplemental revenue for all 92 counties. In order to obtain a better representation of the state, Hamilton County was therefore excluded from the comparative analysis of disbursements and needs.

\section{Determination of Needs / Disbursements Ratio}

The total needs for both paved and unpaved roads were added and compared to the total disbursements (net maintenance and repair expenses and construction and reconstruction) for the sample of seven counties, excluding Hamilton County. The gap was also calculated and a ratio between needs and disbursements was determined for the two different maintenance strategies. The values found under Maintenance Strategy 1 are shown on Tables 2.52 and 2.53. Corresponding values under Maintenance Strategy 2 are given in Tables 2.54 and 2.55.

Table 2.52: Difference between Disbursements and Needs in 1998 in $\$ 1000$ Maintenance Strategy 1 (\$ Year 2002)

\begin{tabular}{|l|c|c|c|}
\hline & Disbursements & Needs & Gap \\
\hline Adams & $\$ 2,699$ & $\$ 3,290$ & $\$ 591$ \\
\hline Fountain & $\$ 1,324$ & $\$ 2,253$ & $\$ 929$ \\
\hline Lawrence & $\$ 3,133$ & $\$ 4,509$ & $\$ 1,376$ \\
\hline Pike & $\$ 862$ & $\$ 1,091$ & $\$ 229$ \\
\hline Fayette & $\$ 2,468$ & $\$ 3,298$ & $\$ 830$ \\
\hline Floyd & $\$ 1,656$ & $\$ 2,108$ & $\$ 452$ \\
\hline White & $\$ 1,479$ & $\$ 3,743$ & $\$ 2,264$ \\
\hline Total & $\$ 13,621$ & $\$ 20,292$ & $\$ 6,671$ \\
\hline
\end{tabular}


Table 2.53: Difference between Disbursements and Needs in 2000 in $\$ 1000$ Maintenance Strategy 1 (\$ Year 2002)

\begin{tabular}{|l|c|c|c|}
\hline & Disbursements & Needs & Gap \\
\hline Adams & $\$ 3,132$ & $\$ 3,780$ & $\$ 648$ \\
\hline Fountain & $\$ 1,301$ & $\$ 2,484$ & $\$ 1,183$ \\
\hline Lawrence & $\$ 3,593$ & $\$ 5,842$ & $\$ 2,249$ \\
\hline Pike & $\$ 1,161$ & $\$ 1,586$ & $\$ 425$ \\
\hline Fayette & $\$ 1,690$ & $\$ 1,887$ & $\$ 197$ \\
\hline Floyd & $\$ 1,425$ & $\$ 2,214$ & $\$ 789$ \\
\hline White & $\$ 3,052$ & $\$ 5,123$ & $\$ 2,071$ \\
\hline Total & $\$ 15,354$ & $\$ 22,916$ & $\$ 7,562$ \\
\hline
\end{tabular}

Table 2.54: Difference between Disbursements and Needs in 1998 in $\$ 1000$ Maintenance Strategy 2 (\$ Year 2002)

\begin{tabular}{|l|c|c|c|}
\hline & Disbursements & Needs & Gap \\
\hline Adams & $\$ 2,699$ & $\$ 3,085.99$ & $\$ 386.99$ \\
\hline Fountain & $\$ 1,324$ & $\$ 2,134.19$ & $\$ 810.19$ \\
\hline Lawrence & $\$ 3,133$ & $\$ 4,130.23$ & $\$ 997.23$ \\
\hline Pike & $\$ 862$ & $\$ 1,009.70$ & $\$ 147.70$ \\
\hline Fayette & $\$ 2,468$ & $\$ 3,116.46$ & $\$ 648.46$ \\
\hline Floyd & $\$ 1,656$ & $\$ 1,926.97$ & $\$ 270.97$ \\
\hline White & $\$ 1,479$ & $\$ 3,408.00$ & $\$ 1,929.00$ \\
\hline Total & $\$ 13,621$ & $\$ 18,811.54$ & $\$ 5,190.54$ \\
\hline
\end{tabular}


Table 2.55: Difference between Disbursements and Needs in 2000 in $\$ 1000$ Maintenance Strategy 2 (\$Year 2002)

\begin{tabular}{|l|c|c|c|}
\hline & Disbursements & Needs & Gap \\
\hline Adams & $\$ 3,132$ & $\$ 3,576.62$ & $\$ 444.62$ \\
\hline Fountain & $\$ 1,301$ & $\$ 2,332.27$ & $\$ 1,031.27$ \\
\hline Lawrence & $\$ 3,593$ & $\$ 5,463.97$ & $\$ 1,870.97$ \\
\hline Pike & $\$ 1,161$ & $\$ 1,496.27$ & $\$ 335.27$ \\
\hline Fayette & $\$ 1,690$ & $\$ 1,710.60$ & $\$ 20.60$ \\
\hline Floyd & $\$ 1,425$ & $\$ 2,029.56$ & $\$ 604.56$ \\
\hline White & $\$ 3,052$ & $\$ 4,788.47$ & $\$ 1,736.47$ \\
\hline Total & $\$ 15,354$ & $\$ 21,398$ & $\$ 6,044$ \\
\hline
\end{tabular}

For each maintenance strategy alternative, an average ratio between the years 1998 and 2000 was determined, as shown below.

Average Ratio of Needs and Disbursements $=$

((Needs/Disbursements) ${ }_{1998}+$ (Needs/Disbursements) 2000)/2

- $\quad$ Maintenance Strategy 1: Ratio $=1.49$

- $\quad$ Maintenance Strategy 2: Ratio $=1.38$

Using these ratios, a range of values for the physical needs over the study period for the entire state could be derived. The projected disbursements were then used to estimate needs, as shown below:

Needs $=$ Ratio $*$ Disbursements

Disbursement projection procedures and results are given in Appendix $\mathrm{H}$.

\section{Estimation of Needs for All 92 Counties}

Using data from Highway Statistics publications (BTS 1995-2001), disbursements in Indiana counties could be projected on the basis of VMT over the study period. As specified above, the average ratios were used and a range of values for physical needs could be determined for each year. Table 2.56 presents the estimated needs over the study period (2006-2020). 
Table 2.56: Estimated Fiscal Needs for Local Roads

(\$Year 2002)

\begin{tabular}{|c|c|c|}
\hline Year & $\begin{array}{c}\text { Projected } \\
\text { Disbursements } \\
\text { (\$ Million) }\end{array}$ & $\begin{array}{c}\text { Range of Estimated } \\
\text { Needs } \\
\text { (\$ Million) }\end{array}$ \\
\hline 2006 & 823 & $1,136-1,226$ \\
\hline 2007 & 855 & $1,180-1,274$ \\
\hline 2008 & 887 & $1,224-1,321$ \\
\hline 2009 & 919 & $1,268-1,369$ \\
\hline 2010 & 951 & $1,312-1,417$ \\
\hline 2011 & 983 & $1,356-1,464$ \\
\hline 2012 & 1,015 & $1,400-1,512$ \\
\hline 2013 & 1,047 & $1,445-1,560$ \\
\hline 2014 & 1,079 & $1,489-1,607$ \\
\hline 2015 & 1,111 & $1,533-1,655$ \\
\hline 2016 & 1,143 & $1,577-1,703$ \\
\hline 2017 & 1,175 & $1,621-1,750$ \\
\hline 2018 & 1,207 & $1,665-1,798$ \\
\hline 2019 & 1,239 & $1,710-1,846$ \\
\hline 2020 & 1,272 & $1,755-1,895$ \\
\hline \multicolumn{3}{|c|}{ TOTAL $=\$ 21,671-23,402$ Million } \\
\hline
\end{tabular}

A total of $\$ 21.7$ to $\$ 23.4$ billion is estimated as the needs for the preservation and maintenance of local roads and streets for 2006-2020, on the basis of the Need/Disbursement ratios of 1.38 and 1.49 estimated for a sample of seven counties, respectively. Figure 2.15 illustrates the obtained values of needs and disbursements for the study period. The principal disadvantage of this approach for needs estimation is that over the years the monetary needs estimated are increasing. In actual situation the system should be upgraded to an acceptable condition, which is defined as "backlog", and then preservation actions should be performed on yearly basis. However, the method provides a good estimation of aggregated future needs for the entire study period. 


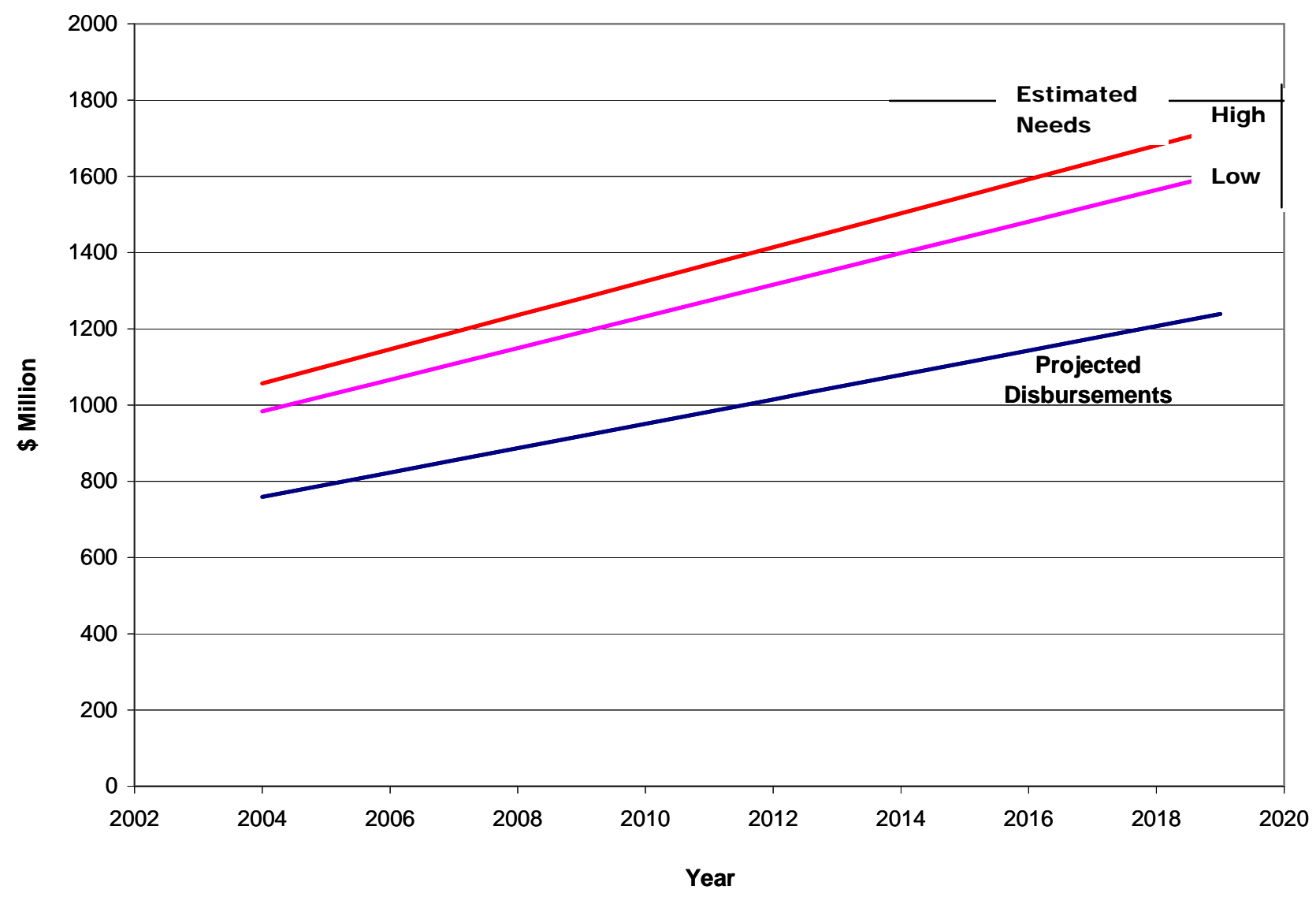

Figure 2.15: Estimated Fiscal Needs and Projected Disbursements (2002 dollars)

\subsubsection{Estimation of "Backlog" Using the Trigger Value Approach (Paved Road)}

The approach used here to determine immediate needs is based on pavement condition. Two different sources of data were used for rural and urban roads, respectively. The survey carried out by LTAP in 2001 included 3,200 miles of county roads which provided data on pavement condition for rural roads. For urban roads, the data was provided by INDOT from the HPMS (Highway Performance Monitoring System) file representing both the state and local highway system. The specific data representing local urban road condition in Indiana has been isolated. The data used included 127 lane miles of roads. For both rural and urban roads, IRI (International Roughness Index) values were considered as a measure of pavement condition.

The IRI measures the cumulative deviation from a smooth surface in inches per mile. It is commonly used to evaluate pavement performance based upon considerations of riding quality. The higher the IRI value, the rougher is the ride. A review of sample data in INDIPAVE (2000) suggests 
a new flexible pavement would have an initial IRI of 60 and typical new rigid pavement would have an initial IRI of 70. Among developed measures of pavement performance, IRI has enjoyed the wide application and has been adopted as a standard for the Federal Highway Performance Monitoring System (FHWA, 1987). A summary of the IRI index as provided by the Pavement Management Section of the Program Development Division of INDOT is shown in Table 2.57 below. In the present study, IRI values were available for both the data sets used for the urban and rural systems.

Table 2.57: Pavement Condition and IRI Values

\begin{tabular}{cc}
\hline Pavement Condition & IRI Range \\
\hline Excellent & $60-100$ \\
Good & $100-150$ \\
Fair & $150-200$ \\
Poor & $>200$ \\
\hline
\end{tabular}

Source: INDOT, 2000

INDOT defines pavements in "good" condition as those with IRI values less than 100 inches/mile. However, for the local system, due to low volume traffic, the standards can be relaxed. The roughness value of 150 inches/mile was considered to be the desired condition. To assess the backlog, all pavements with IRI values greater than 150 inches/mile were considered as needing immediate maintenance action. Pavements with IRI values greater than 200 inches/miles were eligible for reconstruction and pavements in the range 150-200 inches/mile need rehabilitation actions. Depending on whether the threshold value is fixed to 150 inches/mile or 200 inches/mile, a range of values was estimated as the backlog for both urban and rural roads. Table 2.58 illustrates the IRI threshold values corresponding to the level of preservation chosen for local pavements.

Table 2.58: IRI Threshold Value Corresponding to Level of Maintenance for Local Pavements

\begin{tabular}{|c|c|}
\hline Level of Maintenance Chosen & IRI Threshold Value (inches/mile) \\
\hline $\begin{array}{c}\text { Best case scenario: Upgrade all local } \\
\text { pavements in "poor" and "fair" condition to a } \\
\text { "good" condition }\end{array}$ & 150 \\
\hline $\begin{array}{c}\text { Worse case scenario: Repair all pavements in } \\
\text { "poor" condition }\end{array}$ & 200 \\
\hline
\end{tabular}

\section{Rural Road Needs}

The results of the backlog assessment for local rural roads are presented in Table 2.59. The unit costs per mile used for reconstruction and maintenance were taken from the LTAP study (LTAP 2001). The condition survey performed by LTAP identified $50 \%$ of local rural roads as 
having IRI values higher than 200 (poor condition), and $36 \%$ of local rural roads as being in a fair condition (IRI 150 to 200).

Table 2.59: Backlog Pavement Preservation Needs for Rural Roads (2001)

\begin{tabular}{|c|c|c|c|c|c|c|}
\hline $\begin{array}{l}\text { Condition } \\
\text { IRI }\end{array}$ & $\begin{array}{c}\text { Desired } \\
\text { Condition } \\
(\mathrm{IRI}=125)\end{array}$ & Percentage & Mileage & Treatment & $\begin{array}{l}\text { Unit Cost } \\
\text { (\$/mile) }\end{array}$ & Total Cost \\
\hline $\begin{array}{c}\text { Excellent } \\
\text { (IRI }=60- \\
100)\end{array}$ & 150 & $6 \%$ & 2,290 & Do Nothing & $\mathrm{n} / \mathrm{a}$ & $\mathrm{n} / \mathrm{a}$ \\
\hline $\begin{array}{c}\text { Good } \\
(\mathrm{IRI}=101- \\
150)\end{array}$ & 150 & $16 \%$ & 5,954 & Do Nothing & $\mathrm{n} / \mathrm{a}$ & $\mathrm{n} / \mathrm{a}$ \\
\hline $\begin{array}{c}\text { Fair } \\
(\mathrm{IRI}=151- \\
200) \\
\end{array}$ & 150 & $29.5 \%$ & 11,282 & $\begin{array}{l}\text { Preventive } \\
\text { Maintenance }\end{array}$ & 6,191 & $\begin{array}{l}\$ 70 \\
\text { Million }\end{array}$ \\
\hline $\begin{array}{c}\text { Poor } \\
(I R I=>200)\end{array}$ & 150 & $48.4 \% \%$ & 18,492 & Reconstruction & 43,154 & $\begin{array}{l}\$ 798 \\
\text { million }\end{array}$ \\
\hline $\begin{array}{c}\text { All } \\
\text { Conditions }\end{array}$ & $\mathrm{n} / \mathrm{a}$ & $\mathrm{n} / \mathrm{a}$ & 38,167 & $\begin{array}{c}\text { Routine } \\
\text { Maintenance }\end{array}$ & $\$ 85$ & $\begin{array}{l}\$ 3.2 \\
\text { million }\end{array}$ \\
\hline \multicolumn{7}{|c|}{ Total preservation cost for rural roads (backlog) $=\$ 871$ million } \\
\hline
\end{tabular}

Source: Road Condition Survey (LTAP 2001). All costs are in 2002\$

\section{Urban Road Needs}

For urban pavements, the same methodology was applied and the same threshold values were used, but the data used was provided by the FHWA Highway Pavement Monitoring System (HPMS) database. A sample representative of local urban roads in the entire state was used. Urban roads often have more than one lane in each direction; therefore costs per mile can not be used. From a sample of 11 values, an average cost of $\$ 85$ per square yard can be found for reconstruction. A unit cost of $\$ 12$ per square yard was then derived for preventive maintenance, using the ratio existing between reconstruction costs and preventive maintenance costs for rural roads. Assuming that the average width of a local urban pavement is 12 feet including shoulders, the unit cost per square yard was converted to unit cost per lane mile. A cost of $\$ 84,440$ per lane mile was estimated for maintenance and a cost of $\$ 598,400$ per lane mile was determined for reconstruction. Using these unit costs, all operations to upgrade pavements conditions were estimated for the sample considered, and then a preservation unit cost per lane mile was derived for urban roads. The results are shown on Table 2.60. A preservation unit cost of $\$ 12,392$ per lane mile was found for urban roads on the local system. Assuming that typically an urban local road has 2 lanes, the total number of lane miles for the 
urban local system was estimated to be 27,833 and hence a total preservation cost obtained was $\$ 345$ million.

Table 2.60: Backlog Pavement Preservation Needs for Urban Roads in Sample Data (\$ Year 2002)

\begin{tabular}{|c|c|c|c|c|c|c|}
\hline $\begin{array}{l}\text { Condition } \\
\text { IRI }\end{array}$ & $\begin{array}{c}\text { Desired } \\
\text { Condition } \\
(\mathrm{IRI}=125) \\
\end{array}$ & $\begin{array}{c}\text { Existing } \\
\text { Average } \\
\text { IRI }\end{array}$ & $\begin{array}{l}\text { Number of } \\
\text { Lane miles }\end{array}$ & Treatment & $\begin{array}{l}\text { Unit Cost } \\
\text { (\$/ lane mile) }\end{array}$ & Total cost \\
\hline $\begin{array}{c}\text { Excellent } \\
\text { (IRI= 60- } \\
100)\end{array}$ & 150 & 87 & 35 & Do Nothing & $\mathrm{n} / \mathrm{a}$ & $\mathrm{n} / \mathrm{a}$ \\
\hline $\begin{array}{c}\text { Good } \\
(\mathrm{IRI}=101- \\
150)\end{array}$ & 150 & 127 & 81 & Do Nothing & $\mathrm{n} / \mathrm{a}$ & $\mathrm{n} / \mathrm{a}$ \\
\hline $\begin{array}{c}\text { Fair } \\
\text { (IRI=151- } \\
200)\end{array}$ & 150 & 177 & 10 & $\begin{array}{l}\text { Preventative } \\
\text { Maintenance }\end{array}$ & $\$ 84,440$ & $\begin{array}{l}\$ 0.844 \\
\text { million }\end{array}$ \\
\hline $\begin{array}{c}\text { Poor } \\
(\text { IRI }=>200)\end{array}$ & 150 & 291 & 1.2 & Reconstruction & $\$ 598,400$ & $\begin{array}{l}\$ 0.718 \\
\text { million }\end{array}$ \\
\hline $\begin{array}{c}\text { All } \\
\text { Conditions }\end{array}$ & $\mathrm{n} / \mathrm{a}$ & $\mathrm{n} / \mathrm{a}$ & 127 & $\begin{array}{c}\text { Routine } \\
\text { Maintenance }\end{array}$ & $\$ 85$ & $\$ 10,830$ \\
\hline
\end{tabular}

Source: Indiana Department of Transportation (INDOT), Highway Performance Monitoring System (HPMS) data base (2002)

\subsubsection{Estimation of "Backlog" Using the Annual Average Maintenance Expenditure (AAMEX) Approach (Unpaved Road)}

The method used to assess the unpaved road needs for the sample of eight counties was also used to determine the backlog for local unpaved roads statewide. The average unit costs derived from an earlier study (Riverson 1983) were used. Unpaved roads account for $35.5 \%$ of the local road mileage, representing 28,874 miles. Using the average unit cost for preservation of unpaved roads (Section 2.2.1.1), the total preservation needs for local unpaved roads can be estimated as $\$ 29$ million per year.

\subsubsection{Summary of Backlog Assessment for Local Roads and Pavement}

Table 2.61 summarizes the current preservation needs for roads on the local system. The immediate short term need defined as "backlog." 
Table 2.61: Backlog Preservation Needs for Local Roads

\begin{tabular}{|l|c|c|}
\hline & Urban & Rural \\
\hline Paved Road Mileage & 27,833 lane-miles & 38,167 miles \\
\hline Pavement Preservation Needs & $\begin{array}{c}\$ 160 \text { to } \$ 345 \\
\text { million }\end{array}$ & $\begin{array}{c}\$ 801 \text { to } \$ 871 \\
\text { Million }\end{array}$ \\
\hline Unpaved Road Mileage & 686 miles & 28,188 miles \\
\hline $\begin{array}{l}\text { Unpaved Road Preservation } \\
\text { Needs }\end{array}$ & 0.7 Million & 28.7 Million \\
\hline \multicolumn{2}{|c|}{ TOTAL COST (backlog) $=\$ \mathbf{0 . 9 6}$ to $\$ \mathbf{1 . 2}$ billion } \\
\hline
\end{tabular}

\subsubsection{Summary of Pavements Needs Assessment}

In the present study, a ratio between needs and disbursements was first determined, and then the total local road pavement needs were estimated over the study period for all Indiana counties. Furthermore, the backlog which constitutes the immediate needs required to upgrade the system to an acceptable level was also estimated on the basis of existing pavement condition. Table 2.62 summarizes the total needs and the backlog estimated in the study.

Table 2.62: Summary of Pavement Needs for Indiana Local Roads (2006-2020)

\begin{tabular}{|c|c|}
\hline Backlog (as of 2006) & \$0.96 to \$1.2 Billion \\
\hline $\begin{array}{c}\text { Total Needs including Backlog } \\
\text { over the Study Period (2006-020) }\end{array}$ & $\$ 21.6$ to \$23.4 Billion \\
\hline
\end{tabular}

All costs are expressed in $2002 \$$

\subsubsection{Need Assessment for Local Bridges}

\section{Existing Conditions}

Local highway agencies in Indiana maintain 12,549 bridges which are more than 20 feet in length and over 180,000 smaller bridges and culverts. Among the total of 12,549 local bridges, $43 \%$ are considered deficient, either Structurally Deficient (SD) or Functionally Obsolete (FO). A 
structurally deficient bridge is restricted to light vehicles due to deterioration of the bridge components. A functionally obsolete bridge is a bridge where deck geometry, load capacity or roadway alignment is less than present desirable criteria for the roadway on which it is located. A bridge could be in good condition, even newly constructed, but still be considered functionally obsolete due to poor design or planning.

The Sufficiency Rating (SR) is one of the primary factors considered in determining whether federal funds may be used to replace a given structure. The SR of a bridge is a numerical rating, on a scale of 0 (poor) to 100 (excellent) which is a measure of the sufficiency of the structure to remain in service. It is calculated based on data collected from the National Bridge Inventory and includes factors such as condition, bridge geometry, traffic volumes, and the length of alternate routes. Federal Highway Administration rules require that a bridge must have a SR of less than 50 to be eligible for replacement, and less than 80 to be eligible for rehabilitation.

In Indiana, on average $13.8 \%$ of local bridges have SR less than 50 which represent approximately 1,738 bridges, and $21.4 \%$ of bridges have SR between 50 and 80, representing 2,683 bridges. Table 2.63 presents a summary of average sufficiency ratings for local bridges, as of 20002001 (Appendix I presents bridge sufficiency ratings by county).

Table 2.63: Summary Local Bridge Sufficiency Ratings (2000-2001)

\begin{tabular}{|l|c|c|}
\hline & Number & Percentage \\
\hline Low SR 0-49.9 & 1,738 & $13.8 \%$ \\
\hline Medium SR 50-79.9 & 2,683 & $21.4 \%$ \\
\hline High SR 80-100 & 8,106 & $64.7 \%$ \\
\hline Total & 12,527 & $100 \%$ \\
\hline \multicolumn{2}{|c|}{ Average SR $=78.85$} \\
\hline \multicolumn{2}{|c|}{ Total Deck Area $=27,021,130$} \\
\hline
\end{tabular}

\subsubsection{Need Assessment on the Basis of Sufficiency Ratings (Assessment of Backlog)}

\section{Replacement Needs}

To assess the bridge replacement needs, an Indiana based study (Elridge 2002) was used, where a model for bridge replacement cost was developed as a function of three variables: ADT, deck area and funding type (Federal and non-Federal). The average cost found for local bridge was 
\$106 per square foot in 2002 dollars. Table 2.64 shows local bridge replacement costs based on the sufficiency ratings (replacement needs broken down by county can be found in Appendix J).

Table 2.64: Cost Estimations for Bridge Replacement (2000-2001)

\begin{tabular}{|l|l|}
\hline Total Deck Area (sq. ft.) & $27,021,130$ \\
\hline Area Bridges with SR 0-49.9 (sq. ft.) & $2,499,201$ \\
\hline Percentage of Bridge Deck Area with SR 0-49.9 & $9.25 \%$ \\
\hline Unit Cost Bridge Replacement & $\$ 113$ \\
\hline Total Cost of Bridge Replacement & $\mathbf{\$ 2 8 2 . 4}$ Million \\
\hline
\end{tabular}

2,499,201 square feet of bridge deck area fall in the SR range 0-49.9, making them eligible for replacement. With the unit cost of $\$ 113$ per square foot of deck area, the total local bridge replacement need for Indiana is $\$ 282.4$ million in 2002 constant dollars. The value found represents the current needs for bridge replacement.

\section{Rehabilitation Needs}

The total deck area for all bridges with a SR between 50-80 was estimated. As no unit cost value for local bridge rehabilitation was directly available, the data from state bridges was used to estimate the missing value. The average unit costs for state bridge replacement and rehabilitation were found by Rodriguez (2004) to be $\$ 190$ per square foot and $\$ 52$ per square foot respectively. Rehabilitation cost was approximately $28 \%$ of replacement cost. Using the unit cost determined for local bridge replacement, the unit cost for local bridge rehabilitation derived on the basis of the state percentage was $\$ 31$ per square foot. The costs estimation of county bridges rehabilitation based on sufficiency ratings are shown on Table 2.65 (rehabilitation needs broken down by county can be found in Appendix K).

Table 2.65: Costs Estimations for Bridge Rehabilitation (2000-2001)

\begin{tabular}{|l|l|}
\hline Total Deck Area (sq. ft.) & $27,021,130$ \\
\hline Area Bridges with SR 50-79.9 (sq. ft.) & $5,606,982$ \\
\hline Percentage of Bridge Deck Area with SR 50-79.9 & $20.75 \%$ \\
\hline Unit Cost Bridge Rehabilitation & $\$ 31$ \\
\hline Total Cost of Bridge Rehabilitation & $\$ \mathbf{1 7 3 . 8}$ Million \\
\hline
\end{tabular}


The total deck area of bridges with SR between 50 and 80 was estimated as 5,606,982 square feet. Using the unit cost of $\$ 31$ per square foot of deck area, the total cost for bridge rehabilitation was estimated at $\$ 173.8$ million (\$Year 2002). These values represent the current local need for bridge rehabilitation in Indiana. These needs constitute the current or immediate needs defined as backlog. As of 2006 , the backlog is estimated at $\$ 433$ million.

\subsubsection{Need Assessment on the Basis of Age (Assessment of Total Needs)}

The IBMS software could not be used to estimate local bridge needs due to the lack of proper input files for local bridges. Therefore bridge service life was chosen as a basis for the estimation of future needs and also to confirm the backlog. The approach considered was based on the determination of the number of bridges approaching the end of their service life and requiring a major rehabilitation. The mean age at which bridge replacement occurs is approximately 70 years for most steel and concrete bridges. Table 2.66 was developed based on the assumption that bridge replacement needs occur when bridges reach the age of 70 . According to the standards defined by INDOT, bridges should receive a major repair (deck rehabilitation, superstructure repair, or bridge rehabilitation) every 20 or 25 years in order to extend the design life to a service life of 70 to 80 years. For instance, for a typical concrete bridge, it is suggested to have deck rehabilitation after 20 years of construction and a superstructure replacement after 15 years of the previous rehabilitation. However, on a local level, counties do not rehabilitate their bridges every 20 years. Therefore, it was assumed that counties would rehabilitate bridges at least once during service life. Rehabilitation was thus assumed to occur 35 years after bridge construction. Table 2.66 also shows the number of bridges that would need a major rehabilitation due to the fact that 35 years have passed since their construction. Current bridge replacement and rehabilitation needs are included as "backlog." 
Table 2.66: Age-based Physical Needs Assessment (NBIS 2000-2001)

\begin{tabular}{|c|c|c|c|c|}
\hline Year & $\begin{array}{c}\text { Number of } \\
\text { Bridges } \\
\text { Approaching the } \\
\text { End of Service } \\
\text { Life }\end{array}$ & $\begin{array}{c}\text { Cumulative Number } \\
\text { of Bridges } \\
\text { Approaching the } \\
\text { End of Service Life }\end{array}$ & $\begin{array}{l}\text { Number of Bridges } \\
\text { with } 35 \text { years or } \\
\text { More Since Their } \\
\text { Construction }\end{array}$ & $\begin{array}{l}\text { Cumulative Number } \\
\text { of Bridges with } 35 \\
\text { years or More Since } \\
\text { Their Construction }\end{array}$ \\
\hline Backlog & 2390 & 2390 & 599 & 599 \\
\hline 2006 & 22 & 2412 & 152 & 751 \\
\hline 2007 & 26 & 2438 & 196 & 947 \\
\hline 2008 & 31 & 2469 & 176 & 1123 \\
\hline 2009 & 17 & 2486 & 236 & 1359 \\
\hline 2010 & 307 & 2793 & 332 & 1691 \\
\hline 2011 & 8 & 2801 & 271 & 1962 \\
\hline 2012 & 5 & 2806 & 215 & 2177 \\
\hline 2013 & 0 & 2806 & 189 & 2366 \\
\hline 2014 & 1 & 2807 & 193 & 2559 \\
\hline 2015 & 62 & 2869 & 269 & 2828 \\
\hline 2016 & 10 & 2879 & 172 & 3000 \\
\hline 2017 & 11 & 2890 & 228 & 3228 \\
\hline 2018 & 31 & 2921 & 208 & 3436 \\
\hline 2019 & 11 & 2932 & 220 & 3656 \\
\hline 2020 & 242 & 3174 & 224 & 3880 \\
\hline
\end{tabular}

\section{Replacement Needs}

The average deck area of 2,145 square feet was determined for all local bridges. Using a bridge replacement unit cost of $\$ 113$ per square foot of deck area, the local bridge replacement cost was estimated as shown in Table 2.67. It can be seen in Figure 2.16 that the maximum need for bridge replacement activity would occur in 2010 with a financial need of $\$ 70$ million. After that bridge replacement needs would be minimal until 2015. 
Table 2.67: Age-based Monetary Needs for Bridge Replacement (\$ Year 2002)

\begin{tabular}{|c|c|c|}
\hline Year & $\begin{array}{c}\text { Bridge } \\
\text { Deck } \\
\text { Area } \\
\text { (sq.ft.) }\end{array}$ & $\begin{array}{c}\text { Bridge } \\
\text { Replacement } \\
\text { Cost } \\
\text { (\$millions) }\end{array}$ \\
\hline Backlog & $5,126,550$ & 579.3 \\
\hline 2006 & 47,196 & 5.3 \\
\hline 2007 & 55,778 & 3.6 \\
\hline 2008 & 66,504 & 7.5 \\
\hline 2009 & 36,470 & 4.1 \\
\hline 2010 & 658,604 & 74 \\
\hline 2011 & 17,162 & 2.0 \\
\hline 2012 & 10,726 & 1.2 \\
\hline 2013 & 0 & 0 \\
\hline 2014 & 2,145 & 0.2 \\
\hline 2015 & 133,008 & 15.0 \\
\hline 2016 & 21,453 & 2.4 \\
\hline 2017 & 23,598 & 2.6 \\
\hline 2018 & 66,504 & 7.5 \\
\hline 2019 & 23,598 & 2.6 \\
\hline 2020 & 519,090 & 58.0 \\
\hline & TOTAL= $\$ 765.3$ million \\
\hline
\end{tabular}

Note: - Unit Replacement Cost per Bridge $=\$ 113$ per sq. ft. of deck area 


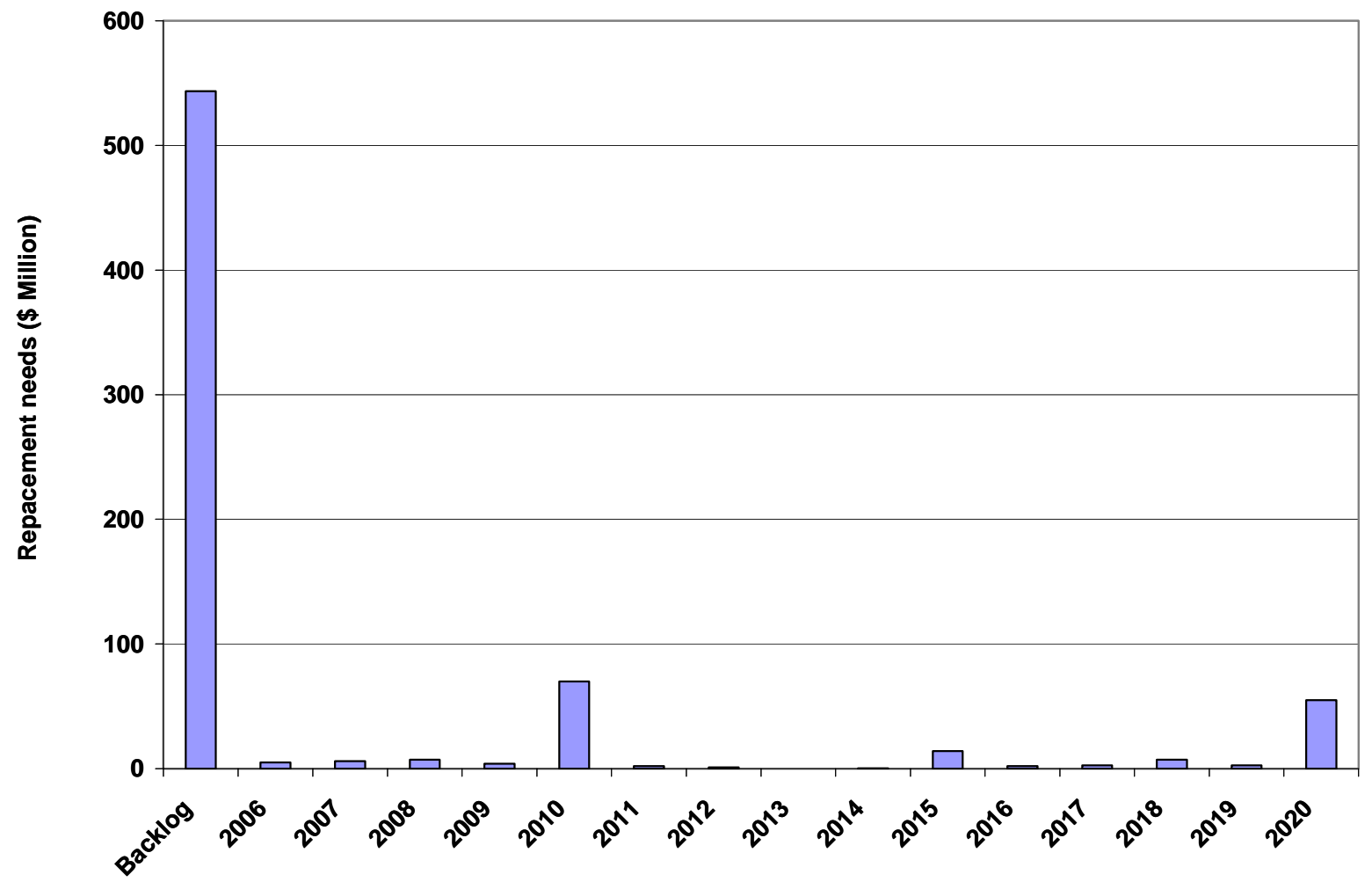

Figure 2.16: Local Bridge Replacement Monetary Needs

\section{Rehabilitation Needs}

With the average deck area of 2,145 square feet and a bridge rehabilitation unit cost of $\$ 31$ per square foot of deck area, the financial needs associated with bridge rehabilitation during 2006-2020 were estimated as shown in Table 2.68. The rehabilitation needs seem to be well distributed throughout the years after 2005 as shown in Figure 2.17. A maximum need occurs in 2010 as $\$ 22$ million, whereas the minimum need occurs in 2006 as $\$ 10$ million. 
Table 2.68: Age-based Monetary Needs for Bridge Rehabilitation (\$ Year 2002)

\begin{tabular}{|c|c|c|}
\hline Year & $\begin{array}{l}\text { Bridge Deck } \\
\text { Area (sq.ft.) }\end{array}$ & $\begin{array}{c}\text { Bridge } \\
\text { Rehabilitation } \\
\text { Cost } \\
\text { (\$millions) }\end{array}$ \\
\hline Backlog & $1,284,855$ & 389.8 \\
\hline 2006 & 326,040 & 10.0 \\
\hline 2007 & 420,420 & 13.0 \\
\hline 2008 & 377,520 & 11.7 \\
\hline 2009 & 506,220 & 15.7 \\
\hline 2010 & 712,140 & 22.0 \\
\hline 2011 & 581,295 & 18.0 \\
\hline 2012 & 461,175 & 14.2 \\
\hline 2013 & 405,405 & 12.5 \\
\hline 2014 & 413,985 & 12.8 \\
\hline 2015 & 577,005 & 17.8 \\
\hline 2016 & 368,940 & 11.4 \\
\hline 2017 & 489,060 & 15.1 \\
\hline 2018 & 446,160 & 13.8 \\
\hline 2019 & 471,900 & 14.6 \\
\hline 2020 & 480,480 & 14.8 \\
\hline \multicolumn{3}{|c|}{ Total $=\$ 257.2$ million } \\
\hline
\end{tabular}

Note: - Unit Rehabilitation Cost for Bridge $=\$ 31$ per sq. ft. of deck area 


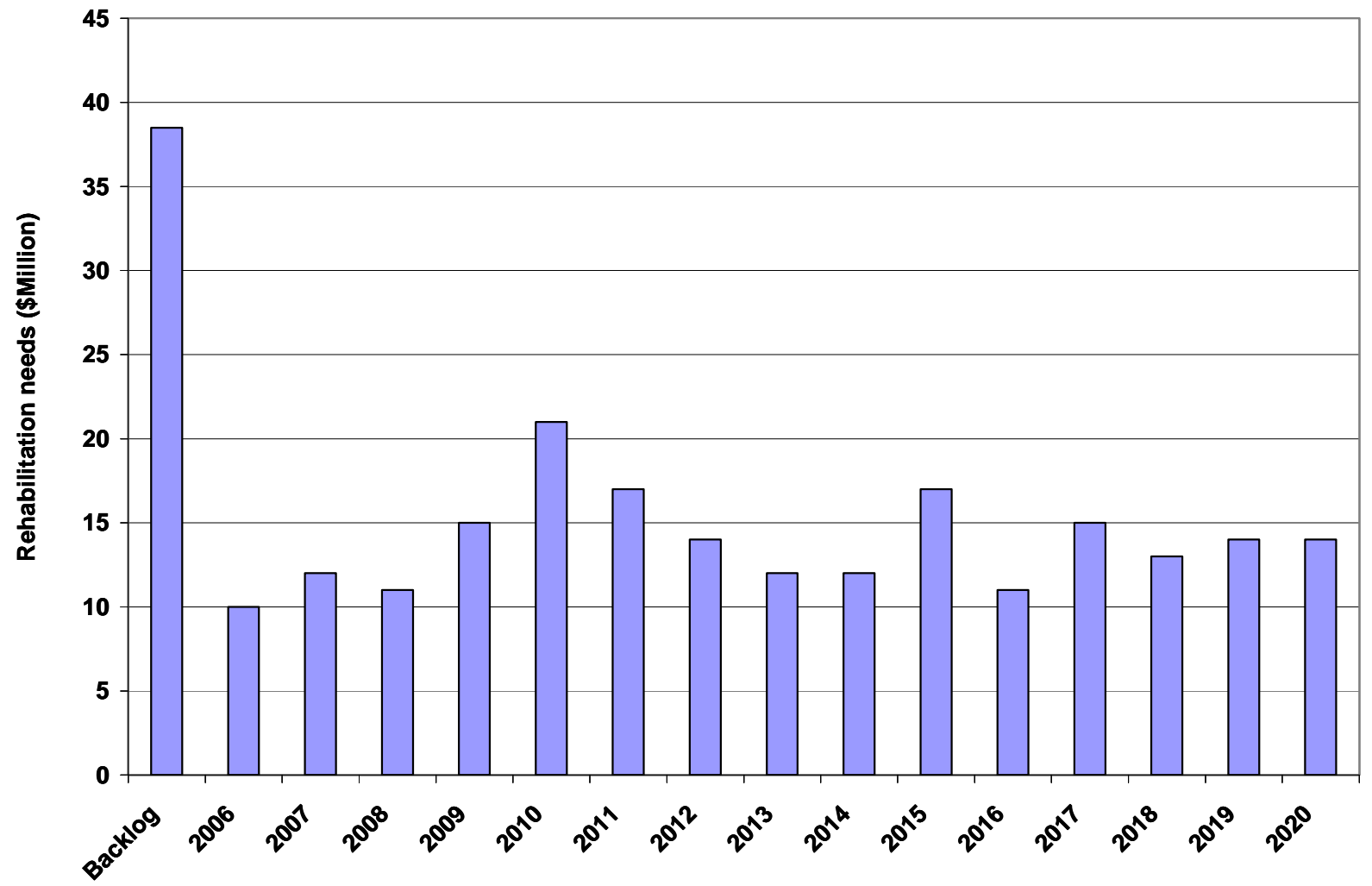

Figure 2.17: Indiana Local Bridge Rehabilitation Fiscal Needs

\subsubsection{Summary of Need Assessment for Local Bridges}

In the present study, local bridge needs were determined using two approaches. The first method was based on bridge physical condition measured by the Sufficiency Rating which allows an estimation of the current or immediate needs defined as backlog. The second approach was based on the service life of the bridge (which is the minimum time before a major rehabilitation is performed). Only the bridges in the State Bridge Inventory (span greater than 20 feet) were included. Need for small bridges and culverts was estimated by updating the earlier estimate made by LTAP (2001). Table 2.69 summarizes the monetary needs for Indiana local bridges. 
Table 2.69: Summary of Local Bridge Needs (2006-2020) (\$Y Year 2002)

\begin{tabular}{|c|c|c|}
\hline & Bridges greater than 20 feet & Small Bridges and Culverts \\
\hline Backlog (as of 2006) & $\$ 456$ Million & $\$ 170$ Million \\
\hline $\begin{array}{c}\text { Total Needs including } \\
\text { backlog over the Study } \\
\text { Period (2006-2020) }\end{array}$ & $\$ 1.2$ Billion & $\$ 995$ Million \\
\hline
\end{tabular}

Based on Estimation by LTAP (2001)

\subsubsection{Assessment of Overhead Costs and Capacity Needs}

In order to determine the total needs for Indiana local agencies, it was necessary to take into account the "Overhead Costs". Overhead costs were referred to as the money needed for administrative support and infrastructure maintenance, not directly chargeable to a particular work element. In addition, an estimate was made of the cost for added capacity that can be expected to accommodate the increasing traffic. The following paragraphs present the estimation of these costs for Indiana local agencies during the study period.

\section{Overheads Costs}

Using the sample of eight counties, the percentage of the MVHA receipts used for administrative and operational expenses were determined for the years 1998 and 2000. An average range of percentages was then estimated and applied to derive the statewide average. Table 2.70 shows the percentages found for the sample of eight counties as well as the average percentages to be used.

To estimate the future revenue for the MVHA account, a 6-year average was used. Accordingly, the local share of MVHA receipts is expected to attain $\$ 4.75$ billion over the 15 -year period of study, representing approximately $\$ 317$ million of revenue each year. On the basis of the sample of eight counties, overhead costs represent approximately 39 to 47\% of MVHA revenues. Thus, the total overhead costs for the study period are estimated at $\$ 1.8$ to $\$ 2.25$ billion, as shown in Table 2.81 . 
Table 2.70: Local Overhead Costs as a Percentage of MVHA Receipts (sample of 8 counties) (\$ Year 2002)

\begin{tabular}{|c|c|c|c|c|c|}
\hline & & Administration & $\begin{array}{l}\text { Administrative } \\
\text { and } \\
\text { Undistributed } \\
\text { Expenses } \\
\end{array}$ & $\begin{array}{l}\text { MVHA } \\
\text { Receipts }\end{array}$ & $\%$ \\
\hline \multirow[t]{2}{*}{ Adams } & 1998 & 108,727 & 523,110 & $1,852,873$ & 34.10 \\
\hline & 2000 & 116,726 & 737,352 & $1,932,611$ & 44.19 \\
\hline \multirow[t]{2}{*}{ Fayette } & 1998 & 13,752 & 487,944 & $1,378,346$ & 36.40 \\
\hline & 2000 & 618,430 & 202,404 & $1,442,397$ & 56.91 \\
\hline \multirow[t]{2}{*}{ Fountain } & 1998 & 67,015 & 564,425 & $2,022,009$ & 31.23 \\
\hline & 2000 & 71,270 & 705,095 & $1,945,065$ & 39.91 \\
\hline \multirow[t]{2}{*}{ Floyd } & 1998 & 152,276 & 476,522 & $1,763,952$ & 35.65 \\
\hline & 2000 & 134,065 & 491,230 & $1,730,562$ & 36.13 \\
\hline \multirow[t]{2}{*}{ Hamilton } & 1998 & $1,118,238$ & 921,106 & $3,538,886$ & 57.63 \\
\hline & 2000 & $1,293,104$ & $1,201,507$ & $3,898,297$ & 63.99 \\
\hline \multirow[t]{2}{*}{ Lawrence } & 1998 & 136,454 & 43,896 & $1,961,487$ & 9.19 \\
\hline & 2000 & 167,651 & 45,525 & $1,969,342$ & 10.82 \\
\hline \multirow[t]{2}{*}{ Pike } & 1998 & 115,214 & 616,207 & $1,257,627$ & 58.16 \\
\hline & 2000 & 124,364 & 705,008 & $1,358,550$ & 61.05 \\
\hline \multirow[t]{2}{*}{ White } & 1998 & 65,921 & 822,228 & $2,210,528$ & 40.18 \\
\hline & 2000 & 115,602 & $1,080,072$ & $2,346,665$ & 50.95 \\
\hline \multirow{2}{*}{$\begin{array}{l}\text { Total } \\
\text { Sample }\end{array}$} & 1998 & $1,777,599$ & $4,455,442$ & $15,985,713$ & 38.99 \\
\hline & 2000 & $2,641,214$ & $5,168,196$ & $16,623,492$ & 46.98 \\
\hline
\end{tabular}

\section{Capacity Needs}

To determine the capacity needs, a file provided by INDOT, the "Directory of Local Community Highway Projects", was used. The file reports the local highway projects in Indiana planned from 1999 to 2016. An approximate cost for each project is also estimated in the same file. The projects for the years falling in the study period (2006-2016) were considered in the present analysis and the capacity needs for the 4 remaining years (2016-2020) were extrapolated using the 
average annual need. The directory includes projects such as added travel lanes, pavement and bridge replacements, rehabilitations and constructions, intersection improvements, traffic sign replacements, etc. A summary of the file can be found in Appendix L. The total need for the study period was estimated at approximately $\$ 2.2$ billion. The results are shown on Table 2.71 .

Table 2.71: Estimation of Capacity Needs for Indiana Local Roads (\$ Year 2002)

\begin{tabular}{|c|c|}
\hline Total Estimated Needs for the Period 2006-2016 & $\$ 1,705,659$ \\
\hline Average Annual Need & $\$ 129,148$ \\
\hline Total Needs for the Study Period (2006-2020) & $\$ 2,222,250$ \\
\hline
\end{tabular}

\subsection{Total Needs for the State and Local Highway Network}

\section{State Highway Needs Summary}

The total 15-year (2006-2020) state highway need is estimated to be in range of $\$ 29.8$ to $\$$ 30.2 billion (2002\$), as shown in Tables 2.72. The estimates include the pavements, capacity and safety needs as well as the preservation needs for the new infrastructure and the needs for bridges. The need represents engineering needs and not projections of historical expenditures.

Table 2.72: 15-yr State Highway Needs (2006-2020)

\begin{tabular}{|c|c|}
\hline Category & Need (\$ Year 2002, in billion) \\
\hline Pavement and Safety & $\$ 17.1$ to $\$ 17.3$ \\
\hline $\begin{array}{l}\text { Capacity } \\
\text { (Includes all Interchanges, New Road, and Median } \\
\text { Construction) }\end{array}$ & $\$ 4.8$ \\
\hline $\begin{array}{l}\text { Preservation of New Infrastructure } \\
\text { (Pavement Preservation of Added Lanes) }\end{array}$ & $\$ 0.074$ to $\$ 0.20$ \\
\hline $\begin{array}{l}\text { Bridge Needs } \\
\text { (New Bridges and their Preservation) }\end{array}$ & $\$ 1.3$ \\
\hline Subtotal 15 yr Infrastructure Needs & $\$ 23.3$ to $\$ 23.6$ \\
\hline Overhead Costs & $\$ 6.5$ to $\$ 6.6$ \\
\hline Total 15 yr State Highway Needs & $\$ 29.8$ to $\$ 30.2$ \\
\hline Total State Highway Needs per year & $\$ 1.98$ to $\$ 2.01$ \\
\hline
\end{tabular}




\section{Local Highway Needs}

The total 15-year (2006-2020) local highway need in Indiana is estimated to be in a range of $\$ 28.1$ billion to $\$ 30.4$ billion (2002\$), as shown in Table 2.73. Needs estimated include the preservation needs for both paved and unpaved roads, the capacity and safety needs as well as the need for bridges and culverts.

Table 2.73: 15-yr Local Highway Needs (2006-2020)

\begin{tabular}{|l|c||}
\hline \multicolumn{1}{|c|}{ Category } & Needs (\$ Year 2002, in billion) \\
\hline \hline Pavement Preservation & $\$ 21.6-\$ 23.4$ \\
\hline \hline Bridges and Culverts & $\$ 1.96$ \\
\hline \hline Safety & $\$ 0.56$ \\
\hline \hline Capacity & $\$ 2.2$ \\
\hline Overhead Costs & $\$ 1.8-\$ 2.2$ \\
\hline \hline Total 15- yr Local Highway Needs & $\$ 28.1-\$ 30.4$ \\
\hline \hline Total Local Highway Needs per year & $\$ 1.9-\$ 2.03$ \\
\hline \hline
\end{tabular}




\section{CHAPTER 3: ESTIMATION OF REVENUES}

The preceding chapter estimated the 15-year highway needs for both the state and local highway systems in Indian. The intent of this chapter is to estimate the existing and potential resources available to finance those needs. In order to estimate revenues expected for the state and local highway network, the existing financing structure and the historical trends in state highway revenue were examined. In addition, highway revenue forecasting developed earlier (Varma et al. 1991) was updated and used. By comparing needs with projected revenues the expected revenue gaps were identified under different scenarios.

\subsection{Estimation of State Highway Revenues}

The data sources for the analysis of state highway revenue include: "Indiana Handbook of Taxes, Revenues, and Appropriations (Fiscal Year 2003 Edition)," by the Indiana Legislative Services Agency, Office of Fiscal and Management Analysis; INDOT Annual Reports; historical revenue and budget information obtained from the INDOT Budget and Fiscal Management Division; and the JTRP report entitled, "An Evaluation of Innovative Transportation Financing Techniques for Indiana," by Drike et al. (2002).

\subsubsection{State Highway Funding}

The state generates funds for highway infrastructure projects from both user fees and nonuser fees. User fees include vehicle license and registration fees and motor fuel taxes. Motor fuel taxes include:

- Gasoline Tax;

- Special Fuel Tax;

- Motor Carrier Fuel Use Tax (MCFUT);

- Motor Carrier Surcharge Tax; and

- Alternative Fuel Decals.

The four largest highway revenue sources for the state are gasoline/gasohol tax; diesel and special fuels tax; vehicle registration, title and license fees; and the motor carrier surtax. The contributions of these user taxes are illustrated in Figure 3.1. Gasoline/gasohol taxes account for the largest share of the state generated highway revenue, representing $44.4 \%$ of the total highway revenue in FY 2002. The percentage of the total highway revenue that each user fee represents is 
illustrated in Figure 3.2. Revenue from these user fees are shared among the state and local governments through a state highway revenue distribution formula (INDOT 2003c).

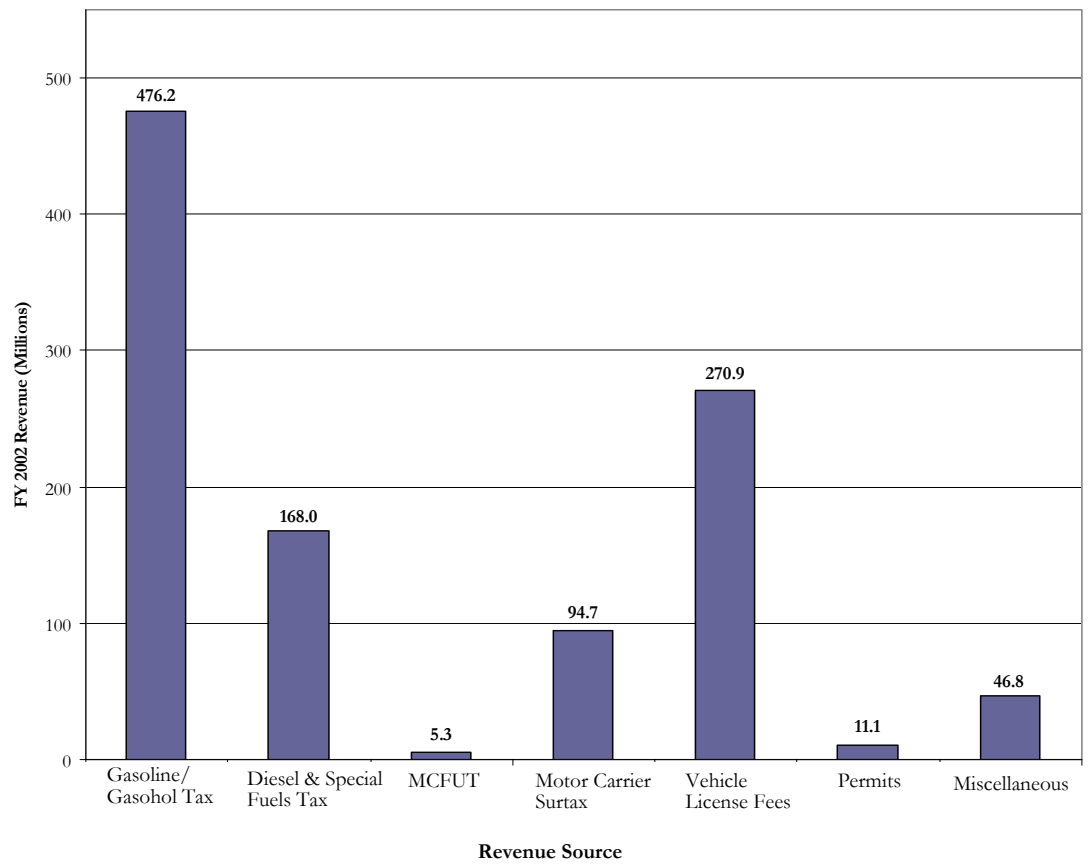

Figure 3.1 State Generated Highway Revenue Sources FY 2002 (INDOT 2003c) 


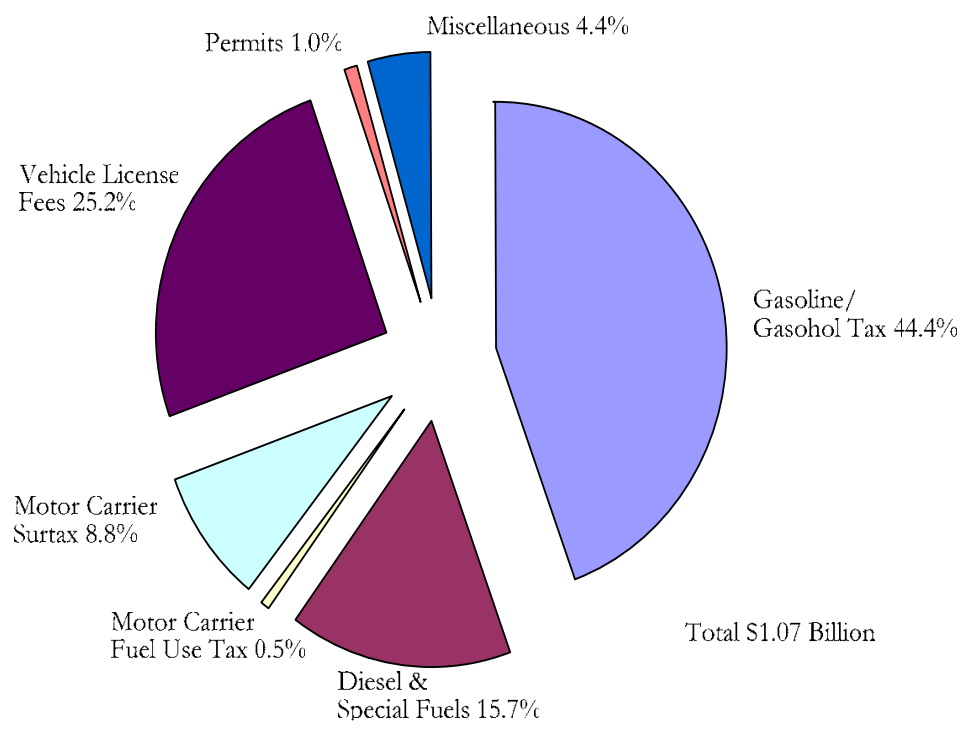

Figure 3.2 Relative Contributions from Revenue Sources FY 2002 (INDOT 2003)

In fiscal year 2002, fuel tax revenues accounted for $69 \%$ of the total state highway revenue while non-fuel related revenues accounted for 31\% of total revenues (Table 3.1). Miscellaneous revenue, which accounts for approximately $4.4 \%$ of the total revenue, is a combination of various sources. Each miscellaneous source in the total grouping may have a different share guaranteed for INDOT. The revenue from miscellaneous sources varies depending on the make-up of the grouping of miscellaneous items occurring in a year. An example of a miscellaneous revenue source is funds from auctions of abandoned cars. Any residual revenue after towing and auctioning an abandoned car goes to the Motor Vehicle Highway Account (MVHA) which is discussed later in this chapter.

For fiscal year 2002, total state highway revenue for INDOT was $\$ 549.5$ million, while $\$ 379.1$ million was provided to counties, cities, and towns (INDOT 2003a). Non-user sources of revenue for transportation infrastructure include bonds and the state's general fund. 
Table 3.1 State Generated Highway Revenue FY 2002 (Millions)

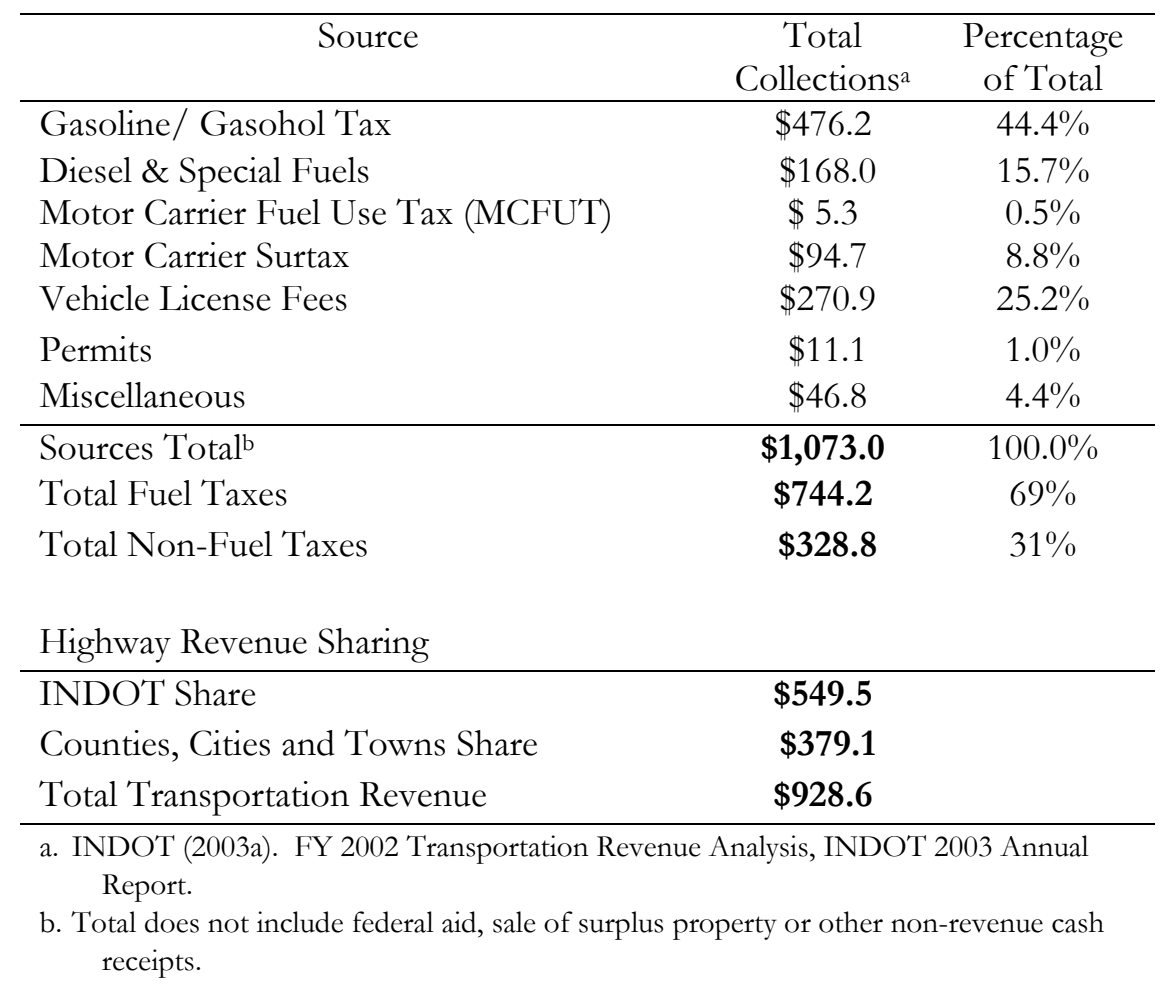

All highway revenue obtained does not go directly to highway infrastructure projects. There are several "net" expenses that are paid by state generated highway revenue such as: State Police, Bureau of Motor Vehicles, Department of Revenue, the Motor Carrier Regulation Fund, Safety and the General Fund. The balance remaining is what is allocated to state and local highway agencies. The general fund covers approximately fifty percent of the gross state police expense. Table 3.2 presents the total "net" expenses of 144.4 million dollars for fiscal year 2002 representing $13.46 \%$ of the total revenue of 1,073 million dollars. The maximum amounts of "net" expense, such as for the Bureau of Motor Vehicles and the State Police, are appropriated by the State Legislature every two years. 
Table 3.2 State Highway Net Expenses FY 2002 (Millions)

\begin{tabular}{lcc} 
Net Expenses & Expense & $\begin{array}{c}\text { Percentage of } \\
\text { Total Expenses }\end{array}$ \\
\hline State Police & $\$ 55.3$ & $38.3 \%$ \\
Bureau of Motor Vehicles & $\$ 40.9$ & $28.3 \%$ \\
General Fund & $\$ 30.0$ & $20.8 \%$ \\
Department of Revenue & $\$ 8.6$ & $6.0 \%$ \\
Motor Carrier Regulation Fund & $\$ 7.9$ & $5.5 \%$ \\
Safety & $\$ 1.6$ & $1.1 \%$ \\
Miscellaneous & $\$ 0.1$ & $0.1 \%$ \\
\hline Total & $\$ \mathbf{1 4 4 . 4}$ & $100.0 \%$
\end{tabular}

INDOT, 2003a

Typically, the largest "net" expenses for INDOT are the State Police and the Bureau of Motor Vehicles. These represented 38.3\% and 28.3\%, respectively of total net expenses in 2002 . The General Fund expense of $\$ 30$ million is not a typical annual expense. In the nine-year period between 1995 and 2003, it was only in 2002 that the General Fund constituted a "net" expense to the state highway funds. This was a special case, because Indiana, as well as many other states, was faced with large state budget deficits.

After the net expenses are subtracted from the highway revenue, the remaining funds are deposited into the MVHA, where it is then disbursed between the state (INDOT) and the local agencies using a 53 percent to 47 percent ratio. The INDOT funds are held in the State Highway Fund (SHF) while the local agency funds are further disbursed among the counties, cities, and towns based on established formulas that depend on population, number of registered vehicles, and mileage of roadway. The SHF receives revenue directly from gasoline tax revenue and indirectly from other vehicle highway accounts. Figure 3.3 illustrates how the funds are deposited to the SHF. 


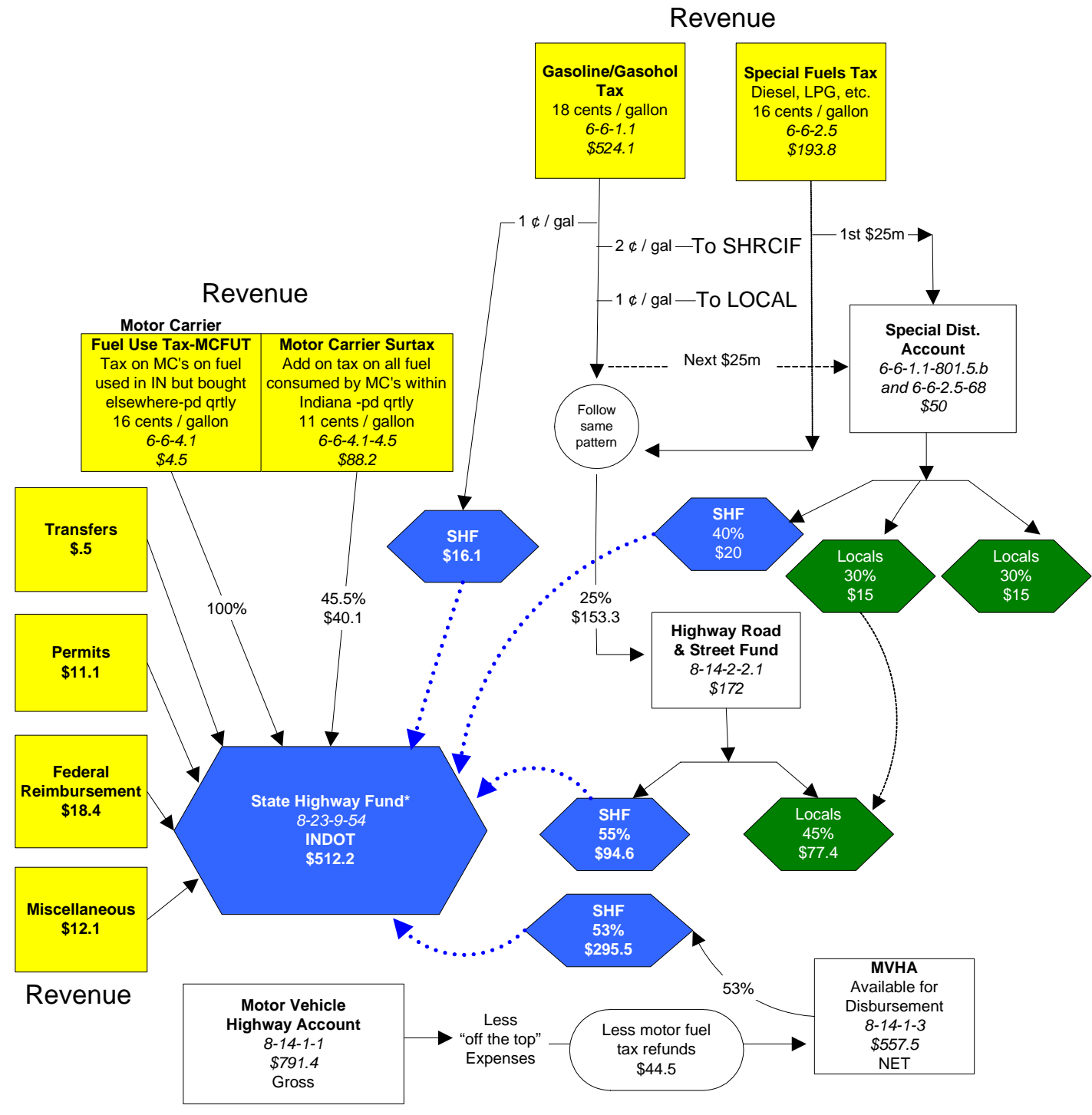

Figure 3.3 State Highway Fund Revenue Flowchart (INDOT, 2003g)

(Dollar amounts shown are in millions)

In January 2003, the gasoline tax was increased by three cents per gallon and distributed as follows: one cent per gallon goes directly to the SHF, one cent per gallon goes directly to local distribution and the distribution to the State Highway Road Construction and Improvement Fund (SHRCIF) was increased from one cent per gallon to two cents per gallon. SHRCIF is a fund created by the Indiana legislature in 1988 to be used for bond repayment. SHRCIF is discussed later in this chapter. Forty percent of funds going to the Special Distribution Account are directed to the SHF. The funds going to the Special Distribution Account are fixed at $\$ 50$ million, $\$ 25$ million from the gasoline/gasohol tax and $\$ 25$ million from the special fuels tax. The SHF also obtains funds from 
the Net MVHA and the MCFUT. As previously stated, 53\% of funds in the Net MVHA go to the SHF. All revenue in the MCFUT Fund, $\$ 4.5$ million in FY 2003, is deposited to the SHF.

Special permits can be issued to allow vehicles in excess of maximum size or weight limits to travel on Indiana highways. INDOT administers this fund, and revenues are distributed to the SHF (ILSA 2003). In fiscal year 2003, permits accounted for 11.1 million dollars in revenue as illustrated in Figure 3.3.

\subsubsection{Highway Road and Street Fund}

The Highway, Road, and Street Fund (HRSF) receives 25\% of the gasoline tax, 25\% of the special fuel tax, increased fees under Indiana Code IC 9-1-1, and 25\% of the alternative fuel use decal revenue, as illustrated in Figure 3.4. Money in the HRSF is distributed monthly by the Auditor of State to the respective units of government as follows:

- State Highway Fund (SHF)- INDOT receives 55\% of the amount in the HRSF plus all revenues from the Motor Carrier Fuel Tax and 45.5\% of the Motor Carrier Surtax.

- Local Road and Street Fund (LRSF) - receives 45\% of the amount in the HRSF. This fund is distributed to each county based on county passenger car registrations as compared to total passenger car registrations. The suballocation of the funds is described in the next section.

The HRSF funds distributed to the SHF must be appropriated for their use. The HRSF funds that go to the LRSF are not required to be appropriated. The LRSF can be used for the following purposes:

1. Engineering and land acquisition;

2. Construction, maintenance, resurfacing, restoration, or rehabilitation;

3. Payment of principal and interest on bonds sold primarily to finance road, street, or thoroughfare projects; or

4. Any local costs required to undertake a recreational or reservoir road project.

Counties having population of 50,000 or more share the HRSF revenues based on the following criteria:

- $60 \%$ based on population of unit to total population of the county.

- $40 \%$ on ratio of unit's street mileage to total road mileage in the county.

For counties having population of 50,000 or less, the formula is as follows:

- $20 \%$ based on population of unit to total population of the county. 
- $80 \%$ on ratio of unit's street mileage to total road mileage in the county.

\subsubsection{Motor Vehicle Highway Account}

The revenue sources for the MVHA, its expenses, and distribution to the state and local jurisdictions for the state fiscal year 2003 (July 1, 2002 to June 30, 2003) are illustrated in Figure 3.4. The MVHA receives $75 \%$ of the gasoline tax and $75 \%$ of special fuel tax collections (less administrative expenses and the first $\$ 25,000,000$ collected from each tax), motor vehicle registration fees, and $45.5 \%$ of the Motor Carrier Surtax. The net amount in the account is distributed, with 47\% of the funds going to local jurisdictions and 53\% going to INDOT. Revenues of 791.4 million dollars went into the Gross MVHA account during fiscal year 2003. This total included 88.2 million from the Motor Carrier Surtax; 80.1 million from the International Registration Plan (IRP); 4.7 million from miscellaneous revenue such as funds from auto dealers; 75.6 million from reimbursements; 3.6 million from State Court Fees, which are fixed by state statute; 460 million from Gasoline/Gasohol, following the required distributions to other accounts; and 127.3 million from Vehicle License and Registration Fees. Money in the MVHA is distributed monthly by the Auditor

of State to the respective units of government. Tables 3.3 and 3.4 present trends in MVHA revenue amounts by source. 


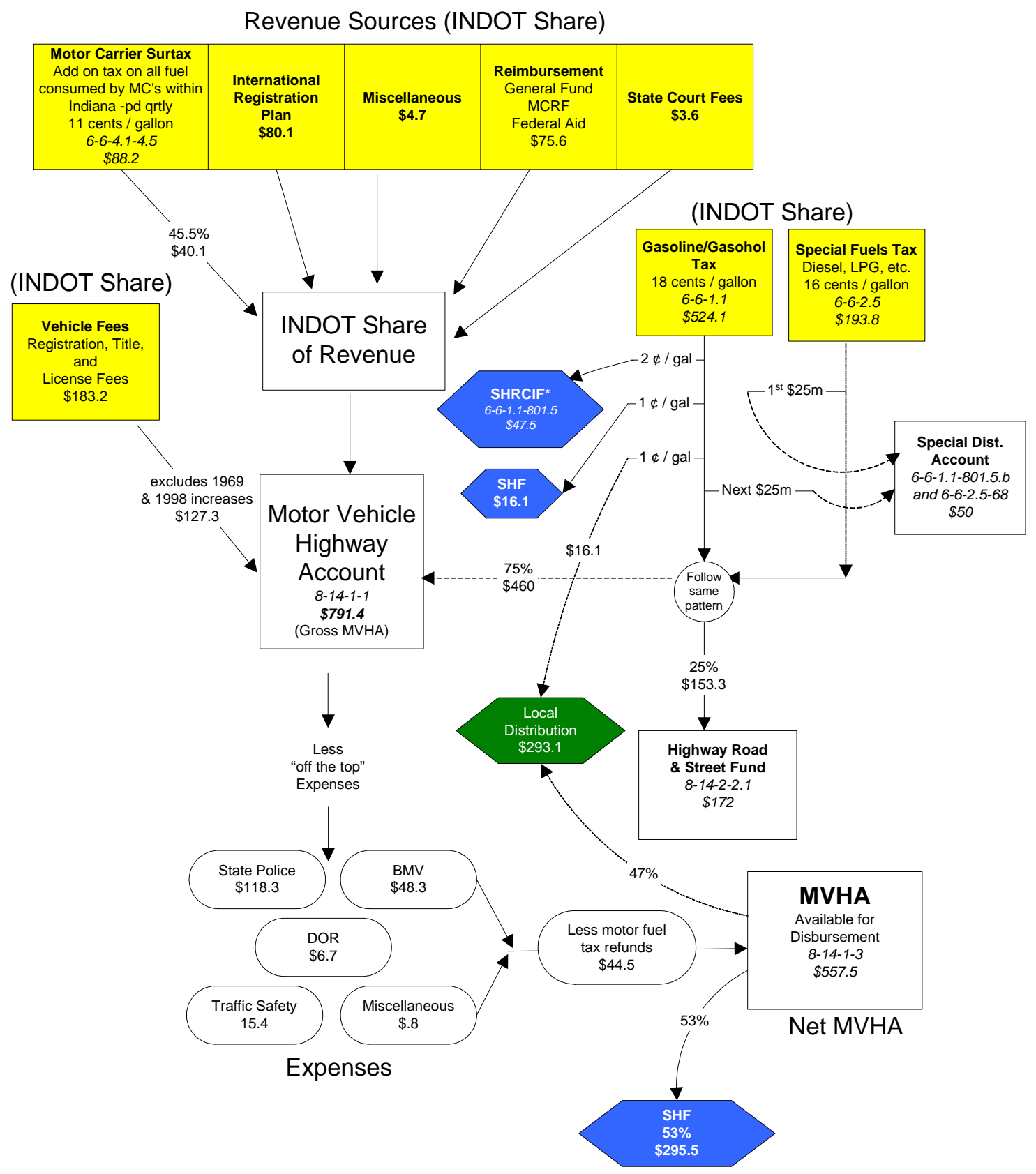

Figure 3.4 Motor Vehicle Highway Account Funding Flowchart FY 2003 (INDOT, 2003g) (Dollar amounts shown are in millions)

As shown in Figure 3.4, 47\% of MVHA fund is distributed to local jurisdictions. These funds are sub allocated to cities, towns, and counties based on the following criteria:

- $15 \%$ to cities and towns, based on the population of city compared to total city populations. 
- $32 \%$ to counties, using the following formula: $5 \%$ equally to all counties, $65 \%$ on the basis of actual county road miles to total county road miles, and 30\% based on motor vehicle registrations compared to total motor vehicle registrations statewide.

The allowable uses of the MVHA funds distributed to counties, cities, and towns as described in the 2003 Indiana Handbook of Taxes, are shown below:

1. Construction, reconstruction, and maintenance;

2. Purchase, rental, and repair of highway equipment;

3. Painting of bridges;

4. Land acquisition;

5. Construction of storage buildings; and

6. Fuel oil and supplies.

The allowable uses of the MVHA distribution to cities and towns include:

1. Construction, reconstruction, and maintenance;

2. Oiling, sprinkling, snow removal, weed and tree cutting, and cleaning of highways;

3. Purchase or lease of highway construction equipment;

4. Traffic signs and signals;

5. Safety zones and devices;

6. Traffic policing and safety (however, a unit may not spend more than $10 \%$ of the distribution for this purpose unless the population is less than 5,000, which allows spending $15 \%)$; and

7. Painting of structures.

MVHA funds may be appropriated to INDOT for the purpose of maintaining a sufficient working balance in its accounts established primarily to facilitate the matching of federal and local money for highway projects. 
Table 3.3 MVHA Historical Revenue 1995-1999 (Current \$)

\begin{tabular}{|c|c|c|c|c|c|}
\hline \multirow[b]{2}{*}{ Fuel Tax Revenue } & \multicolumn{5}{|c|}{ 1995-1999(Millions) } \\
\hline & FY95 & FY96 & FY97 & FY98 & FY99 \\
\hline Gasoline & 282.8 & 288.4 & 291.8 & 300.0 & 306.8 \\
\hline Special Fuels & 90.5 & 109.1 & 114.4 & 122.2 & 127.7 \\
\hline Motor Carrier Surtax & 35.1 & 38.3 & 28.9 & 46.0 & 43.7 \\
\hline Fuels tax refunds & $(38.0)$ & $(34.5)$ & $(29.8)$ & $(39.0)$ & $(34.4)$ \\
\hline Net Motor Fuel Taxes & 370.5 & 401.3 & 408.5 & 429.2 & 443.8 \\
\hline \multicolumn{6}{|l|}{$\begin{array}{l}\text { Fees, Reimbursements, Permits and Other } \\
\text { Sources }\end{array}$} \\
\hline Vehicle License Fees & 103.9 & 109.9 & 112.2 & 115.0 & 117.6 \\
\hline International Registration Plan & 65.4 & 67.4 & 81.4 & 65.4 & 74.8 \\
\hline General Fund Reimbursement Police & 20.7 & 44.7 & 52.7 & 54.1 & 56.9 \\
\hline Special Fuel Reimbursement Police & 0.0 & 3.1 & 0.0 & 0.0 & 0.0 \\
\hline MCRF Reimbursement Police Only & 3.2 & 4.2 & 4.7 & 4.4 & 4.6 \\
\hline Traffic Safety, Federal Reimbursement & 4.0 & 5.4 & 4.2 & 4.5 & 3.7 \\
\hline Total Revenue & 597.1 & 650.1 & 678.5 & 690.6 & 716.1 \\
\hline \multicolumn{6}{|l|}{ Disbursements } \\
\hline Bureau of Motor Vehicles & 36.2 & 28.1 & 29.7 & 44.7 & 34.6 \\
\hline Indiana State Police & 82.5 & 101.3 & 106.7 & 113.9 & 117.1 \\
\hline Dept. of Revenue (Fuel Tax Division) & 3.4 & 4.7 & 5.5 & 5.4 & 5.1 \\
\hline Traffic Safety Programs & 4.1 & 6.0 & 5.2 & 4.4 & 4.3 \\
\hline Miscellaneous & 0.8 & 0.8 & 0.7 & 0.7 & 0.0 \\
\hline Auditor Adjustments & 13.2 & $(0.7)$ & 1.8 & $(0.5)$ & 0.4 \\
\hline Year 2000 Computer Fund & 0.0 & 0.0 & 0.0 & 0.0 & 3.6 \\
\hline Total Disbursements & 140.7 & 141.0 & 149.6 & 168.6 & 165.1 \\
\hline Revenue Available for Distribution & 456.4 & 509.8 & 528.8 & 522.0 & 551.0 \\
\hline $53 \%$ to State Highway Fund & 241.9 & 270.2 & 280.3 & 276.7 & 292.0 \\
\hline
\end{tabular}

Source: Jackson (2003). 
Table 3.4 MVHA Historical Revenue 2000-2003 (Current \$)

\begin{tabular}{|c|c|c|c|c|}
\hline \multirow[b]{2}{*}{ Fuel Tax Revenue } & \multicolumn{4}{|c|}{ 2000-2003 (Millions) } \\
\hline & FY00 & FY01 & FY02 & FY03 \\
\hline Gasoline & 311.4 & 324.6 & 314.9 & 314.6 \\
\hline Special Fuels & 143.0 & 123.4 & 135.8 & 145.4 \\
\hline Motor Carrier Surtax & 41.1 & 39.4 & 43.4 & 40.1 \\
\hline Fuels tax refunds & $(34.6)$ & $(33.8)$ & $(39.1)$ & $(44.5)$ \\
\hline Net Motor Fuel Taxes & 460.9 & 453.6 & 454.9 & 455.6 \\
\hline \multicolumn{5}{|l|}{$\begin{array}{l}\text { Fees, Reimbursements, Permits and Other } \\
\text { Sources }\end{array}$} \\
\hline Vehicle License Fees & 121.1 & 122.6 & 123.3 & 127.3 \\
\hline International Registration Plan & 91.7 & 61.5 & 93.2 & 80.1 \\
\hline General Fund Reimbursement Police & 60.5 & 61.3 & 55.0 & 52.3 \\
\hline Special Fuel Reimbursement Police & 0.0 & 0.0 & 0.0 & 0.0 \\
\hline MCRF Reimbursement Police Only & 5.1 & 5.2 & 3.1 & 5.2 \\
\hline Traffic Safety, Federal Reimbursement & 4.6 & 5.1 & 10.6 & 13.1 \\
\hline Total Revenue & 760.9 & 726.3 & 756.3 & 746.9 \\
\hline \multicolumn{5}{|l|}{ Disbursements } \\
\hline Bureau of Motor Vehicles & 40.3 & 40.9 & 40.9 & 48.3 \\
\hline Indiana State Police & 125.8 & 128.9 & 113.5 & 118.3 \\
\hline Dept. of Revenue (Fuel Tax Division) & 6.4 & 5.8 & 8.6 & 6.7 \\
\hline Traffic Safety Programs & 5.9 & 6.2 & 9.2 & 15.4 \\
\hline Miscellaneous & 0.2 & 0.0 & 3.4 & 0.8 \\
\hline Auditor Adjustments & 0.7 & 0.4 & 0.0 & 0.0 \\
\hline Year 2000 Computer Fund & 6.0 & 0.0 & 0.0 & 0.0 \\
\hline Total Disbursements & 185.3 & 182.2 & 175.7 & 189.4 \\
\hline Revenue Available for Distribution & 575.6 & 544.1 & 580.6 & 557.5 \\
\hline $53 \%$ to State Highway Fund & 305.1 & 288.4 & 307.7 & 295.5 \\
\hline
\end{tabular}

\subsubsection{Vehicle Registration and Licensing Fees}

The Bureau of Motor Vehicles (BMV) is responsible for collecting the license and vehicle registration fees. The revenue for vehicle registration and title fees is distributed to the MVHA. The revenue base for vehicle registration and title fees are the owners of motor vehicles who pay an annual registration fee, and all registrations are renewed between January and October of each year. On January 1, 2002, new fees for vehicle registration and titling became effective as a result of action taken by the Bureau of Motor Vehicles Commission (BMVC) and the General Assembly during the 2001 legislative session., and the vehicle registration fees increased by four dollars. 
The BMVC is permitted to retain a service charge of $\$ 2.75$ from the registration fee for each registration processed. They also retain $\$ 2.00$ from each title fee and duplicate fee, $\$ 7.00$ from the delinquent title fee, and $\$ 2.00$ from the transfer fee. All fees go to the MVHA, except for the increased fees enacted in 1969 and $\$ 5.00$ for each motorcycle which goes to the Motorcycle Operators Safety Education Fund (Acct. 212-700). Fifty-five percent of revenue from fees enacted in 1969 is deposited into the State Highway Fund and 45\% to the Local Road and Street Fund.

\subsubsection{Description of Tax Types}

\section{Gasoline/Gasohol Tax}

According to the U.S. Department of Energy Information Administration, 28 percent of the retail price of one gallon of gasoline in 1999 and 2000 represented federal, state and other local taxes (Puentes and Prince, 2003). In the state of Indiana approximately 3.1 billion gallons of gasoline and 1.3 billion gallons of diesel fuel are sold per year (INDOT, 2003). In FY 2002, Indiana state fuel taxes represented $69 \%$ of the state highway revenue for use on highway projects (INDOT, 2003a). A chronological history of the fuel tax rates in Indiana is provided in Table 3.5. Fuel tax rates can only be changed by state legislative action. The gasoline tax rate remained at 15 cents per gallon between 1988 and December 2002.

Table 3.5 History of Indiana Fuel Tax Rates 1923 - 2003 (cents/gallon)

\begin{tabular}{cccc}
\hline Year & $\begin{array}{c}\text { Gasoline } \\
\text { Tax Rate }\end{array}$ & $\begin{array}{c}\text { Diesel Tax } \\
\text { Rate }\end{array}$ & $\begin{array}{c}\text { Diesel } \\
\text { Surtax }\end{array}$ \\
\hline 1923 & 2.0 & 0.0 & 0.0 \\
\hline 1925 & 3.0 & 0.0 & 0.0 \\
1929 & 4.0 & 0.0 & 0.0 \\
1943 & 4.0 & 4.0 & 0.0 \\
1957 & 6.0 & 6.0 & 0.0 \\
1969 & 8.0 & 8.0 & 0.0 \\
1980 & 8.5 & 8.5 & 0.0 \\
1981 & 10.5 & 10.5 & 0.0 \\
1982 & 11.1 & 11.1 & 0.0 \\
1985 & 14.0 & 15.0 & 8.0 \\
1988 & 15.0 & 16.0 & 11.0 \\
2003 & 18.0 & 16.0 & 11.0 \\
\hline
\end{tabular}


The gasoline tax rate in Indiana is lower than the national average and when compared to its four neighboring states, Indiana has the second lowest tax rate as shown in Table 3.6.

Table 3.6 Fuel Tax Rates in Indiana and Neighboring States (cents/gallon)

\begin{tabular}{cccccccc} 
Source & Indiana & Illinois & Kentucky & Michigan & Ohio & Federal & US Average \\
\hline Gasoline & 18.0 & 19.8 & 16.4 & 19.0 & 22.0 & 18.4 & 20.4 \\
Diesel & $27.0^{\mathrm{a}}$ & 22.3 & 13.4 & 15.0 & 22.0 & 24.4 & 23.9 \\
\hline
\end{tabular}

a. Indiana diesel tax as shown includes a 16 cents/gallon diesel tax and an 11 cents/gallon diesel surtax. Source: INDOT, (2003c).

The motor fuel tax rate for Indiana was raised to 18 cents/gallon in January 2003, but it is still below the inflation-adjusted tax rate. The Indiana diesel and diesel surtax rates still remain at their 1988 levels. The 1988 gasoline tax rate, adjusted to 2004 cents, is calculated to be 22.1 cents/gallon, which represents a four cents/gallon gas tax increase. A summary of how the various motor fuel taxes could be appropriately adjusted for inflation is illustrated in Table 3.7.

Table 3.7 Inflation Impact of Highway Revenue (Millions)

\begin{tabular}{cccccc}
\hline \multicolumn{2}{c}{ Gasoline } & Tax Rate & \multicolumn{2}{c}{ Diesel Tax Rate } & \multicolumn{2}{c}{ Diesel Surtax } \\
\hline \$Year & $\$$ Year & \$Year & \$Year & \$Year & \$ Year \\
1988 & 2002 & 1988 & 2002 & 1988 & 2002 \\
\hline 15.0 & 20.8 & 16.0 & 22.2 & 11.0 & 20.5 \\
All tax rates are shown in cents/gallon. & & &
\end{tabular}

A sample calculation of the 1988 gasoline tax (15 cents per gallons) adjusted to year 2002 constant dollars (20.8 cents per gallon) is illustrated in this section. The CPI values used in the analysis were discussed in Chapter 2.

$$
\begin{aligned}
\text { Tax Rate }_{2002} & =(\text { Tax Rate } \\
1998 & \left.* \mathrm{CPI}_{2002}\right) / \mathrm{CPI}_{1998} \\
\text { Tax Rate } 2002 & =(15 \text { cents per gallon } * 147.9) / 106.6 \\
\text { Tax Rate }_{2002} & =20.8 \text { cents per gallon }
\end{aligned}
$$

\section{Gasoline Tax Revenue Distribution}

Licensed distributors make monthly payments based on invoiced gallons received minus a $1.6 \%$ distributor allowance to cover evaporation, shrinkage, losses, and collection expenses. The gasoline tax revenue is administered by a Special Tax Division of the Department of Revenue and is distributed, as illustrated in Figure 3.5, and described here: 
- $1 / 9$ of the total tax collected ( 2 cents per gallon of the total 18 cents per gallon), is deposited to the State Highway Road Construction and Improvement Fund (SHRCIF) and is used to pay debt-service (bonds).

- $1 / 18$ ( 1 cent per gallon) of total tax collected is deposited into the SHF (INDOT).

- $1 / 18$ of the total tax collected is deposited into the Gas Tax Fund and is distributed through the MVHA formula.

- Twenty-five million dollars is deposited into the Special Distribution Account and is distributed $60 \%$ to local units and $40 \%$ to the SHF.

- Of the remaining gasoline tax funds, $75 \%$ is deposited to the MVHA, $25 \%$ is deposited into the Highway Road and Street Fund. The funds that are deposited into the Highway Road and Street Fund are then distributed between the SHF and the Local Road and Street Account at a 55\% to $45 \%$ ratio. 


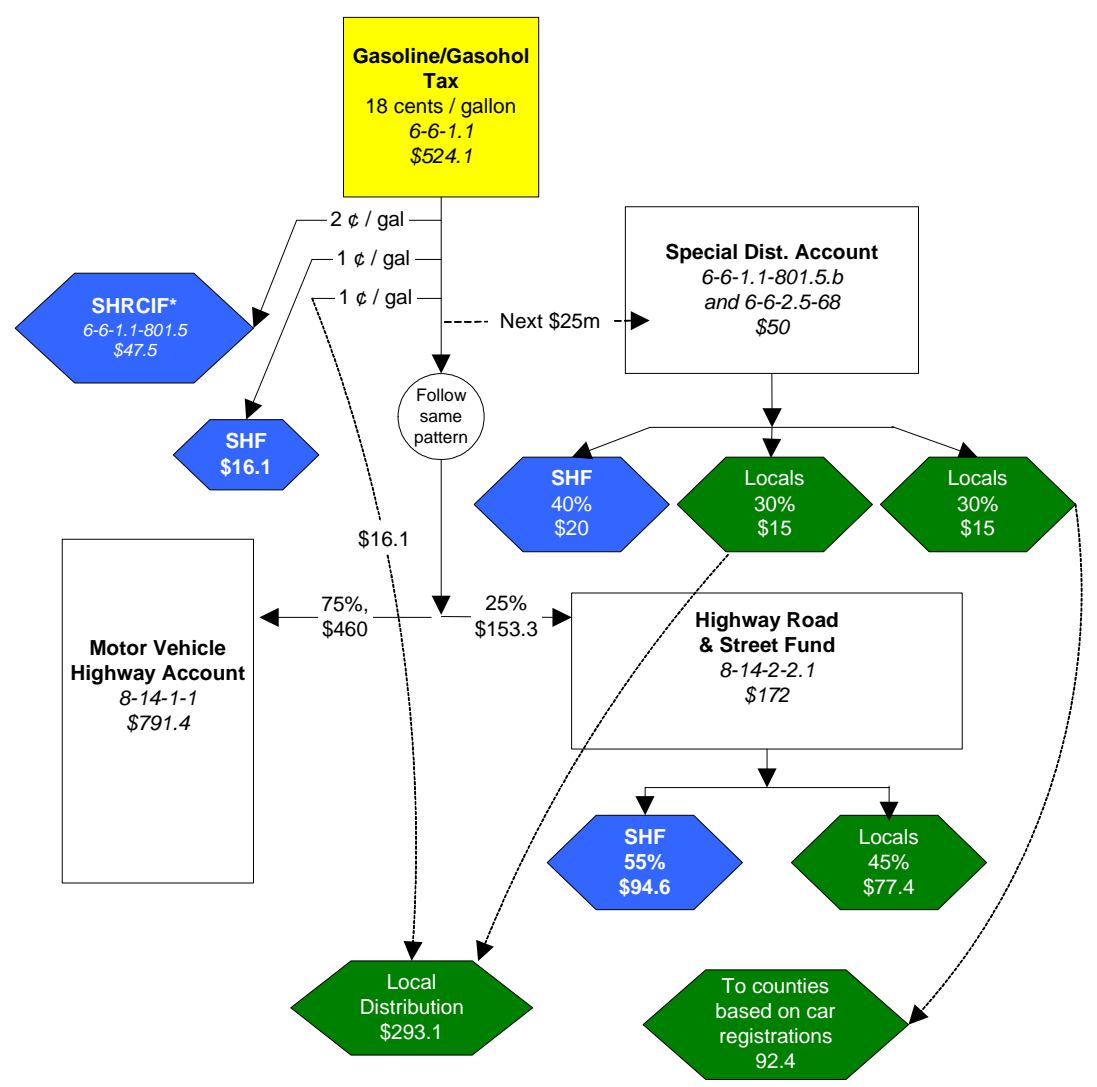

Figure 3.5 Gasoline Tax Distribution Flowchart FY 2003 (INDOT 2003g) (Dollar amounts shown are in millions)

Gasoline tax exemptions are applicable to the following persons, agencies and jurisdictions:

1. Gasoline sold to persons who have been issued an exemption permit and who operate either an airport where gasoline is sold for the exclusive purpose of propelling aircraft engines or a marine facility, except a taxable marine facility where gasoline is sold for the exclusive purpose of propelling motorboat engines.

2. Gasoline exported from Indiana to another state, territory, foreign country, or other jurisdiction.

3. Gasoline sold to the U.S. Government, an agency of the U.S. Government, or an instrumentality of the U.S. Government.

4. Gasoline used by a licensed distributor for any purpose other than the generation of power for the propulsion of motor vehicles upon public highways.

5. Gasoline received by a licensed distributor and thereafter lost or destroyed except by evaporation shrinkage or unknown cause while the distributor is still the owner thereof as a 
result of theft, leakage, fire, accident, explosion, lightning, flood, storm, act of war, public enemy, or other like cause.

6. Gasoline sold to a post exchange or other concessionaire on a federal reservation within Indiana.

Some examples of cases where a refund on gasoline tax paid can be requested include gasoline purchased or used for operating stationary gasoline engines, equipment mounted on motor vehicles whether or not operated by the engine propelling the motor vehicle, a tractor used for agricultural purposes, motorboats or aircraft, except propelling motor vehicles operated in whole or in part on an Indiana public highway, and gasoline in excess of 100 gallons which is lost or destroyed. Consumers may claim a gasoline tax refund by filing a refund claim within three years from the date the gasoline was purchased.

\section{Marine Fuel Tax}

The marine fuel tax is paid by licensed gasoline distributors who are the first to receive gasoline in the state. The tax is added to the selling price. The tax base is the total gallons of gasoline sold to a taxable marine facility defined as a marina located on an Indiana lake. The rate of tax per gallon of gasoline sold is 18 cents. Licensed distributors make monthly payments based on invoiced gallons received minus a $1.6 \%$ allowance to cover evaporation, shrinkage, losses, and collection expenses. Information reports and tax payments are due by the 20th day of each month. The total amount of gasoline sold to taxable marine facilities during the month is identified on the Distributor's Monthly Report. The total tax paid each month as a result of these sales is then transferred into the Fish and Wildlife Fund of the Department of Natural Resources. A tax exemption is applicable when gasoline is sold to a person who has been issued an exemption permit and who operates a marine facility, except a taxable marine facility, and sells gasoline at that facility for the exclusive purpose of propelling motorboat engines.

\section{Motor Carrier Fuel Use Tax}

This tax is applicable to a carrier who operates or causes to be operated a commercial motor vehicle on any highway in Indiana. The tax applies to the total amount of motor fuel consumed by commercial motor vehicles operated by a carrier in its operations on highways in Indiana. The tax imposed does not apply to that portion of motor fuel used to propel equipment mounted on a motor vehicle having a common reservoir for locomotion on the highway and the operation of such equipment. The tax rate per gallon is 16 cents. The tax is paid quarterly by the carrier on or before the last day of the month immediately following the quarter. The tax is based on the amount of fuel consumed while traveling in Indiana. The amount of motor fuel consumed by a carrier is the total amount of motor fuel consumed in its entire operations within and without Indiana, multiplied by a 
fraction. The numerator of the fraction is the total number of miles traveled in Indiana and the denominator is the sum of miles traveled within and outside of Indiana. If there are no records to show the total number of miles traveled, it is presumed that one gallon is consumed for every four miles traveled. A carrier is entitled to a credit against the tax if the carrier (or lessor operating under the carrier's annual permit) has paid the gasoline or special fuel tax on the motor fuel purchased in Indiana and then consumed the fuel outside of Indiana and paid a gasoline, special fuel, or road tax with respect to the fuel in one or more other states or jurisdictions.

\section{Motor Carrier Surcharge Tax}

This tax is applicable to a carrier who operates or causes operation of a commercial motor vehicle on any highway in Indiana. The tax base is the total amount of motor fuel consumed by commercial motor vehicles operated by a carrier in its operations on highways in Indiana. The tax imposed does not apply to that portion of motor fuel used to propel equipment mounted on a motor vehicle having a common reservoir for locomotion on the highway and the operation of such equipment. The rate of tax is 11 cents per gallon. This tax is in addition to any other motor fuel tax imposed. The amount of motor fuel consumed by a carrier is the total amount of motor fuel consumed in its entire operations within and without Indiana, multiplied by a fraction. The numerator of the fraction is the total number of miles traveled in Indiana and the denominator is the total number of miles traveled within and outside of Indiana. If there are no records to show the total number of miles traveled, it is presumed that one gallon is consumed for every four miles traveled. Tax exemptions include motor vehicles operated by the state, a subdivision of the state, the United States, or an agency of states; a school bus operated on behalf of a state or political subdivision of a state; a vehicle used in casual or charter bus operations; vehicles registered as farm vehicles by the Bureau of Motor Vehicles or under a similar law of another state; trucks with dealer registration plates; and an intercity bus as defined in IC 9-13-2-83. The tax revenue is administered by the Special Tax Division of the Department of Revenue.

\section{Motor Carrier Regulation Fund}

The Motor Carrier Regulation Fund (MCRF) is administered by the Special Tax Division of the Department of Revenue. This fund supports the costs associated with motor carrier officers and weigh station staff. Motor carriers who transport passengers or property for compensation must have a certificate or permit. Annual vehicle registration fees for motor carriers range between "no charge" to a $\$ 10$ maximum, depending upon the state in which the vehicle has a base plate. Registration of interstate operating authority requires $\$ 25$ for the first registration and additional registrations are $\$ 10$ each. Registration fees are waived if the vehicle is from any states that has a reciprocity agreement with the state of Indiana. All revenue collected under Indiana Code 8-2.1 
(Motor Carrier Fees) is deposited in the Motor Carrier Regulation Fund. Any funds remaining in excess of $\$ 500,000$ of the amount appropriated is paid to the MVHA as a reimbursement. However, for the past three years, this criterion has not been met and no reimbursements have been made to the MVHA (Jackson, 2004a).

\section{Motor Fuel Inventory Tax}

Persons having title to gasoline and authorized unlicensed dealers who have special fuel in their taxable storage facilities held for resale on the effective date of an increase in the gasoline or special fuel tax rates are subject to the inventory tax. The tax base comes from the gallons of gasoline or special fuel held for resale and the tax rate is equal to the difference of the increased license tax rate minus the previous license tax rate. This tax revenue is administered by the Special Tax Division of the Department of Revenue and is distributed in the same manner as the gasoline tax and special fuel tax. However, the inventory tax is imposed only when there is an increase in the gasoline tax and or the special fuel tax. Because there was no increase in tax between FY 1991 and FY 2001, there was no revenue collected.

\section{Special Fuel Tax}

The special fuel tax is paid by licensed special fuel suppliers who sell special fuel from a terminal rack located in Indiana, licensed permissive suppliers who sell special fuel for import into Indiana, or persons who purchase special fuel that is tax-exempt and subsequently use the fuel in a taxable manner. The tax base is from special fuel sold or used in producing or generating power for propelling motor vehicles, except fuel used for non-highway purposes, such as for heating oil or in trains. The tax rate is 16 cents per gallon. For FY 2003, the total revenue from the special fuel tax was 218.8 million dollars. The first 25 million is distributed $60 \%$ to local units and $40 \%$ to INDOT. The remainder is deposited $75 \%$ to the MVHA and $25 \%$ to the HRSF as illustrated in Figure 3.5. The tax is administered by the Special Tax Division of the Indiana Department of Revenue.

\subsubsection{Special Distribution Account}

The Special Distribution Account consists of the first 25 million dollars from gas taxes and the first 25 million from special fuel taxes as illustrated in Figure 3.5. Forty percent is distributed to INDOT; 30\% to cities, towns, and counties based on local road and street formulas; and 30\% to cities, towns, and counties based on the Motor Vehicle Highway formula. 


\subsubsection{Debt Service Accounts}

Two accounts in the state highway funding charts were developed for the purpose of covering the lease payments to the Indiana Transportation Finance Authority (ITFA). These accounts are the State Highway and Road Construction and Improvement Fund (SHRCIF) and Crossroads 2000. When these two accounts were created, they were designed to generate enough revenue to cover the amount of bonds being issued. The repayment schedule on the highway leases is staggered over a period of time so that the same payment amount is due each year. After a bond is paid off, its payment amounts are applied to other bond issues. In the past, some residual funds from these two accounts have gone to the SHF. However, after FY 2006, both of these accounts will be 100 percent committed to the repayment of lease payments (Jackson 2004). For the horizon of the present study (2006-2020), all funds in SHRCIF and Crossroads are considered to be committed to the repayment of issued highway infrastructure bonds and are therefore not factored into future highway infrastructure revenue projections.

\section{State Highway and Road Construction and Improvement Fund}

The Indiana legislature created the State Highway and Road Construction and Improvement Fund (SHRCIF) in 1988 by allocating that the first one cent of the gasoline tax paid for each gallon would go exclusively to SHRCIF. It is anticipated that new bonds issued in 2004 will exhaust the revenues from both Crossroads and SHRCIF (Jackson 2004).

\section{The Crossroads 2000 Program}

The Crossroads program was funded by combining a one-time 70 million-dollar allocation from the state's budget surplus, with slight increases in Bureau of Motor Vehicles fees in 1998. The revenue generated from the fee increase was then used to secure bonds to build the projects. More than 100 projects were part of the original Crossroads 2000 bonding program (INDOT 2004a). The money earned through federal and state gasoline taxes, the traditional sources of highway funding, was used for the preservation portion of the Crossroads 2000 program (INDOT 2004a).

The legislators built in a $\$ 200$ million distribution of cash to local municipalities for their road improvement projects. In 2003, 420 million dollars in bonds was issued for projects in 20 different counties constructed between 2002 and 2004. An additional 310 to 350 million dollars in bonds will be issued for projects through the end of 2005 .

\subsubsection{International Registration Program (IRP)}

Under IRP carriers pay registration fees through their base jurisdiction (home state) to jurisdictions in which they travel according to the percent of fleet miles traveled and the fee schedule 
operative in each jurisdiction. The revenue is administered by the Indiana Department of Revenue following the "one-stop shopping" provisions in Indiana Code 6-8.1-4-4. This revenue is distributed to the MVHA.

\subsection{Revenue Projections from Historical Trends for State Highway Agency}

The two largest funds that support state highway network are the State Highway Fund and Federal Aid funds. Table 3.8 presents the historical revenues obtained for state infrastructure work between 1995 and 1998 and Table 3.9 shows the historical revenues obtained between 1999 and 2003. As discussed previously, the Crossroads 2000 fund was established in 1998; therefore, there were no revenues for this fund prior to 1998. The Highway Federal Aid Project Specific resources refer to projects earmarked by the United States Congress.

Table 3.8 State Highway Revenue History 1995 - 1998 (Millions, Current\$)

\begin{tabular}{lcccc}
\hline \multirow{2}{*}{ Resource } & \multicolumn{4}{c}{ Year } \\
\cline { 2 - 5 } & 1995 & 1996 & 1997 & 1998 \\
\hline State Highway Fund & 525.2 & 549.3 & 517.9 & 522.6 \\
General Fund & - & - & - & 35.0 \\
SHRCIF & 53.6 & 50.6 & 39.9 & 36.1 \\
Bond Program & 2.0 & - & - & 168.0 \\
Crossroads 2000 & - & - & - & 17.2 \\
Hwy. Fed. Aid Regular & 239.3 & 288.0 & 323.0 & 302.5 \\
Hwy. Fed. Aid Project Specific & 4.8 & 32.0 & 19.0 & 20.8 \\
\hline Total Resources & 825 & 920 & 900 & 1,102 \\
\hline Jackson (2004b) & & &
\end{tabular}

Table 3.9 State Highway Revenue History 1999 - 2003 (Millions, Current\$)

\begin{tabular}{lccccc}
\hline \multirow{2}{*}{ Resource } & \multicolumn{5}{c}{ Year } \\
\cline { 2 - 6 } & 1999 & 2000 & 2001 & 2002 & 2003 \\
\hline State Highway Fund & 584.9 & 587.9 & 566.7 & 555.8 & 612.7 \\
General Fund & 35 & - & - & $-30)$ & - \\
SHRCIF & 49.3 & 50.7 & 51.0 & 51.7 & 59.4 \\
Bond Program & - & 102.2 & 129.8 & 133.5 & 249.0 \\
Crossroads 2000 & 52.4 & 35.3 & 51.2 & 47.0 & 60.9 \\
Hwy. Fed. Aid Regular & 428.2 & 412.9 & 465.3 & 461.1 & 430.1 \\
Hwy. Fed. Aid Project Specific & 28.6 & 4.5 & 26.8 & 20.0 & 23.0 \\
\hline Total Resources & 1,178 & 1,194 & 1,291 & 1,239 & 1,435 \\
\hline
\end{tabular}
Jackson (2004b) 
The three-year annual average level of resources in the State Highway Fund between 2000 and 2002, was 570 million in current dollars. The three-year annual average of total state highway revenues from all sources between 2000 and 2002 was 1.24 billion in current dollars. The three-year annual average of regular federal aid was 446.4 million for the same period. Figure 3.6 presents historical trends in state highway revenue by major category.

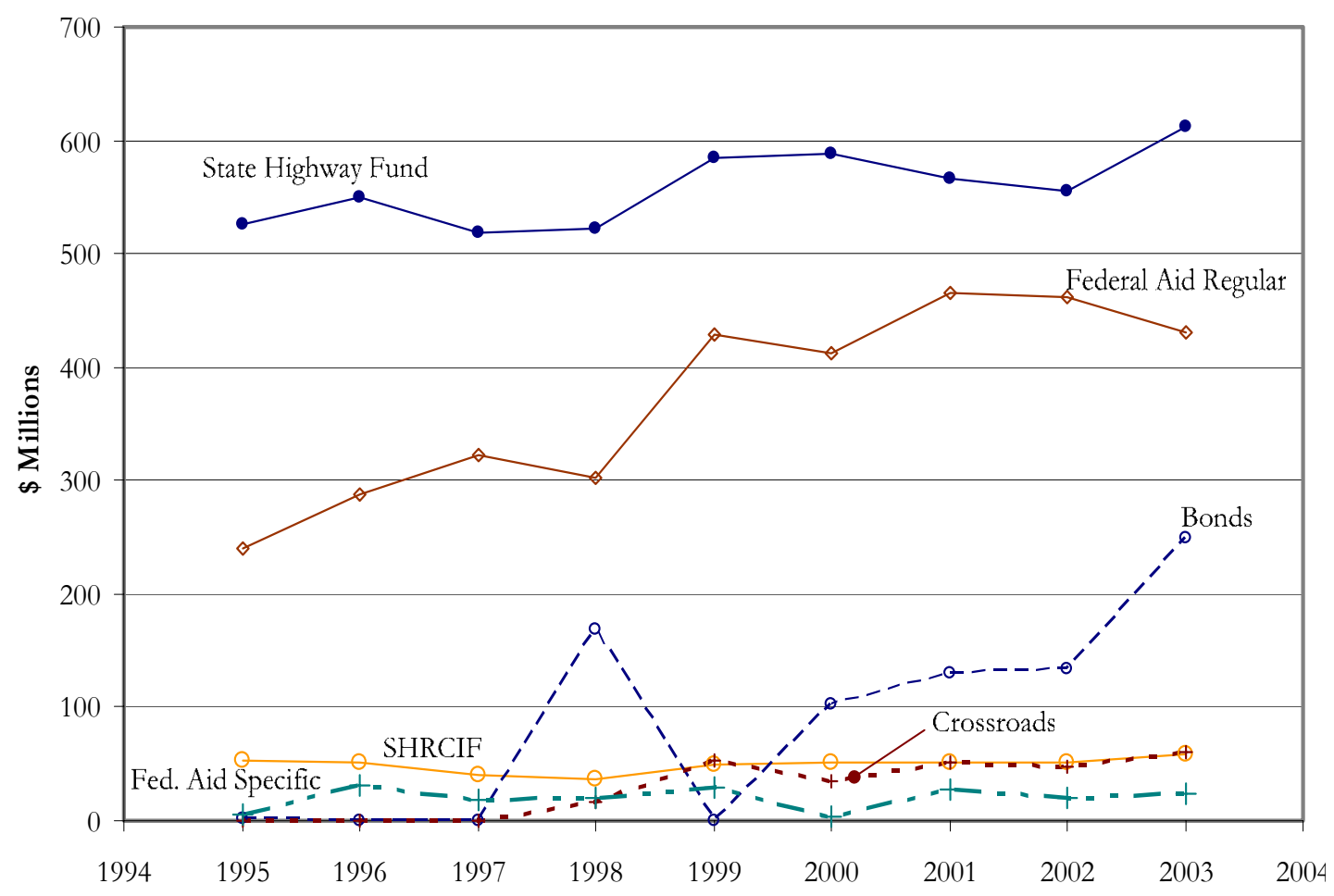

Figure 3.6 Historical Trends in Highway Revenue (Jackson 2004b)

Between 1995 and 2002, the total resources available from all sources for INDOT totaled 8.6 billion in current dollars. To investigate the impact of inflation on the total resources obtained over this eight- year period, the 1995 constant dollar equivalent was calculated for each year. The current dollar amount for each year was compared to its equivalent 1995 constant dollar amount and any differences were noted. Over the period between 1995 and 2002, it was determined that there was a cumulative loss in state highway revenue due to inflation in the amount of 832.7 million in constant 1995 dollars, as shown in Table 3.10. 
Table 3.10 Inflation Impact of Highway Revenue (Millions)

\begin{tabular}{crrr}
\hline Year & $\begin{array}{r}\text { Total } \\
\text { Resources } \\
\text { Current\$ }\end{array}$ & $\begin{array}{r}\text { Total } \\
\text { Resources } \\
1995 \$\end{array}$ & Difference \\
\hline 1995 & 824.9 & 824.9 & - \\
1996 & 919.9 & 932.9 & 13.0 \\
1997 & 899.8 & 839.8 & $(59.9)$ \\
1998 & $1,102.2$ & $1,058.8$ & $(43.4)$ \\
1999 & $1,178.4$ & $1,052.4$ & $(126.0)$ \\
2000 & $1,193.5$ & 999.3 & $(194.3)$ \\
2001 & $1,290.8$ & $1,086.7$ & $(204.1)$ \\
2002 & $1,239.1$ & $1,021.3$ & $(217.8)$ \\
\hline Total & $8,648.8$ & $7,816.0$ & $(832.7)$ \\
\hline Jackson (2004b); FHWA (2004). & &
\end{tabular}

On the basis of historical data, the 15-year revenue for year 2006-2020 was estimated using two scenarios each for SHF and Federal Aid revenue. The two scenarios considered for SHF were a) 6-year average held, and b) No revenue increase. The 15-year revenue projected considering 6-year average held for SHF was estimated to be $\$ 8.9$ billion, whereas for the no revenue increase scenario the 15 -year revenue was estimated to be $\$ 7.1$ billion. In the similar manner, two scenarios considered for Federal Aid were a) 6-year average held and b) 3-year average held. The 15-year revenue projected considering 6-year average held for Federal Aid was estimated to be $\$ 6.3$ billion, whereas for the 3-year average held scenario the 15-year revenue was estimated to be $\$ 6.8$ billion. Different combinations of the SHF and Federal Aid revenue gives four possible combinations of the total 15-year projected revenue for the state highway agency, as shown in Table 3.11.

Table 3.11 15-year Projected Revenue for State Highway Agency on the Basis of Historical Trends

\section{Revenue Source}

6-year average held

No revenue increase
Projected Revenue

$\$ 8.9$

$\$ 7.1$

$\$ 6.3$

$\$ 6.8$

15-year Revenue Total

$\$ 15.20$

$\$ 13.40$

$\$ 15.70$

$\$ 13.90$ 


\subsection{Historical Trends in Revenues for Local Agencies}

The two major sources of revenue for local agencies are the Motor Vehicle Highway Account (MVHA) and the Highway Road and Street Fund (HRSF). Forty-seven percent (47\%) of MVHA is distributed to local agencies, whereas $45 \%$ of the amount in HRSF is distributed to the Local Road and Street Fund (LRSF). Besides these two state revenue sources, local governments also get funding through Federal Aid and they have the option to raise funds independently through different local taxes. Figure 3.7 shows the revenue distribution through different funds for the calendar year 2001 for Indiana local agencies.

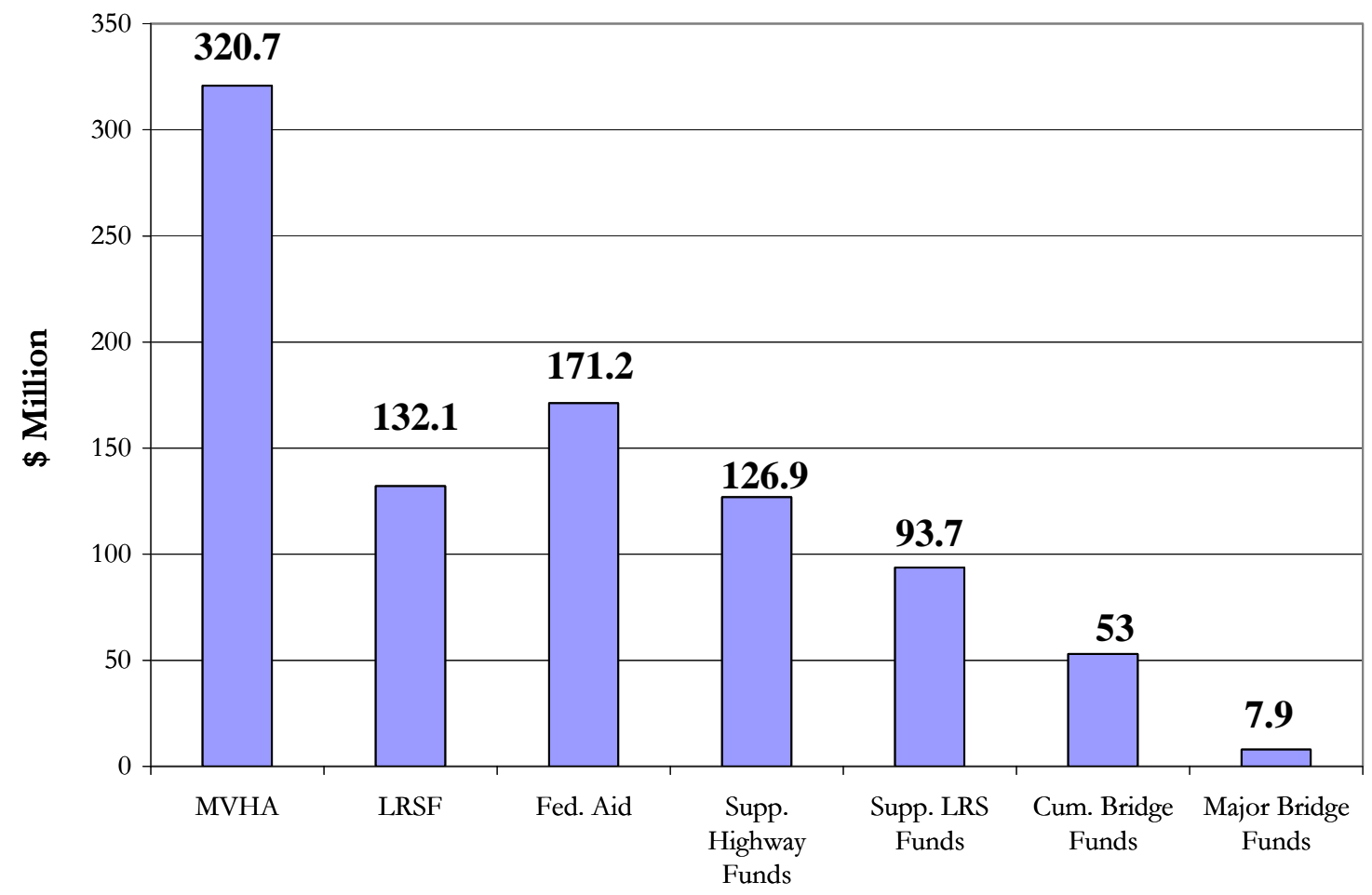

Figure 3.7: Sources of Highway Revenue for Indiana Local Agencies (CY 2001)

Both MVHA and HRSF revenues are derived from motor fuel taxes and registration and license fees. Since January 1, 2003, a one cent increase per gallon on gasoline/gasohol tax has gone directly to local agencies. Figure 3.8 illustrates the Indiana transportation funding for local governments. 


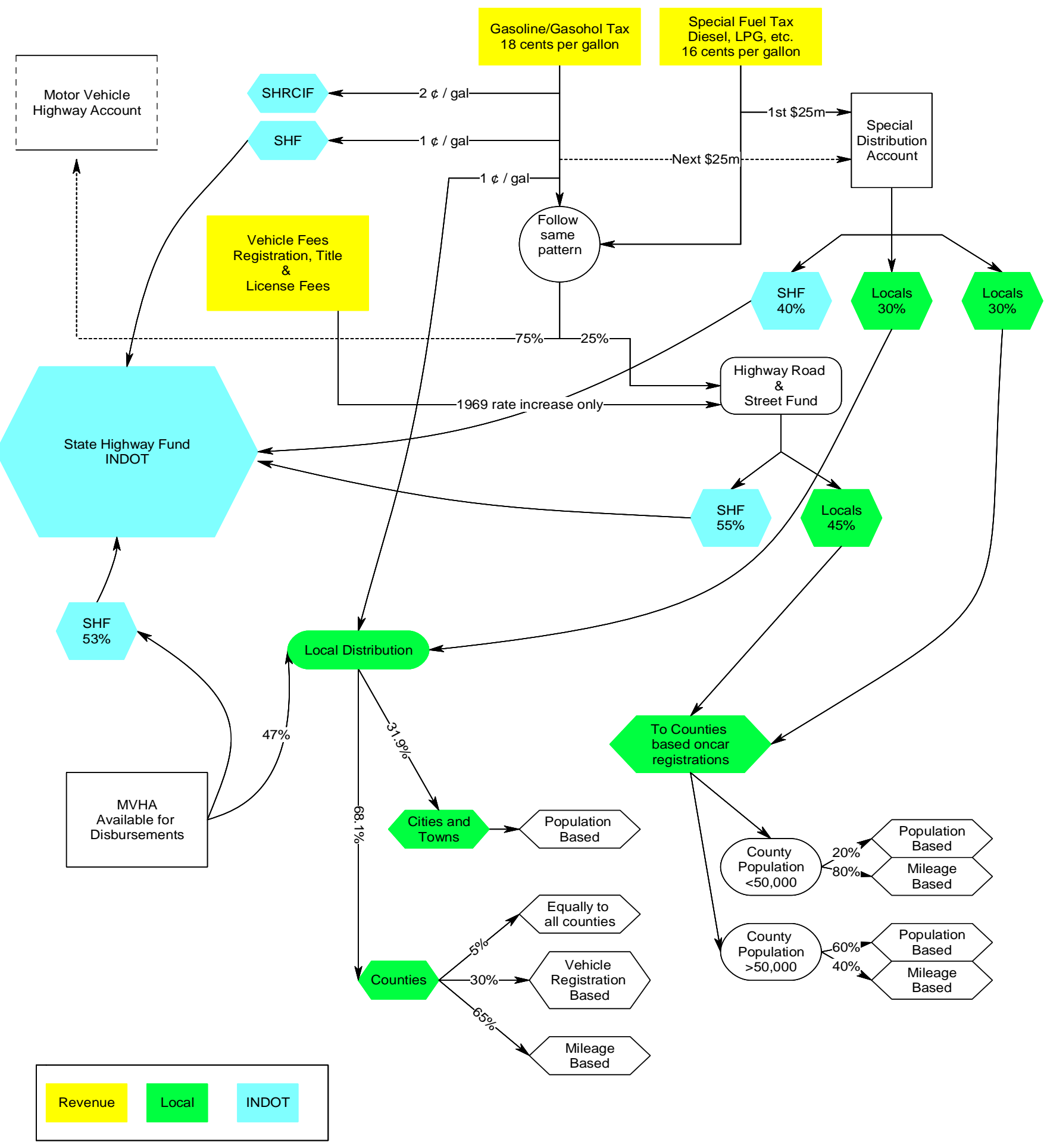

Figure 3.8: Indiana Transportation Funding for Local Governments

Source: INDOT Budget and Fiscal Management Division (2003)

(Federal funds and local option taxes are not included) 
Figure 3.9 shows the historical trends for MVHA and HRSF for a 10-year period (19932002) for state fiscal years. These values represent the revenue available to the entire state before the distribution to INDOT and local agencies. All values are brought to 2002 dollars.

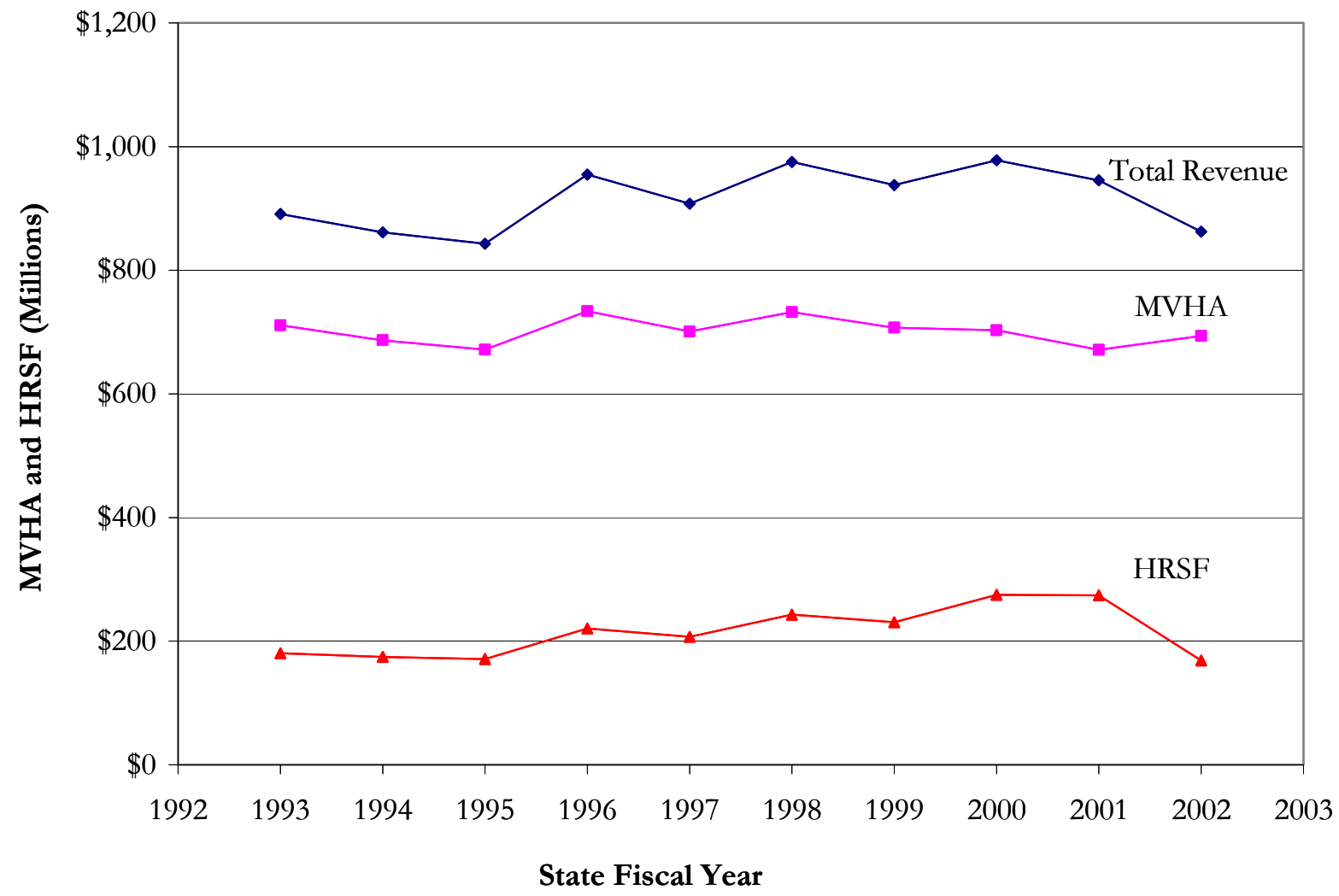

*State fiscal year begins $7 / 1$ and ends $6 / 30$

** All values are expressed in 2002 dollars

Average total revenue $=\$ 920,033,130.21$

Figure 3.9 MVHA and HRSF Revenue Trends for State Fiscal Years 1992-2002

\subsubsection{Motor Vehicle Highway Account (MVHA)}

The MVHA is the primary source of funding for local agencies. MVHA may be used for all legal expenses of the agency, including administrative and operational expenses, road maintenance and construction, equipment maintenance and replacement, snow and ice control, fuel, and other supplies. Figure 3.10 shows the different sources and revenue trends over the fiscal years 1992 to 
2002 for the MVHA before the distribution to INDOT and local agencies. Table 3.12 shows the amounts received by local governments from the MVHA for the fiscal years 1990-2000.

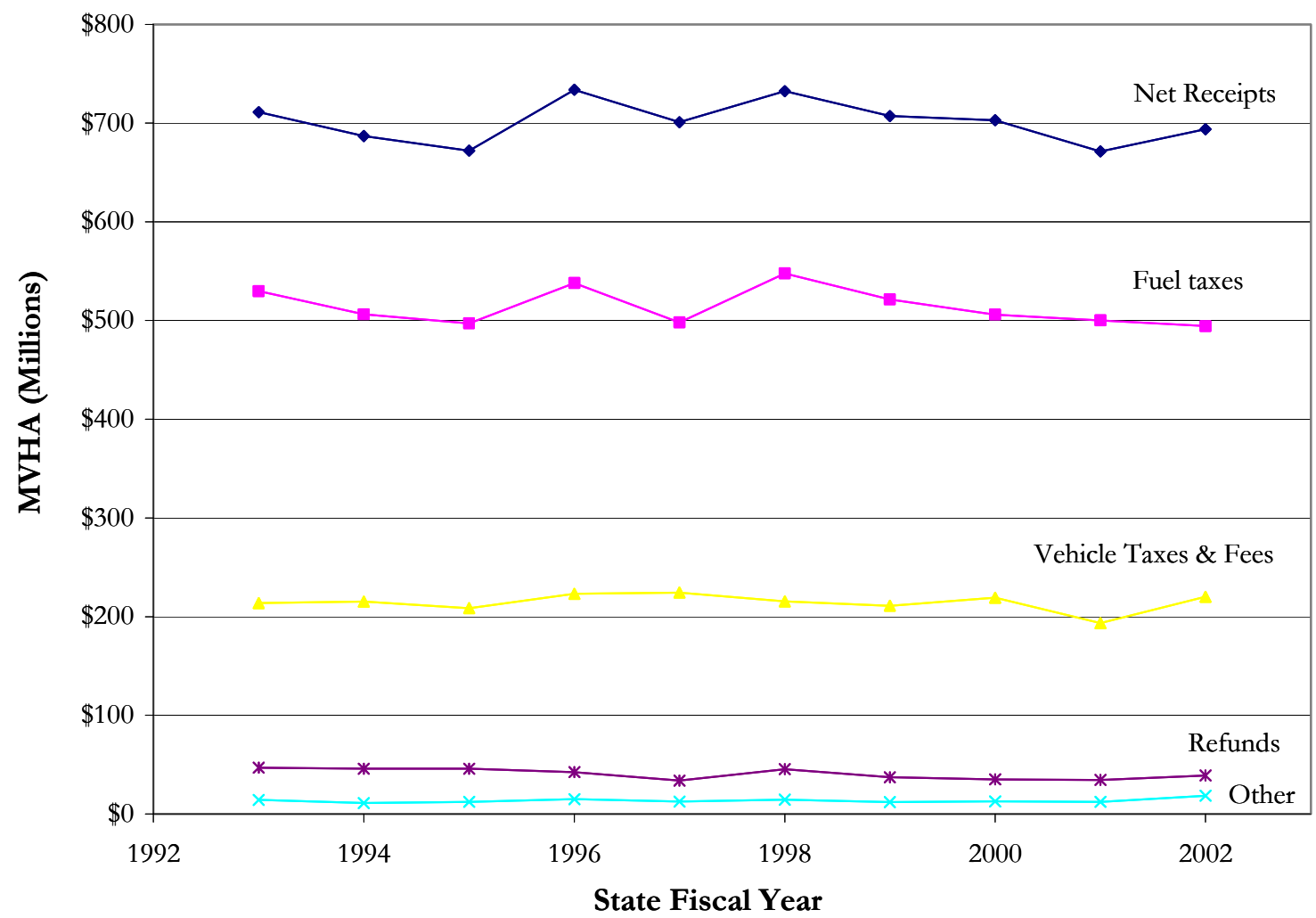

*State fiscal year begins $7 / 1$ and ends $6 / 30$

** All values are expressed in 2002 dollars

Average revenue from MVHA $=\$ 701,042,878.42$

Figure 3.10: MVHA Revenue Sources for State Fiscal Years 1992-2002 
Table 3.12 MVHA Revenue for Indiana Local Agencies (1990-2000 FY)

\begin{tabular}{|c|c|c|c|}
\hline State Fiscal Year & Counties & Cities and Towns & Total \\
\hline $99-00$ & $\$ 204,626,891$ & $\$ 98,908,010$ & $\$ 303,534,901$ \\
\hline $98-99$ & $\$ 208,758,034$ & $\$ 101,063,337$ & $\$ 309,821,371$ \\
\hline $97-98$ & $\$ 214,396,840$ & $\$ 103,944,689$ & $\$ 318,341,529$ \\
\hline $96-97$ & $\$ 211,129,607$ & $\$ 102,240,178$ & $\$ 313,369,785$ \\
\hline $95-96$ & $\$ 221,689,985$ & $\$ 107,427,375$ & $\$ 329,117,360$ \\
\hline $94-95$ & $\$ 198,427,715$ & $\$ 96,459,579$ & $\$ 294,887,294$ \\
\hline $93-94$ & $\$ 217,038,830$ & $\$ 105,368,707$ & $\$ 322,407,536$ \\
\hline $92-93$ & $\$ 219,125,156$ & $\$ 106,757,876$ & $\$ 325,883,032$ \\
\hline $91-92$ & $\$ 215,757,432$ & $\$ 105,204,380$ & $\$ 320,961,812$ \\
\hline $90-91$ & $\$ 208,735,334$ & $\$ 101,838,089$ & $\$ 310,573,423$ \\
\hline
\end{tabular}

\subsubsection{Local Road and Street Fund (LRSF)}

The money allocated from HRSF to local agencies goes into the Local Road and Street Fund (LRSF), which may be used only for specific types of expenses permitted by IC 8-14-2. Most agencies dedicate the LRSF distribution entirely to the maintenance of their roads and streets, although there are several other legal uses, including purchases of equipment, obtaining right of way, and engineering services. The LRSF distribution has been increased by $\$ 25$ to $\$ 100$ million per year from 1995 to 2001 by a transfer from the state general fund. The revenue available to local agencies in the LRSF through the HRSF for the fiscal years 1990-1991 to 1999-2000 is documented in Table 3.13. 
Table 3.13: HRSF Revenue for Indiana Local Agencies (1990-2000 FY)

\begin{tabular}{|c|c|c|c|}
\hline State Fiscal Year & Counties & Cities and Towns & Total \\
\hline $99-00$ & $\$ 101,944,208$ & $\$ 77,731,156$ & $\$ 179,675,364$ \\
\hline $98-99$ & $\$ 75,818,920$ & $\$ 57,811,860$ & $\$ 133,630,779$ \\
\hline $97-98$ & $\$ 80,223,156$ & $\$ 61,193,475$ & $\$ 141,416,630$ \\
\hline $96-97$ & $\$ 63,630,349$ & $\$ 48,186,268$ & $\$ 111,816,617$ \\
\hline $95-96$ & $\$ 68,384,374$ & $\$ 51,252,389$ & $\$ 119,636,763$ \\
\hline $94-95$ & $\$ 44,021,945$ & $\$ 32,993,354$ & $\$ 77,015,298$ \\
\hline $93-94$ & $\$ 44,766,048$ & $\$ 33,770,878$ & $\$ 78,536,926$ \\
\hline $92-93$ & $\$ 46,259,991$ & $\$ 34,897,889$ & $\$ 81,157,880$ \\
\hline $91-92$ & $\$ 46,278,891$ & $\$ 34,912,145$ & $\$ 81,191,036$ \\
\hline $90-91$ & $\$ 44,020,479$ & $\$ 33,740,479$ & $\$ 77,760,958$ \\
\hline
\end{tabular}

\subsubsection{Local Supplemental Funding}

Supplemental funds can be raised by counties using different taxes and user fees such as the County Option Income Tax, County Option Vehicle Tax, Gaming Funds, miscellaneous taxes, etc. While many counties can find ways to rise supplemental funding, the money received can vary widely. Populated counties can receive more money from local income and vehicles taxes than the less populated and rural counties (LTAP 2001). At present, the majority of the counties have low levels of supplemental funding. In 2001, Hamilton County had the highest level of supplemental highway funding with nearly $\$ 16$ million, whereas Randolph had no supplemental highway funding. Total supplemental funding available to all local agencies in CY 2001 was $\$ 220,589,277$.

The following taxes are included in each of the tax categories used for supplemental funding:

\section{County Option Income Taxes:}

- CEDIT (County Economic Development Income Tax):25 counties

- CAGIT (County Adjusted Gross Income Tax): 8 counties

- COIT (County Option Income Tax): 5 counties

\section{County Option Vehicle Taxes}

- LOHUT (Local Option Highway User Tax) : 24 counties

- Non-motorized Vehicle tax : 10 counties

\section{Permits and User fees}


- Franchise Fund : money received from the Cable Company to repair any road or ROW damage (2 counties)

- Permits: road cuts, underground and driveway permits and ROW permits (28 counties)

- Parking: Money generated from the use of publicly owned parking meters and parking garages ( 2 counties)

\section{Gaming Funds}

- Boat money: Portion of River Boat gambling revenues (10 counties)

- Build Indiana Funds: Portion of Lottery tickets (4 counties), Application process involved in obtaining this money

\section{Miscellaneous County Taxes}

- County General Fund: Certain counties are attributed a certain amount from the County General Fund (7 counties)

- Capital Development Fund: Levy on property to raise money for capital improvements within the county ( 9 counties)

- Tax Increment Financing: Increased assessed value of land due to industrial development (4 counties)

\section{Refunds/Reimbursements}

- Engineer Salary Subsidy: maximum of $\$ 20,000$ from the State of Indiana to help subsidize the salary of the county engineer (48 counties)

- Covered Bridge Subsidy: Subsidy counties receive for the maintenance and repair of covered bridges within their jurisdiction (29 counties)

- Fuel Reimbursement: refund allotted for fuel tax paid (23 counties)

- Disaster Relief: Money available to repair infrastructure damaged by natural disasters(29 counties)

Miscellaneous: Money generated by the counties for their highway departments (Sale of Salvage, Solid waste, Bonding, Interest on Investments, Cost Sharing Programs, Outsourcing of Equipment/Personnel)

\section{Counties Municipal Option Income Taxes}

- LOHUT (Local Option Highway User Tax)

- EDIT (Economic Development Income Tax)

- Local Option Income Tax 


\section{Miscellaneous Municipal Taxes}

- General Fund

- Cumulative Capital Development Fund

- Thoroughfare Fund

- Cumulative Capital Improvement Fund

Other Sources: Bond Fund, Gaming, Interest on Investment, Sale of Salvage, Community Development Block Grant Fund, Disaster Relief, and Solid Waste Permits.

\subsubsection{Federal Aid}

The Federal government makes a commitment to pay the State for the Federal share of a project's eligible cost. This commitment occurs when the project is approved and the project agreement is executed. Federal Aid grants are given to state and local governments. Figure 3.11 shows a summary of Federal Aid revenues, for federal fiscal years 1997-2002, including Bridge Federal Aid. Table 3.14 shows the Federal Aid Fund categories for local use. 


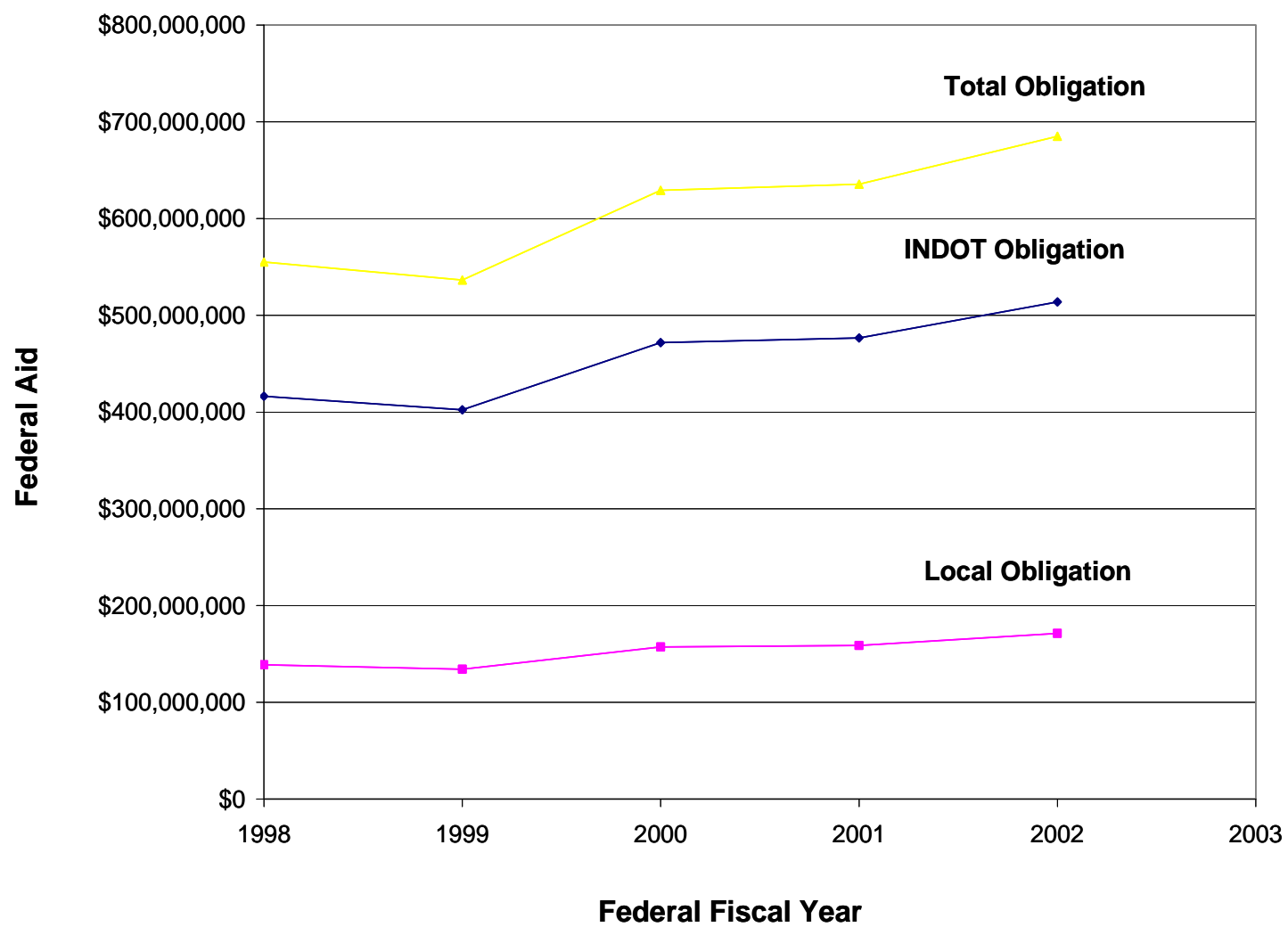

*Federal fiscal year begins $10 / 1$ and ends $9 / 30$

** All values are expressed in 2002 dollars

Figure 3.11: Federal Aid: Federal Fiscal Years 1997-2002

Table 3.14: Summary of Federal Aid Distributions for Local Use By Fund Category (5-Year Federal Fiscal Year Period)

\begin{tabular}{|c|c|c|c|c|c|c|}
\hline $\begin{array}{c}\text { Federal } \\
\text { Fiscal Year }\end{array}$ & Bridge & STP & CMAQ & $\begin{array}{c}\text { Minimum } \\
\text { Guarantee }\end{array}$ & Other & Total \\
\hline $01-02$ & $\$ 19,430,662$ & $\$ 120,625,212$ & $\$ 10.354,559$ & $\$ 20,844,746$ & $\$ 0$ & $\$ 171,255,179$ \\
\hline $00-01$ & $\$ 18,200,501$ & $\$ 108,476,689$ & $\$ 9,253,301$ & $\$ 19,632,590$ & $\$ 0$ & $\$ 155,563,081$ \\
\hline $99-00$ & $\$ 17,882,350$ & $\$ 104,324,236$ & $\$ 8,714,270$ & $\$ 23,932,778$ & $\$ 0$ & $\$ 154,853,634$ \\
\hline $98-99$ & $\$ 13,905,425$ & $\$ 82,958,098$ & $\$ 6,610,406$ & $\$ 20,306,872$ & $\$ 0$ & $\$ 123,780,801$ \\
\hline $97-98$ & $\$ 10,662,472$ & $\$ 86,124,129$ & $\$ 5,379,002$ & $\$ 11,955,915$ & $\$ 4,933,410$ & $\$ 119,054,928$ \\
\hline
\end{tabular}

STP: Surface Transportation Program Funds

CMAQ: Congestion Mitigation/ Air Quality Funds 


\subsubsection{Dedicated Sources of Revenue for Local Bridges}

Funds to maintain and replace different bridges come mainly from Cumulative Bridge Funds, Major Bridge Funds and Federal Aid.

\subsubsection{Cumulative Bridge Funds}

Cumulative Bridge Funds are the major funding source for Indiana counties to maintain and replace bridges. In 2001, 89 of 92 counties utilized the Cumulative Bridge Fund as their primary source of funds for bridge repair and replacement. The cumulative bridge fund is a tax based on property, with a statutory maximum of $\$ 0.30$ per $\$ 100.00$ assessed valuation. As the generated money is proportionate to the net assessed value of the county, cumulative bridge fund is not as effective in large rural counties as in smaller and more developed counties. Due to this dependence on the size and the degree of development of the counties, funding in 2000 on a per bridge basis went as low as $\$ 685$ per year per bridge in Rush County to as high as $\$ 19,553$ per year per bridge in Lake County (LTAP 2001). In 2001, Indiana counties received $\$ 53$ million coming from Cumulative Bridge Fund.

\subsubsection{Major Bridge Funds}

In Indiana, only five counties have been allowed to enact legislation establishing a major bridge fund. Indiana code contains very specific requirements on which counties may utilize this fund. Population criteria determine which counties are eligible, and size and use criteria determine what constitutes a major bridge. Based on these criteria and a review of the existing bridge inventory data, it is estimated that less than 100 bridges (less than $1 \%$ of the statewide total) qualify for major bridge funding in the five enacting counties (LTAP 2001). Table 3.15 shows the counties that used cumulative bridge funds in 2001 . 
Table 3.15: 2001 (CY) County Major Bridge Fund Revenues

\begin{tabular}{|c|c|}
\hline County & Amount \\
\hline Allen & $\$ 1,328,001$ \\
\hline Elkhart & $\$ 1,923,092$ \\
\hline Hamilton & $\$ 2,420,840$ \\
\hline LaPorte & $\$ 288,223$ \\
\hline St. Joseph & $\$ 1,928,245$ \\
\hline Total & $\$ 7,888,401$ \\
\hline
\end{tabular}

\subsubsection{Federal Aid for Bridges}

Traditionally, the state and the counties share Federal bridge aid funds on the basis of a $65 / 35 \%$ split. A minimum of $15 \%$ and a maximum of $35 \%$ of the federal aid bridge funds are required to be spent on "off system" bridges. However, all "off systems" bridges are located in the county system, but not all county bridges are "off system". Hence, the $35 \%$ limit is applicable to "off system" bridges and not to county bridges in general. For federal fiscal year 2001, Federal Bridge Aid funds attained nearly $\$ 21$ million due to a transfer of money from another category (LTAP 2001).

\subsubsection{Revenue Projections for Local Agencies}

The task of projecting the revenue over the period of study is critical because one part of the revenue which is based on fuel taxes and registration fees is relatively stable and the other part which basically constitutes non fuel tax revenue is highly variable. Furthermore, information on supplemental revenue and revenue from bridge dedicated funds for Indiana local agencies is available for only the year 2001. Therefore, the approach used to predict future revenue is to use projection methods for MVHA, LRSF and Federal Aid on the basis of available data.

\subsubsection{Local Revenue Projections Based on MVHA and LRSF}

Historical data was compiled and analyzed for each of these funds. The revenue available through MVHA showed high variability in data and hence projection based on the historical trends 
does not give a good model. For the LRSF, an increasing trend was observed. However, projections based on these values would lead to high LRSF values for the future years due to high increase observed in the last 5 years. This type of projection would be misleading because of the bias caused by the recent increase which may be only temporal. However, if the revenues from the two funds are combined and analyzed for the 10 years of data available, a reasonable trend can be established. The three models with the highest $\mathrm{R}^{2}$ values were considered: the linear form, the logarithmic form and the exponential form. The linear and logarithmic forms were used to establish a range of predicted revenue over the study period. The results obtained and the models developed are shown in Figures 3.12 and 3.13 .

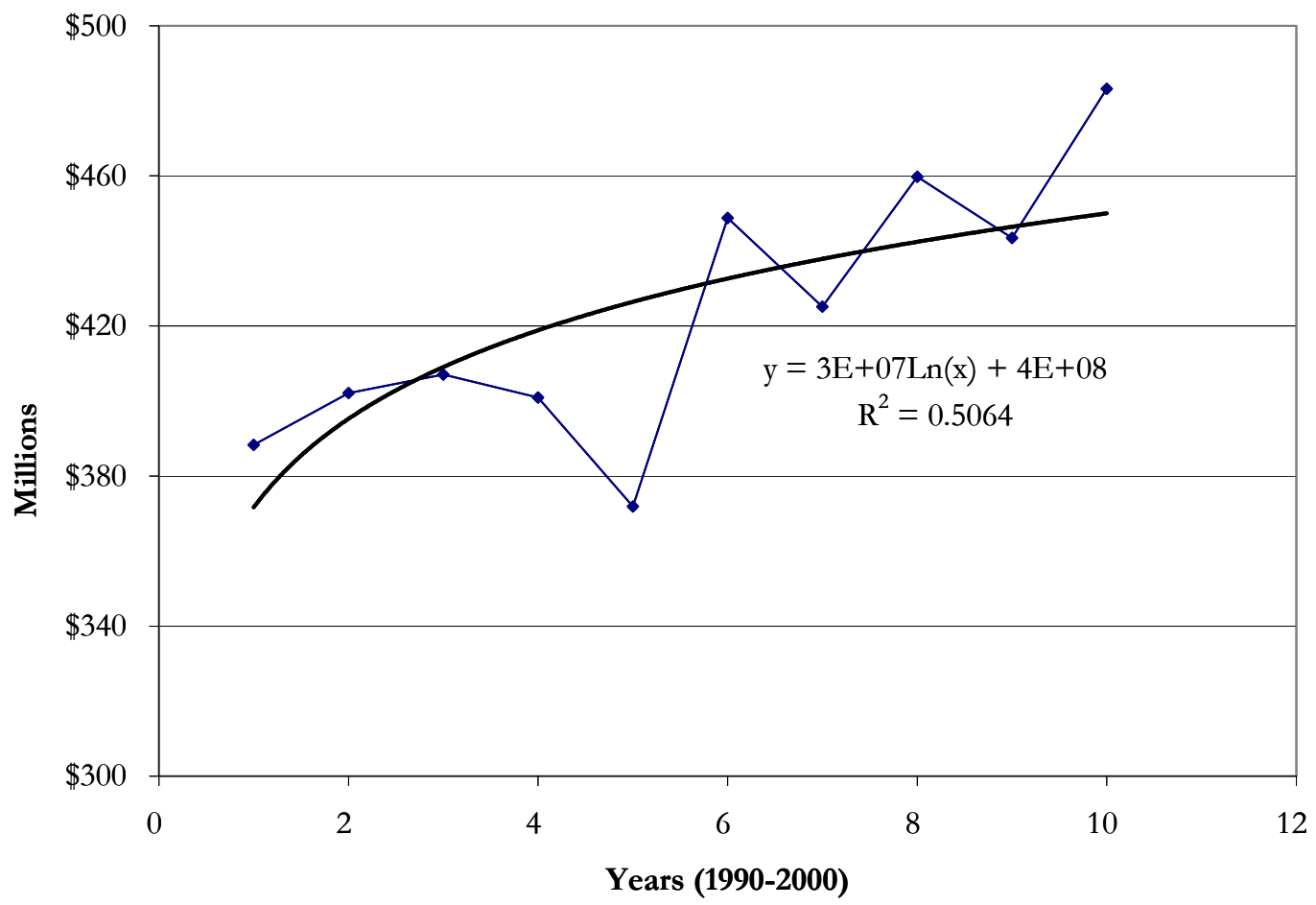

Figure 3.12 MVHA and LRSF Combined Revenue for Indiana Local Agencies (Logarithmic Form) 


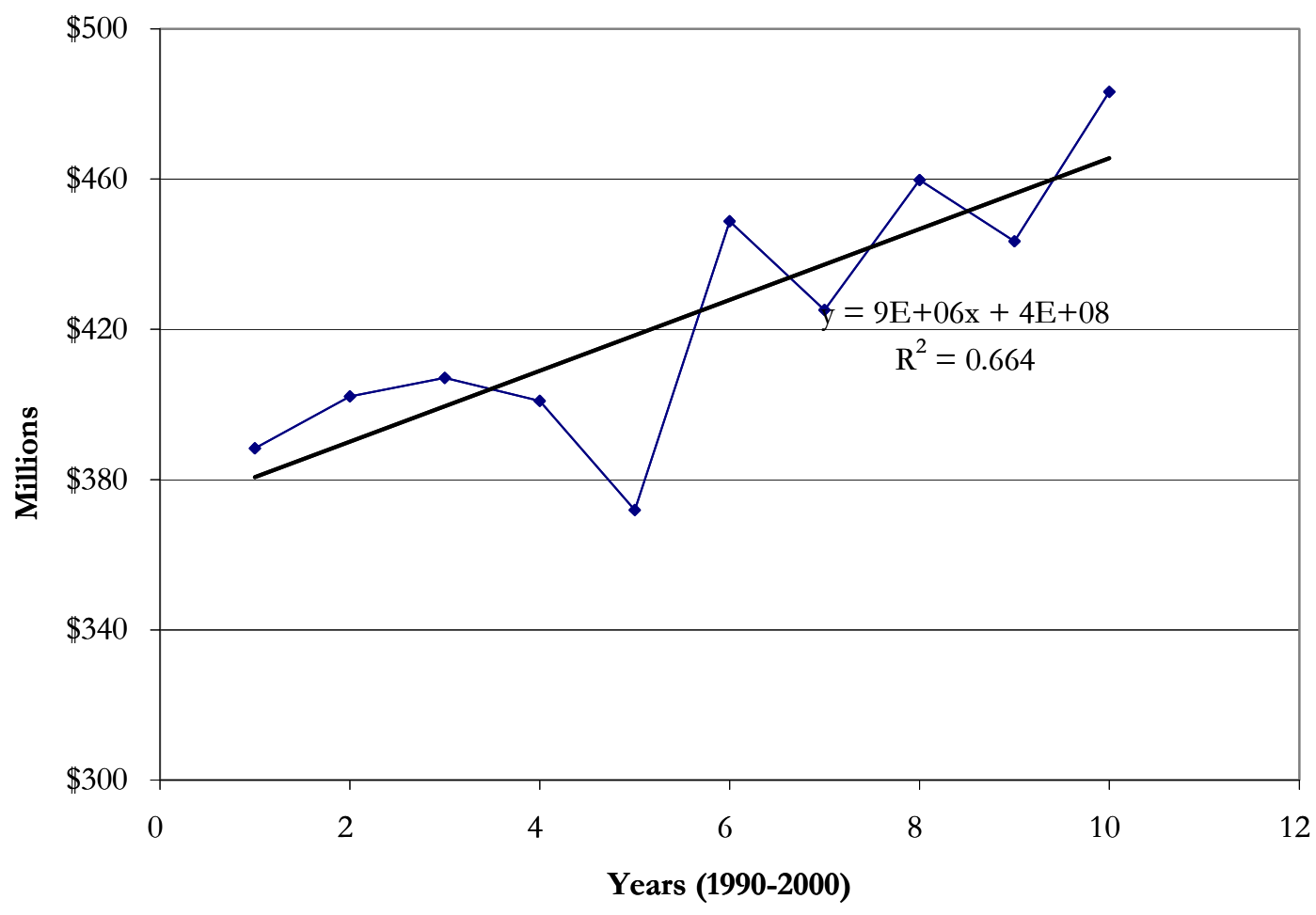

Figure 3.13 MVHA and LRSF Combined Revenue for Indiana Local Agencies (Linear Form)

Although the exponential form had the highest value of $\mathrm{R}^{2}$, the possibility of an exponential growth of fuel tax and registration revenue was discarded. Using the linear and the logarithmic forms, MVHA and LRSF combined revenue can be predicted for the study period using the following equations respectively:

$$
\begin{gathered}
M V H A \text { and } L R S F \text { Revenue }=3 E+07^{*} L n(\# \text { of years from 1990) }+4 \mathrm{E}+08 \\
M V H A \text { and } L R S F \text { Revenue }=9 E+06^{*}(\# \text { of years from 1990 })+4 E+08
\end{gathered}
$$

Table 3.16 shows the expected range of local revenue from the MVHA and LRSF for the study period (2006-2020) using the linear and logarithmic models. 
Table 3.16 MVHA and LRSF Revenue Projection for Local Agencies

\begin{tabular}{|c|c|c|}
\hline \multirow{2}{*}{ Year } & \multicolumn{2}{|c|}{ Projected Revenue (MVHA and LRSF) } \\
\cline { 2 - 3 } & Logarithmic form & Linear form \\
\hline 2006 & $\$ 483,177,662$ & $\$ 544,000,000$ \\
\hline 2007 & $\$ 484,996,400$ & $\$ 553,000,000$ \\
\hline 2008 & $\$ 486,711,153$ & $\$ 562,000,000$ \\
\hline 2009 & $\$ 488,333,169$ & $\$ 571,000,000$ \\
\hline 2010 & $\$ 489,871,968$ & $\$ 580,000,000$ \\
\hline 2011 & $\$ 491,335,673$ & $\$ 589,000,000$ \\
\hline 2012 & $\$ 492,731,274$ & $\$ 598,000,000$ \\
\hline 2013 & $\$ 494,064,826$ & $\$ 607,000,000$ \\
\hline 2014 & $\$ 495,341,615$ & $\$ 616,000,000$ \\
\hline 2015 & $\$ 496,566,275$ & $\$ 625,000,000$ \\
\hline 2016 & $\$ 497,742,896$ & $\$ 634,000,000$ \\
\hline 2017 & $\$ 498,875,106$ & $\$ 643,000,000$ \\
\hline 2018 & $\$ 499,966,135$ & $\$ 652,000,000$ \\
\hline 2019 & $\$ 501,018,875$ & $\$ 661,000,000$ \\
\hline 2020 & $\$ 502,035,921$ & $\$ 670,000,000$ \\
\hline TOTAL $=\$ 7,402,768,948$ to $\$ 9,105,000,000$ \\
\hline
\end{tabular}

\subsubsection{Projection of Federal Aid Revenue}

For the revenue available through Federal Aid, five years of data was available; a trend was thus established as shown in Figure 3.14. The following developed equation allows us to predict Federal Aid for the study period:

Federal Aid Revenue $=2 E+07^{*} \operatorname{Ln}(\#$ of years from 1997) $+1 E+08$

For the study period (2006-2020), $\$ 2.2$ billion can be expected from Federal Aid Revenue for Indiana local agencies. 


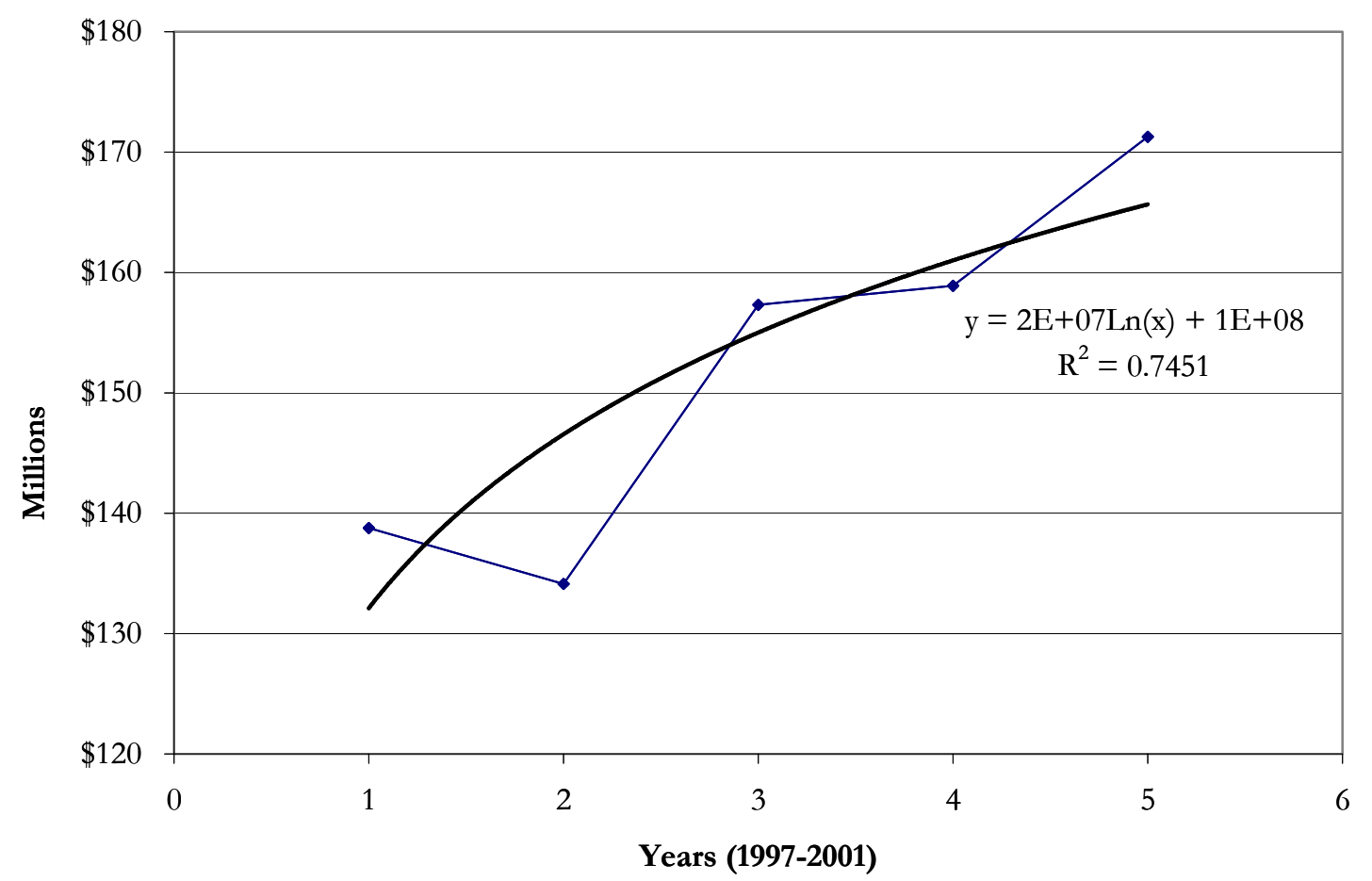

Figure 3.14: Federal Aid Revenue Projection for Indiana Local Agencies

The data for supplemental revenue and bridge dedicated revenue is available for only one year, as shown in Table 3.17. Assuming this yearly amount is available over the analysis period, a total of $\$ 4.2$ billion is expected from these sources.

Table 3.17: 2001 Supplemental Revenue for Indiana Local Agencies

\begin{tabular}{|c|c|}
\hline Revenue Source & Receipt in 2001 (Year 2002 \$) \\
\hline Supp. Hwy Funds & $\$ 129.6$ Million \\
\hline Supp. LRS Funds & $\$ 95.7$ Million \\
\hline Cum. Bridge Funds & $\$ 54.1$ Million \\
\hline Major Bridge Funds & $\$ 8.07$ Million \\
\hline Total & $\$ 287.5$ Million \\
\hline
\end{tabular}




\subsection{Highway Revenue Forecasting}

Apart from the simple projections of revenue forecasting from the historical trends for the state and local agencies as discussed in the previous sections, future highway revenues were also estimated using a detailed forecasting methodology. In the following paragraphs the highway revenue forecasting methodology is discussed. For the purpose of strategic financing planning in Indiana a tool was developed by Varma et al. (1991) that can estimate future revenues under various socioeconomic and policy scenarios. This forecasting model was updated to reflect changes in travel pattern and fuel efficiency as well as changes in registration and fuel tax structures during the past decade.

\subsubsection{Highway Revenue Forecasting Methodology}

The software INDOTREV as well as the detailed highway revenue forecasting methodology developed by Varma et al. (1991) was revised to include recalibrated vehicle fuel efficiency and economic parameters. In the last couple of decades there has been volatility associated with fuel supply and pricing. Also, changes in vehicular technology have resulted in improved fuel economy. A cross sectional approach, as opposed to a time series approach, was used for preparing the forecasting models which included several socio-economic parameters. The software can provide revenue projections under various taxation policies.

\subsubsection{Highway Revenue Forecasting Models}

Highway revenues are largely dependent on travel demand. The travel demand is made up of needs for either personal mobility or movement of goods and commodities. Two major parameters that reflect travel demand are vehicle ownership and vehicle use. Vehicle ownership can be indicated by vehicle registrations. Vehicle use, both for automobiles and commercial vehicles, can be related to economic climate. The methodology disaggregates total revenue into seven major categories: registration, driver license, international registration plan, gasoline tax, special fuel tax, motor carrier surtax and motor carrier fuel use tax. The revenue for each of these categories is separated into specific tax rate and revenue base. Four elements which have been considered in estimating the revenue bases are: 1) population forecasts 2) economic forecasts (gross state product, per capita income) 3) estimation of vehicle-miles of travel (VMT), and 4) fleet fuel efficiency projections. 
The overall procedure for the state generated highway revenue forecasting methodology is given in Figure 3.15. For the registration revenue estimation, the vehicles are divided into seven different categories: automobiles, motorcycles, light duty trucks, tractors, buses, trailers and semitrailers. Light duty trucks, tractors, trailers, and semitrailers are further divided into farm and non-farm categories. Figures 3.16 and 3.17 represent the overall structure of how vehicle registration revenues are estimated.

For fuel tax revenue, vehicle miles of travel by various vehicle categories and associated fleet fuel efficiencies were projected. A cohort survival method was employed for this purpose. Personal vehicle travel with automobiles and a significant portion of light duty trucks is estimated based on economic parameters affecting individual income and expenditures for travel, especially gasoline prices. Freight vehicle travel is also related to the economy and the indicator used to represent overall economy of the state is gross state product. Fleet fuel efficiency is based on the consideration of vehicle sales and scrappage, and technological factors as denoted by the fuel efficiency by vehicle types and model years. General procedure to compute the fleet fuel efficiency for different vehicle categories can be viewed in Figure 3.15. 


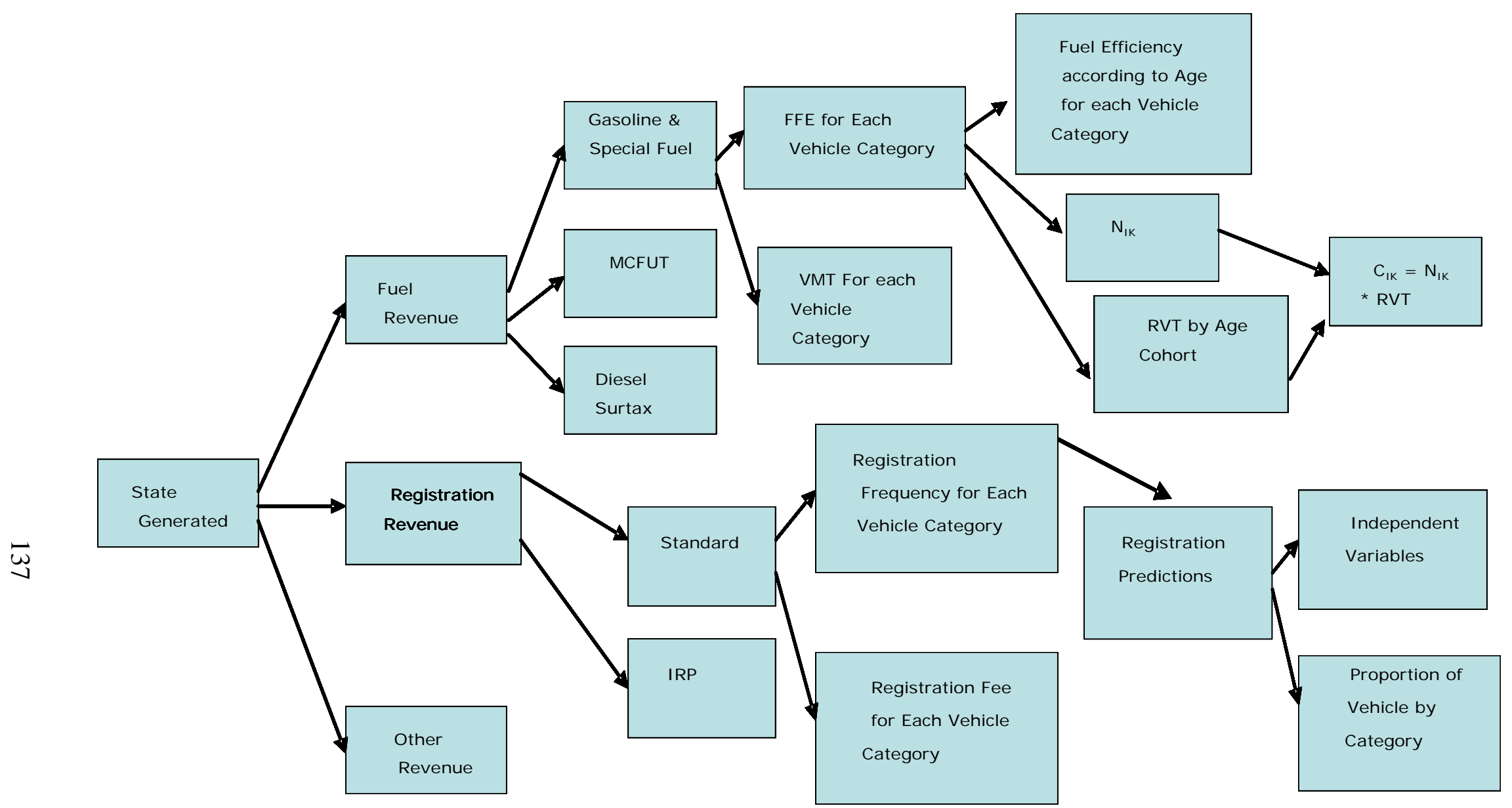

Figure 3.15 Overall Procedure for the State Generated Highway Revenue Forecasting for Indiana 


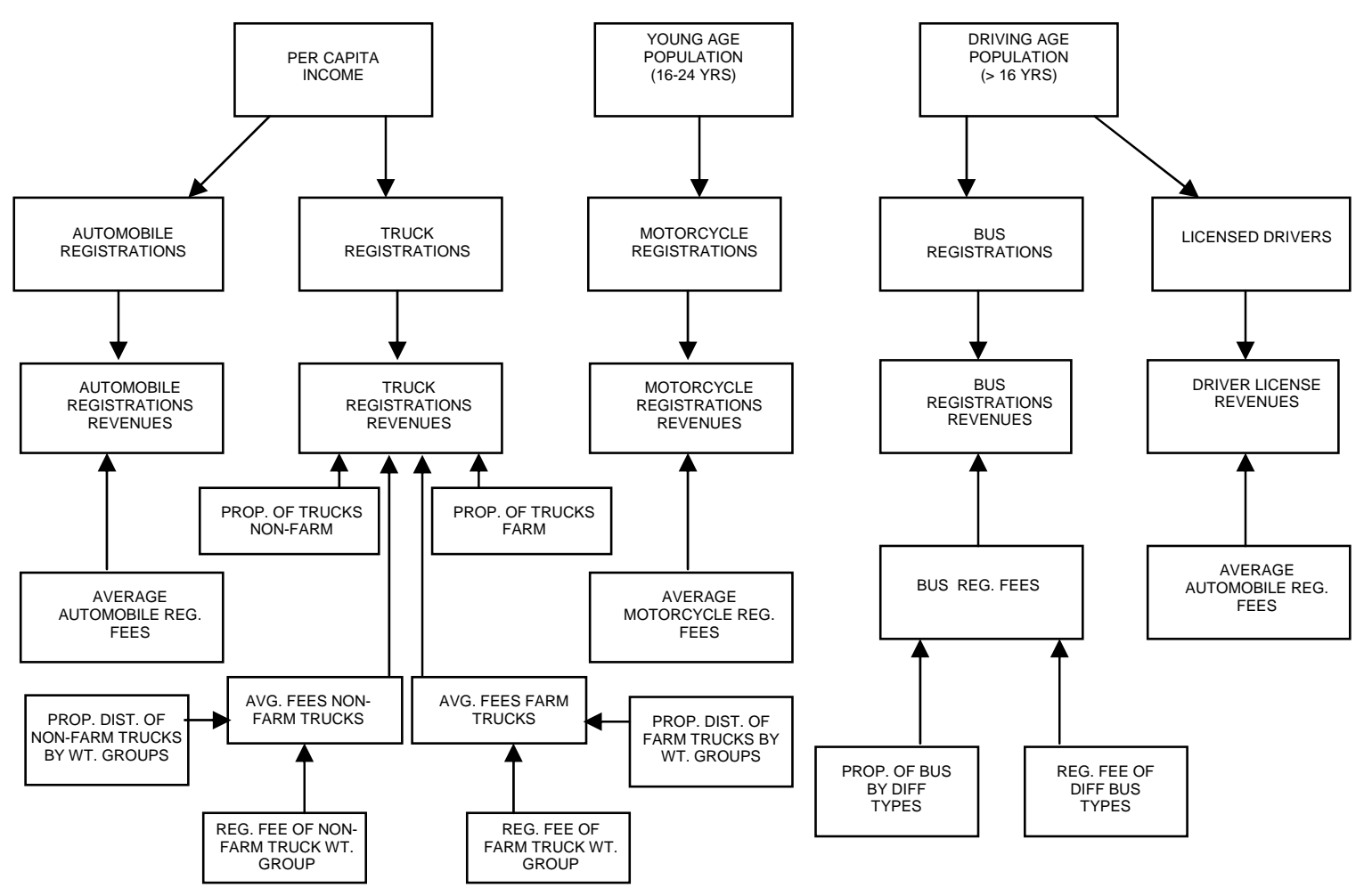

Figure 3.16 Overall Procedure to Compute Automobile, Truck, Bus, and Motorcycle Registration Revenues and Driver License Revenue 


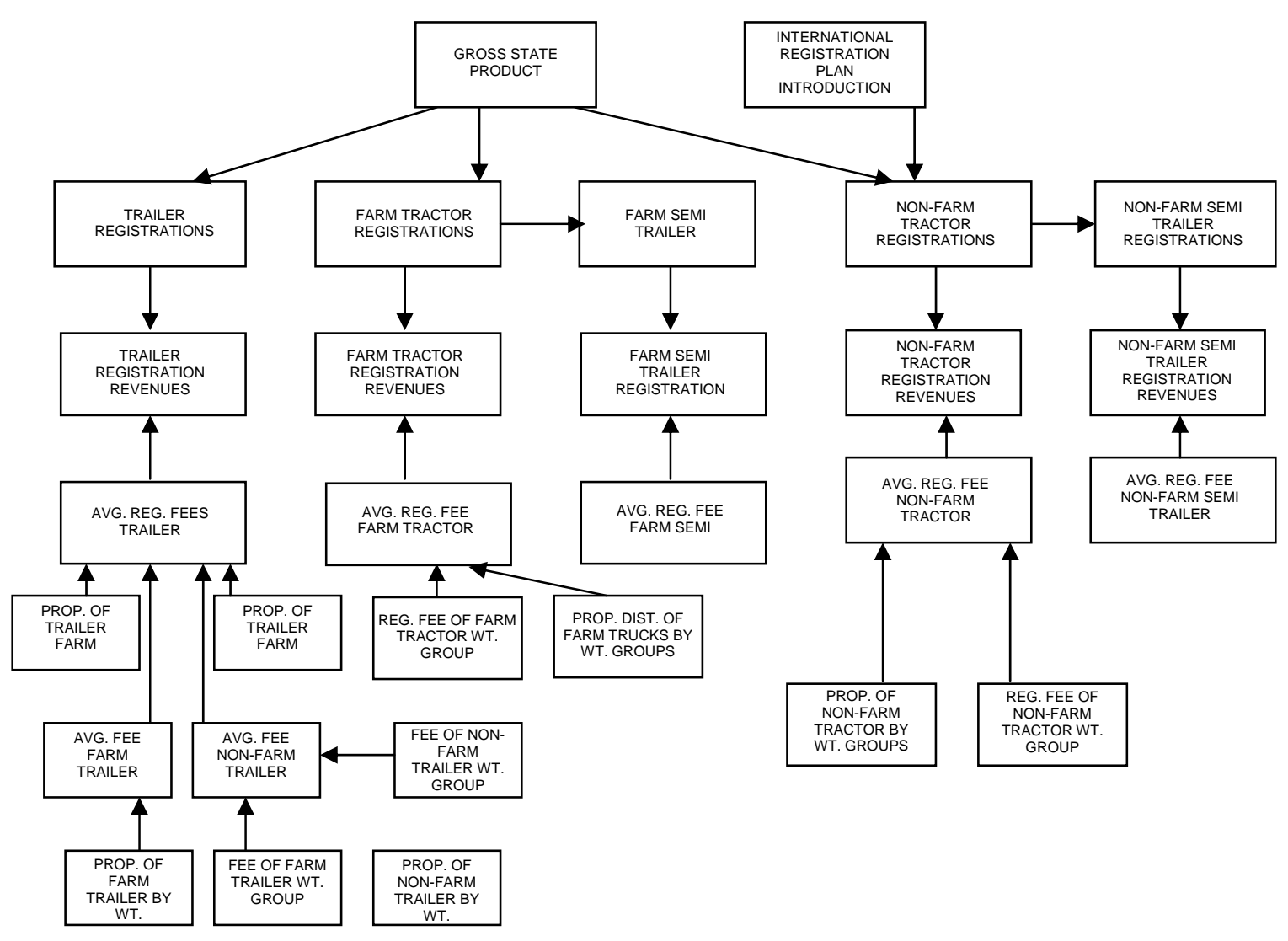

Figure 3.17 Overall Procedure to Compute Tractor, Trailer, and Semitrailer Registration Revenues 


\subsubsection{Data Requirements for Revenue Forecasting and its Use}

The sources of data items are shown in Tables 3.18 and 3.19. There are two types of data, external and internal. External data are those which are not generally available from INDOT or other related agencies, such as socio-economic data, vehicle in operation data, vehicle specific travel data, and vehicle fuel efficiency by model year and relative travel by different vehicles by model year. Internal data are those collected regularly by state agencies and include: vehicle registrations and vehicle registration fees. The data on vehicle registrations, number of licensed drivers, registration fee rates, and revenues can be obtained from the Bureau of Motor Vehicles, as well as from the annual Highway Statistics reports published by the Bureau of Transportation Statistics (BTS). Forecasting models were developed on the basis of the data available from 1993 to 2002 for vehicle registrations by different weight groups.

Economic variables used for registration related models included: Per Capita Income (PCI) (in dollars) in Indiana and Gross State Product (GSP) (in thousands of dollars). The GSP for Indiana was obtained from U.S. Department of Commerce, Bureau of Economic Analysis (2002), and Indiana Factbook. GSP values for the forecast years (2004 - 2020) were obtained using an annual growth rate of $3 \%$ as observed from historical records. Total personal income and PCI values for Indiana were obtained from Indiana Factbook, Survey of Current Business by U.S. Department of Commerce, and Woods and Poole Economics, Inc.

Demographic data such as total population, driving age population (above 16 years old), young age population (population between 15 years and 25 years old), and less than driving age population (less than 16 years old) have been considered in this study. The sources of the population data are a) Indiana State Profile by Woods and Poole Economics, Inc. b) Indiana Factbook and c) Census of Population by Bureau of Census.

The data on automobiles and trucks in operation was obtained from the R.L. Polk and Company. Vehicle stock information by age for automobiles, light duty trucks, and tractors can also be developed after determining annual vehicle sales and scrappage rates. Another feature related to vehicle travel was relative vehicle travel (RVT) for different vehicle category according to age. RVT information on automobiles was used from the earlier report (Varma et al. 1991). Information on vehicle fuel efficiency was obtained from Oak Ridge National Laboratory's Transportation Energy Book. Information on motor fuel consumption, tax rates, and motor fuel revenues was obtained from Highway Statistics, and INDOT Division of Policy and Budget. 
Table 3.18 Data Source for the Registration Related Revenue

\begin{tabular}{|c|c|}
\hline Data & Source \\
\hline $\begin{array}{l}\text { Vehicle Registrations: } \\
\text { Automobiles (Indiana) } \\
\text { Buses (Indiana): School Buses, Church Buses, } \\
\text { Intracity and Intercity Buses } \\
\text { Single Unit Trucks (Indiana): Non-Farm, Farm } \\
\text { Truck Tractors/Combination (Indiana): } \\
\text { Trucks: Farm, Non-Farm } \\
\text { Trailers (Indiana) } \\
\text { Semitrailers (Indiana) }\end{array}$ & $\begin{array}{l}\text { INDOT, Bureau of Motor Vehicles, and Annual } \\
\text { Highway Statistics reports from BTS }\end{array}$ \\
\hline $\begin{array}{l}\text { Vehicle Registrations Fees: } \\
\text { Automobiles } \\
\text { Motorcycle } \\
\text { Buses: School Buses, Church Buses, Intracity } \\
\text { and Intercity Buses } \\
\text { Trucks (graduated by gross weight): } \\
\text { Non-Farm, Farm } \\
\text { Tractors (graduated by gross weight): } \\
\text { Non-Farm, Farm } \\
\text { Trailers (graduated by gross weight): } \\
\text { Non-Farm, Farm } \\
\text { Semitrailers (graduated by gross weight): } \\
\text { Non-Farm, Farm }\end{array}$ & $\begin{array}{l}\text { INDOT, Bureau of Motor Vehicles } \\
\text { Highway Statistics }\end{array}$ \\
\hline Licensed Drivers & Highway Statistics \\
\hline $\begin{array}{l}\text { Socio-economic: } \\
\text { Population Projections } \\
\text { Total Population } \\
\text { Driving Age Population } \\
\text { Young Age (15-24 Yr) } \\
\text { Old Age Population } \\
\text { Less than Driving Age Population } \\
\text { Per Capita Income (Indiana) } \\
\text { Gross State Product (Indiana) } \\
\text { Gasoline Price (Indianapolis) } \\
\text { Consumer Price Inflation Index (CPI) } \\
\text { (National) } \\
\text { Gross National Product (GNP) Implicit Price } \\
\text { Deflator (National) }\end{array}$ & $\begin{array}{l}\text { Indiana State Board of Health } \\
\text { Indiana Business Research Center, Indiana } \\
\text { University } \\
\text { U.S. Bureau of Census } \\
\text { Woods and Poole Economics Inc., Indiana State } \\
\text { Profile } \\
\text { Indiana Factbook } \\
\text { U.S. Department of Commerce } \\
\text { Oak Ridge National Laboratory, "Transportation } \\
\text { Energy Data Book" }\end{array}$ \\
\hline
\end{tabular}


Table 3.19 Data Source for the Motor Fuel Related Revenue

\begin{tabular}{|l|l|}
\hline Data & Source \\
\hline Vehicle Travel: (Average VMT) & \\
Automobiles & \\
$\begin{array}{l}\text { Single-Unit Trucks } \\
\text { Combination Truck } \\
\text { Buses } \\
\text { Motorcycle }\end{array}$ & Annual Highway Statistics Reports from BTS \\
\hline $\begin{array}{l}\text { Vehicle Sales: } \\
\text { Automobiles } \\
\text { Trucks }\end{array}$ & \\
\hline $\begin{array}{l}\text { Vehicle Fuel Efficiency: } \\
\text { Automobiles } \\
\text { Motorcycle } \\
\text { Single-Unit Trucks } \\
\text { Combination-Trucks } \\
\text { Buses }\end{array}$ & R.L. Polk Company \\
\hline $\begin{array}{l}\text { Relative Travel by Aged Vehicles: } \\
\text { Automobiles } \\
\text { Single-Unit Trucks } \\
\text { Combination Trucks }\end{array}$ & $\begin{array}{l}\text { Oak Ridge National Laboratory, "Transportation } \\
\text { Energy Data Book 2003" }\end{array}$ \\
\hline Fuel Consumption: & Annual Highway Statistics Reports from BTS \\
$\begin{array}{l}\text { Gasoline } \\
\text { Special Fuel }\end{array}$ & \\
\hline Motor Fuel Tax Rates: & Varma et al. (1991) \\
$\begin{array}{l}\text { Gasoline } \\
\text { Special Fuel } \\
\text { Motor Carrier Fuel Use Tax } \\
\text { Diesel Surtax }\end{array}$ & \\
\hline & \\
\hline & Department of Revenue \\
\hline & Annual Highway Statistics Reports from BTS \\
\hline
\end{tabular}




\subsubsection{Vehicle Registration Forecast Equations}

Forecast equations derived by Varma et al. (1991) used a cross-sectional approach, using the data available for the period 1976-1989. In the present study, the equations were updated using recent data from 1993-2000. The resulting models indicated good agreement with the actual data. Vehicles used primarily for personal travel are automobiles, motorcycles, and a significant portion of light duty trucks. Per capita income was found to be significant for the number of registered autos and light duty trucks. Driving age population was also a significant variable for light duty truck registration. Motorcycle registration was found to be highly correlated with younger driving age population (16-24 years old). The final equations are shown below:

\section{AUTOMOBILE}

$$
\begin{gathered}
\text { AUTO }=1838684+687943 * \text { PCI } 2000 \\
\text { t-stat }(3.253) \quad(5.678) \quad\left(\mathrm{R}^{2}=0.85\right) \\
\mathrm{P}=0.001 \text { (Constant) } \quad \mathrm{P}=0.056(\mathrm{PCI})
\end{gathered}
$$

\section{LIGHT DUTY TRUCK (in 1,000,000's)}

$$
\begin{aligned}
& \text { TRUCK }=-2.63+0.056 * \text { PCI } 2000+0.813 * \text { DPOP } \\
& \begin{array}{l}
\text { t-stat }(-3.34) \quad(0.46) \\
\mathrm{P}=0.012 \text { (Constant) } \quad \mathrm{P}=0.66(\mathrm{PCI}) \quad \mathrm{P}=0.010(\mathrm{DPOP})
\end{array}
\end{aligned}
$$

\section{MOTORCYCLE (in 100,000's)}

$$
\begin{aligned}
& \text { MCYCLE }=-2.75+0.638 * \text { YPOP } \\
& \begin{array}{rrr}
\text { t-stat }(-4.68) & (6.50) & \left(\mathrm{R}^{2}=0.82\right) \\
\mathrm{P}=0.002 & \text { (Constant) } \quad \mathrm{P}=0 \text { (YPOP) }
\end{array}
\end{aligned}
$$

where,

AUTO is number of passenger cars registered in Indiana

MCYCLE is number of motorcycles registered in Indiana

LIGHT DUTY TRUCK is number of trucks registered in Indiana

YPOP is number of persons 16-24 years old in Indiana

DPOP is the driving age population in Indiana

PCI2000 is the per capita income in 2000 dollars 
Tractors are primarily used for movement of goods, and the number of tractors registered would depend on the volume of trucking activity. A good indicator of trucking activity at the state level is Gross State Product (GSP). It should be noted that registration of non-farm tractors estimated in the present study included only intrastate combination trucks, while interstate combination trucks are included in IRP computations. Since the introduction of IRP, majority of the non-farm tractors is registered under IRP, while the registration of intrastate tractors has declined. Consequently, an exponential regression model was prepared using the past historical data to forecast the non-farm intrastate tractor registration. Equations for tractors, trailers, buses and special vehicles are given below:

\title{
INTRASTATE NON-FARM TRACTOR
}

NFTRACT $=5930 * \mathrm{e}^{(-0.0171 *(t-1992))}$

$\left(\mathrm{R}^{2}=0.835\right)$

\section{FARM TRACTOR (in 1,000,000's)}

\author{
FTRACT $=-0.00716+0.00668 *$ GSP 2000 \\ t-stat (-5.39) (8.83) $\left(\mathrm{R}^{2}=0.895\right)$ \\ $\mathrm{P}=0$ (Constant $\&$ GSP 2000)
}

\section{TRAILER (in 1,000,000's)}

$$
\begin{gathered}
\text { TRAILER }=-0.184+0.336 * \text { GSP } 2000 \\
\text { t-stat }(-4.52)(14.50) \quad\left(\mathrm{R}^{2}=0.95\right) \\
\mathrm{P}=0(\text { Constant } \& \text { GSP } 2000)
\end{gathered}
$$

where,

$\mathrm{t}$ is the forecast year (greater than 1992)

NFTRACT is number of intrastate non-farm tractor registered in Indiana.

FTRACT is number for farm tractors registered in Indiana

TRAILER is number of farm and non-farm trailers registered in Indiana

GSP2000 is the gross state product in Indiana in thousands of 2000 dollars 
Bus registration frequency correlates very well with the driving age population as this group consists of common users, and has a high propensity to travel. Models were also developed for special vehicles and driving license. Good indicator for the special vehicle registration frequency is GSP. Driving age population (DPOP) is a good indicator of the driving license registration.

\section{BUS (in 1000's)}

BUSREG $=-3.66+1.83$ DPOP

t-stat $(-2.75)(6.33)\left(\mathrm{R}^{2}=0.813\right)$

$\mathrm{P}=0.025$ (Constant) $\mathrm{P}=0$ (DPOP)

\section{SPECIAL VEHICLE (in 10000's)}

$$
\begin{aligned}
& \text { SPVEH }=1.89+2.47 \text { GSP } 2000 \\
& \text { t-stat }(2.92) \quad(6.68) \quad\left(\mathrm{R}^{2}=0.83\right) \\
& \mathrm{P}=0.019 \text { (Constant) } \mathrm{P}=0(\text { GSP 2000) }
\end{aligned}
$$

\section{DRIVING LICENSE}

$$
\begin{gathered}
\mathrm{DL}=-3558360+1186233 * \mathrm{DPOP} \\
\mathrm{t}-\mathrm{stat}(-3.19) \quad(4.89)\left(\mathrm{R}^{2}=0.72\right) \\
\mathrm{P}=0.013 \text { (Constant) } \mathrm{P}=0(\mathrm{DPOP})
\end{gathered}
$$

where,

BUSREG is the number of buses registered in Indiana

DPOP is the driving age population in Indiana

SPVEH is the number of special vehicles registered in Indiana

DL is the driving license registration in Indiana

\subsubsection{Vehicle Miles of Travel Forecast Equations}

Personal vehicle travel per year is made up of annual miles traveled by a) automobiles b) light duty trucks, c) sport utility vehicles and vans, and c) motorcycles. In recent years there has been a dramatic increase in sport utility vehicle (SUV) use. Per capita income was found as a good 
indicator of vehicle miles of travel by these vehicles. The VMT forecast equation for motorcycles used motorcycle registration as the independent variable, however the $\mathrm{R}^{2}$ value observed for the model was very low and hence a separate model was developed using the historical data. The equations using the regression approach are listed below:

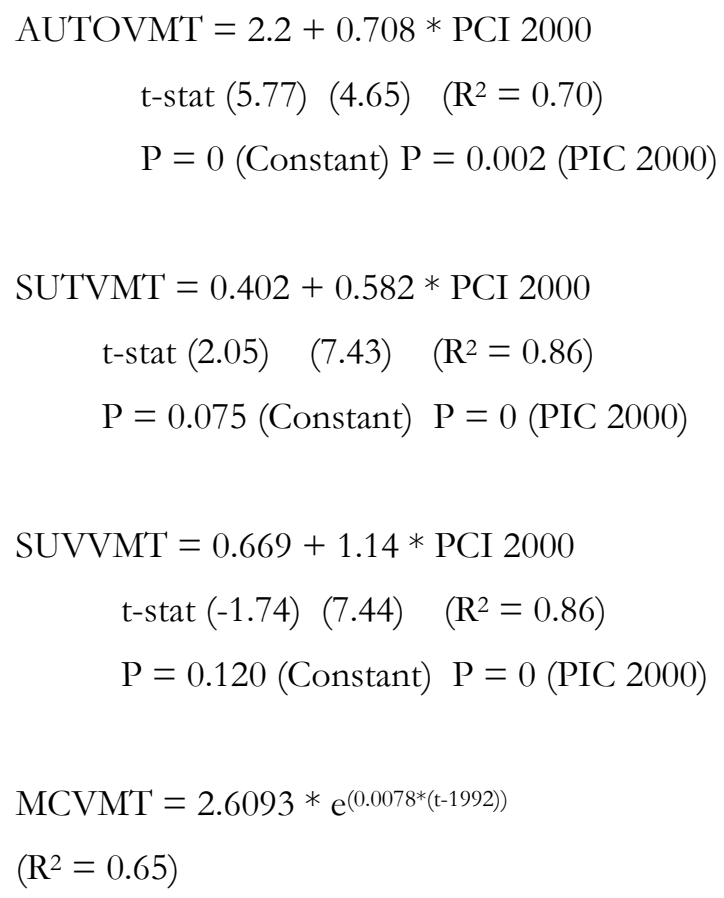

A majority of the annual commercial VMT is associated with use of tractors for goods movement. This type of vehicles is the major consumer of the special fuel such as diesel. The travel by tractors or combination vehicles is dependent on the gross state product (GSP). The total bus miles of travel was estimated on the basis of per capita income (PCI). 
COMBVMT $=0.992+1.52 *$ GSP 2000

$$
\begin{aligned}
& \text { t-stat } \quad(3.17) \quad(8.50) \quad\left(\mathrm{R}^{2}=0.90\right) \\
& \mathrm{P}=0.001 \text { (Constant) } \mathrm{P}=0(\text { GSP 2000) }
\end{aligned}
$$

BUSVMT $=0.882+0.459 *$ PCI 2000

$$
\begin{gathered}
\text { t-stat }(2.01) \quad(2.81) \quad\left(\mathrm{R}^{2}=0.63\right) \\
\mathrm{P}=0.008 \text { (Constant) } \mathrm{P}=0.003 \text { (PCI 2000) }
\end{gathered}
$$

where,

COMBVMT is the vehicle miles of travel associated with all tractors in Indiana (in 10^9)

BUSVMT is the vehicle miles of travel associated with buses in Indiana (in 10^8)

PCI 2000 is the per capita income in Indiana in 2000 dollars

GSP2000 is the gross state product in Indiana in thousands of 2000 dollars

The VMT values computed for the forecast period 2006-2020 are shown in Table 3.20. The gallonage use by different vehicle category can be computed by dividing VMT by fleet fuel efficiency.

\begin{tabular}{|l|c|c|c|c|c|c|}
\hline \multicolumn{7}{|c|}{ Table 3.20 VMT Predictions } \\
\hline Year & $\begin{array}{c}\text { AUTOVMT } \\
\text { (in 10^10) }\end{array}$ & $\begin{array}{c}\text { SUTVMT } \\
\text { (in 10^9) }\end{array}$ & $\begin{array}{c}\text { BUSVMT } \\
\text { (in 10^8) }\end{array}$ & $\begin{array}{c}\text { SUVVMT } \\
\text { (in 10^10) }\end{array}$ & $\begin{array}{c}\text { MCVMT } \\
\text { (in 10^8) }\end{array}$ & $\begin{array}{c}\text { COMBVMT } \\
\text { (in 10^9) }\end{array}$ \\
\hline $\mathbf{2 0 0 6}$ & 4.102 & 1.965 & 2.115 & 2.393 & 2.910 & 4.132 \\
\hline $\mathbf{2 0 0 7}$ & 4.123 & 1.983 & 2.129 & 2.427 & 2.933 & 4.226 \\
\hline $\mathbf{2 0 0 8}$ & 4.145 & 2.001 & 2.143 & 2.462 & 2.956 & 4.323 \\
\hline $\mathbf{2 0 0 9}$ & 4.167 & 2.019 & 2.157 & 2.497 & 2.979 & 4.423 \\
\hline $\mathbf{2 0 1 0}$ & 4.189 & 2.037 & 2.171 & 2.533 & 3.003 & 4.526 \\
\hline $\mathbf{2 0 1 1}$ & 4.210 & 2.054 & 2.185 & 2.568 & 3.026 & 4.632 \\
\hline $\mathbf{2 0 1 2}$ & 4.232 & 2.073 & 2.200 & 2.603 & 3.050 & 4.741 \\
\hline $\mathbf{2 0 1 3}$ & 4.255 & 2.091 & 2.214 & 2.639 & 3.074 & 4.854 \\
\hline $\mathbf{2 0 1 4}$ & 4.277 & 2.110 & 2.229 & 2.676 & 3.098 & 4.969 \\
\hline $\mathbf{2 0 1 5}$ & 4.300 & 2.128 & 2.243 & 2.712 & 3.122 & 5.089 \\
\hline $\mathbf{2 0 1 6}$ & 4.325 & 2.149 & 2.259 & 2.752 & 3.146 & 5.214 \\
\hline $\mathbf{2 0 1 7}$ & 4.348 & 2.168 & 2.275 & 2.790 & 3.171 & 5.341 \\
\hline $\mathbf{2 0 1 8}$ & 4.372 & 2.188 & 2.290 & 2.829 & 3.196 & 5.472 \\
\hline $\mathbf{2 0 1 9}$ & 4.397 & 2.208 & 2.306 & 2.868 & 3.221 & 5.606 \\
\hline $\mathbf{2 0 2 0}$ & 4.421 & 2.228 & 2.322 & 2.907 & 3.246 & 5.745 \\
\hline
\end{tabular}




\subsubsection{Vehicle Fleet Fuel Efficiency Determination}

The first step in determining the fleet fuel efficiency was computing the proportion of vehicles by age cohort according to vehicle category $\left(\mathrm{N}_{\mathrm{IK}}\right)$. A cohort survival method was employed using the base year vehicle stock, scrappage rates and vehicle sales data. The second step was the estimation of relative travel miles by different age cohorts, and the data developed by Varma et al. (1991) was used. Knowing the model year fuel efficiencies along with other parameters, the fuel efficiency values could be determined using the following equation:

$$
1 / \mathrm{FFE}_{\mathrm{K}}=\left(\mathrm{C}_{1 \mathrm{~K}} / \mathrm{FE}_{1 \mathrm{~K}}+\ldots . .+\mathrm{C}_{\mathrm{IK}} / \mathrm{FE}_{\mathrm{IK}}+\ldots \ldots \mathrm{C}_{\mathrm{NK}} / \mathrm{FE}_{\mathrm{NK}}\right)
$$

Where,

$\mathrm{FFE}_{K}$ is the fleet fuel efficiency of the vehicle category $K$

$F E_{I K}$ is the fuel efficiency of the vehicle category $K$ and age cohort $I$

$K$ is the vehicle category (automobile, truck, or tractor)

$I$ is the age cohort (1 to 10)

$N_{I K}$ is the proportion of vehicle category $K$ in all vehicles in age cohort I

$R V T_{I K}$ is the relative miles of travel by vehicle category $K$ in age cohort $I$

$C_{I K}$ is $N_{I K} * R V T_{I K}$

The proportion of vehicles by age cohort according to the vehicle category $\left(\mathrm{N}_{\mathrm{IK}}\right)$ was obtained by taking the ratio of vehicles in a particular age group divided by the summation of vehicles in all 10 age groups. Using a time-series analysis of the computed annual $\mathrm{N}_{\mathrm{IK}}$ values from the available data for 1993-2000, the yearly values for the forecast years (2006-2020) were estimated. These forecasted values of $\mathrm{N}_{\mathrm{IK}}$ were than used for computing the future fleet fuel efficiency values. Model year fuel efficiency values are given in Appendix M. Table 3.21 presents the computed fleet fuel efficiency values. 
Table 3.21 Estimated Fleet Fuel Efficiency (FFE) Values for Automobiles, Light Duty Trucks and Tractors (miles per gallon)

\begin{tabular}{|l|c|c|c|}
\hline Year & $\begin{array}{c}\text { Automobiles } \\
\text { FFE }\end{array}$ & $\begin{array}{c}\text { Light Duty } \\
\text { Trucks } \\
\text { FFE }\end{array}$ & $\begin{array}{c}\text { Tractors } \\
\text { FFE }\end{array}$ \\
\hline $\mathbf{2 0 0 2}$ & 23.30 & 19.54 & 4.53 \\
\hline $\mathbf{2 0 0 3}$ & 23.20 & 19.73 & 4.56 \\
\hline $\mathbf{2 0 0 4}$ & 23.15 & 19.96 & 4.63 \\
\hline $\mathbf{2 0 0 5}$ & 23.06 & 20.09 & 4.63 \\
\hline $\mathbf{2 0 0 6}$ & 25.53 & 20.73 & 4.63 \\
\hline $\mathbf{2 0 0 7}$ & 25.75 & 20.84 & 4.63 \\
\hline $\mathbf{2 0 0 8}$ & 25.94 & 21.07 & 4.64 \\
\hline $\mathbf{2 0 0 9}$ & 25.85 & 21.15 & 4.64 \\
\hline $\mathbf{2 0 1 0}$ & 25.72 & 21.18 & 4.64 \\
\hline $\mathbf{2 0 1 1}$ & 25.63 & 21.26 & 4.65 \\
\hline $\mathbf{2 0 1 2}$ & 25.54 & 21.29 & 4.65 \\
\hline $\mathbf{2 0 1 3}$ & 25.42 & 21.37 & 4.65 \\
\hline $\mathbf{2 0 1 4}$ & 25.33 & 21.40 & 4.65 \\
\hline $\mathbf{2 0 1 5}$ & 25.24 & 21.42 & 4.66 \\
\hline $\mathbf{2 0 1 6}$ & 25.12 & 21.47 & 4.66 \\
\hline $\mathbf{2 0 1 7}$ & 25.03 & 21.47 & 4.66 \\
\hline $\mathbf{2 0 1 8}$ & 24.94 & 21.47 & 4.66 \\
\hline $\mathbf{2 0 1 9}$ & 24.83 & 21.47 & 4.67 \\
\hline $\mathbf{2 0 2 0}$ & 24.80 & 21.47 & 4.67 \\
\hline & & & \\
\hline
\end{tabular}

\subsubsection{Fuel Consumption Estimation}

Fuel consumption was estimated by dividing VMT of each vehicle category by its respective fleet fuel efficiency. All fuel consumption by automobiles and motorcycles was considered to be gasoline, whereas $96 \%$ of the light duty truck and SUV fuel consumption was considered to be gasoline. Fuel consumed by tractors, buses and the remaining $4 \%$ of light duty trucks and SUVs was taken as special fuel. Fuel consumption was estimated in millions of gallons. 


\subsubsection{Highway Revenue Estimation}

\section{Registration Revenue}

Registration revenue estimates for different categories of vehicles were obtained by multiplying the estimate of registered vehicles with the registration fee for each category and type of vehicle. The latest fee structure in year 2001 was used for the entire forecast period (2006-2020). The total registration revenue was obtained as shown below:

TOTAL REGREV $=\left(\right.$ AUTO $*$ AFEE + MCYCLE $*$ MFEE + TRUCK $*$ TRUCKFEE $_{I}+$ TRACTOR $_{\mathrm{J}} *$ TRACTFEE $_{\mathrm{J}}+$ TRAILER $_{\mathrm{K}} *$ TRAILERFEE $_{\mathrm{K}}+$ BUSREG $^{*}$ BUSFEE + DRIVER LICENSE + MISC + IRP

Where,

AUTO is the number of passenger cars registered in Indiana,

AFEE is the automobile registration fee,

MCYCLE is the number of motorcycles registered in Indiana,

MFEE is the motorcycle registration fee,

TRUCK $\mathrm{I}_{\mathrm{I}}$ is the number of light duty trucks of type I registered in Indiana,

TRUCKFEE $_{I}$ is the registration fee for the truck of type I,

TRACTOR $_{\mathrm{J}}$ is the number of tractors of type $\mathrm{J}$ registered in Indiana,

TRACTFEE $_{\mathrm{J}}$ is the registration fee for the tractors of type $\mathrm{J}$,

TRAILER $_{\mathrm{K}}$ is the number of trailers of type $\mathrm{K}$ registered in Indiana,

TRAILERFEE $_{\mathrm{K}}$ is the registration fee for the trailers of type $\mathrm{K}$,

BUSREG is the number of buses registered in Indiana,

BUSFEE is the registration fee for the buses,

DRIVER LICENSE is the revenue generated related to driving license, and

MISC is the miscellaneous item which is estimated as the percentage of the total registration related revenues.

The revenue generated under International Registration Plan (IRP) was estimated using the time-series extrapolation approach with the use of historical data (1993-2002). Long-term forecasts of registration related revenues are shown in Table 3.22. The equation developed is shown below:

$\operatorname{IRP}=5 * 10^{7 *} \mathrm{e}^{(0.0099 *(\mathrm{t}-1992))}$

$\left(\mathrm{R}^{2}=0.67\right)$ 
where,

$\mathrm{t}$ is the forecast year (greater than 1992),

IRP is the annual revenue generated under International Registration Plan.

\section{Fuel Tax}

Motor fuel tax revenues for the forecast period were computed based on the current tax rates. The tax rate corresponding to gasoline or special fuel tax was multiplied by its corresponding total gallonage consumption in order to get the total revenue generated in a given year. Time series models based on the historical data for the motor carrier fuel use tax (MCFUT) and diesel surtax from the Indiana Department of Revenue were used to project the revenues from these sources. Long-term forecasts of fuel tax revenues are shown in Table 3.23. Table 3.24 presents the total longterm highway revenue forecasts for Indiana for the forecast period. 
Table 3.22 Highway Revenue Forecasts for Registration Related Revenues (2006-2020)

\begin{tabular}{|c|c|c|c|c|c|c|c|c|c|c|c|c|c|c|c|c|c|}
\hline \multirow{2}{*}{$\frac{\text { Prediction Year }}{2006}$} & \multicolumn{2}{|c|}{ Automobiles } & \multicolumn{2}{|c|}{\begin{tabular}{|c|}
$\begin{array}{c}\text { Light Duty } \\
\text { Trucks }\end{array}$ \\
\end{tabular}} & \multicolumn{2}{|c|}{ Tractors } & \multicolumn{2}{|c|}{ Trailers } & \multicolumn{2}{|c|}{ Motorcycle } & \multicolumn{2}{|c|}{ Driver Licenses } & \multicolumn{2}{|c|}{ Miscellaneous } & \multicolumn{2}{|r|}{ IRP } & \multirow{2}{*}{$\frac{\text { Total }}{\$ 312,476,490}$} \\
\hline & $\$$ & $61,748,857$ & $\$$ & $76,325,307$ & $\$$ & $8,682,371$ & $\$$ & $15,391,430$ & $\$$ & $2,888,685$ & $\$$ & $53,330,779$ & $\$$ & $38,875,888$ & $\$$ & $55,233,173$ & \\
\hline 2007 & $\$$ & $62,096,853$ & $\$$ & $78,329,697$ & $\$$ & $8,860,122$ & $\$$ & $15,969,649$ & $\$$ & $2,985,758$ & $\$$ & $54,331,558$ & $\$$ & $40,342,384$ & $\$$ & $55,782,696$ & $\$ 318,698,717$ \\
\hline 2008 & $\$$ & $62,448,305$ & $\$$ & $80,245,002$ & $\$$ & $9,046,860$ & $\$$ & $16,565,869$ & $\$$ & $3,083,964$ & $\$$ & $55,346,888$ & $\$$ & $41,864,198$ & $\$$ & $56,337,687$ & $\$ 324,938,773$ \\
\hline 2009 & $\$$ & $62,804,367$ & $\$$ & $82,071,223$ & $\$$ & $9,242,768$ & $\$$ & $17,180,623$ & $\$$ & $3,183,315$ & $\$$ & $56,377,005$ & $\$$ & $43,443,420$ & $\$$ & $56,898,200$ & $\$ 331,200,921$ \\
\hline 2010 & $\$$ & $63,162,734$ & $\$$ & $83,897,444$ & $\$$ & $9,448,113$ & $\$$ & $17,814,727$ & $\$$ & $3,283,826$ & $\$$ & $57,422,148$ & $\$$ & $45,082,213$ & $\$$ & $57,464,289$ & $\$ 337,575,494$ \\
\hline 2011 & $\$$ & $63,514,187$ & $\$$ & $85,679,123$ & $\$$ & $9,663,038$ & $\$$ & $18,468,585$ & $\$$ & $3,385,510$ & $\$$ & $58,482,560$ & $\$$ & $46,782,826$ & $\$$ & $58,036,011$ & $\$ 344,011,840$ \\
\hline 2012 & $\$$ & $63,876,011$ & $\$$ & $87,460,802$ & $\$$ & $9,887,852$ & $\$$ & $19,143,052$ & $\$$ & $3,488,379$ & $\$$ & $59,558,488$ & $\$$ & $48,547,591$ & $\$$ & $58,613,420$ & $\$ 350,575,595$ \\
\hline 2013 & $\$$ & $64,238,987$ & $\$$ & $89,242,481$ & $\$$ & $10,122,781$ & $\$$ & $19,838,765$ & $\$$ & $3,592,449$ & $\$$ & $60,650,184$ & $\$$ & $50,378,926$ & $\$$ & $59,196,575$ & $\$ 357,261,148$ \\
\hline 2014 & $\$$ & $64,608,876$ & $\$$ & $91,024,161$ & $\$$ & $10,368,011$ & $\$$ & $20,556,285$ & $\$$ & $3,697,734$ & $\$$ & $61,757,902$ & $\$$ & $52,279,344$ & $\$$ & $59,785,531$ & $\$ 364,077,844$ \\
\hline 2015 & $\$$ & $64,973,005$ & $\$$ & $92,805,840$ & $\$$ & $10,623,852$ & $\$$ & $21,296,429$ & $\$$ & $3,804,246$ & $\$$ & $62,881,903$ & $\$$ & $54,251,450$ & $\$$ & $60,380,347$ & $\$ 371,017,072$ \\
\hline 2016 & $\$$ & $65,377,615$ & $\$$ & $95,265,852$ & $\$$ & $10,897,197$ & $\$$ & $22,061,645$ & $\$$ & $3,912,002$ & $\$$ & $64,022,588$ & $\$$ & $56,297,949$ & $\$$ & $60,981,081$ & $\$ 378,815,929$ \\
\hline 2017 & $\$$ & $65,763,587$ & $\$$ & $97,259,478$ & $\$$ & $11,175,715$ & $\$$ & $22,850,547$ & $\$$ & $4,021,015$ & $\$$ & $65,179,955$ & $\$$ & $58,421,647$ & $\$$ & $61,587,791$ & $\$ 386,259,735$ \\
\hline 2018 & $\$$ & $66,153,867$ & $\$$ & $99,269,720$ & $\$$ & $11,465,679$ & $\$$ & $23,664,388$ & $\$$ & $4,131,299$ & $\$$ & $66,354,412$ & $\$$ & $60,625,457$ & $\$$ & $62,200,538$ & $\$ 393,865,360$ \\
\hline 2019 & $\$$ & $66,548,504$ & $\$$ & $101,296,717$ & $\$$ & $11,767,383$ & $\$$ & $24,503,973$ & $\$$ & $4,242,871$ & $\$$ & $67,546,238$ & $\$$ & $62,912,399$ & $\$$ & $62,819,381$ & $\$ 401,637,466$ \\
\hline 2020 & $\$$ & $66,947,545$ & $\$$ & $103,340,607$ & $\$$ & $12,081,125$ & $\$$ & $25,357,662$ & $\$$ & $4,355,744$ & $\$$ & $68,755,716$ & $\$$ & $65,283,755$ & $\$$ & $63,444,381$ & $\$ 409,566,535$ \\
\hline
\end{tabular}


Table 3.23 Forecasts for Fuel Tax Revenues (2006-2020)

\begin{tabular}{|c|c|c|c|c|c|}
\hline $\begin{array}{c}\text { Prediction } \\
\text { Year }\end{array}$ & Gasoline & $\begin{array}{c}\text { Special Fuel } \\
\text { (Diesel) }\end{array}$ & MCFUT & MCST & Total \\
\hline $\mathbf{2 0 0 6}$ & $\$ 537,197,288$ & $\$ 161,021,217$ & $\$ 2,793,021$ & $\$ 110,460,406$ & $\$ 811,471,932$ \\
\hline $\mathbf{2 0 0 7}$ & $\$ 537,147,744$ & $\$ 161,583,381$ & $\$ 2,454,256$ & $\$ 115,359,935$ & $\$ 816,545,316$ \\
\hline $\mathbf{2 0 0 8}$ & $\$ 538,888,674$ & $\$ 171,568,509$ & $\$ 2,156,581$ & $\$ 120,476,785$ & $\$ 833,090,549$ \\
\hline $\mathbf{2 0 0 9}$ & $\$ 537,760,875$ & $\$ 153,315,045$ & $\$ 1,895,010$ & $\$ 125,820,596$ & $\$ 818,791,526$ \\
\hline $\mathbf{2 0 1 0}$ & $\$ 538,694,432$ & $\$ 162,080,545$ & $\$ 1,665,165$ & $\$ 131,401,434$ & $\$ 833,841,576$ \\
\hline $\mathbf{2 0 1 1}$ & $\$ 539,497,466$ & $\$ 167,700,643$ & $\$ 1,463,198$ & $\$ 137,229,813$ & $\$ 845,891,120$ \\
\hline $\mathbf{2 0 1 2}$ & $\$ 541,591,677$ & $\$ 182,942,871$ & $\$ 1,285,727$ & $\$ 143,316,714$ & $\$ 869,136,989$ \\
\hline $\mathbf{2 0 1 3}$ & $\$ 543,756,584$ & $\$ 186,590,408$ & $\$ 1,129,782$ & $\$ 149,673,601$ & $\$ 881,150,375$ \\
\hline $\mathbf{2 0 1 4}$ & $\$ 546,863,941$ & $\$ 191,275,872$ & $\$ 9992,751$ & $\$ 156,312,452$ & $\$ 895,445,016$ \\
\hline $\mathbf{2 0 1 5}$ & $\$ 546,368,462$ & $\$ 196,203,665$ & $\$ 872,340$ & $\$ 163,245,773$ & $\$ 906,690,240$ \\
\hline $\mathbf{2 0 1 6}$ & $\$ 551,107,952$ & $\$ 194,666,885$ & $\$ 766,535$ & $\$ 170,486,624$ & $\$ 917,027,996$ \\
\hline $\mathbf{2 0 1 7}$ & $\$ 555,747,984$ & $\$ 198,545,450$ & $\$ 673,562$ & $\$ 178,048,648$ & $\$ 933,015,644$ \\
\hline $\mathbf{2 0 1 8}$ & $\$ 561,851,778$ & $\$ 216,103,780$ & $\$ 551,866$ & $\$ 185,946,089$ & $\$ 964,493,513$ \\
\hline $\mathbf{2 0 1 9}$ & $\$ 568,393,787$ & $\$ 190,092,178$ & $\$ 520,079$ & $\$ 194,193,825$ & $\$ 953,199,869$ \\
\hline $\mathbf{2 0 2 0}$ & $\$ 573,892,001$ & $\$ 194,449,936$ & $\$ 456,999$ & $\$ 202,807,393$ & $\$ 971,606,329$ \\
\hline
\end{tabular}

Table 3.24 Total Highway Revenue Forecasts for the Period (2006-2020)

\begin{tabular}{|c|cc|}
\hline Prediction Year & \multicolumn{2}{|c|}{$\begin{array}{c}\text { Total Revenues } \\
\text { (All Sources) }\end{array}$} \\
\hline $\mathbf{2 0 0 6}$ & $\$$ & $1,181,848,422$ \\
\hline 2007 & $\$$ & $1,193,144,032$ \\
\hline 2008 & $\$$ & $1,215,929,322$ \\
\hline $\mathbf{2 0 0 9}$ & $\$$ & $1,207,892,447$ \\
\hline $\mathbf{2 0 1 0}$ & $\$$ & $1,229,317,071$ \\
\hline $\mathbf{2 0 1 1}$ & $\$$ & $1,247,802,960$ \\
\hline $\mathbf{2 0 1 2}$ & $\$$ & $1,277,612,584$ \\
\hline $\mathbf{2 0 1 3}$ & $\$$ & $1,296,311,523$ \\
\hline $\mathbf{2 0 1 4}$ & $\$$ & $1,317,422,860$ \\
\hline $\mathbf{2 0 1 5}$ & $\$$ & $1,335,607,313$ \\
\hline $\mathbf{2 0 1 6}$ & $\$$ & $1,353,743,924$ \\
\hline $\mathbf{2 0 1 7}$ & $\$$ & $1,377,175,378$ \\
\hline $\mathbf{2 0 1 8}$ & $\$$ & $1,416,258,872$ \\
\hline $\mathbf{2 0 1 9}$ & $\$$ & $1,412,737,332$ \\
\hline $\mathbf{2 0 2 0}$ & $\$$ & $1,439,072,864$ \\
\hline
\end{tabular}

Note: Apart from registration and fuel revenues, $\$ 57.9$ Million of revenue generated in Year 2003 from permits and miscellaneous was added as a constant amount for every year. 


\subsubsection{Highway Revenue Disbursement}

The forecasted revenues were allocated to state and local agencies according to the currently used formulas set by the legislature as described in Section 3.1.1. The disbursement of the revenues in various highway accounts is shown in Table 3.25 for projection years (2006-2020). It can be noted

that the state agency share of the 15-year (2006-2020) total state generated revenues is expected to be $\$ 10.07$ billion, while the local share would be $\$ 7.2$ billion during the same period. 
Table 3.25 Disbursement of Revenues in Major Highway Accounts

\begin{tabular}{|c|c|c|c|c|c|c|c|c|c|c|c|c|c|c|}
\hline \multirow{2}{*}{$\frac{\text { Prediction Year }}{2006}$} & \multicolumn{2}{|r|}{ MVHA } & \multicolumn{2}{|r|}{ HRSF } & \multicolumn{2}{|r|}{ SD } & \multicolumn{2}{|r|}{ MCRF } & \multicolumn{2}{|c|}{ SHRCIF } & \multicolumn{2}{|r|}{ SHF } & \multicolumn{2}{|r|}{ LOCAL } \\
\hline & $\$$ & $814,194,813$ & $\$$ & $132,173,027$ & $\$$ & $50,000,000$ & $\$$ & $9,941,437$ & $\$$ & $59,682,619$ & $\$$ & $601,707,269$ & $\$$ & $433,827,275$ \\
\hline 2007 & $\$$ & $823,491,507$ & $\$$ & $132,303,938$ & $\$$ & $50,000,000$ & $\$$ & $10,382,394$ & $\$$ & $59,677,114$ & $\$$ & $608,333,194$ & $\$$ & $438,252,906$ \\
\hline 2008 & $\$$ & $841,020,543$ & $\$$ & $135,138,613$ & $\$$ & $50,000,000$ & $\$$ & $10,842,911$ & $\$$ & $59,870,532$ & $\$$ & $621,124,729$ & $\$$ & $447,862,908$ \\
\hline 2009 & $\$$ & $835,827,540$ & $\$$ & $130,356,031$ & $\$$ & $50,000,000$ & $\$$ & $11,323,854$ & $\$$ & $59,745,233$ & $\$$ & $617,812,115$ & $\$$ & $443,208,006$ \\
\hline 2010 & $\$$ & $852,325,479$ & $\$$ & $132,728,866$ & $\$$ & $50,000,000$ & $\$$ & $11,826,129$ & $\$$ & $59,848,951$ & $\$$ & $629,995,813$ & $\$$ & $452,081,159$ \\
\hline 2011 & $\$$ & $866,567,117$ & $\$$ & $134,289,981$ & $\$$ & $50,000,000$ & $\$$ & $12,350,683$ & $\$$ & $59,938,168$ & $\$$ & $640,715,405$ & $\$$ & $459,521,397$ \\
\hline 2012 & $\$$ & $889,027,809$ & $\$$ & $138,507,600$ & $\$$ & $50,000,000$ & $\$$ & $12,898,504$ & $\$$ & $60,170,835$ & $\$$ & $657,422,558$ & $\$$ & $472,091,033$ \\
\hline 2013 & $\$$ & $903,082,894$ & $\$$ & $139,840,288$ & $\$$ & $50,000,000$ & $\$$ & $13,470,624$ & $\$$ & $60,411,356$ & $\$$ & $668,480,882$ & $\$$ & $479,415,702$ \\
\hline 2014 & $\$$ & $918,730,015$ & $\$$ & $141,615,647$ & $\$$ & $50,000,000$ & $\$$ & $14,068,121$ & $\$$ & $60,756,584$ & $\$$ & $680,924,280$ & $\$$ & $487,739,665$ \\
\hline 2015 & $\$$ & $932,719,126$ & $\$$ & $142,751,286$ & $\$$ & $50,000,000$ & $\$$ & $14,692,120$ & $\$$ & $60,701,536$ & $\$$ & $691,691,443$ & $\$$ & $494,798,334$ \\
\hline 2016 & $\$$ & $945,916,678$ & $\$$ & $143,288,329$ & $\$$ & $50,000,000$ & $\$$ & $15,343,796$ & $\$$ & $61,228,093$ & $\$$ & $702,878,287$ & $\$$ & $501,503,524$ \\
\hline 2017 & $\$$ & $962,913,545$ & $\$$ & $145,159,877$ & $\$$ & $50,000,000$ & $\$$ & $16,024,378$ & $\$$ & $61,743,601$ & $\$$ & $716,872,474$ & $\$$ & $510,589,450$ \\
\hline 2018 & $\$$ & $991,342,990$ & $\$$ & $150,735,884$ & $\$$ & $50,000,000$ & $\$$ & $16,735,148$ & $\$$ & $62,421,733$ & $\$$ & $739,015,735$ & $\$$ & $526,796,201$ \\
\hline 2019 & $\$$ & $987,681,193$ & $\$$ & $145,504,587$ & $\$$ & $50,000,000$ & $\$$ & $17,477,444$ & $\$$ & $63,148,550$ & $\$$ & $739,682,246$ & $\$$ & $523,080,883$ \\
\hline 2020 & $\$$ & $1,006,516,030$ & $\$$ & $147,662,742$ & $\$$ & $50,000,000$ & $\$$ & $18,252,665$ & $\$$ & $63,759,401$ & $\$$ & $755,523,699$ & $\$$ & $533,206,828$ \\
\hline \multicolumn{11}{|c|}{ Total } & & $, 072,180,129$ & $\$$ & $7,203,975,271$ \\
\hline
\end{tabular}

MVHA - Motor Vehicle Highway Account

HRSF - Highway Road and Street Fund

SD - Special Distribution

MCRF - Motor Carrier Regulation Fund

SHRCIF - State Highway and Road Construction and Improvement Fund

SHF - State Highway Fund

LOCAL - Local Agencies 


\subsubsection{Validation of the Model Results}

A comparison of long-term registration related revenues with actual values was made for years 2001 and 2002, as shown in Table 3.26. The forecasts for various categories were within the range of $\pm 10 \%$, whereas the total registration revenue estimate was within $-1.6 \%$. Similarly, a comparison for motor fuel related revenues for years 2001 and 2002 is shown in Table 3.27. The values estimated for each category were also obtained within the range of $\pm 10 \%$, whereas the total motor fuel revenue estimation was within $+2.5 \%$ difference. It should be noted that the revenue generated by Motor Carrier Fuel Use Tax (MCFUT) was included in the total motor fuel revenue prediction. Validation results for the overall state level revenue showed that the estimated value was within $-0.65 \%$ and $-0.91 \%$ difference, as compared to the actual values for year 2001 and 2002, respectively.

\begin{tabular}{|c|c|c|c|c|c|c|}
\hline \multicolumn{7}{|c|}{$\begin{array}{l}\text { Table 3.26 Validation of the Model Using Actual } \\
\text { Registration Revenue for Years } 2001 \text { and } 2002 \text { (2002\$ value in Millions) }\end{array}$} \\
\hline & \multicolumn{3}{|c|}{2001} & \multicolumn{3}{|c|}{2002} \\
\hline & Model & Actual & $\%$ Difference & Model & Actual & $\%$ Difference \\
\hline Automobiles & $\$ 60.0$ & $\$ 62.4$ & $-3.8 \%$ & $\$ 60.4$ & $\$ 62.9$ & $-4.0 \%$ \\
\hline Light Duty Trucks & $\$ 66.7$ & $\$ 66.1$ & $0.9 \%$ & $\$ 68.5$ & $\$ 68.4$ & $0.1 \%$ \\
\hline Tractors + Trailers & $\$ 20.6$ & $\$ 23.1$ & $-10.8 \%$ & $\$ 21.8$ & $\$ 23.5$ & $-7.2 \%$ \\
\hline Motorcycles & $\$ 2.47$ & $\$ 2.51$ & $-1.6 \%$ & $\$ 2.7$ & $\$ 2.6$ & $2.8 \%$ \\
\hline Driver Licenses & $\$ 16.4$ & $\$ 17.5$ & $-6.3 \%$ & $\$ 16.7$ & $\$ 15.7$ & $6.4 \%$ \\
\hline IRP & $\$ 52.6$ & $\$ 52.6$ & $0.0 \%$ & $\$ 53.1$ & $\$ 53.1$ & $0.0 \%$ \\
\hline Miscellaneous & $\$ 30.2$ & $\$ 32.3$ & $-6.5 \%$ & $\$ 30.7$ & $\$ 33.5$ & $-8.4 \%$ \\
\hline Titles & $\$ 32.2$ & $\$ 28.7$ & $12.2 \%$ & $\$ 32.7$ & $\$ 31.5$ & $3.8 \%$ \\
\hline Total REGREV & $\$ 281.1$ & $\$ 285.2$ & $-1.4 \%$ & $\$ 286.5$ & $\$ 291.2$ & $-1.6 \%$ \\
\hline
\end{tabular}

Table 3.27 Validation of the Model Using Actual

Motor Fuel Revenues for Years 2001 and 2002 (2002 \$ Value in Millions)

\begin{tabular}{|c|c|c|c|c|c|c|}
\hline & \multicolumn{3}{|c|}{$\mathbf{2 0 0 1}$} & \multicolumn{3}{|c|}{$\mathbf{2 0 0 2}$} \\
\hline & Model & Actual & \% Difference & Model & Actual & \% Difference \\
\hline Gasoline & $\$ 524.7$ & $\$ 487.3$ & $7.7 \%$ & $\$ 527.4$ & $\$ 491.4$ & $7.3 \%$ \\
\hline Surcharge & $\$ 88.9$ & $\$ 86.6$ & $2.7 \%$ & $\$ 92.9$ & $\$ 95.5$ & $-2.7 \%$ \\
\hline Special Fuel & $\$ 145.8$ & $\$ 162.5$ & $-10.2 \%$ & $\$ 148.5$ & $\$ 165.0$ & $-10.6 \%$ \\
\hline $\begin{array}{c}\text { Total Motor Fuel Revenue } \\
\text { (includes MCFUT) }\end{array}$ & $\$ 761.1$ & $\$ 741.9$ & $2.5 \%$ & $\$ 764.7$ & $\$ 755.2$ & $1.3 \%$ \\
\hline
\end{tabular}




\subsubsection{Sensitivity of the Forecasts}

Major independent variables/parameters that can change the revenue projections include: vehicle registrations, vehicle miles of travel, vehicle sales, registration fees of different vehicle category, proportion of vehicles by weight for different category of vehicles, gasoline tax rate, special fuel tax rate, fuel efficiency of the vehicle, increase in PCI and increase in GSP. A small change in any of the major variables can have an impact on the revenues predicted for a particular forecast year. For example, an increase in the gasoline tax by 1 cent per gallon could increase the revenue by $5.5 \%$ with a total increase of revenue by about $\$ 30$ million. Similarly, the revenue sensitivity of other variables can also be identified. 


\section{CHAPTER 4: GAP ANALYSIS FOR STATE AND LOCAL AGENCIES}

\subsection{State Highway Funding Gap Analysis}

In order to analyze state agency funding gaps, six scenarios were considered, as shown in Table 4.1. Each scenario consists of an expected level of state and federal funding. For example, Scenario 1 includes the six-year average annual funding for the State Highway Fund of $\$ 593.5$ million and the six-year average annual Federal Aid Funding of $\$ 423$ million held constant over 15 years.

Table 4.1 indicates expected revenue gaps associated with the six scenarios. If the six-year average annual revenue for the State Highway Fund and Federal Aid are used (Scenario 1), the total 15 -year projected revenue would be $\$ 15.2$ billion. With the state highway agency need in the range of $\$ 29.8$ to $\$ 30.2$ billion, the annual funding gap would be $\$ 0.97$ to $\$ 1.0$ billion. If the maximum projected revenues for both SHF and Federal Aid were used (Scenario 6), the total revenue is 16.87 billion dollars. With $\$ 16.87$ billion as the projected revenue, the funding gap would be $\$ 0.86$ to $\$ 0.89$ billion. Scenario 6 includes state generated revenues obtained from forecasting models INDOTREV and a constant amount of Federal Aid of $\$ 6.8$ billion for 15 years considering the 3-year average. Adding the 6-year average of the federal aid projected for 15 years to the state generated revenues obtained from forecasting models, Scenario 5 can be created which gives the value of 15 year revenue

for the state agency to be $\$ 16.37$ billion. Based on this value the funding gap would be in a range of $\$ 0.90$ to $\$ 0.92$ billion. 
Table 4.1 Fifteen-year State Agency Funding Gap Using HERS-ST Analysis (in Billions of 2002 \$)

Total 15-year State Highway Needs

\section{State Generated Revenue (SHF)}

6-year average held

No revenue increases

Model Results

\section{Projected Federal Aid Revenue}

6-year average held

3 -year average held

Revenue Scenarios

Scenario 1: SHF 6-year avg. + Fed Aid 6-year avg.

Scenario 2: SHF no increase + Fed. Aid 6-year avg.

Scenario 3: SHF 6-year avg. + Fed Aid 3-year avg.

Scenario 4: SHF no increase + Fed. Aid 3-year avg.

Scenario 5: Model Results + Fed. Aid 6-year avg.

Scenario 6: Model Results + Fed. Aid 3-year avg.
Needs

$\$ 29.8$ to $\$ 30.2$

Projected Revenue

$\$ 8.9$

$\$ 7.1$

$\$ 10.07$

$\$ 6.3$

$\$ 6.8$

15-year Revenue Total
$\$ 15.20$
$\$ 13.40$
$\$ 15.70$
$\$ 13.90$
$\$ 16.37$
$\$ 16.87$

Funding Gap Analysis

$\$ 14.6$ to $\$ 15.0$

$\$ 0.97$ to $\$ 1.00$

$\$ 16.4$ to $\$ 16.8$

$\$ 1.09$ to $\$ 1.12$

$\$ 14.1$ to $\$ 14.5$

$\$ 0.94$ to $\$ 1.04$

$\$ 15.9$ to $\$ 16.3$

$\$ 1.06$ to $\$ 1.09$

$\$ 13.43$ to $\$ 13.83$

$\$ 0.90$ to $\$ 0.92$

$\$ 12.93$ to $\$ 13.33$

$\$ 0.86$ to $\$ 0.89$

The total 15-year state highway agency needs using the manual analysis method were $\$ 18.8$ to $\$ 19.7$ billion. It must be noted that the needs for this method do not include costs for shoulder work or alignment improvements over the 15 -year period. The consideration of these costs would move the estimates closer to those found in the HERS-ST method. Consequently, no separate funding gap analysis was prepared for the needs analysis using manual method. 


\subsection{Local Highway Funding Gap Analysis}

The total needs estimated for the study period (2006-2020) necessary to maintain and upgrade all Indiana local roads attain approximately $\$ 28.1$ to $\$ 30.4$ billion, including capacity needs and overhead costs. The average monetary needs per year represent approximately $\$ 1.95$ billion per year. On the basis of the most optimistic method of revenue projection adopted, $\$ 15.7$ billion of revenue are expected from the current revenue sources which represent approximately $\$ 1.04$ billion per year. The resulting funding gap is therefore estimated at $\$ 12.4$ to $\$ 14.7$ billion, representing $\$ 0.83$ to $\$ 0.98$ billion of shortfall per year. Table 4.2 summarizes the estimated needs, the projected revenue and the resulting gap for the study period.

The recent needs assessment of local roads in Indiana (LTAP 2001) estimated the funding shortfall at approximately $\$ 2$ billion as the backlog or immediate needs and determined that $\$ 0.46$ billion were necessary to maintain the system each year. A total of approximately $\$ 9.5$ billion would therefore be required for the study period (2002 dollars). However, the earlier estimation did not include the capacity needs determined in the present study which represent $\$ 2.2$ billion. If the capacity needs are excluded from the shortfall established in the present study, the gap would be estimated at $\$ 10.2$ to $\$ 12.5$ billion. 
Table 4.2: Fifteen-year Local Agencies Funding Gap Analysis (in Billions of 2002 \$)

\begin{tabular}{lc}
\hline & \\
\hline Categories of Local Highway Needs & \\
Total 15-year Local Highway Needs & $\$ 28.1$ to $\$ 30.4$ \\
Average Needs per year & $\$ 1.9$ to $\$ 2.03$ \\
\cline { 2 - 2 } Revenue Source & Projected Revenue (in billion) \\
\cline { 2 - 2 } $\begin{array}{l}\text { MVHA and LRSF } \\
\text { Federal Aid }\end{array}$ & $\$ 7.4$ to $\$ 9.1$ \\
Additional Revenue & $\$ 2.2$ \\
Total Revenue & $\$ 4.2$ \\
Average Revenue per year & $\$ 13.8$ to $\$ 15.7$ \\
& $\$ 0.92$ to $\$ 1.04$ \\
& \\
Total Gap (based on most optimistic projected & Gap Analysis (in billion) \\
revenue) & $\$ 12.4$ to $\$ 14.7$ \\
Average Gap Range per year & $\$ 0.83$ to $\$ 0.98$
\end{tabular}

\subsection{Gap Reduction Scenarios}

This section presents possible adjustments to current highway user fees that could be used to meet the funding gap.

\subsubsection{Increase in State Gasoline Tax}

The total annual revenue gap for both the state and local highway systems would be about $\$ 1.7$ to $\$ 1.9$ billion. In FY 2003 approximately 3.1 billion gallons of gasoline were consumed in the state of Indiana. If the entire revenue gap was to be covered by an increase in the gasoline tax, a 56 cent to 64 cent increase on the current 18 cents per gallon would be required assuming there is no elasticity of gasoline demand with respect to price. The Federal gasoline tax rate is currently 18.4 cents per gallon and the U.S. average gasoline tax rate is 20.4 cents per gallon. A gasoline tax rate of 74 cents per gallon is highly unrealistic and the entire funding gap cannot be covered solely by increasing the gasoline tax.

\subsubsection{Increase in State Diesel Tax}

Currently, two separate user fees are associated with diesel fuels, 16 cents per gallon motor carrier fuel use tax (MCFUT) and 11 cents per gallon motor carrier surtax. These two user fees combine for 27 cents per gallon total user fee on diesel fuel. The consumption associated with these 
two user fees in FY 2003 was approximately 830 million gallons. Even if half of the 1.7 billion-dollar funding gap were to be covered by increases to the diesel fuel user fees only, then a 3-fold increase on the current 27 cents per gallon would be required. This would represent a need for an additional 100 cents per gallon, for a total of $\$ 1.27$ per gallon. The current Federal diesel tax rate is 24.4 cents per gallon and the U.S. average diesel tax rate is 23.9 cents per gallon. A diesel tax rate of $\$ 1.27$ per gallon is therefore not realistic.

\subsubsection{Combination of Gasoline and Diesel Tax Increases}

If an increase in both the gasoline tax rate and the diesel tax rate were used to share the burden of reducing a part of the revenue gap, more realistic tax rates may be obtained. However, in order to close only half of the $\$ 1.7$ billion gap, a gasoline tax rate hike of about 14 cents per gallon and a diesel tax rate hike of about 50 cents per gallon would be necessary, assuming the gap is shared equally by the gasoline and diesel taxes.

\subsubsection{Tolling}

The possibility of using tolls as a source of financing has been mentioned in recent years. If tolls were used on 100 percent of the state-controlled roads and the 2002 VMT level of 40 billion were maintained, then 5.1 cents per mile toll would be needed to cover 1.5 billion-dollar highway funding gap. However, it is not practical that all state-controlled roads can be tolled. If only interstates could be tolled, then the user cost would be 17 cents per mile which is much higher than most toll rates in the country. Also, one should consider the cost of tolling which can be expected to be substantial.

\subsubsection{Gap Reduction Scenarios for Local Roads}

Local option transportation taxes have become a key tool for financing new transportation infrastructure and services in many metropolitan areas in the country (Goldman and Wachs, 2003). Such taxes have been adopted in one form or another in at least 46 states. Local governments can choose between a wide range of different taxation options. Depending on the type of tax chosen, the outcome can vary considerably in terms of the generated revenue, the fairness of the tax to different tax payers, and its appropriateness as a funding source for transportation projects. Sales taxes are the most commonly used revenue source for major new transportation initiatives. 


\subsubsection{Local Fuel Taxes}

Local taxes on motor vehicle fuels such as gasoline are authorized in 15 states, and have been adopted in ten. In states where they are in widespread use (Alabama, Florida, Hawaii, Nevada), the funds support the operating budgets of local highway agencies. In suburban Northern Virginia, a regional sale tax on gasoline funds a variety of public transit and road investment. In Alabama and New Mexico, revenues from local gasoline taxes are often used for non-transportation purposes, such as education or health care. In most states, local fuel taxes have not been adopted at high enough levels to fund major new capital. However some states such as Florida rely upon local fuel tax option to an unusually high degree. Every county in the state of Florida has adopted a local option fuel tax, at rates ranging between $1 \phi$ and $11 \phi$ per gallon. The counties vary in how they use their fuel tax revenues, some dedicate them entirely to transit, and others invest in a mix of transit, roads, and streets. Indiana does not have a local option fuel tax.

\subsubsection{Local Vehicle Taxes}

A wide range of taxes on motor vehicles is used to fund transportation investments around the country. The most important varieties are license or registration fees, which tend to be either flat fees based on vehicle type, or variable fees based on a vehicle's value. Thirty-three states authorize some form of local vehicle taxes. Many of these allow these taxes to be used as a general revenue source. Twenty-two states are identified as having at least some local option vehicle taxes earmarked for transportation-related purposes. In most of these, the revenues are intermingled with other revenue streams in a county road fund or the budget of a transit agency. As with local option fuel taxes, these taxes typically fund pay as- you-go programs of routine maintenance and operations work. However, a few places do earmark these taxes for specific, major capital investments. In some states, the revenues are earmarked for transportation-related environmental or public safety programs.

\subsubsection{Local Option Highway User Tax (LOHUT)}

The Local Option Highway User Tax (LOHUT) is known as Wheel Tax in Indiana. It passed Indiana's General Assembly in 1980 and is the only optional tax specifically for road funding. LOHUT is composed of two taxes that must be adopted at the same time, the excise surtax and the wheel tax: 
- The excise surtax is an additional fee placed on the vehicle excise tax paid annually at the time of registration by owners of automobiles, motorcycles, and trucks in the 11,000 pound weight class or lighter. The excise surtax may be either:

0 A rate between 2 and 10 percent of the excise tax values.

0 One specific amount between $\$ 7.50$ and $\$ 25.00$.

- The wheel tax is a tax comprised between $\$ 5$ and $\$ 40$ per vehicle placed on all vehicles not subject to the excise surtax. The wheel tax vehicle categories are:

o Buses (except church buses)

o Recreational vehicles

o Semi-Trailers

o Tractors

o Trailers

o Trucks over the $11,000-1 \mathrm{~b}$ class

Wheel Tax exceptions include vehicles owned by the state, political subdivisions, religious organizations, or nonprofit youth organizations. There may be different rates for each vehicle class.

For LOHUT to be imposed, the County Council must, after giving public notice, concurrently pass an ordinance adopting the Excise Surtax and the Wheel Tax. If there is not a unanimous vote, then it must pass at two different meetings. If LOHUT is passed after December 31 and before July 1 , then it will be collected starting January of the following year. If passed after June 30 , then it will be collected two years later.

Except for the BMV administrative fee of 15 cents per vehicle, all revenues generated by a LOHUT must remain within the county for use on county roads and city and town streets. In 2001, revenues from LOHUT accounted for $\$ 17,522,945$ representing 26 counties. According to an estimation made by LTAP, if all the counties had adopted LOHUT in 2001, a total of $\$ 106,271,036$ would have been generated if all the counties had adopted LOHUT.

Presently 36 counties have adopted LOHUT. The state's largest county, Marion County, adopted this tax in 1992. Tippecanoe County is one of the counties that have recently passed LOHUT, however the wheel tax is not well received by county residents who feel there are too many taxes on citizens. 


\subsubsection{Property Taxes}

Property taxes are the most important and universal local revenue source in the United States. Their primary function is to fund services that are administered at the most local levels of government, such as street maintenance and public transit operations. While local property taxes are authorized in all 50 states, there are many different traditions as to whether transportation activities receive an explicit earmark as part of an area's total property tax rate. Because there are few centralized sources of data on property taxation, we were only able to get a partial picture of how these taxes are used around the country. But, at least 17 states have dedicated property taxes for street and road investments, and 14 have dedicated property taxes for public transit services. Several states have highly differentiated local road systems, with each functional level supported by a different property tax.

In Indiana, counties generally may not use property taxes to fund highway construction or maintenance, but they may adopt such taxes for bridges projects. In 2001, 89 of the 92 counties adopted bridge fund property tax levies through the Cumulative bridge fund which (statutory maximum of $\$ 0.30$ per $\$ 100.00$ per assessed valuation). In $2001, \$ 53$ million were generated by the Cumulative Bridge Fund.

\subsubsection{Sales Taxes}

Sales taxes provide local governments with an opportunity to generate substantial tax revenues that are often dedicated to transportation purposes. 33 states have authorized local option sales taxes for transportations projects. An important characteristic of the sales tax is its broad based: the total amount of retail goods and services purchased within an area. Despite some variation in this base from state to state (depending on whether or not food and services are included), it universally produces high revenues for a low marginal tax rate. In a metropolitan county, a sale tax of just one half of one percent can generate revenue of $\$ 50-75$ per capita.

In Indiana, there are no transportation related sales taxes. Illinois, one of the neighboring states, has adopted a transit dedicated sale tax which generates almost $\$ 59$ per capita of revenue. In 2002, Miami Dade County approved $1 / 2 \%$ sales tax to expand mass transit and Las Vegas approved $1 / 4 \%$ sales tax increase for transportation improvements. The state of Indiana collects approximately $\$ 590$ per capita annually on sales taxes. On the basis of the US census bureau population estimate, of 2003, a $1 / 2 \%$ increase in tax rate is expected to generate about $\$ 18$ million of revenue. 


\subsubsection{Income, Payroll, and Employer Taxes}

Sixteen states authorize local taxes on income (paid by individuals based on their salary), payroll (paid by employers based on the total wages they distribute), or number of workers employed at a business. Local areas have adopted payroll taxes (or income/payroll combinations) to fund transportation investments in four of these states (Indiana, Kentucky, Ohio, and Oregon), and have adopted flat, per-employee fees in Washington. These taxes generally fund programs aimed at facilitating peak-hour commutes into central cities, including public transit services, vanpools, and other projects aimed at congestion relief.

Three different local option income taxes are authorized in Indiana. The "County Adjusted Gross Income Tax" may be set at a rate up to $1 \%$ with revenues being used for general revenues or property tax relief. The "County Option Income Tax" may also be set at a rate up to $1 \%$; the revenues go to public transportation, redevelopment, general revenues, or other purposes. Only one of the two taxes may be adopted by ordinance for a county. Finally, a "County Economic Development tax" of up to $1 / 2 \%$ alone or in addition to either of the two taxes cited above. Revenues may be used for infrastructure investments, as well as other economic development activities. These taxes are based upon the individual's county of residence and employment. In 2001, approximately $\$ 64$ million generated by local option income taxes were used for transportation related purposes in Indiana counties.

\subsubsection{Other Taxes}

Other types of taxes are allowed in some states around the country. Four states use local taxes on natural resource extraction (severance taxes) to fund rural road construction and maintenance. In five states also, local transportation revenues can be derived from taxes on the sale of property (known as mortgage recording taxes or real estate transfer taxes). Many states allow local governments to tax tourists to fund infrastructure and facilities that they use heavily; local governments in at least three states are using these taxes to build major transportation projects. Finally, in a growing number of states, development impact taxes are being used to fund road construction at the metropolitan fringe. 


\section{CHAPTER 5: CONCLUSION}

\subsection{Summary of Findings}

The need for state highway infrastructure preservation and capital improvements were estimated by considering the current road and bridge conditions and projecting their condition over a 15-year horizon using the HERS-ST software, and the Indiana Bridge Management System as well as

long range capital needs. The financing needs for the state highway are in the range of about 30 billion dollars (2002 \$) for 15-year period, 2006-2020.

The present study also determined the physical and monetary needs for the preservation of Indiana's roads and bridges on the local highway system for the same 15-year period. Local highway needs, including pavements, bridges and culverts as well as safety and capacity needs are about $\$ 29$ billion.

Long term forecasts of state highway revenues by revenue source were developed in this study. The forecasting model considers six major revenue categories: registration fee, driver license fee, gasoline tax, special fuel tax, motor carrier fuel use tax, and diesel surtax. The software provides state and local shares of state generated revenues year by year for the 15-year period. A comparison of model results indicated that the revenue estimates were within $1 \%$ of the actual values for the year 2001 and 2002. On the basis of model results the 15-year revenue for the state agency from state generated sources would be about $\$ 10$ billion, while the local share would be about $\$ 7$ billion.

The best possible estimate of the 15-year state highway revenue, from both state and Federal sources is $\$ 16.87$ billion. The resulting revenue gap for the state highway agency would then be $\$ 12.93$ to $\$ 13.33$ billion for the 15 -year period or $\$ 0.86$ to $\$ 0.89$ billion annually. The projected revenue for local roads was found to be about $\$ 13.8$ to $\$ 15.7$ billion for the 15 -year period. The total resulting shortfall ranges from $\$ 12.4$ to $\$ 14.7$ billion or $\$ 0.83$ to $\$ 0.98$ billion annually.

Shortfall in funding for transportations facilities and services is not unique to the state of Indiana; nationwide many states are experiencing shortfalls in their highway system funding. To reduce the gap between infrastructure needs and available funding, the possibility of increasing existing user fees can be considered. However, the entire revenue gap cannot be realistically met using only existing user fees. A combination of fuel tax increases and tolling can be examined. In addition, possible other sources of revenue should be explored. For example, joint developments of land around interchanges can provide additional revenue for new road construction. Also, several local option transportation taxes can be considered, for the local highway system. In Indiana, parts of income and property taxes are used to finance local highway infrastructure. In addition, an increase in sales taxes can be considered as well as the adoption of local motor fuel taxes. Vehicle taxes, such as LOHUT already adopted in 36 counties, could be generalized to all counties. However, the 
magnitude of the shortfall may be very different from one county to another and adopting local taxes may also not be as effective in a county as in another one. Separate and detailed needs studies for each county should be performed in order to determine the shortfall specific to the county and also choose properly the type of tax that would more likely be voted and prove efficiency in its application for the given county.

\subsection{Implementation of the Study Results}

The present study has identified a shortfall in highway financing revenue for the 15 -year horizon. As such, policy-makers at the state and local levels can use the study results for fiscal planning and budgeting. The revenue-raising recommendations presented in this study can be a starting point for further exploration to address the funding gap identified.

\subsection{Future Work}

The present study addressed the 15-year highway financing needs for the state of Indiana, and in the process identified a number of areas that could be investigated at a future date. Future research may seek to expand the present study to answer the question of what type of user tax would be equitable and politically acceptable to taxpayers in Indiana. Additionally, TEA-21, the Federal highway bill, expired during the course of this research and a new bill has not been finalized. When a new Federal highway bill becomes law, the revenue projections associated with Federal Aid can be modified and the gap analysis revised.

The use of local option transportation taxes needs further study. The pattern observed nationwide is a gradual shift toward sales tax and away from user taxes. This trend in local transportation finance has been an ongoing rise in the use of local option sales taxes in both road and transit finance. Comparatively, the number of areas using gasoline taxes and vehicles taxes which represent user taxes has remained roughly constant. In many states, user taxes have been declining; some governments (Florida) have even used sales tax revenues to eliminate tolls. However, the idea remains strong that transportation investments should be paid by those who use them. Identifying and taxing user groups who benefit from particular transportation projects should be the principal concern for local transportation officials. Other areas have also adopted taxes targeted at tourism, mining and real estate development to fund transportation infrastructure that serve these industries. Local option transportation taxes are various and could be an important component in meeting the increasing shortfall in local transportation funding. 


\section{LIST OF REFERENCES}

AASHTO (1990) A Policy on Geometric Design of Highways and Streets. Washington, D.C.

AASHTO (2002) The Bottom Line Report. Retrieved April 15, 2203, from

http://www.transportation.org/bottomline/bottomline202.pdf

Bureau of Transportation Statistics (BTS); Highway Statistics Publications (1991-2001).

Counties Annual Operational Reports, 1998 and 2000

Cambridge Systematics, Inc. Personal communications, 2004, regarding HERS Improvement Codes

Table and Improvement Cost Tables.

Davis, S. C., and Diegel, S. W., (2003) Transportation Energy Data Book: Edition 23, Oak Ridge National Laboratory, ORNL-6970

Dornan, D.L. (2000) Asset Management and Innovative Finance. Conference Proceedings 24, pp.145-156, Second National Conference on transportation Finance, Transportation Research Board.

Drike, K. (2001). An Evaluation of Innovative Financing Techniques for Indiana. M.S. Thesis, Purdue University, West Lafayette.

Drike, K., Genetti, A. V., Sinha, K. C. (2002). An Evaluation of Innovative Financing Techniques for Indiana (No. FHWA/IN/JTRP-2002/11). Joint Transportation Research Program. Purdue University, West Lafayette, IN.

Elridge, J. (September 2002) Modeling the Cost of Bridge Replacement for Indiana County Highway Department; Purdue University, West Lafayette IN. 
FHWA (1987), Highway Performance Monitoring System: Field Manual for the Continuing Analytical and Statistical Database, Appendix J, Order M5600.1A, OMB No. 2125-0028

FHWA (2000). Highway Statistics 1993-1999. Washington, D.C.: US Department of Transportation.

FHWA (2001a). Higbway Statistics 2001, Table VM-2, Annual Vehicle-Miles, October 2002. Retrieved May 28, 2003, from http://www.fhwa.dot.gov/ohim/

FHWA (2001b). Highway Statistics 2001, Table HM-60, Lane-Miles, October 2002. Retrieved May 28, 2003, from http://www.fhwa.dot.gov/ohim/

FHWA (2001c). Highway Economic Requirements System for State Use: Office of Asset Management.

FHWA (2001d). HERS/ST Overview Report (Draft).

FHWA (2002). HERS-ST Highway Economic Requirements System-State Version Pilot Program Report 2002.

FHWA (2004a). Personal email communication from Bryant Gross of FHWA, 2004, regarding Construction Price Index 1960-2002.

FHWA (2004b). Personal email communication from David Winter of FHWA Office of Asset Management, February 2004, regarding HPMS 2002Data.

GASB. (1999). GASB Statement No. 34: Basic Financial Statements and Management's Discussion and Analysis for State and Local Governments. Governmental Accounting Standards Board, Norwalk, CT.

Geoffroy, D. N. (1996). Synthesis of Highway Practice 223: Cost-effective Preventive Pavement Maintenance. Washington, D.C: Transportation Research Board, National Research Council.

Giglio, J. M., Williams, J. (2000) Strategic Alternatives for Financing the Higbway System. Conference Proceedings 24, pp. 199-203, Second Annual Conference on Transportation Finance.

Gion, L. C., Gough, J., Vitale, J. D., Sinha, K. C., \& Woods, R. E. (1993). The Development of Optimal Strategies for Maintenance, Rehabilitation and Replacement of Highway Bridges, User's Manual for the Implementation of the Indiana Bridge Management System. Joint Highway Research Project, Indiana. 
Goldman, T., Corbett, S., Wachs, M. (2001). Local Option Transportation Taxes in the United States. Institute of Transportation Studies, University of California, Berkeley.

Gulen, S., Woods, R., Weaver, J., and Anderson, V.L.,. (1994). Correlation of Pavement Serviceability Rating with International Roughness Index. Transportation Research Record 1435.

Hartgen, D. T. (2000). North Carolina Highways: Performance, Needs and Funding Options. John Locke Foundation.

Indiana Legislative Services Agency, (2003). Indiana Handbook of Taxes, Revenues, and Appropriations. Indianapolis. IN.

INDOT (2002) Highway Performance Monitoring System (HPMS) data Base, Indianapolis, Indiana

INDOT (1994). Indiana Design Manual Part V Road Design. In (Vol. I, pp. 40-41(41) - 40-41(47)).

INDOT (2000). 2000 Pavement Surface Report, December, 2000. INDOT Pavement Management Section, Program Development Division.

INDOT (2003a). 2003 Annual Report, FY 2002 Transportation Revenue Analysis.

INDOT (2003b). 2003 Annual Report, Road Mileage and Control.

INDOT (2003c). INDOT 2003 Quick Facts, Budget and Fiscal Management Division of INDOT.

INDOT (2003d). VMT by Jurisdiction 1994-2002. Personal communication. HPMS Submittal Data for the 1990 Adjusted Urban Area Boundaries provided by Leah Snow of the Program Development Division of INDOT.

INDOT (2003e). I-69: Evansville to Indianapolis Tier 1 Draft Environmental Impact Statement, Summary Chapter: INDOT.

INDOT (2003f). FY-2004 Pavement Preservation Guidance (Draft) (April 10, 2003 Inter-Office Communication): INDOT Program Development Division. 
Lamptey, G. (2004). Development of Analytical and Software Tools for Highway Safety Management. M.S.

Thesis, Purdue University, West Lafayette.

Local Technical Assistance Program (LTAP), (2000a) Summary of Local Road and Street Inventory Data for Indiana LPA's.

Local Technical Assistance Program (LTAP), (2000b) Bridge Sufficiency Ratings Reports, Purdue University, Indiana

Local Technical Assistance Program (LTAP), (2002) Summary of Highway Revenues, Distributions \& Expenses for Indiana Counties, Cities \& Towns (RP-4-2002)

Local Technical Assistance Program (LTAP), (2002) Guide to Revenue Calculations: Local Option Highway User Tax (RP-5-2002).

Local Technical Assistance Program (LTAP), (May 2001), Need Assessment for Local Roads and Streets (SP-1a-2001).

Local Technical Assistance Program (LTAP), (2001) Road Condition Survey of 3,200 miles of county roads in eight separate counties, Fall 200-Spring 2001, Purdue University, Indiana.

Local Technical Assistance Program (LTAP), (2003) Pavement reconstruction costs per square yard from Local Highway Agencies (Monroe County, Dubois County, and Hamilton County).

Metcalfe, J.A. (1969). Financing the Highway Needs of Indiana. M.S. Thesis, Purdue University, West Lafayette.

Morlok, E. K. (1978). Introduction to Transportation Engineering and Planning. McGraw-Hill.

Morris, H., DeCicco, John. (1997). Extent to Which User Fees Cover Road Expenditures in the United States. Transportation Research Record, 1576, 56-62.

Palmer, J. (2003). Intergovernmental Issues in Indiana: 2002 IACIR Survey. Indianapolis: Center for Urban Policy and the Environment. Indiana University - Purdue University- Indianapolis School of Public and Environmental Affairs. 
Pennington, A. (1995). Project Pioneers Fund Global Infrastructure. Site Selection, 1-3.

Puentes, R., Prince, Ryan. (2003). Fueling Transportation Finance: A Primer on the Gas Tax. The Brookings Institution Series on Transportation Reform, 1-19.

Reed, C. (2002, January 12, 2002). Balanced budget news from the Office of Governor Frank O'Bannon. The Financial Times of Indiana, p. 1, Indianapolis

Riverson, J. (1985); Unpaved road maintenance management in local highway systems; PhD Dissertation; Purdue University, West Lafayette IN.

Rodriguez, M. M. (2004). An Assessment of Preservation Needs of State Highway Bridges in Indiana. M.S. Thesis, Purdue University, West Lafayette, IN.

Shahin, M. Y. (1994). Pavement Management for Airports, Roads and Parking Lots. New York: Chapman \& Hall.

STPP (2003). Surface Transportation Policy Project State Scorecard Insert. Progress, XIII, 1-11, Washington D.C.

U.S. Department of Commerce (2002), Bureau of Economic Analysis, Survey of Current Business, Washington D.C.

USDOT (2003). Economic Analysis Primer: United States Department of Transportation, Federal Highway Administration, Office of Asset Management, Washington D.C.

Varma, A., and Sinha, K.C. (1990). On User Charges for Highway Financing, Transportation Research: A, Volume 24A. No. 4, pp. 293-302.

Varma, A., and Sinha, K.C., and Spalding, J.L., (1991). The Development of A Highway Revenue Forecasting Model for Indiana, Joint Highway Research Project, FHWA/IN/JHRP-91/5.

Vitale, J. D. (1997). The Development of Optimal Policies in the Programming of Bridge Rehabilitation and Replacement Activities for Indiana. M.S. Thesis, Purdue University, West Lafayette. 
INDOT (2003g). Indiana Transportation Funding Flowchart for Fiscal Year 2003., INDOT Budget and Fiscal Management Division.

INDOT (2004a). 2004 Annual Report. Retrieved September 26, 2004, from www.in.gov/dot/div/communications

INDOT (2004b). I-69 Projects in the Long Range Plan. Personal Communication with Jay Mitchell of the Program Development Division of INDOT.

INDOT (2004c). INDOT 2000-2025 Long Range Plan Projects Summary, Program Development Division of INDOT.

Indiana University (2002), Indiana Factbook 2002, School of Business, Indiana Business Research Center, Indianapolis

ITE (1999). Traffic Engineering Handbook (5th Ed.). Washington, D.C.

Jackson, A. (2003). Motor Vehicle Highway Fund Historic Data. Data obtained through personal communication with A. Jackson of INDOT Budget and Fiscal Management Division, April 7, 2004.

Jackson, A. (2004a). Personal communication with A. Jackson of INDOT Budget and Fiscal Management Division, August 5, 2004.

Jackson, A. (2004b). Historical INDOT Financial Plan Data. Personal communication with A. Jackson of INDOT Budget and Fiscal Management Division, January, 2004. Indianapolis.

Labi, S., and Sinha, K. C. (2003). The Effectiveness of Maintenance and Its Impact on Capital Expenditures (No. FHWA/IN/JTRP-2002/27). Joint Transportation Research Program, Purdue University. West Lafayette, IN.

Lamptey, G., Ahmad, M., Labi, S., and Sinha, K.C. (2004). Life Cycle Cost Analysis for Pavement Design Procedures. (No. FHWA/IN/JTRP-2004/28). Draft final report. Joint Transportation Research Program, Purdue University, West Lafayette, IN. 
Wachs, M. (2003). Local Option Transportation Taxes. Devolution as Revolution. Access, 22 (Spring), 9-15.

Woods \& Poole, E. (2002). The Complete Economic and Demographic Data Source: CEDDS, 2003. 
APPENDICES 


\section{Appendix A. INDOT Contracts Unit Pavement Treatment Costs}

Table A.1 Unit Pavement Treatment Costs

\begin{tabular}{|c|c|c|c|c|c|c|}
\hline \multirow{2}{*}{ Treatment } & \multirow{2}{*}{$\begin{array}{c}\text { Pavement } \\
\text { Type }\end{array}$} & \multirow{2}{*}{$\begin{array}{l}\text { No.of } \\
\text { Obs. }\end{array}$} & \multicolumn{4}{|c|}{$\begin{array}{l}\text { Cost per Lane Mile } \\
(1000 \text { s }) 2002 \$\end{array}$} \\
\hline & & & Mean & Min. & Max. & $\begin{array}{l}\text { Std. } \\
\text { Dev. }\end{array}$ \\
\hline Added Travel Lanes & COMP & 1 & 21798 & 21798 & 21798 & 0 \\
\hline Added Travel Lanes & HMA & 46 & 2111 & 150 & 15170 & 2541 \\
\hline Added Travel Lanes & PCC & 14 & 2445 & 236 & 7103 & 2273 \\
\hline Added Travel Lanes, Bituminous & HMA & 1 & 263 & 263 & 263 & 0 \\
\hline Added Travel Lanes, Concrete & PCC & 6 & 4628 & 486 & 16027 & 5689 \\
\hline Asphalt Patching & HMA & 1 & 38 & 38 & 38 & 0 \\
\hline Auxillary Lane Construction & HMA & 3 & 149 & 9 & 414 & 229 \\
\hline Auxillary Lanes, Acel \& Dcel & HMA & 1 & 726 & 726 & 726 & 0 \\
\hline Bridge Deck Overlay & HMA & 1 & 295 & 295 & 295 & 0 \\
\hline Bridge Rehabilitation Or Repair & PCC & 0 & 0 & 0 & 0 & 0 \\
\hline Bridge Removal & HMA & 1 & 1067 & 1067 & 1067 & 0 \\
\hline Crack \& Seat Composite Pavement \& HMA Overlay & COMP & 20 & 673 & 230 & 3210 & 735 \\
\hline Crack \& Seat PCCP \& HMA Overlay & PCC & 5 & 209 & 2 & 379 & 188 \\
\hline Crack \& Seat PCCP \& HMA Overlay (P3R) & PCC & 6 & 644 & 2 & 3356 & 1332 \\
\hline Diamond Grinding & PCC & 0 & 0 & 0 & 0 & 0 \\
\hline Ditch Relocation & HMA & 1 & 807 & 807 & 807 & 0 \\
\hline Drainage Ditch Correction & HMA & 2 & 534 & 234 & 834 & 424 \\
\hline Dual Lane Existing Route & PCC & 2 & 2065 & 1430 & 2699 & 897 \\
\hline Full And Shallow Depth Patching & HMA & 1 & 31 & 31 & 31 & 0 \\
\hline Full And Shallow Depth Patching & PCC & 1 & 298 & 298 & 298 & 0 \\
\hline HMA Overlay, Functional & COMP & 17 & 314 & 7 & 1652 & 454 \\
\hline HMA Overlay, Functional & HMA & 88 & 210 & 3 & 3086 & 469 \\
\hline HMA Overlay, Functional & PCC & 4 & 129 & 9 & 406 & 187 \\
\hline HMA Overlay, Preventive Maintenance & COMP & 1 & 37 & 37 & 37 & 0 \\
\hline HMA Overlay, Preventive Maintenance & HMA & 26 & 73 & 18 & 296 & 71 \\
\hline HMA Overlay, Structural & HMA & 18 & 236 & 60 & 1022 & 249 \\
\hline Install New Guard Rail & HMA & 1 & 199 & 199 & 199 & 0 \\
\hline Install New Small Structure & HMA & 1 & 80 & 80 & 80 & 0 \\
\hline Interchange Modification & COMP & 1 & 1156 & 1156 & 1156 & 0 \\
\hline Interchange Modification & PCC & 0 & 0 & 0 & 0 & 0 \\
\hline Intersection Improvement & HMA & 5 & 234 & 190 & 301 & 45 \\
\hline Intersection Improvement & PCC & 1 & 123 & 123 & 123 & 0 \\
\hline Mill Full Depth And Bit Overlay & COMP & 3 & 356 & 17 & 753 & 372 \\
\hline Mill Full Depth And Bit Overlay & HMA & 2 & 366 & 226 & 507 & 199 \\
\hline New Br, Cont.Pres.Conc.Box Beam & HMA & 1 & 138 & 138 & 138 & 0 \\
\hline New Road Construction - CRC & PCC & 5 & 2991 & 1244 & 5875 & 1934 \\
\hline
\end{tabular}


Table A.1 Unit Costs of Pavement Treatments (Continued)

\begin{tabular}{|c|c|c|c|c|c|c|}
\hline \multirow{2}{*}{ Treatment } & \multirow{2}{*}{$\begin{array}{c}\text { Pavement } \\
\text { Type }\end{array}$} & \multirow{2}{*}{$\begin{array}{c}\text { No.of } \\
\text { Obs. }\end{array}$} & \multicolumn{4}{|c|}{$\begin{array}{l}\text { Cost per Lane Mile } \\
\text { (1000s) 2002\$ }\end{array}$} \\
\hline & & & Mean & Min. & Max. & $\begin{array}{l}\text { Std. } \\
\text { Dev. }\end{array}$ \\
\hline New Road Construction, Composite & COMP & 1 & 2966 & 2966 & 2966 & 0 \\
\hline New Road Construction, HMA & HMA & 32 & 2545 & 14 & 22561 & 4472 \\
\hline New Road Construction, PCC & PCC & 31 & 3620 & 368 & 11010 & 2988 \\
\hline New Road, HMA Paving Only & HMA & 2 & 1424 & 720 & 2127 & 995 \\
\hline New Road, PCC Paving Only & PCC & 3 & 1224 & 484 & 2238 & 909 \\
\hline New Sign Installation & PCC & 1 & 2760 & 2760 & 2760 & 0 \\
\hline Other Methods Of Rehabing Pavement & COMP & 1 & 275 & 275 & 275 & 0 \\
\hline Other Methods Of Rehabing Pavement & HMA & 1 & 272 & 272 & 272 & 0 \\
\hline Other Type Project (Miscellaneous) & HMA & 1 & 994 & 994 & 994 & 0 \\
\hline Patch and Rehab HMA Pavement & COMP & 1 & 8226 & 8226 & 8226 & 0 \\
\hline Patch and Rehab HMA Pavement & HMA & 3 & 57 & 42 & 65 & 13 \\
\hline Patch and Rehab PCC Pavement & PCC & 2 & 97 & 27 & 167 & 100 \\
\hline Pavement Repair or Rehabilitation & COMP & 2 & 723 & 181 & 1265 & 766 \\
\hline Pavement Repair or Rehabilitation & HMA & 43 & 311 & 14 & 2718 & 505 \\
\hline Pavement Repair or Rehabilitation & PCC & 1 & 7 & 7 & 7 & 0 \\
\hline Pavement Replacement, HMA & HMA & 3 & 956 & 23 & 2268 & 1170 \\
\hline Pavement Replacement, New PCC & COMP & 1 & 3918 & 3918 & 3918 & 0 \\
\hline Pavement Replacement, New PCC & HMA & 5 & 1394 & 311 & 2376 & 917 \\
\hline Pavement Replacement, New PCC & PCC & 7 & 1454 & 441 & 2597 & 754 \\
\hline PCCP Cleaning and Sealing Joints & PCC & 10 & 269 & 16 & 1828 & 553 \\
\hline PCCP on Asphalt Pavement & HMA & 12 & 89 & 15 & 372 & 98 \\
\hline PCCP on PCC Pavement & PCC & 2 & 1247 & 509 & 1985 & 1044 \\
\hline PCCP Patching & PCC & 15 & 1145 & 20 & 10662 & 2717 \\
\hline Repair PCCP \& HMA Overlay & PCC & 7 & 156 & 10 & 561 & 217 \\
\hline Resurface (Partial 3-R Standards) & COMP & 18 & 383 & 47 & 1960 & 489 \\
\hline Resurface (Partial 3-R Standards) & HMA & 1405 & 297 & 8 & 7282 & 585 \\
\hline Resurface (Partial 3-R Standards) & PCC & 11 & 826 & 52 & 3503 & 1110 \\
\hline Resurface over Asphalt Pavement (Partial 3-R Standards) & HMA & 18 & 361 & 34 & 2619 & 719 \\
\hline Resurface PCC Pavement (Partial 3-R Standards) & PCC & 2 & 136 & 134 & 139 & 3 \\
\hline Road Reconstruction (3R/4R Standards) & COMP & 56 & 673 & 39 & 3394 & 749 \\
\hline Road Reconstruction (3R/4R Standards) & HMA & 104 & 784 & 39 & 4378 & 956 \\
\hline Road Reconstruction (3R/4R Standards) & PCC & 18 & 2118 & 128 & 9686 & 2749 \\
\hline Rubblize PCCP \& HMA Overlay & PCC & 18 & 2322 & 131 & 10789 & 2728 \\
\hline Sight Distance Improvement & HMA & 1 & 505 & 505 & 505 & 0 \\
\hline Small Structure, Replacement & HMA & 2 & 447 & 171 & 723 & 391 \\
\hline Surface Treatment, PM & HMA & 10 & 62 & 8 & 382 & 114 \\
\hline Vertical Sight Distance Correction & HMA & 1 & 906 & 906 & 906 & 0 \\
\hline Wedge And Level Only & HMA & 65 & 53 & 2 & 424 & 83 \\
\hline Widen Pavement and HMA Overlay & HMA & 6 & 262 & 25 & 648 & 230 \\
\hline
\end{tabular}


Appendix B. HERS Treatment Costs

Table B.1 HERS-IN Indiana-specific Costs

\begin{tabular}{|c|c|c|c|c|c|c|c|c|}
\hline & \multirow[b]{3}{*}{ Terrain } & \multicolumn{7}{|c|}{$\begin{array}{c}\text { Improvement Costs per Lane Mile } \\
\text { (Thousands of } 1997 \text { Dollars) }^{1}\end{array}$} \\
\hline & & \multicolumn{4}{|c|}{ Resurface } & \multicolumn{3}{|c|}{ Reconstruct } \\
\hline & & & $\begin{array}{l}\text { Improve } \\
\text { Shoulders }\end{array}$ & $\begin{array}{l}\text { Widen } \\
\text { Lanes }\end{array}$ & $\begin{array}{c}\text { Add } \\
\text { Lanes }\end{array}$ & & $\begin{array}{l}\text { Widen } \\
\text { Lanes }\end{array}$ & $\begin{array}{c}\text { Add } \\
\text { Lanes }\end{array}$ \\
\hline Rural & & & & & & & & \\
\hline Interstate & $\begin{array}{l}\text { Flat } \\
\text { Rolling }\end{array}$ & $\begin{array}{l}90 \\
94\end{array}$ & $\begin{array}{l}187^{2} \\
204\end{array}$ & $\begin{array}{l}396 \\
429\end{array}$ & $\begin{array}{l}534 \\
578\end{array}$ & $\begin{array}{c}567^{2} \\
623\end{array}$ & $\begin{array}{l}644 \\
702\end{array}$ & $\begin{array}{c}976 \\
1064\end{array}$ \\
\hline $\begin{array}{l}\text { Other } \\
\text { Principal } \\
\text { Arterial }\end{array}$ & $\begin{array}{l}\text { Flat } \\
\text { Rolling }\end{array}$ & $\begin{array}{l}64 \\
68\end{array}$ & $\begin{array}{l}135 \\
147\end{array}$ & $\begin{array}{l}285 \\
309\end{array}$ & $\begin{array}{l}385 \\
417\end{array}$ & $\begin{array}{l}408 \\
449\end{array}$ & $\begin{array}{l}464 \\
505\end{array}$ & $\begin{array}{l}703 \\
766\end{array}$ \\
\hline $\begin{array}{l}\text { Minor } \\
\text { Arterial }\end{array}$ & $\begin{array}{l}\text { Flat } \\
\text { Rolling }\end{array}$ & $\begin{array}{l}58 \\
62\end{array}$ & $\begin{array}{l}121 \\
132\end{array}$ & $\begin{array}{l}255 \\
279\end{array}$ & $\begin{array}{l}346 \\
376\end{array}$ & $\begin{array}{l}368 \\
405\end{array}$ & $\begin{array}{l}417 \\
455\end{array}$ & $\begin{array}{l}633 \\
690\end{array}$ \\
\hline $\begin{array}{l}\text { Major } \\
\text { Collector }\end{array}$ & $\begin{array}{l}\text { Flat } \\
\text { Rolling }\end{array}$ & $\begin{array}{l}52 \\
55\end{array}$ & $\begin{array}{l}111 \\
119\end{array}$ & $\begin{array}{l}231 \\
251\end{array}$ & $\begin{array}{l}313 \\
339\end{array}$ & $\begin{array}{l}331 \\
365\end{array}$ & $\begin{array}{l}377 \\
411\end{array}$ & $\begin{array}{l}571 \\
623\end{array}$ \\
\hline Urban & & & & & & & & \\
\hline $\begin{array}{l}\text { Freeway/ } \\
\text { Expressway }\end{array}$ & & 121 & $275^{2}$ & 588 & $1762^{3}$ & $755^{2}$ & 1081 & 1472 \\
\hline $\begin{array}{l}\text { Other } \\
\text { Divided }\end{array}$ & & 88 & 201 & 423 & $1104^{3}$ & 546 & 779 & 1060 \\
\hline Undivided & & 79 & 181 & 381 & $824^{2,3}$ & 492 & 701 & 954 \\
\hline
\end{tabular}

Source: Cambridge Systematics, Inc., Major Corridor Investment Benefit Analysis System: Model

Documentation, Part IV: HERS/IN Users Manual, Sept. 1998, Table 2.7.

1. Excludes costs of alignment improvements.

2. Derived directly from INDOT costs.

3. These costs are per added lane-mile; all other costs are per lane-mile. 
Appendix C. AAMEX Model Curves

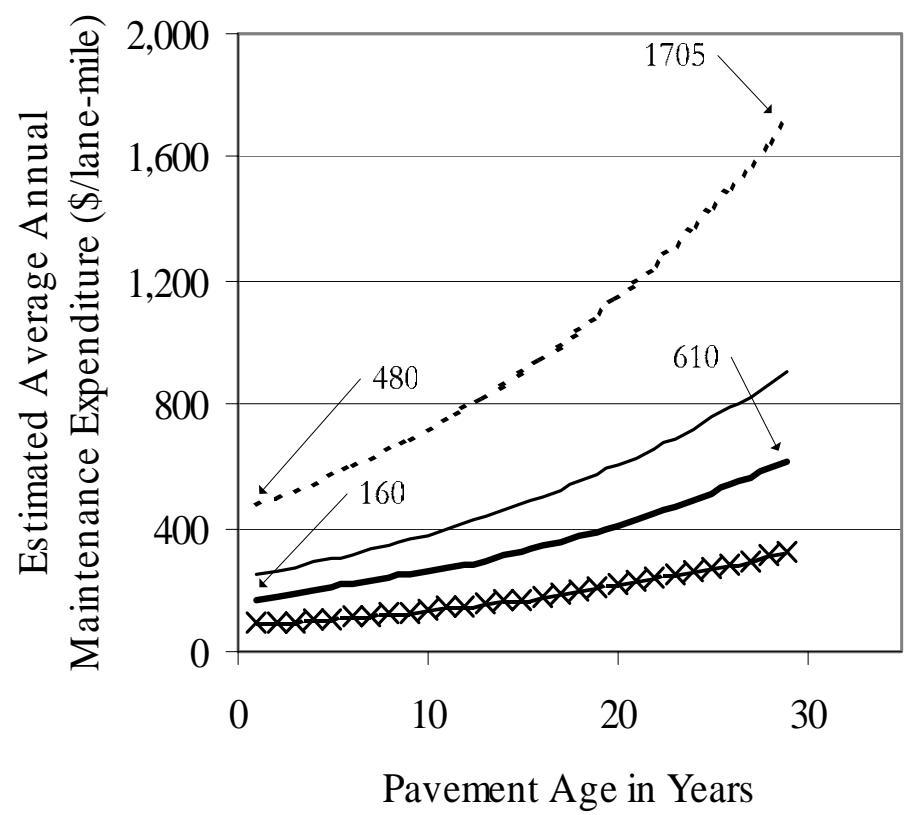

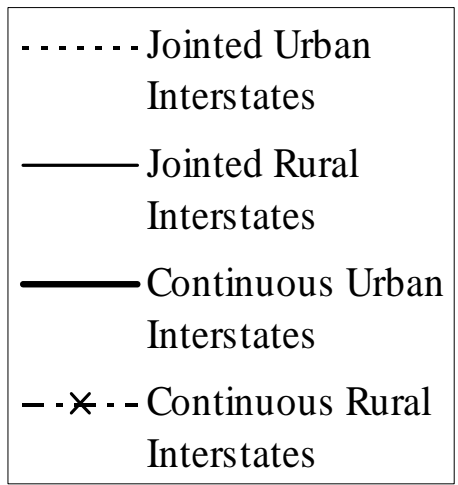

Figure C.1 Fitted Values for AAMEX Model, Rigid Interstate Pavements 1995\$

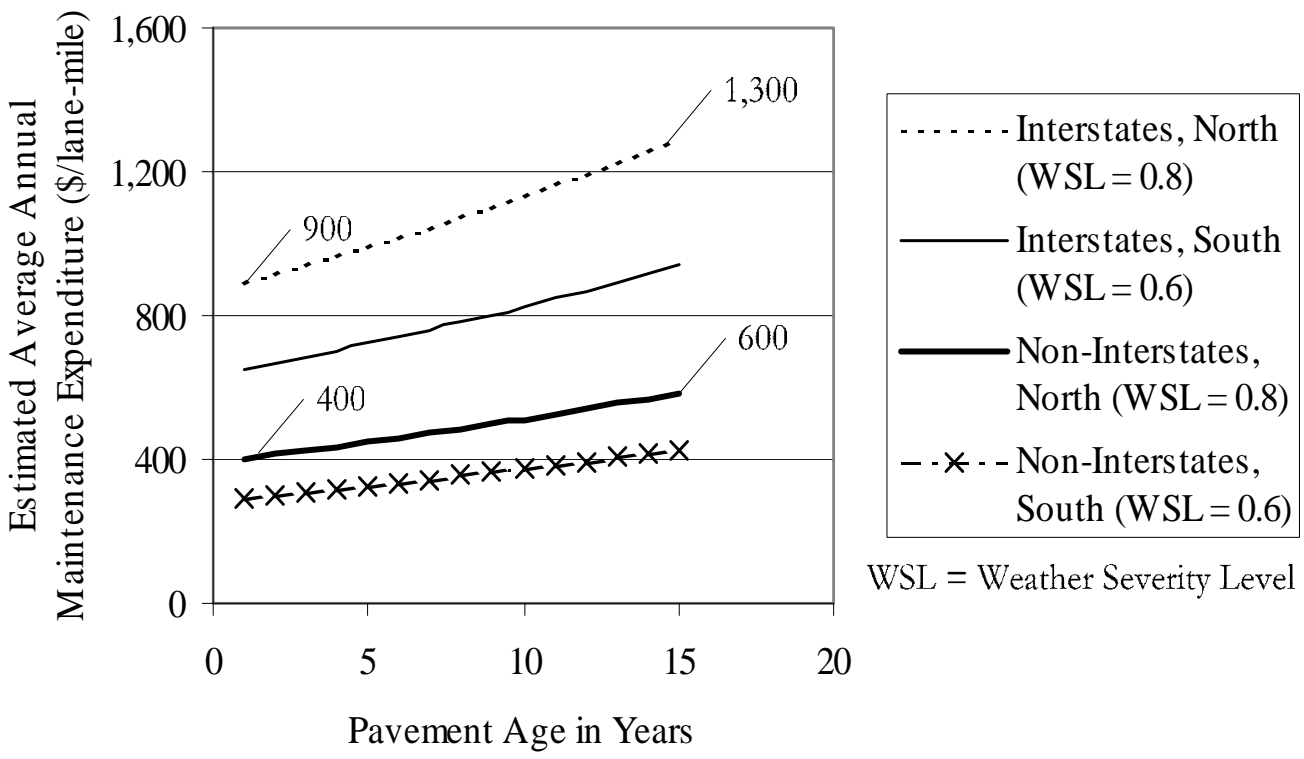

Figure C.2 Fitted Values for AAMEX Model, Rigid Interstate Pavements 1995\$ 

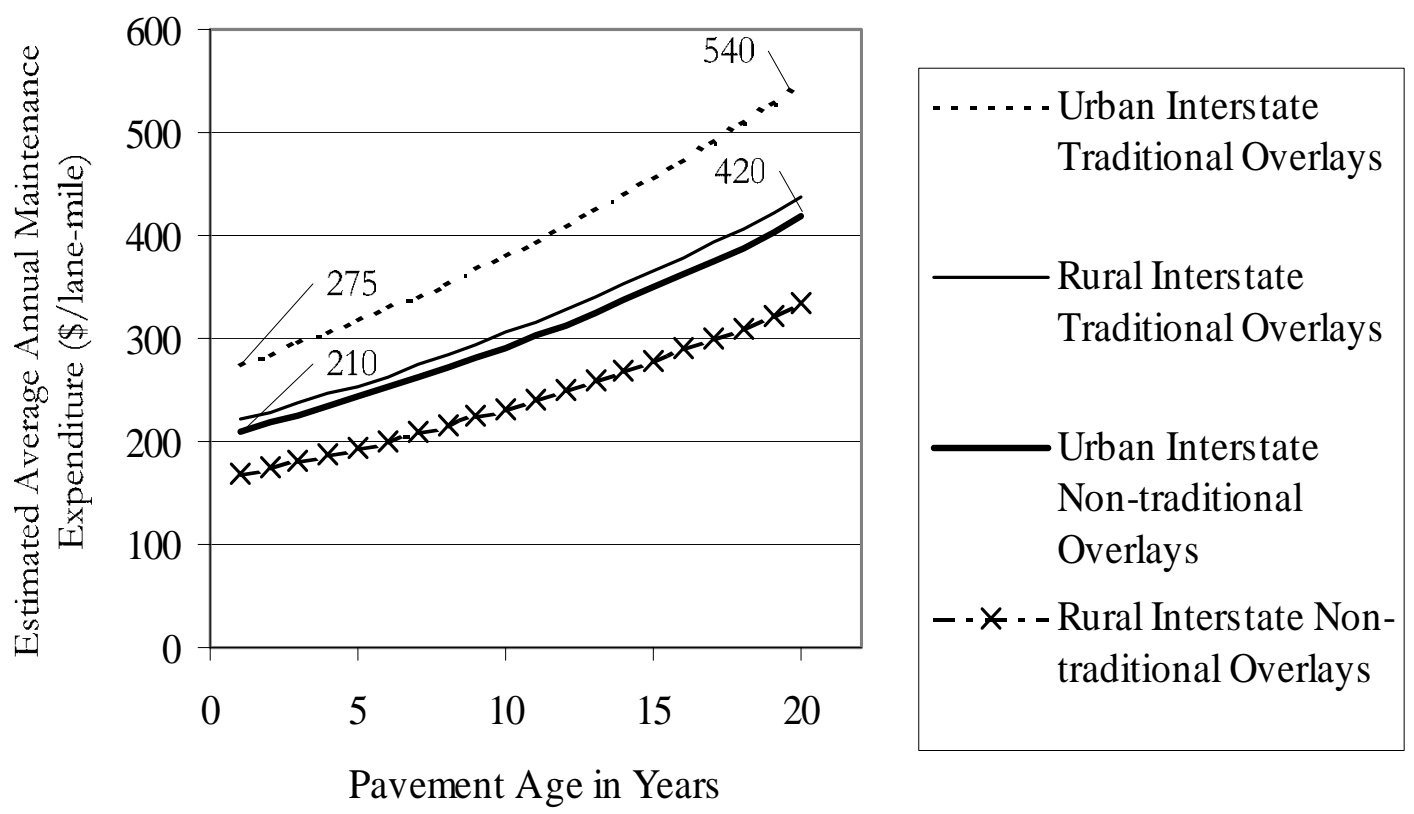

Figure C.3 Fitted Values for AAMEX Model, Interstate COMP Pavements 1995\$
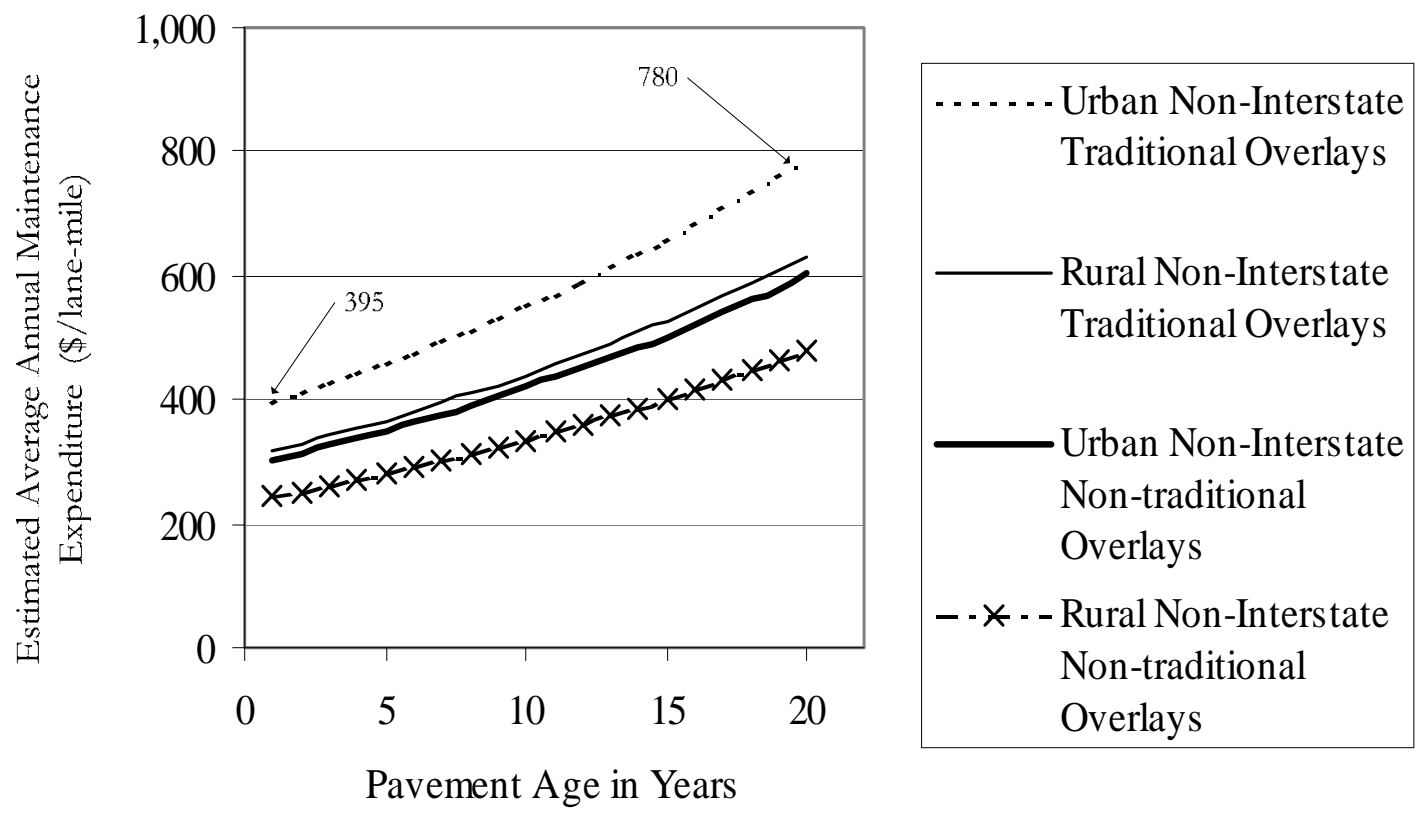

Figure C.4 Fitted Values for AAMEX Model, Non-Interstate COMP Pavements 1995\$ 
Appendix D. HERS-ST Routine Maintenance Costs

Table D.1 Maintenance Costs for Flexible Pavements (\$ Year 1984)

\begin{tabular}{|c|c|c|}
\hline Final PSI & $\begin{array}{c}\text { Maintenance Cost } \\
\text { Between PSI Levels } \\
\text { (\$/lane mile) }\end{array}$ & $\begin{array}{l}\text { Cumulative Cost } \\
\text { (\$/lane mile) }\end{array}$ \\
\hline $\begin{array}{r}\text { Low SN/traffic: }(\mathrm{SN}=2.16) \\
4.0 \\
3.5 \\
3.0 \\
2.5 \\
2.0 \\
1.5\end{array}$ & $\begin{array}{r}221.57 \\
767.03 \\
1314.95 \\
1859.47 \\
2413.74 \\
2957.34\end{array}$ & $\begin{array}{r}221.57 \\
988.60 \\
2302.55 \\
4163.02 \\
6576.76 \\
9534.10\end{array}$ \\
\hline $\begin{array}{c}\text { Medium SN/traffic: }(\mathrm{SN}= \\
3.60) \\
4.0 \\
3.5 \\
3.0 \\
2.5 \\
2.0 \\
1.5\end{array}$ & $\begin{array}{r}339.10 \\
1174.05 \\
2012.72 \\
2845.76 \\
3604.98 \\
4526.45\end{array}$ & $\begin{array}{r}339.10 \\
1513.15 \\
3525.87 \\
6371.63 \\
10066.61 \\
14593.06\end{array}$ \\
\hline $\begin{array}{r}\text { High SN/traffic: }(\mathrm{SN}=5.04) \\
4.0 \\
3.5 \\
3.0 \\
2.5 \\
2.0 \\
1.5\end{array}$ & $\begin{array}{r}456.63 \\
1518.05 \\
2710.50 \\
3832.04 \\
4976.21 \\
6095.55\end{array}$ & $\begin{array}{r}456.63 \\
2037.38 \\
4748.18 \\
8580.22 \\
13556.43 \\
19651.98\end{array}$ \\
\hline
\end{tabular}

Source: Matthew W. Witczak and Gonzalo R. Rada, Microcomputer Solution of the Project Level PMS Life Cycle Cost Model, University of Maryland, Department of Civil Engineering, prepared for Maryland Department of Transportation, State Highway Administration, Baltimore, MD., December 1984, p. 132. 
Appendix E. HPMS Record Description

Table E.1 HPMS Record Layout

\begin{tabular}{|c|c|c|}
\hline Item No. & Variable Name & Description \\
\hline 1 & YR & Year \\
\hline 2 & STATE & State code \\
\hline 3 & UNITS & Reporting units (English or metric) \\
\hline 5 & SECTIONID & Section identification \\
\hline 6 & CNTY & County code \\
\hline 8 & $\mathrm{SCF}^{1}$ & State control field \\
\hline 10 & LRSID $^{1}$ & LRS identification \\
\hline 11 & BEGMP $^{1}$ & LRS beginning point \\
\hline 12 & ENDMP $^{1}$ & LRS ending point \\
\hline 13 & RURURB & Rural/Urban designation \\
\hline 17 & FC & Functional system \\
\hline 18 & GFC & Generated functional system code \\
\hline 20 & $\mathrm{UNBLT}^{2}$ & Unbuilt facility code \\
\hline 27 & FT & Type of facility (one way or two way) \\
\hline 30 & SLEN & Section length \\
\hline 33 & AADT & Annual average daily traffic \\
\hline 34 & LANES & Number of through lanes \\
\hline 35 & IRICOD $^{3}$ & International Roughness Index \\
\hline 36 & PSR $^{3}$ & Pavement condition \\
\hline 37 & $\mathrm{HOV}$ & HOV operations \\
\hline 47 & SECNUM & HPMS sample identifier or other section identifier \\
\hline 49 & EXPFAC & Expansion factor for standard HPMS sample \\
\hline 50 & SURF & Surface type \\
\hline 51 & SNORD & $\mathrm{SN}$ or $\mathrm{D}$ \\
\hline 52 & CLIMATE & Climate zone \\
\hline 53 & IMPYR & Year of surface improvement \\
\hline 54 & LANEW & Lane width \\
\hline 55 & ACCESS & Access control \\
\hline 56 & MEDT & Median type \\
\hline 57 & MEDW & Median width \\
\hline 58 & SHLDT & Shoulder type \\
\hline
\end{tabular}

1.Variable copied to output files but not otherwise used by HERS-ST.

2.Not used by HERS-ST 2.0.

3.HERS-ST requires either IRI or PSR. If both are provided, the PSR/IRI indicator (on Line 33 of RUNSPEC) identifies the value to be used.

4.Optional input. Will be calculated by HERS-ST if not coded. 
Table E.2 HPMS Record Layout (continued)

\begin{tabular}{|c|c|c|}
\hline Item No. & Variable Name & Description \\
\hline 59 & RSHLDW & Right shoulder width \\
\hline 60 & LSHLDW & Left shoulder width \\
\hline 61 & PKPARK & Peak parking \\
\hline 62 & WDFEAS & Widening feasibility \\
\hline $63-68$ & LCURVE(I) & Curves by class \\
\hline 69 & HORALN & Horizontal alignment adequacy \\
\hline 70 & TERRN & Type of terrain \\
\hline 71 & VERALN & Vertical alignment adequacy \\
\hline $72-77$ & LGRADE(I) & Grades by class \\
\hline 78 & PSD & Percent passing-sight distance \\
\hline 79 & WDS $^{4}$ & Weighted design speed \\
\hline 80 & SPDLIM & Posted speed limit \\
\hline 81 & PCPKSU & Percent peak single-unit commercial vehicles \\
\hline 82 & PCAVSU & Percent average daily single-unit commercial vehicles \\
\hline 83 & PCPKCM & Percent peak combination commercial vehicles \\
\hline 84 & PCAVCM & Percent average daily combination commercial vehicles \\
\hline 85 & KFAC & K factor \\
\hline 86 & DFAC & Directional factor \\
\hline 87 & PLANES & Number of peak lanes in peak direction \\
\hline 88 & LTURN & Turning lanes - left \\
\hline 89 & RTURN & Turning lanes - right \\
\hline 91 & PCTGRN & Percent green time \\
\hline 92 & NSIG & Number of intersections with traffic signals \\
\hline 93 & NSTOP & Number of intersections with stop signs on sample section \\
\hline 94 & NOINTS & Number of other intersections \\
\hline 95 & $\mathrm{CAPAC}^{4}$ & Peak capacity (peak direction) \\
\hline 97 & FAADT & AADT in future year (FYEAR) \\
\hline 98 & FADTYR & Future year for AADT forecast \\
\hline
\end{tabular}

1 Variable copied to output files but not otherwise used by HERS-ST.

2 Not used by HERS-ST 2.0.

3 HERS-ST requires either IRI or PSR. If both are provided, the PSR/IRI indicator (on Line 33 of RUNSPEC) identifies the value to be used.

4 Optional input. Data will be calculated by HERS-ST if not coded. 
Appendix F. HERS-ST Default Values

Table F.1 Pavement Thickness After Improvement (in inches)

\begin{tabular}{|lcc|}
\hline Forecast ESALs over Design Life & \multicolumn{2}{c|}{ Pavement Type } \\
& Rigid $^{\mathrm{d}}$ & Flexible $^{\mathrm{b}} \mathrm{c}$ \\
\hline$\leq 50,000$ & 1.5 & 6.5 \\
$50,001-150,000$ & 2.5 & 6.5 \\
$150,001-500,000$ & 3.0 & 6.5 \\
$500,001-2,000,000$ & 4.0 & 8.0 \\
$2,000,001-7,000,000$ & 5.0 & 9.5 \\
$>7,000,000$ & 5.5 & 10.5 \\
\hline
\end{tabular}

HERS-ST Technical Report, Table 8-6, page 8-5.

a. American Association of State Highway and Transportation Officials, AASHTO Guide for Design of Pavement Structures, Washington, D.C., 1986.

b. Thicknesses shown for flexible pavements are also used for resurfacing flexible pavements with a flexible overlay.

c. For low-type pavement, assume a surface treatment only.

d. E.J. Yoder and M.W. Witczak, Principles of Pavement Design, John Wiley, New York City, 1975 
Appendix G. Bridge Data Items

Table G.1 IBMS Data Items

\begin{tabular}{|c|c|c|}
\hline DATA TYPE & DATA ITEM & VARIABLE \\
\hline \multirow{2}{*}{ Identification } & Bridge-Number & BRGN \\
\hline & Contract-Num & CONT NU \\
\hline \multirow{5}{*}{ Location } & Road-Num-Over & RD NB \\
\hline & County-Code & CNTY CD \\
\hline & District-Code & DST CD \\
\hline & Latitude & LAT \\
\hline & Longitude & LONG \\
\hline \multirow{7}{*}{$\begin{array}{c}\text { Operational } \\
\text { Characteristics }\end{array}$} & Features-Intersected & FEA INT \\
\hline & Facility-Carried & FAC CAR \\
\hline & Func-Class-Over & Fco \\
\hline & Hway-Sys-O & HWY SYS \\
\hline & Adt-Over & ADT \\
\hline & Lanes-Over & $\mathrm{N}$ \\
\hline & Detour-Length & DL \\
\hline \multirow{11}{*}{$\begin{array}{l}\text { Bridge Physical } \\
\text { Characteristics }\end{array}$} & Deck-Width & TDW \\
\hline & Bridge-Roadway-Width & $\mathrm{CDW}$ \\
\hline & Structure-Length & $\mathrm{BL}$ \\
\hline & Min-Vert-Clr-Over & VCO \\
\hline & Min-Vert-Clr-Under & $\mathrm{VCu}$ \\
\hline & Tot-Hz-Clr-Ov-En & $\mathrm{HCo}$ \\
\hline & Tot-Hz-Clr-Un-En & $\mathrm{HCu}$ \\
\hline & Skew & SKW \\
\hline & Substructure Height & SUBH (See VCu) \\
\hline & Number-Main-Spans & NU SPNS \\
\hline & Length-Max-Span & LGTH MAX SPN \\
\hline \multirow{5}{*}{$\begin{array}{l}\text { Bridge Type and } \\
\text { Construction }\end{array}$} & Main-Structure-Type & M STRTP \\
\hline & Struct-Matl & STR MTL \\
\hline & Struct-Const & STR CNST \\
\hline & Deck-Str-Type & DECK TP \\
\hline & Type-Wear-Surface & WS TP \\
\hline
\end{tabular}


Table G.2 IBMS Code Numbers for Bridge Improvements

\begin{tabular}{|c|c|}
\hline $\begin{array}{c}\text { ACTION } \\
\text { CODE }\end{array}$ & DECISION ACTION \\
\hline 0 & Do Nothing \\
\hline 1 & Deck Rehabilitation \\
\hline 2 & Deck Rehabilitation and Bridge Widening \\
\hline 3 & Deck Replacement \\
\hline 4 & Deck Replacement and Bridge Widening \\
\hline 5 & Deck and Superstructure Rehabilitation \\
\hline 6 & Deck and Superstructure Rehabilitation and Bridge Widening \\
\hline 7 & Deck Replacement and Superstructure Rehabilitation \\
\hline 8 & Deck Replacement, Superstructure Rehab and Bridge Widening \\
\hline 9 & Superstructure Rehabilitation \\
\hline 10 & Replace Superstructure \\
\hline 11 & Replace Superstructure and Bridge Widening \\
\hline 12 & Strengthen Superstructure \\
\hline 13 & Strengthen Superstructure and Bridge Widening \\
\hline 14 & Bridge Replacement \\
\hline 15 & Bridge Replacement and Bridge Widening \\
\hline 16 & Substructure Rehabilitation \\
\hline 17 & Raise Bridge/Lower Pavement (R/L) \\
\hline 18 & Substructure Rehabilitation with Full Depth Patching \\
\hline 19 & Culvert Replacement \\
\hline 20 & For later use \\
\hline 21 & Deck \& Sub Rehab $(1+16)^{1}$ \\
\hline 22 & Deck Replacement \& Sub Rehab $(3+16)$ \\
\hline 23 & Widen \& Replace Deck \& Sub Rehab (4+16) \\
\hline 24 & Deck \& Super \& Sub Rehab (5+16) \\
\hline
\end{tabular}

1. $(1+16)$ represents the combination of action 1 and action 16 .

Super: Superstructure

Sub: Substructure

Rehab: Rehabilitation

R/L Raise Bridge/ Lower Pavement

Source: Rodriguez 2004 ; Gion et al. 1993. 
Table G.2 IBMS Code Numbers for Bridge Improvements (Continued) ACTION

CODE

\section{DECISION ACTION}

\begin{tabular}{|c|c|}
\hline 25 & Deck Replacement \& Super \& Sub Rehab $(7+16)$ \\
\hline 26 & Widen \& Replace Deck \& Super \& Sub Rehab (8+16) \\
\hline 27 & Super Replace and Sub Rehab (9+16) \\
\hline 28 & Widen Deck \& Super Replace \& Sub Rehab $(11+16)$ \\
\hline 29 & Strengthen Super \& Sub Rehab $(12+16)$ \\
\hline 30 & Widen Deck \& Strengthen Super \& Rehab $(13+16)$ \\
\hline 31 & Deck Rehab \& Raise/Lower $(1+17)$ \\
\hline 32 & Deck Replace \& Raise/Lower $(3+17)$ \\
\hline 33 & Widen \& Replace Deck \& Raise/Lower (4+17) \\
\hline 34 & Deck \&Super Rehab \& Raise /Lower (5+17) \\
\hline 35 & Deck Replace \& Super Rehab \& Raise/Lower $(7+17)$ \\
\hline 36 & Widen \& Replace Deck \& Super Rehab \& R/L (8+17) \\
\hline 37 & Super Replacement \& Raise/ Lower $(10+17)$ \\
\hline 38 & Widen Deck \& Super Replacement \& R/L (11+17) \\
\hline 39 & Strengthen Super \& Raise/Lower $(12+17)$ \\
\hline 40 & Widen Deck \& Strengthen Super \& R/L (13+17) \\
\hline 41 & Deck \& Sub Rehab \& Raise/Lower (21+17) \\
\hline 42 & Deck Replacement \& Sub Rehab \& R/L (23 +17) \\
\hline 43 & Widen \& Replace Deck \& Sub Rehab \& R/L (23+17) \\
\hline 44 & Deck \& Super \& Sub Rehab \& R/L (24+17) \\
\hline 45 & Deck Replace \& Super \& Sub Rehab \& R/L (25+17) \\
\hline 46 & Widen \& Replace Deck \& Sup \& Sub Rehab \& R/L (26+17) \\
\hline 47 & Super Replacement \& Sub Rehab \& R/L (27+17) \\
\hline 48 & Widen Deck \& Super Replace \& Sub Rehab \& R/L (28+17) \\
\hline 49 & Strengthen Super \& Sub Rehab \& R/L (29+17) \\
\hline 50 & Widen Deck \& Strengthen Super \& Sub Rehab \& R/L (30+17) \\
\hline
\end{tabular}

Source: Rodriguez 2004 ; Gion et al. 1993. 
Table G.3 Bridge Preservation Cost Models

\begin{tabular}{|c|c|c|}
\hline No. & Activity & Cost prediction model \\
\hline 1 & Deck Rehabilitation (DH) & $\mathrm{DH}=\left(\text { Unit } \text { Cost }^{1}\right)^{*} \mathrm{DA}$ \\
\hline 2 & $\begin{array}{c}\text { Deck Rehabilitation \& Bridge } \\
\text { Widening (DH \&W) }\end{array}$ & $\mathrm{DH} \& \mathrm{~W}=\mathrm{DH}+1.2 *(\mathrm{SUPC}+\mathrm{SUBC})+0.6^{*} \mathrm{APC}+0.4 * \mathrm{OTHC}$ \\
\hline 3 & Deck Replacement (DR) & $\mathrm{DR}=35^{*} \mathrm{DA}$ \\
\hline 4 & $\begin{array}{l}\text { Deck Replacement \& bridge } \\
\text { Widening (DR \& W) }\end{array}$ & $\begin{array}{c}\mathrm{DR} \& \mathrm{~W}=1.0^{*} \mathrm{DR}+1.2^{*}\left(\mathrm{SUPC}_{\mathrm{w}}+\mathrm{SUBC}_{\mathrm{w}}\right)+0.6^{*} \mathrm{APC}+ \\
0.4 * \mathrm{OTHC}_{\mathrm{w}}\end{array}$ \\
\hline 5 & $\begin{array}{l}\text { Deck \& Superstructure } \\
\text { Rehabilitation }(\mathrm{D} \& \mathrm{SH})\end{array}$ & $\mathrm{D} \& \mathrm{SH}=1.1 * \mathrm{DH}$ \\
\hline 6 & $\begin{array}{c}\text { Deck \& Superstructure } \\
\text { Rehabilitation \& Bridge Widening } \\
\text { (D\&SH\&W) }\end{array}$ & $\begin{aligned} \mathrm{D} \& \mathrm{SH} \& \mathrm{~W}=1.1 * \mathrm{DH}+ & 1.2 *\left(\mathrm{SUPC}_{\mathrm{w}}+\mathrm{SUBC}_{\mathrm{w}}\right)+0.6^{*} \mathrm{APC}+ \\
& 0.4 * \mathrm{OTHC}^{\mathrm{O} T H}\end{aligned}$ \\
\hline 7 & $\begin{array}{c}\text { Deck Replacement \& } \\
\text { Superstructure Rehabilitation } \\
\text { (DR\&SH) }\end{array}$ & $\mathrm{DR} \& \mathrm{SH}=1.1 * \mathrm{DR}$ \\
\hline 8 & $\begin{array}{l}\text { Deck Replacement \& } \\
\text { Superstructure Rehabilitation \& } \\
\text { Bridge Widening (DR\&SH\&W) }\end{array}$ & $\mathrm{DR} \& \mathrm{SH} \& \mathrm{~W}=0.85^{*} \mathrm{BR}$ \\
\hline 9 & Superstructure Rehabilitation & $\mathrm{SH}=0.2 \mathrm{DH}$ \\
\hline 10 & Replace Superstructure & $\mathrm{SR}=\mathrm{SUPC}+0.4^{*} \mathrm{APC}+\mathrm{OTHC}$ \\
\hline 11 & $\begin{array}{c}\text { Superstructure Replacement \& } \\
\text { Bridge Widening (SR\&W) }\end{array}$ & $\mathrm{SR} \& \mathrm{~W}=\mathrm{SUPC}+0.5 * \mathrm{SUBC}+0.7 * \mathrm{APC}+\mathrm{OTHC}$ \\
\hline 12 & Strengthen Superstructure (ST) & $\mathrm{ST}=\mathrm{BL} * \mathrm{DW} *(30-0.0001 * \mathrm{BL} * \mathrm{WL})$ \\
\hline 13 & $\begin{array}{l}\text { Strengthen Superstructure \& } \\
\text { Bridge Widening (ST\&W) }\end{array}$ & $\mathrm{ST} \& \mathrm{~W}=\mathrm{ST}+1.2^{*}\left(\mathrm{SUPC}_{\mathrm{w}}+\mathrm{SUBC}_{\mathrm{w}}\right)+0.6 * \mathrm{APC}+0.4 * \mathrm{OTHC}$ \\
\hline 14 & Bridge Replacement & $\mathrm{BR}=\mathrm{SUPC}+\mathrm{SUBC}+\mathrm{APC}+\mathrm{OTHC}$ \\
\hline 15 & $\begin{array}{l}\text { Bridge Replacement \&Bridge } \\
\text { Widening }\end{array}$ & $\mathrm{BR}=\mathrm{SUPC}_{\mathrm{w}}+\mathrm{SUBC}_{\mathrm{w}}+\mathrm{APC}+\mathrm{OTHC}$ \\
\hline 16 & Substructure Rehabilitation $(\mathrm{BH})$ & $\mathrm{BH}=10 * \mathrm{BL} * \mathrm{DW}$ \\
\hline 17 & $\begin{array}{c}\text { Raise Bridge/Lower Pavement } \\
(\mathrm{R} / \mathrm{L}) \\
\end{array}$ & $\mathrm{R} / \mathrm{L}=0.803\left[12 *\left(\mathrm{DVC}_{\mathrm{u}}-\mathrm{VC}_{\mathrm{U}}\right)\right]^{1.3 * \mathrm{BL} * \mathrm{DW}}$ \\
\hline 18 & $\begin{array}{l}\text { Deck Rehabilitation with Full } \\
\text { Depth Patching (DH\&P) }\end{array}$ & $\mathrm{DH} \& \mathrm{P}=1.1 * \mathrm{DH}$ \\
\hline 19 & Culvert Replacement (CR) & CR $=$ Culvert Cost $^{2}$ \\
\hline \multicolumn{3}{|c|}{ Source: Rodriguez 2004; Vitale 1997} \\
\hline \multicolumn{3}{|c|}{ APC: $\quad$ Approach Cost } \\
\hline \multicolumn{3}{|c|}{ OTHC: $\quad$ Other Cost } \\
\hline \multicolumn{2}{|r|}{ Deck Area } & \\
\hline \multicolumn{2}{|c|}{ DVCu: $\quad$ Desirable vertical clea } & e in feet (from clear subroutine) \\
\hline \multicolumn{3}{|c|}{ VCu: $\quad$ Vertical clearance under the bridge } \\
\hline SUPCw & /SUBCw: Superstructure/ subst & ure replacement cost using widened portion of width \\
\hline
\end{tabular}




\section{Appendix H. Highway Disbursements Analysis for Indiana Counties}

This appendix presents the procedure used to estimate the disbursement for Indiana counties on the basis of past trends. The following statistical relations were used:

$$
V M T=\mathrm{f}(\mathrm{Year})
$$

Disbursements $=\mathrm{f}(V M T)$

\section{H.1 VMT as a Function of Time}

First, the VMT for local roads was plotted against the years, using data from Highway Statistics (BTS 1991-2002). A statistical relation was estimated that enables the determination of VMT for any given year in future, as follows:

$$
V M T=269.34(Y E A R)-529,460
$$

Where VMT is vehicle miles of travel in millions

The observed data and the plot are shown in Figure H-1. Using the developed equation, the VMT's for each future year was determined through extrapolation, until the year 2019. It can be seen that the total VMT on Indiana's local roads increases by 269 million vehicle-miles per year. The annual rate of growth can be computed using the formula:

$\mathrm{r}=(\log (\mathrm{F})-\log (\mathrm{P})) /$ period of interest

where F represents the value of the VMT at the end of the period and P, the value of the VMT at the beginning of the period.

An annual rate of growth of $3 \%$ can be found. 


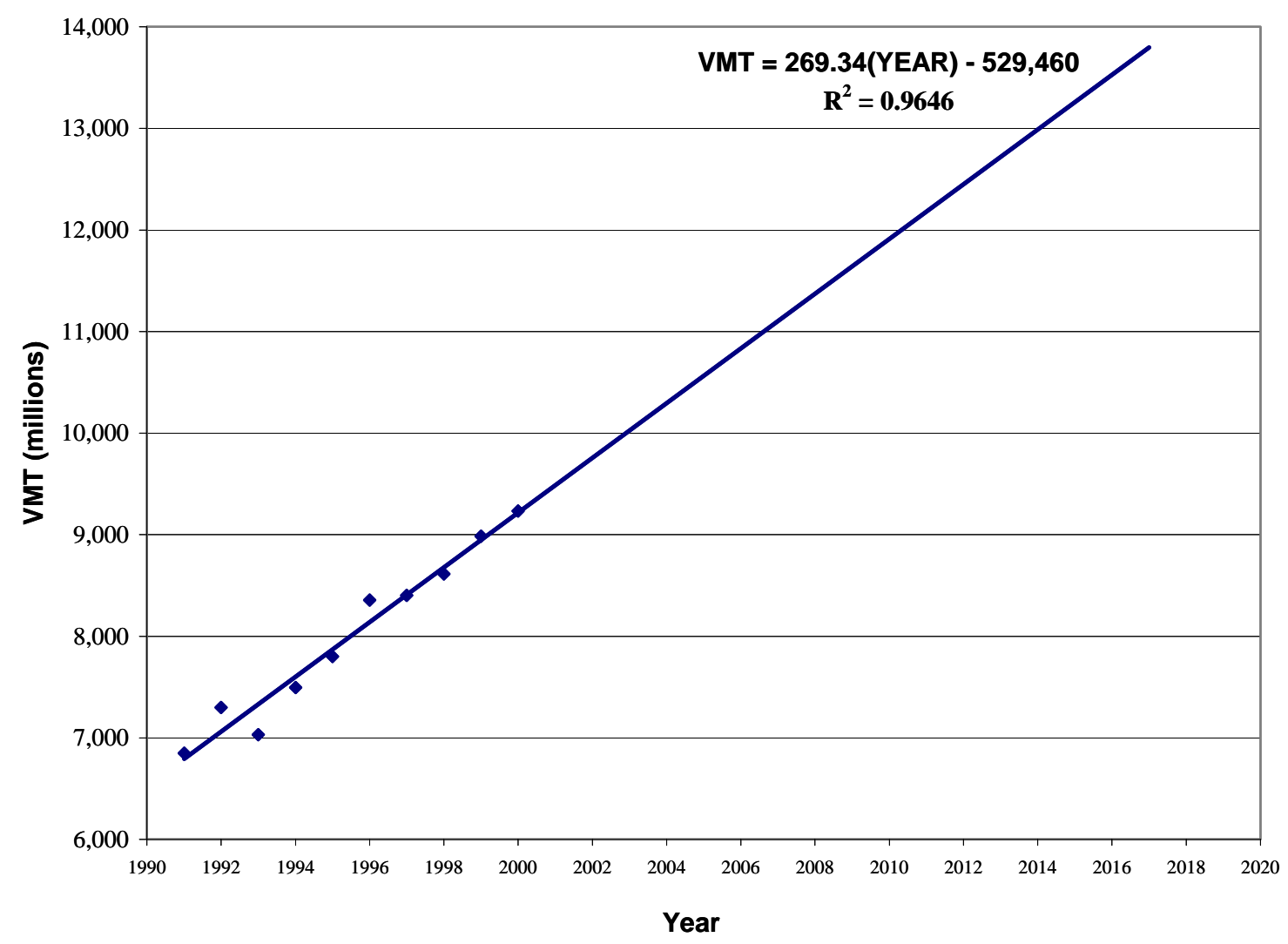

Figure H- 1: VMT versus Year

\section{H.2 Disbursement as a Function of Time}

Capital outlay and maintenance disbursements were also plotted against time, using data from Highway Statistics (BTS 1991-2001). The observed data is shown in Figure H-2. 


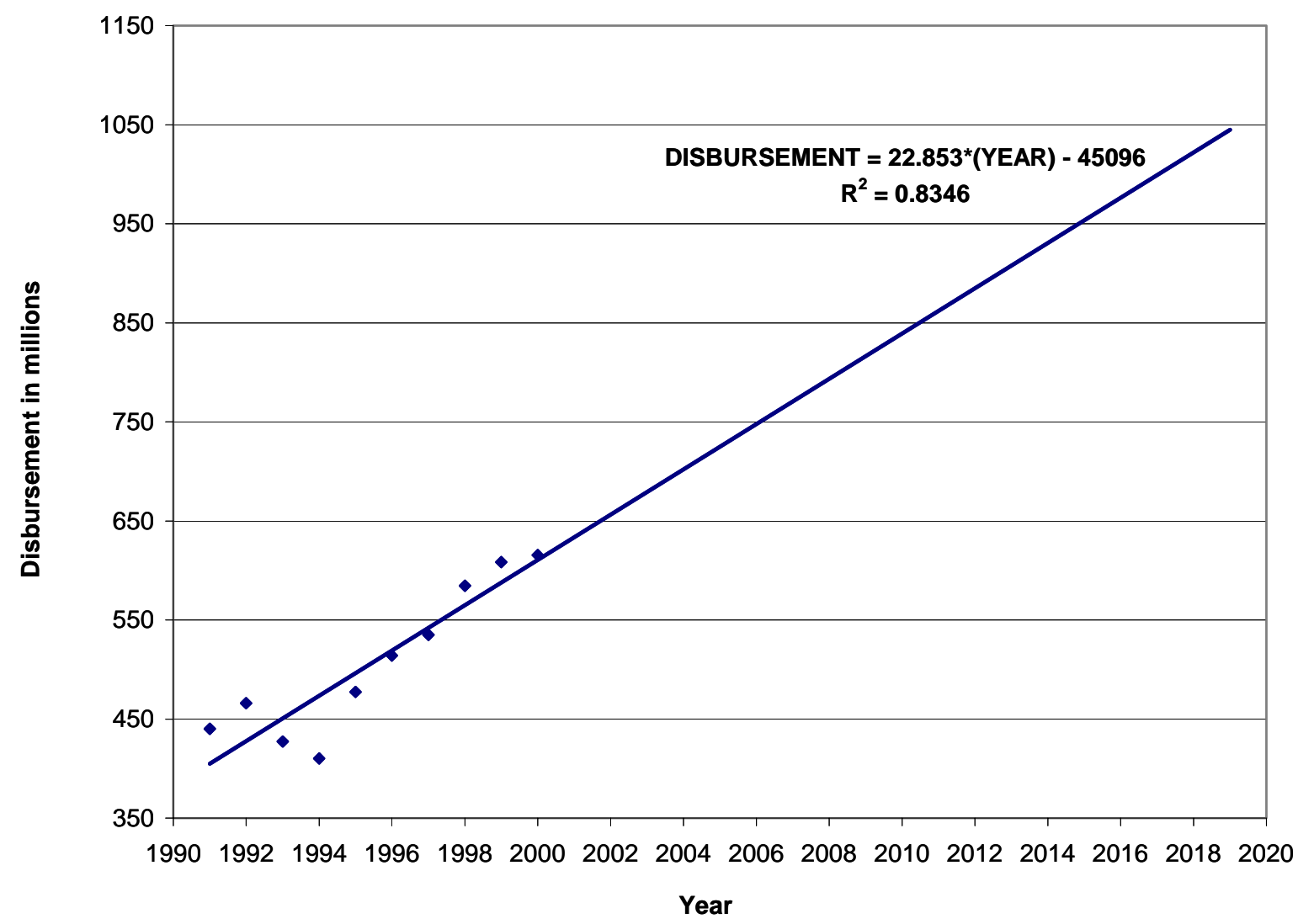

Figure H-2: Capital Outlay and Maintenance Disbursement versus Year (constant 2002 dollars)

Except for some fluctuations from 1991 to 1994, the level of disbursement on capital outlay and maintenance was stable.

\section{H.3 Disbursements as a Function of VMT}

The total disbursement was then determined as a function of vehicle-miles traveled, using data from Highway Statistics (BTS 1995-2001). The observed values and plot are shown in Figure H-3 in constant 2002 dollars, and it can be seen that the capital outlay and maintenance disbursement for Indiana counties is 0.119 dollars for each vehicle mile traveled. 


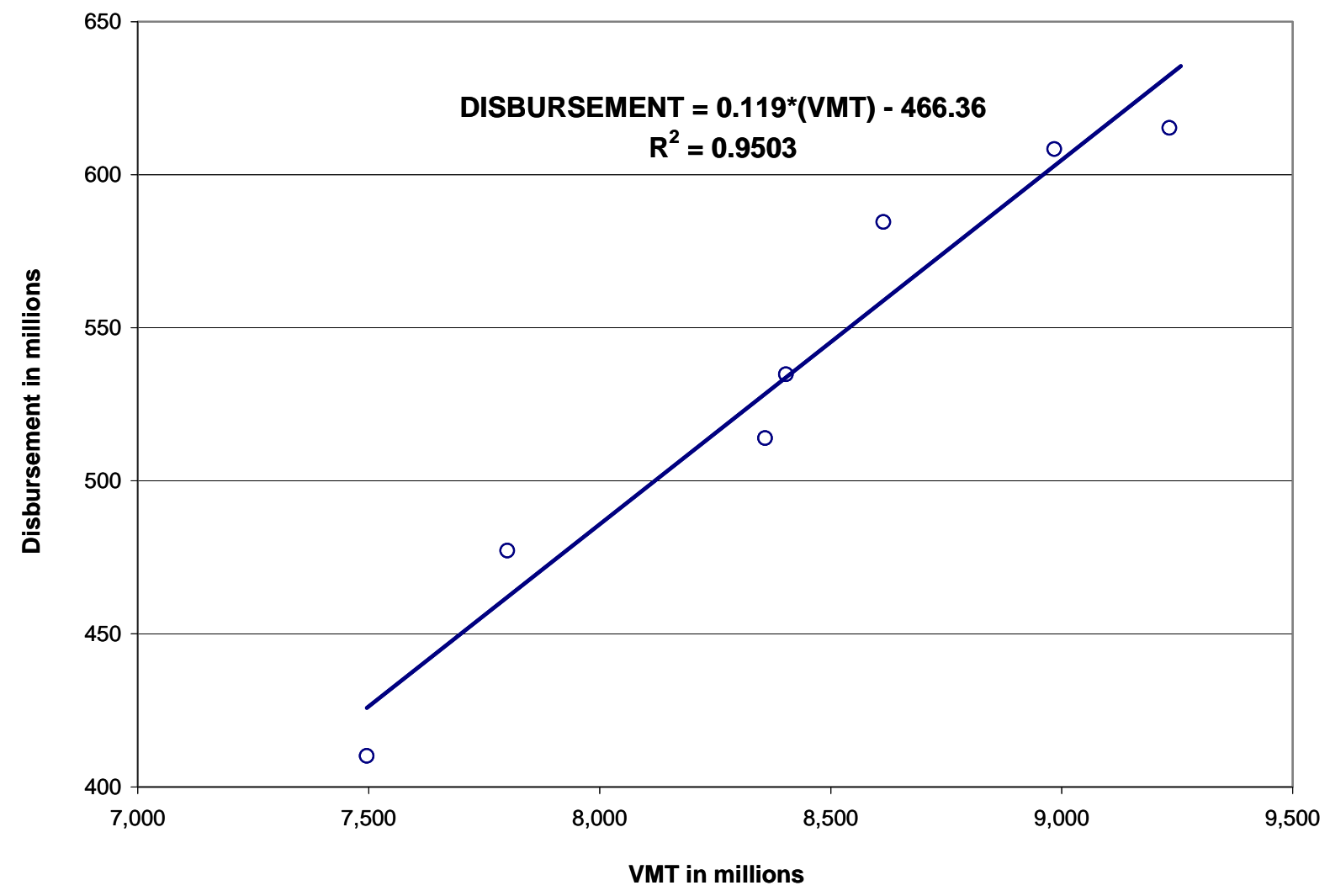

Figure H-3 : Disbursements versus VMT (constant2002 dollars)

A statistical relation was estimated that enables the determination of DISBURSEMENT for any given year, as follows:

DISBURSEMENT $=0.119($ VMT $)-466.36$

Where DISBURSEMENT is in millions of dollars,

$V M T$ is vehicle miles of travel on local roads in millions.

Using Figure H-3 or Equation (2), it is possible to find the expected level of future disbursement corresponding to any expected level of VMT for the forecasted years. 


\section{H.4 Disbursement per VMT as a Function of Time}

The disbursement per VMT was determined using data from Highway Statistics publications (BTS 1995-2001) for the capital outlay and maintenance disbursements and the VMT data from INDOT. The observed values of expenditure per VMT are shown on Figure H-4 in constant 2002 dollars. It can be seen that the overall level of disbursement has been increasing despite a slight decrease for the years 1999 and 2000.

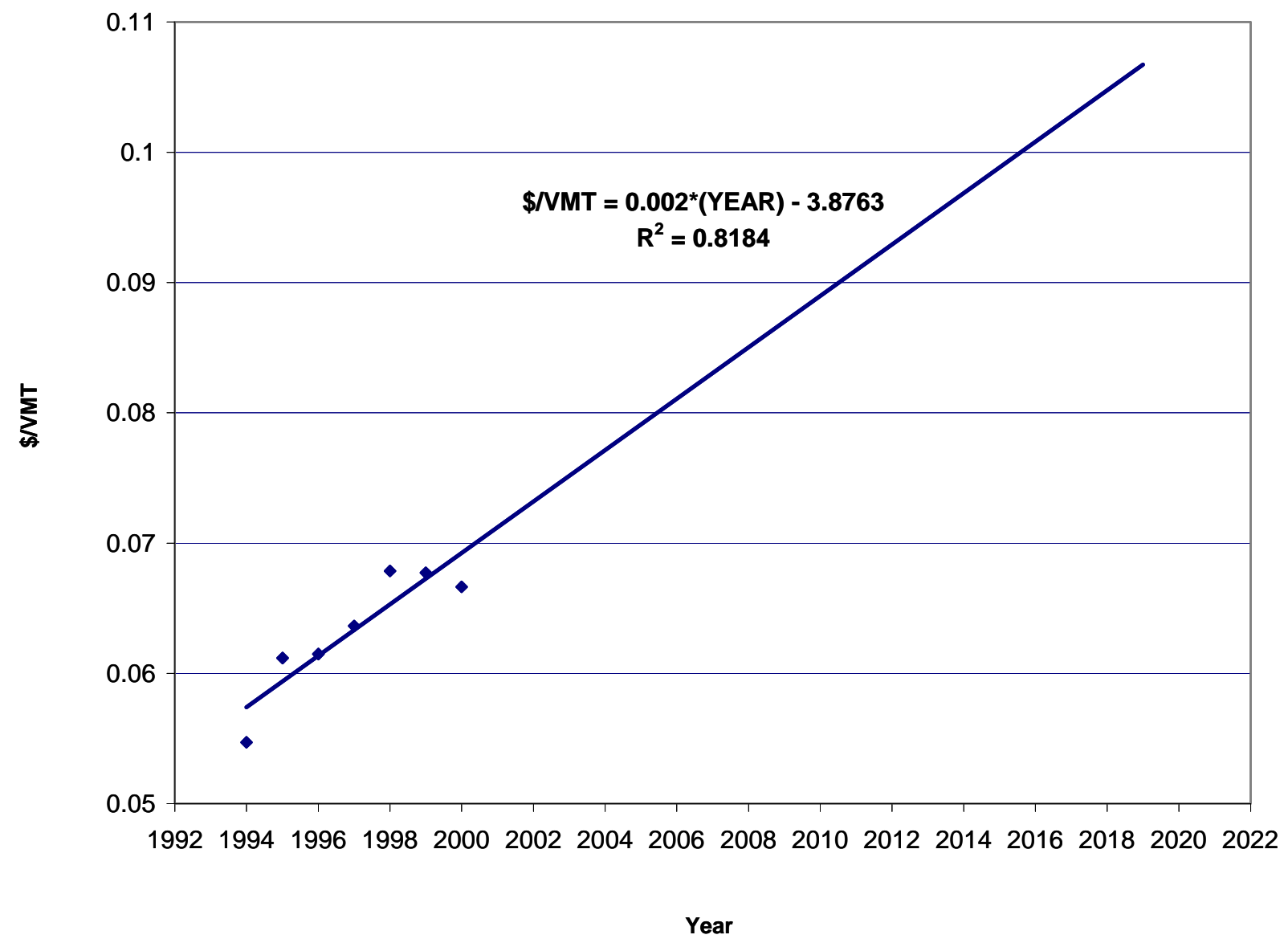

Figure H- 4: Capital Outlay and Maintenance Disbursements per VMT versus Year (2002 \$)

However, while the level of disbursement per VMT on capital outlay and maintenance has been increasing, the total disbursement per VMT has been decreasing in general. The observed data is plotted in Figure H-5. Because of high variability of the data, no statistically significant equation could be developed. 


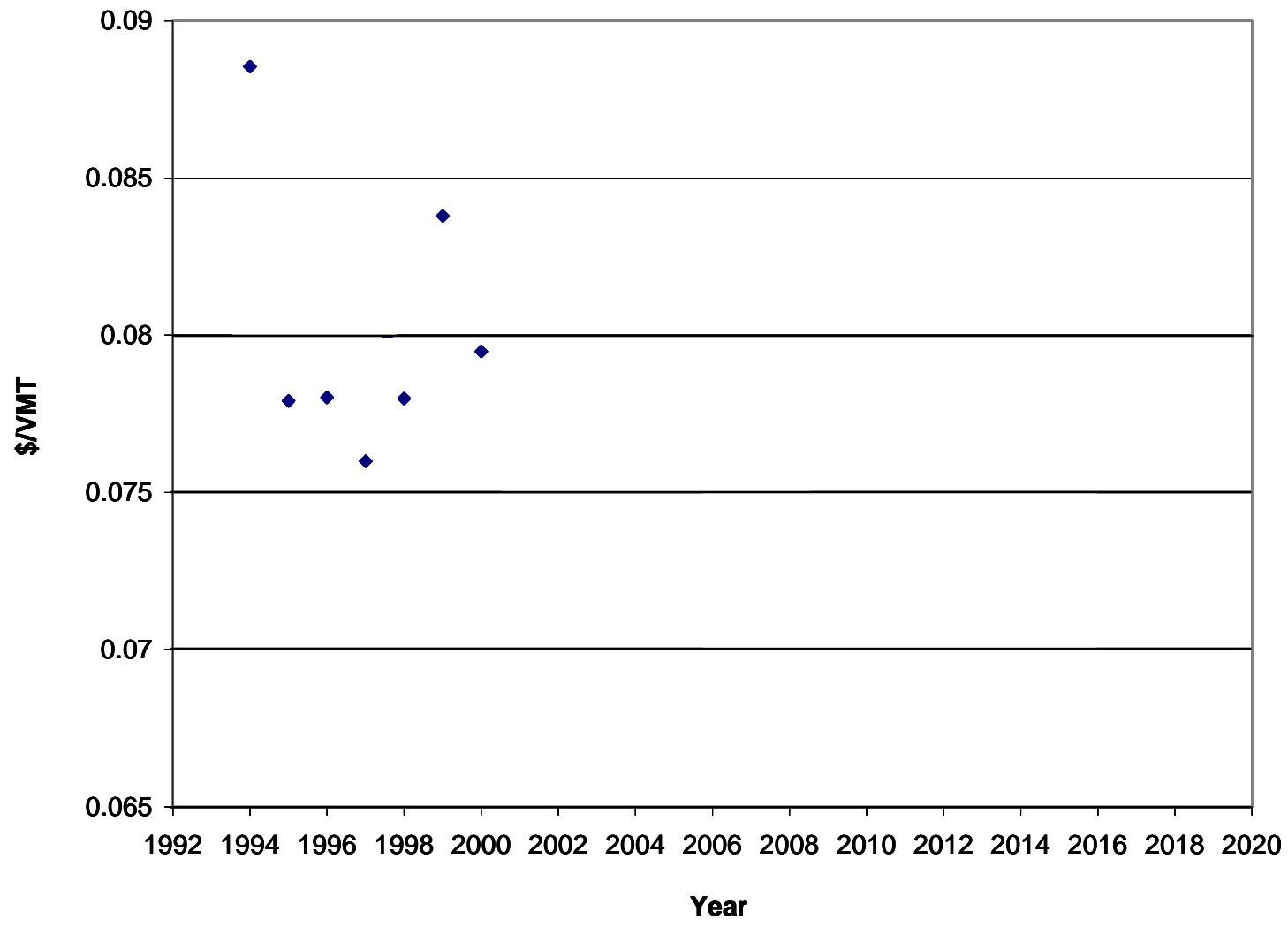

Figure H-5: Total Disbursements per VMT versus Year (constant 2002 dollars)

From these results we can conclude that, even though the counties have been trying to keep up with the level of basic maintenance for roads and streets, they are experiencing a shortfall in their overall level of funding. This implies that there is a severe shortfall when dealing with the money spent on administrative tasks, highway law enforcement and safety, bond retirement and transfer to sate governments. Table H-1 shows the capital outlay and maintenance disbursements projection from 2006 to 2020 in million dollars. 
Table H-1: Projected Disbursements (Capital Outlay and Maintenance) (2002 \$)

\begin{tabular}{|c|c|c|c|c|c|}
\hline Year & VMT & $\begin{array}{c}\text { Disbursement } \\
\text { in current } \\
\text { dollars (Million } \\
\$ \text { \$) }\end{array}$ & CPI & $\begin{array}{c}\text { Disbursement } \\
\text { in constant } \\
2002 \text { dollars } \\
\text { (Million \$) } \\
\end{array}$ & \$/VMT \\
\hline 1994 & 7,496 & 319 & 115.1 & 410 & 0.0547 \\
\hline 1995 & 7,800 & 393 & 121.9 & 477 & 0.0611 \\
\hline 1996 & 8,358 & 417 & 120.2 & 514 & 0.0614 \\
\hline 1997 & 8,403 & 472 & 130.6 & 534 & 0.0636 \\
\hline 1998 & 8,614 & 501 & 126.9 & 558 & 0.0678 \\
\hline 1999 & 8,984 & 561 & 136.5 & 608 & 0.0677 \\
\hline 2000 & 9,223 & 605 & 145.6 & 615 & 0.0666 \\
\hline 2001 & 9,489 & 649 & 144.8 & 663 & 0.0698 \\
\hline 2002 & 9,758 & 695 & 147.9 & 695 & 0.0712 \\
\hline 2003 & 10,028 & 752 & 153.0 & 726 & 0.0724 \\
\hline 2004 & 10,297 & 807 & 157.2 & 759 & 0.0737 \\
\hline 2005 & 10,566 & 863 & 161.3 & 791 & 0.0748 \\
\hline 2006 & 10,836 & 921 & 165.5 & 823 & 0.0759 \\
\hline 2007 & 11,105 & 981 & 169.7 & 855 & 0.0770 \\
\hline 2008 & 11,374 & 1,043 & 173.9 & 887 & 0.0780 \\
\hline 2009 & 11,644 & 1,107 & 178.1 & 919 & 0.0789 \\
\hline 2010 & 11,913 & 1,172 & 182.2 & 951 & 0.0798 \\
\hline 2011 & 12,182 & 1,239 & 186.4 & 983 & 0.0807 \\
\hline 2012 & 12,452 & 1,309 & 190.6 & 1,015 & 0.0815 \\
\hline 2013 & 12,721 & 1,380 & 194.8 & 1,047 & 0.0823 \\
\hline 2014 & 12,990 & 1,452 & 199.0 & 1,079 & 0.0831 \\
\hline 2015 & 13,260 & 1,527 & 203.1 & 1,111 & 0.0838 \\
\hline 2016 & 13,529 & 1,063 & 207.3 & 1,143 & 0.0845 \\
\hline 2017 & 13,798 & 1,681 & 211.5 & 1,175 & 0.0852 \\
\hline 2018 & 14,068 & 1,761 & 215.7 & 1,207 & 0.0858 \\
\hline 2019 & 14,337 & 1,843 & 219.9 & 1,239 & 0.0864 \\
\hline 2020 & 14,607 & 1,927 & 224.1 & 1,272 & 0.0870 \\
\hline
\end{tabular}


Appendix I. Local Bridge Sufficiency Ratings by County

Area All Percent Percent Percent $\begin{array}{ccccc}\text { Number of } & \text { Bridges } & \text { with S.R. } & \text { with S R. } & \text { with S.R } \\ \text { B ridges } & \text { (sq. } \pi \text { ) } & 0-43.9 & 50-19.9 & 80-100\end{array}$

Code County

\begin{tabular}{|c|c|c|c|}
\hline 240,409 & $6.7 \%$ & $14.1 \%$ & $79.2 \%$ \\
\hline $1,022,497$ & $6.6 \%$ & $27.7 \%$ & B.5.7\% \\
\hline 564,400 & $19.3 \%$ & $29.8 \%$ & $56.9 \%$ \\
\hline 162,778 & 17.086 & $0.9 \%$ & $74.19 \mathrm{t}$ \\
\hline 78,027 & $1.8 \%$ & $7.1 \%$ & $01.1 \%$ \\
\hline 295,866 & $18.8 \%$ & $17.1 \%$ & B4.1\% \\
\hline 85,100 & $30.7 \%$ & $37.5 \%$ & $31.8 \%$ \\
\hline 256,368 & $17.5 \%$ & $19.3 \%$ & $63.2 \%$ \\
\hline 287,668 & $10.2 \%$ & $6.8 \%$ & B3.1\% \\
\hline 194,411 & $1.7 \%$ & $21.7 \%$ & $76.7 \%$ \\
\hline 179,142 & $30.1 \%$ & $29.2 \%$ & $96.7 \%$ \\
\hline 252,339 & $16.1 \%$ & $29.9 \%$ & $60.0 \% t$ \\
\hline 101,640 & $63.0 \%$ & $26.0 \%$ & $11.0 \%$ \\
\hline 177,489 & $13.7 \%$ & $12.1 \%$ & $74.2 \%$ \\
\hline 195,287 & $27.8 \%$ & $27.8 \%$ & $44.4 \%$ \\
\hline 256,162 & $14.4 \%$ & $29.3 \%$ & $56.3 \%$ \\
\hline 196,927 & $3.1 \%$ & $7.1 \%$ & $89.8 \%$ \\
\hline 56,769 & $18.9 \%$ & $18.9 \%$ & B2.1\% \\
\hline 193,955 & $10 . \%$ & $22.1 \%$ & $67.6 \%$ \\
\hline 403,169 & $9.1 \%$ & $23.1 \%$ & $79.0 \%$ \\
\hline 194,154 & $11.8 \%$ & $16.5 \%$ & $71.89 t$ \\
\hline 139,820 & $28.4 \%$ & $19.8 \%$ & $51.9 \%$ \\
\hline 232,179 & $21.8 \%$ & $20.4 \%$ & $57.7 \%$ \\
\hline 231,077 & $32.0 \%$ & $35.0 \%$ & $33.0 \%$ \\
\hline 110,09 & $6.8 \%$ & $13.6 \%$ & $79.7 \%$ \\
\hline 287,062 & $25.2 \%$ & $36.8 \%$ & $38.0 \%$ \\
\hline 340,024 & $6.4 \%$ & $27.1 \%$ & $66.5 \%$ \\
\hline 210,462 & $29.7 \%$ & $25.3 \%$ & $44.9 \%$ \\
\hline 604,922 & $11.4 \%$ & $29.3 \%$ & $65.39 \mathrm{t}$ \\
\hline 279,701 & $2.1 \%$ & $16.9 \%$ & $81.0 \% t$ \\
\hline $121,7 \pi$ & $18.8 \%$ & $10.1 \%$ & $71.0 \%$ \\
\hline 426,913 & $5.3 \%$ & $24.9 \%$ & $69.8 \%$ \\
\hline 217,886 & $13.1 \%$ & $16.9 \%$ & $70.0 \%$ \\
\hline 316,155 & $3.8 \%$ & $43.2 \%$ & $53.0 \%$ \\
\hline 259.600 & $6.2 \%$ & $11.5 \%$ & $62.3 \%$ \\
\hline 305,737 & $16.9 \%$ & $12.4 \%$ & $70.8 \%$ \\
\hline 230,659 & $10.2 \%$ & $7.8 \%$ & B2.0\% \\
\hline $240,4 \pi 0$ & $0.0 \%$ & $22.6 \%$ & $60.6 \%$ \\
\hline 164,221 & $19.0 \%$ & $26.0 \%$ & $55.0 \% \mathrm{t}$ \\
\hline 222,990 & 33.986 & $24.4 \%$ & $41.7 \%$ \\
\hline 247,181 & $5.1 \%$ & $19.6 \%$ & $75.4 \%$ \\
\hline 348,052 & $22.1 \%$ & $34.3 \%$ & $43.7 \%$ \\
\hline 144,326 & $1.9 \%$ & $12.6 \%$ & B5.4\% \\
\hline 92,127 & $1.9 \%$ & $13.5 \%$ & $64.6 \%$ \\
\hline 642,945 & $20.1 \%$ & $27.7 \%$ & $52.2 \%$ \\
\hline 164,753 & $3.5 \%$ & $14.9 \%$ & $61.6 \%$ \\
\hline
\end{tabular}

\begin{tabular}{|c|c|c|}
\hline Code & County & $\begin{array}{c}\text { Number a } \\
\text { Bridges }\end{array}$ \\
\hline 47 & Lamxence & $1 \$ 1$ \\
\hline 40 & Madison & 195 \\
\hline 49 & Marian & 512 \\
\hline 50 & Marshal & 110 \\
\hline 51 & Martin & 46 \\
\hline 52 & Mismi & 130 \\
\hline 53 & Monroe & 130 \\
\hline 54 & Montgomery & 172 \\
\hline 55 & M organ & 141 \\
\hline 56 & Newton & 121 \\
\hline 57 & Noble & 60 \\
\hline 58 & Ohio & 21 \\
\hline 59 & Orange & 104 \\
\hline 60 & Owen & 115 \\
\hline 61 & $\mathrm{P} s \boldsymbol{x} \mathrm{k} \theta$ & 180 \\
\hline 62 & Perry & 100 \\
\hline 63 & Pike & 112 \\
\hline 64 & Porter & 124 \\
\hline 65 & $P$ cosey & 157 \\
\hline 66 & $P$ una & 79 \\
\hline 67 & Putnam & 219 \\
\hline 68 & Rendolph & 220 \\
\hline 69 & Ripley & 131 \\
\hline 70 & Rush & 192 \\
\hline 71 & St. Joseph & 8 \\
\hline 72 & Soott & 70 \\
\hline 73 & Shelby & 183 \\
\hline 74 & Sperer & 170 \\
\hline 75 & Starke & 69 \\
\hline 76 & Steuken & 48 \\
\hline 77 & Sullivan & 179 \\
\hline 78 & Switzerland & 40 \\
\hline 79 & Tippecanoe & 169 \\
\hline 80 & Tipton & $\infty$ \\
\hline 81 & Union & 43 \\
\hline 82 & Vanderburth & 147 \\
\hline 83 & vermillion & 75 \\
\hline 64 & Vigo & 190 \\
\hline 85 & Wabash & 152 \\
\hline$\theta 6$ & Warren & 97 \\
\hline $8 T$ & Werrick & 111 \\
\hline 88 & Westington & 133 \\
\hline 89 & Wayne & 229 \\
\hline 90 & Wells & 134 \\
\hline 91 & White & 156 \\
\hline 92 & Whitley & $\sigma 2$ \\
\hline
\end{tabular}

Area Al Percent Percent Percent Bridges with SR. with S.R. with S.R. (80 II) 0-49.9 50-79.9 80-100

\begin{tabular}{|c|c|c|c|}
\hline 296,706 & $51,3 \%$ & $16.8 \%$ & $51.9 \%$ \\
\hline 616,607 & $02 \%$ & $12.6 \%$ & $79.1 \%$ \\
\hline $3,555,094$ & $90 \%$ & 42.246 & $48.8 \%$ \\
\hline 214,569 & $0.9 \%$ & 4.546 & $94.6 \%$ \\
\hline $5 B, 712$ & $319 \%$ & $36.2 \%$ & $31.9 \%$ \\
\hline 257,745 & $143 \%$ & $23.8 \%$ & $61.9 \%$ \\
\hline 220,529 & $115 \%$ & $25.4 \%$ & $63.1 \%$ \\
\hline 390,052 & $15.1 \%$ & $8.1 \%$ & $76.7 \%$ \\
\hline 247,393 & $270 \%$ & $20.6 \%$ & $52.5 \%$ \\
\hline 169,696 & $63 \%$ & $19.0 \%$ & $72.7 \%$ \\
\hline 69,002 & $0.3 \%$ & $20.0 \%$ & $71.7 \%$ \\
\hline 26,139 & $22.7 \%$ & 18.246 & $59.1 \%$ \\
\hline 137,284 & $38.7 \%$ & $19.8 \%$ & $41.5 \%$ \\
\hline 182,480 & $429 \%$ & $17.0 \%$ & $40.2 \%$ \\
\hline 284,444 & $22.9 \%$ & $34.1 \%$ & $43.0 \%$ \\
\hline 115,539 & $186 \%$ & $35.3 \%$ & $46.1 \%$ \\
\hline $12 B, 451$ & $170 \%$ & $22.3 \%$ & $60.7 \%$ \\
\hline 234,963 & $49 \%$ & $36.9 \%$ & $58.2 \%$ \\
\hline 200,900 & $25.3 \%$ & $16.9 \%$ & $57.0 \%$ \\
\hline 179,776 & $6.6 \%$ & $5.3 \%$ & $60.2 \%$ \\
\hline 339,390 & $436 \%$ & 20.046 & $36.4 \%$ \\
\hline 375,418 & $150 \%$ & $16.4 \%$ & $68.6 \%$ \\
\hline 175,069 & $13.7 \%$ & $27.5 \%$ & $58.8 \%$ \\
\hline $26 \mathrm{~B}, 103$ & $93 \%$ & $21.2 \%$ & $69.4 \%$ \\
\hline 580,233 & $136 \%$ & $22.7 \%$ & $63.6 \%$ \\
\hline 64,076 & $113 \%$ & $25.4 \%$ & $63.4 \%$ \\
\hline 376,355 & $232 \%$ & $24.9 \%$ & $51.9 \%$ \\
\hline 162,644 & $112 \%$ & $27.2 \%$ & $61.5 \%$ \\
\hline$\theta 0,091$ & $39.7 \%$ & 39.346 & $27.0 \%$ \\
\hline 66,992 & $00 \%$ & 4.246 & $95.6 \%$ \\
\hline 228,407 & $220 \%$ & $33.9 \%$ & $44.1 \%$ \\
\hline 51,161 & $425 \%$ & $27.5 \%$ & $30.0 \%$ \\
\hline 477,288 & $4.7 \%$ & $16.5 \%$ & $78.8 \%$ \\
\hline 129,402 & $89 \%$ & $13.9 \%$ & $77.2 \%$ \\
\hline 94,796 & $2.3 \%$ & $11.6 \%$ & $86.0 \%$ \\
\hline 440,845 & $20 \%$ & $22.4 \%$ & $75.5 \%$ \\
\hline 161,712 & $120 \%$ & $16.0 \%$ & $72.0 \%$ \\
\hline 930,122 & $96 \%$ & $26.2 \%$ & $64.2 \%$ \\
\hline 322,790 & $3.3 \%$ & 13.846 & $82.9 \%$ \\
\hline $174,72 \theta$ & $26.8 \%$ & 16.546 & $56.7 \%$ \\
\hline 214,749 & $99 \%$ & $10.8 \%$ & $79.3 \%$ \\
\hline 211,528 & $46 \%$ & $21.4 \%$ & $74.0 \%$ \\
\hline 661,318 & $15.7 \%$ & $17.0 \%$ & $67.4 \%$ \\
\hline 213.518 & $3.1 \%$ & $19.5 \%$ & $77.3 \%$ \\
\hline 324,276 & $1.3 \%$ & $8.9 \%$ & $89.9 \%$ \\
\hline 120,097 & $16.7 \%$ & $40.5 \%$ & $42.9 \%$ \\
\hline
\end{tabular}


Appendix J. Cost Estimations for Local Bridge Replacement

\begin{tabular}{|c|c|c|c|c|c|c|}
\hline Code & County & $\begin{array}{r}\text { Area All } \\
\text { Bridges } \\
\text { (3q. ft) }\end{array}$ & $\begin{array}{r}\text { Percent } \\
\text { with S.R. } \\
\text { D-49.9 }\end{array}$ & $\begin{array}{r}\text { Area Bridges } \\
\text { with SR } \\
\text { D }-49.9\end{array}$ & $\begin{array}{r}\text { Unit cost } \\
\text { Bridge } \\
\text { Replacement }\end{array}$ & $\begin{array}{r}\text { Total cost } \\
\text { Bridge } \\
\text { Replacement }\end{array}$ \\
\hline & & & & (sq. $\mathrm{ft}$ & 6/sq. fi. & $2 \mathrm{DO} 2$ dolla re \\
\hline 1 & Adams & 229544 & $19 \%$ & 4311 & \$106 & $\$ 456948$ \\
\hline 2 & Allen & $10770 \mathrm{0B} 7$ & $50 \%$ & 54,223 & $\$ 106$ & 86,747627 \\
\hline 3 & Barihalomew & 574,700 & $6.3 \%$ & 36,379 & $\$ 106$ & B $3,866,140$ \\
\hline 4 & Benton & 162,412 & $12.4 \%$ & 20,197 & $\$ 106$ & $\$ 2,140917$ \\
\hline 5 & Blackford & 79662 & $32 \%$ & 2578 & $\$ 106$ & 3273,301 \\
\hline 6 & Boone & 298524 & $119 \%$ & 35,512 & $\$ 106$ & $5,7642 \mathrm{EO}$ \\
\hline 7 & Brown & 85,153 & $2 \mathrm{E} .4 \%$ & 22,462 & $\$ 106$ & 52,380978 \\
\hline 日 & Carmll & 2559553 & $106 \%$ & $2 \pi, 170$ & $\$ 105$ & $\sigma 2, \boldsymbol{⿴ 囗 十 力}, 055$ \\
\hline 9 & Cass & $2876 \mathrm{~B} 8$ & $73 \%$ & 21,139 & $\$ 106$ & $82,240,773$ \\
\hline 10 & Clark & 200518 & $1.7 \%$ & 3,332 & $\$ 106$ & $\$ \times 5,155$ \\
\hline 11 & Clay & 185913 & $242 \%$ & 45,053 & $\$ 106$ & $64,775,6 \square 4$ \\
\hline 12 & Clintan & 247231 & $\begin{array}{l}160 \% \\
160 \%\end{array}$ & 思, & $\$ 106$ & 54,7187 \\
\hline 13 & Crawtord & 101.434 & $35.4 \%$ & 35,925 & $\$ 106$ & $53,600,0 \mathrm{E} 6$ \\
\hline 14 & Dostiess & 171,747 & $142 \%$ & 24,441 & $\$ 106$ & $52,90,778$ \\
\hline 15 & Desibom & $1950 \mathrm{DE} 2$ & $159 \%$ & 31,060 & $\$ 106$ & $50,294,429$ \\
\hline 16 & Decetur & 255,773 & $112 \%$ & $\mathbb{7}, 5 \mathbb{7}$ & $\$ 106$ & 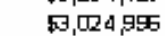 \\
\hline 17 & Dekalb & 197,732 & $30 \%$ & 6 D18 & $\$ 106$ & 9637878 \\
\hline 18 & Delamere & 575867 & $143 \%$ & $B \mathrm{~B}, 345$ & $\$ 106$ & 田, 72 日E \\
\hline 19 & Dubois & 191,729 & B.0\% & 11,520 & $\$ 106$ & $\mathbf{5 1 , 2 2 1 , 0 9 9}$ \\
\hline 20 & Elkhert & $497,47 \theta$ & $9.4 \%$ & 46,720 & $\$ 106$ & $54,952,399$ \\
\hline 21 & Fayetta & 193876 & $50 \%$ & 9726 & $\$ 106$ & $\$ 1,030971$ \\
\hline 27 & Floyd & 142092 & $20.7 \%$ & 20,446 & $\$ 10 E$ & $\mathrm{~B}, 121, \mathrm{~g} 9$ \\
\hline 20 & Fountain & 226078 & $20.5 \%$ & 45,451 & $\$ 106$ & Ed, $, 023,774$ \\
\hline 24 & Franklin & 232,039 & B. $4 \%$ & 19,551 & $\$ 106$ & $62,072,406$ \\
\hline 둗 & Fulton & 110597 & $\theta 0 \%$ & घ901 & $\$ 106$ & 59435 5교 \\
\hline$\overline{26}$ & Gibsan & 290671 & $20.1 \%$ & 58,484 & $\$ 106$ & $56,199,2 \mathrm{0}$ \\
\hline 27 & Grant & $33 \mathrm{\theta} 972$ & $9.4 \%$ & 31,999 & $\$ 106$ & 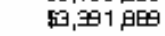 \\
\hline 28 & Graene & 207633 & $19.4 \%$ & 40,284 & $\$ 106$ & $64,270,0 \mathrm{EP}$ \\
\hline 20 & Hamitton & 632,155 & $42 \%$ & 26,767 & $\$ 106$ & $52,837,321$ \\
\hline 30 & Hancock & 279554 & $13 \%$ & $3 \mathrm{~B} 03$ & $\$ 106$ & $\$ 381,899$ \\
\hline 31 & Harrison & 129044 & $76 \%$ & $9 B 03$ & \$106 & \$1,039073 \\
\hline 크 & Hendricks & 426996 & $53 \%$ & $2,4 \exists 1$ & $\$ 105$ & $5, \exists 7,7 \square$ \\
\hline 39 & Henry & $21 \mathrm{~B}, 5 \mathrm{D} 9$ & $\mathrm{~B} 6 \%$ & 14,348 & $\$ 106$ & $\mathrm{~S} 1,52 \mathrm{Q}, 8 \mathrm{E} 2$ \\
\hline$\exists 4$ & H cward & 315570 & $10 \%$ & $\exists, \exists \square$ & $\$ 106$ & \$7x 7 1 \\
\hline 活 & Huntington & 259,722 & $35 \%$ & 8,981 & $\$ 106$ & 3951974 \\
\hline$\pi$ & Jeckson & 305,752 & $109 \%$ & $\exists 9, \exists \oplus$ & $\$ 106$ & $5,599,26$ \\
\hline 37 & Jasper & 234,396 & $53 \%$ & 12,516 & $\$ 106$ & $\$ 1,326,718$ \\
\hline 3 & Jay & 249561 & $2.5 \%$ & 6237 & $\$ 106$ & 9661,134 \\
\hline 39 & Jafferson & $\begin{array}{l}163,897 \\
\end{array}$ & $9.1 \%$ & 14,990 & $\$ 106$ & $\$ 1,580928$ \\
\hline 40 & Jennings & 216,726 & $19.1 \%$ & 41,470 & $\$ 106$ & $64,395,828$ \\
\hline 41 & Johnख्र & 270,157 & $26 \%$ & 7020 & $\$ 106$ & \$744091 \\
\hline 42 & Knox & 336,578 & $25.5 \%$ & B5.952 & $\$ 106$ & 9.110932 \\
\hline 43 & Kosciueto & 143594 & $0.3 \%$ & 475 & $\$ 105$ & 50,390 \\
\hline 44 & LaG range & 94,056 & $08 \%$ & 714 & $\$ 106$ & \$75,722 \\
\hline 45 & Lake & 649,440 & $100 \%$ & 64,720 & $\$ 10 E$ & $5,260,3 B$ \\
\hline 46 & LaPorta & 186,102 & $48 \%$ & 9007 & $\$ 106$ & $\$ 954,781$ \\
\hline
\end{tabular}

\begin{tabular}{|c|c|c|c|c|c|c|}
\hline $\operatorname{Code}$ & County & $\begin{array}{l}\text { Area All } \\
\text { Bildges } \\
\text { (sq. ft) }\end{array}$ & $\begin{array}{r}\text { Percent } \\
\text { with S.R. } \\
0.49 .9\end{array}$ & $\begin{array}{r}\text { Area B ridges } \\
\text { with SR } \\
0.49 .9 \\
\text { (sq. ft) }\end{array}$ & $\begin{array}{r}\text { Unit cost } \\
\text { Brildge } \\
\text { Repl acemeint } \\
\text { g/sq. ft. }\end{array}$ & $\begin{array}{r}\text { Total cost } \\
\text { Bridge } \\
\text { Replacement } \\
2002 \text { dollars }\end{array}$ \\
\hline $4 \pi$ & Lawrence & 301,309 & $20.1 \%$ & 60,679 & $\$ 10 \mathrm{~B}$ & $\$ 6,432,016$ \\
\hline $4 \theta$ & Madison & 624,004 & $3.7 \%$ & 22,909 & $\$ 106$ & $\$ 2,428,356$ \\
\hline 49 & Manon & $3,5 \mathrm{~B} 8,54 \mathrm{~B}$ & $5.2 \%$ & 197,940 & $\$ 10 \mathrm{~B}$ & $\$ 19,921,93 \mathrm{~B}$ \\
\hline 50 & M arshall & 314,775 & $0.2 \%$ & 424 & $\$ 106$ & $\$ 44,913$ \\
\hline 51 & Martin & 54,717 & $31.4 \%$ & 17.188 & $\$ 10 \mathrm{~B}$ & $51,822,942$ \\
\hline 52 & Miami & 157,337 & $9.9 \%$ & 25,394 & $\$ 10 \bar{E}$ & $\$ 2,621,765$ \\
\hline 53 & Monroe & 224,947 & $9.6 \%$ & 21,574 & $\$ 106$ & b2, 286,816 \\
\hline 54 & Mantgomery & 387,773 & $4.1 \%$ & 16,123 & $\$ 10 \mathrm{~B}$ & $\$ 1,709,084$ \\
\hline 55 & Morgen & 246,962 & $16.1 \%$ & 39,677 & $\$ 106$ & $54,226,947$ \\
\hline 56 & Nenton & 168,500 & B. $4 \%$ & 14,220 & $\$ 10 \mathrm{~B}$ & $\$ 1,507,272$ \\
\hline 57 & Nable & 90,311 & $12.3 \%$ & 11,191 & $\$ 106$ & $\$ 1,179,906$ \\
\hline 58 & Ohlo & 29,214 & $4.1 \%$ & 1,212 & $\$ 106$ & E128.441 \\
\hline 59 & Orange & $19 \theta, 242$ & $23.7 \%$ & 32,746 & $\$ 106$ & $\$ 3,471,136$ \\
\hline 60 & Owen & 192,777 & $18.2 \%$ & 35,176 & $\$ 105$ & $63,728,703$ \\
\hline 日1 & ParkP & $3 \mathrm{~B} 4,373$ & $24.5 \%$ & 09,614 & $\$ 10 \mathrm{~B}$ & $\$ 7,379,094$ \\
\hline 62 & Perry & 115,255 & 9. $3 \%$ & $10,67 \theta$ & $\$ 106$ & $\$ 1,131,854$ \\
\hline 93 & Plke & 126,100 & $15.0 \%$ & $18,8 \mathrm{B5}$ & $\$ 10 \mathrm{~B}$ & {$[2,001,930$} \\
\hline 64 & Porter & 397,531 & 6. $2 \%$ & 14,687 & $\$ 106$ & $\$ 1,566,852$ \\
\hline 95 & Posey & 212,793 & $24.1 \%$ & $51,2 \mathrm{B7}$ & $\$ 10 \mathrm{~B}$ & [5, 437,491 \\
\hline 68 & Pulaski & $180, \mathrm{~B} 24$ & $5.6 \%$ & 10,067 & $\$ 10 \mathrm{~B}$ & $\$ 1,067,052$ \\
\hline 67 & Putnam & 339,043 & $35.0 \%$ & 118.750 & $\$ 105$ & $\$ 12,687,455$ \\
\hline 68 & Randol中h & $374, \mathrm{~B} 4 \mathrm{~A}$ & B. $0 \%$ & 29,849 & $\$ 10 \mathrm{~B}$ & $\$ 3,163,96 \mathrm{~B}$ \\
\hline 59 & Ripley & 179,803 & $12.4 \%$ & 22,370 & \$105 & $62,371,222$ \\
\hline 70 & RuEh & $2 B 5,189$ & $10.3 \%$ & $27,3 \mathrm{~B} 1$ & $\$ 10 \mathrm{~B}$ & $\$ 2,902,432$ \\
\hline 71 & 51. Joseph & 544,218 & $20.8 \%$ & 113,454 & $\$ 105$ & 51 $2,026,144$ \\
\hline 72 & Scott & $\mathrm{B} 3, \mathrm{~B} 4 \mathrm{G}$ & B.T\% & 4,302 & $\$ 106$ & $\$ 450,046$ \\
\hline 73 & Shelby & 382,606 & $10.7 \%$ & 40,844 & $\$ 106$ & $64,329,440$ \\
\hline 74 & Epenc er & $163,31 \mathrm{~g}$ & $5.8 \%$ & 9,514 & $\$ 10 \mathrm{~B}$ & $61,008,480$ \\
\hline 75 & Starke & 82,504 & $25.9 \%$ & 21,362 & $\$ 106$ & $52,264,394$ \\
\hline 76 & steuban & 67,351 & $0.0 \%$ & 0 & $\$ 10 \mathrm{~B}$ & 80 \\
\hline 77 & Sulliran & $32 \theta, 949$ & $17.3 \%$ & 39,583 & $\$ 106$ & $\$ 4,195,773$ \\
\hline 78 & Swtrenand & 52,321 & $25.6 \%$ & 13,940 & $\$ 105$ & E1, 477,683 \\
\hline 79 & Tippecanoe & 473,295 & $2.0 \%$ & 13,016 & $\$ 106$ & $\$ 1,379,664$ \\
\hline 80 & Tipton & 126,345 & $2.2 \%$ & 2,815 & काण & 6298.462 \\
\hline 91 & Unian & 94,462 & $4.3 \%$ & 4,047 & $\$ 106$ & $\$ 428,980$ \\
\hline$\theta 2$ & Vanderburgh & 434,941 & $0.5 \%$ & 2,380 & $\$ 106$ & E253,13日 \\
\hline 93 & Vermillion & 160,651 & $11.5 \%$ & 18,510 & $\$ 10 \mathrm{~B}$ & $\$ 1,962,040$ \\
\hline 日4 & Vigo & 344,329 & $5.3 \%$ & 18.179 & $\$ 106$ & 61.926,963 \\
\hline 95 & Wabash & 336,005 & $5.6 \%$ & 18,932 & $\$ 10 \mathrm{~B}$ & $\$ 2,000,93 \mathrm{~B}$ \\
\hline 86 & Warren & 173,663 & $22.7 \%$ & 39.420 & $\$ 106$ & $54,178,520$ \\
\hline at & warrlck & 219,333 & $7.8 \%$ & 17,046 & $\$ 10 \mathrm{~B}$ & $\$ 1,800,930$ \\
\hline $8 \theta$ & Washington & 309,623 & $2.0 \%$ & 5,890 & $\$ 106$ & $\{\in 24,360$ \\
\hline 98 & Wayne & 629,197 & $7.2 \%$ & 45,081 & $\$ 10 \mathrm{~B}$ & $64,779,055$ \\
\hline 90 & Wellg & 316,276 & $4.6 \%$ & 9,982 & $\$ 106$ & $\$ 1,058,129$ \\
\hline 91 & whitt & 323,734 & $0.4 \%$ & 1,216 & $\$ 106$ & 6129,974 \\
\hline 92 & Whilley & 124,323 & B. $3 \%$ & 10,329 & $\$ 10 \mathrm{E}$ & $\$ 1,094,918$ \\
\hline
\end{tabular}


Appendix K. Cost Estimations for Local Bridge Rehabilitation

\begin{tabular}{|c|c|c|c|c|c|c|}
\hline $\operatorname{Cod} \theta$ & County & $\begin{array}{l}\text { Area All } \\
\text { Bildges } \\
\text { (sq. fi) }\end{array}$ & $\begin{array}{r}\text { Percent } \\
\text { whth S.R. } \\
50.79 .9\end{array}$ & $\begin{array}{r}\text { Area Brldges } \\
\text { whth SR } \\
50-79.9 \\
\text { (sq. ft] }\end{array}$ & $\begin{array}{r}\text { Unit cost } \\
\text { Bildge } \\
\text { Rehabh hhatlen } \\
\$ / \delta q . \text { fi. }\end{array}$ & $\begin{array}{r}\text { Total cost } \\
\text { Bridge } \\
\text { Rehabbllliatlon } \\
2002 \text { dollars }\end{array}$ \\
\hline 1 & Adams & 229544 & $0.6 \%$ & $19, \theta 0 \mathrm{~B}$ & $\$ 30$ & $\$ 594229$ \\
\hline 2 & Alløп & $1,077,067$ & $36.8 \%$ & 396,491 & $\$ 30$ & $\$ 11,094735$ \\
\hline 3 & Ba thabmew & 574,700 & $19.6 \%$ & $112,3 \oplus$ & $\$ 30$ & 幽, $371 \% 7$ \\
\hline 4 & Benton & $1 \mathrm{G}, 412$ & $5.7 \%$ & 9,275 & 530 & $\$ 278,249$ \\
\hline 5 & Blackiford & 79662 & $6.0 \%$ & 4,747 & $\$ 30$ & $\$ 142,411$ \\
\hline 6 & Boone & 258524 & $11.8 \%$ & $35,3 \mathrm{E} 5$ & $\$ 30$ & $\$ 1,060965$ \\
\hline 7 & Brown & $\mathrm{E} 6,153$ & $30.6 \%$ & 26,021 & $\$ 30$ & $\$ 780529$ \\
\hline$\theta$ & Caाroll & 255,953 & $22.8 \%$ & 58,442 & $\$ 30$ & $\$ 1,753257$ \\
\hline 9 & Cass & 287680 & $2.4 \%$ & 6,997 & $\$ 30$ & $\$ 209907$ \\
\hline 10 & Clark & 20518 & $13.2 \%$ & $26,4 \mp$ & 530 & \$790 D75 \\
\hline 11 & Clay & 1 㕅 913 & $29.8 \%$ & 55,334 & 530 & $\$ 1,6 \mathbf{D}^{\circ} \mathrm{g}$ \\
\hline 12 & Clinton & 247231 & $22.1 \%$ & $54,7 \mathrm{BO}$ & $\mathbf{5 3 0}$ & $\$ 1.642789$ \\
\hline 13 & Crawford & 101,434 & $45.8 \%$ & 46.472 & $\$ 30$ & $\$ 1,394,167$ \\
\hline 14 & Daxiass & 171,747 & $9.0 \%$ & 15,442 & $\$ 90$ & $\$ 463247$ \\
\hline 15 & Dearborn & 155,062 & $27.0 \%$ & 52,595 & $\$ 30$ & $\$ 1,577 \mathrm{~d} d 7$ \\
\hline 16 & Dec atur & $25 \overline{5}, 779$ & $25.1 \%$ & 64,211 & $\$ 30$ & $\$ 1,926790$ \\
\hline 17 & 口ekalb & 197,732 & $2.7 \%$ & 5,312 & 530 & \$1田王6 \\
\hline 18 & Delaware & 575,867 & $16.8 \%$ & 96,534 & 530 & 农, 晒口27 \\
\hline 19 & Dubois & 191.729 & $14.0 \%$ & $26.8 \mathrm{LO}$ & $\mathbf{8 3 0}$ & $\$ 804068$ \\
\hline 20 & Elkhart & $497.47 \mathrm{~B}$ & $30.4 \%$ & 151.129 & $\mathbf{5 3 0}$ & $\$ 4,533 \$ 69$ \\
\hline 21 & Fayet1e & $15987 \mathrm{~B}$ & $5.4 \%$ & 10.436 & $\$ 30$ & $\$ 313092$ \\
\hline 22 & Floyd & 142,092 & $12.4 \%$ & $17,5 \mathrm{Bg}$ & $\$ 30$ & $\$ 527 反 600$ \\
\hline $2 \exists$ & Fountain & 2西 D78 & $21.5 \%$ & 48,67 & 530 & $\$ 1,49054$ \\
\hline 24 & Franklin & 고크 0 코 & $16.2 \%$ & 37,644 & 530 & $\$ 1,1]$ ב \\
\hline 25 & Fulton & 110597 & $5.5 \%$ & 5,16 & 530 & $\$ 180,173$ \\
\hline $2 \mathrm{~B}$ & Gibson & 250671 & $35.9 \%$ & 104.456 & $\mathbf{8 3 0}$ & $\$ 3,133692$ \\
\hline 27 & Grant & 359,872 & $20.2 \%$ & 68.459 & $\$ 30$ & $\$ 2,054901$ \\
\hline $2 \mathrm{~B}$ & Gre ene & 207633 & $15.7 \%$ & 32,695 & $\$ 30$ & $\$ 980663$ \\
\hline 29 & Hamilton & 632,155 & $11.1 \%$ & $70,3 \mathrm{Bg}$ & $\$ 30$ & $\$ 2,111685$ \\
\hline 30 & Hencock & 279554 & $\theta .1 \%$ & 27,573 & 530 & \$Бत7, 19日 \\
\hline 31 & Herrison & 13044 & $14.5 \%$ & $18,7 \square$ & 530 & \$551 246 \\
\hline 32 & Hendricks & 426996 & $20.1 \%$ & 85,979 & $\mathbf{8 3 0}$ & $\$ 2,579,347$ \\
\hline 33 & Henry & 211,509 & $8.6 \%$ & 18,659 & $\mathbf{\$ 3 0}$ & $\$ 560781$ \\
\hline 34 & Howard & 315,570 & $38.7 \%$ & 122,050 & $\$ 30$ & $\$ 3,662779$ \\
\hline 35 & Huntington & 259,722 & $27.4 \%$ & 71.100 & $\$ 30$ & $\$ 2,133258$ \\
\hline $3 \mathrm{~B}$ & Jackson & 305,752 & $12.7 \%$ & 38,957 & $\$ 30$ & $\$ 1,168720$ \\
\hline 37 & Јавр日r & 234,396 & $6.2 \%$ & 14,606 & $\$ 90$ & $\$ 479,190$ \\
\hline 3 분 & Jay & 249561 & $18.6 \%$ & 46,367 & $\$ 30$ & $\$ 1,391015$ \\
\hline 39 & Jefferson & 169,897 & $11.1 \%$ & 18.122 & $\$ 30$ & $\$ 543649$ \\
\hline 40 & Jennings & 216,726 & $14.2 \%$ & 30.814 & $\$ 30$ & $\$ 924,431$ \\
\hline 41 & Johnson & 270.157 & $11.5 \%$ & 30,977 & $\$ 30$ & $\$ 929314$ \\
\hline 42 & Knox & $33657 \mathrm{~B}$ & $24.2 \%$ & 91,549 & $\$ 30$ & $\$ 2,446,438$ \\
\hline 43 & Kosciusko & 143594 & $11.0 \%$ & 15,755 & $\$ 30$ & $\$ 472661$ \\
\hline 44 & LaGr ange & 94056 & $6.9 \%$ & 6,500 & $\$ 30$ & $\$ 194907$ \\
\hline 45 & Lake & 649,440 & $22.1 \%$ & 143,251 & 530 & \$4, \\
\hline 46 & LePurte & 1田,102 & $7.1 \%$ & 13,170 & 930 & \$395中田 \\
\hline
\end{tabular}

\begin{tabular}{|c|c|c|c|c|c|c|}
\hline Code & County & 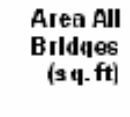 & $\begin{array}{l}\text { Percent } \\
\text { with S.R. } \\
50.79 .9\end{array}$ & $\begin{array}{r}\text { Area Bildges } \\
\text { whth SR } \\
50.799 \\
\text { [s il. ft] }\end{array}$ & $\begin{array}{r}\text { Unit cost } \\
\text { Brldq } \\
\text { Rehobbilitation } \\
\$ / s q . \mathrm{f1} .\end{array}$ & $\begin{array}{r}\text { Total cost } \\
\text { Brldq } \\
\text { Rehabbilliation } \\
2002 \text { dollars }\end{array}$ \\
\hline 47 & Lawrence & 301,359 & $169 \%$ & 44,222 & $\$ 30$ & $\$ 1,326,661$ \\
\hline 48 & Madison & 624,004 & $129 \%$ & 89.256 & $\$ 30$ & $\$ 2,677,672$ \\
\hline 49 & Marion & 3580546 & $422 \%$ & $1,313,556$ & $\$ 90$ & $\$ 99,406,665$ \\
\hline 50 & Marshall & 214,775 & $4.5 \%$ & 8,249 & 530 & 5247,440 \\
\hline 51 & Martin & 54,717 & $362 \%$ & 14,457 & $\$ 30$ & $\$ 433,720$ \\
\hline 52 & Miami & 257,337 & $239 \%$ & 44 OEE & $\$ 30$ & $\$ 1,321,949$ \\
\hline 53 & Monrae & 234947 & $25.4 \%$ & 38,342 & 530 & $\$ 1,190$ \\
\hline 54 & Montgomery & 397,773 & $8.1 \%$ & 27,076 & $\$ 30$ & $\$ 812,268$ \\
\hline 55 & Morgan & $24 \mathrm{E} 9 \mathrm{E} 2$ & $206 \%$ & 46.187 & $\$ 30$ & $\$ 1,386,609$ \\
\hline 56 & Newrtan & 16855 & $190 \%$ & 36876 & 930 & $\$ 1,106,297$ \\
\hline 57 & Noble & 90,311 & $200 \%$ & 10,519 & 530 & 5315,56 \\
\hline 58 & Ohio & 29214 & $182 \%$ & 1,049 & 830 & 631,436 \\
\hline 59 & Drange & 130,242 & $198 \%$ & 17934 & 930 & $35 \%, 000$ \\
\hline E口 & Dwen & 192,777 & $17 \square \%$ & 1967 & 530 & 95田昌 7 \\
\hline 51 & Parke & 284,373 & $34.1 \%$ & $71,3 \mathrm{BD}$ & 530 & $52,141,399$ \\
\hline 62 & Perry & 115,256 & $35.3 \%$ & 30950 & $\$ 30$ & $\$ 929,092$ \\
\hline 63 & Fikg & $12 \mathrm{~B}, 106$ & $22.3 \%$ & $22 ; 5 \mathrm{BD}$ & $\$ 90$ & $\$ 677,563$ \\
\hline 64 & Porter & 237531 & उБ9\% & 日2070 & 530 & $52,4 \square, 101$ \\
\hline 65 & Posey & 212,750 & $16.9 \%$ & 24,792 & $\$ 30$ & $\$ 743,758$ \\
\hline 66 & Pulaski & 180,824 & $5.3 \%$ & 5,377 & $\$ 30$ & $\$ 161 ; 310$ \\
\hline 67 & Putnam & 339043 & $200 \%$ & 52 D每 & $\$ 30$ & $\$ 1562596$ \\
\hline 68 & Randalph & 374,649 & $16.4 \%$ & 42,933 & $\$ 30$ & $\$ 1287978$ \\
\hline 69 & Ripley & $1798 \mathrm{BCO}$ & $27.5 \%$ & $32,7 \mathrm{BO}$ & $\$ 30$ & $\$ 983,405$ \\
\hline 70 & Ruন্ধ & 265,189 & $212 \%$ & $52,5 \mathrm{BE}$ & $\$ 30$ & $\$ 15775 \mathrm{dA}$ \\
\hline 71 & S1. Joseph & 544218 & $22.7 \%$ & 170,414 & $\$ 30$ & $\$ 5,112,406$ \\
\hline 72 & Scott & 63846 & $25.4 \%$ & 17,825 & $\$ 30$ & $\$ 534,765$ \\
\hline 73 & Shalby & $382,6 \mathrm{DG}$ & $249 \%$ & 61033 & $\$ 30$ & $\$ 1,830,984$ \\
\hline 74 & Spencer & $16 \boxminus 318$ & $272 \%$ & 34,719 & 530 & 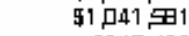 \\
\hline 75 & Sarke & 82,504 & $33.7 \%$ & $28.1 \mathrm{Bg}$ & 830 & 9845,480 \\
\hline 76 & Seuben & 67,351 & $42 \%$ & 3,419 & $\$ 30$ & $\$ 102,562$ \\
\hline 77 & Sulivan & 228949 & $399 \%$ & $54,4 \subset \mathrm{CO}$ & 930 & $\$ 1,632,096$ \\
\hline 78 & Switzerland & 52,321 & $27.5 \%$ & 9,687 & 530 & 5290,615 \\
\hline 79 & Tippecanoe & 473295 & $16.5 \%$ & 64,325 & 830 & $\$ 1,929,748$ \\
\hline 80 & Tipron & 126,346 & 139 & 8,030 & $\$ 30$ & $\$ 240,888$ \\
\hline 1 & Unian & 94,46 & $115 \%$ & 4,211 & 530 & 51 五 344 \\
\hline 82 & Vanderburgh & 434,941 & $22.4 \%$ & $118,6 \mathrm{~B} 7$ & 530 & 53500601 \\
\hline 83 & Varmilian & 160,651 & $160 \%$ & 23,429 & $\$ 30$ & $\$ 702,866$ \\
\hline 64 & vigo & 344,929 & $262 \%$ & 65,480 & $\$ 30$ & $\$ 1,969,607$ \\
\hline 85 & Wobash & 336 & $13 \mathrm{\theta} \%$ & 430 & 530 & 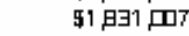 \\
\hline 86 & Warren & $1736 \mathrm{EG}$ & $16.5 \%$ & 21.917 & 330 & 3657901 \\
\hline$\theta 7$ & Warrick & 219,939 & $10 \theta \%$ & 34,280 & $\$ 30$ & $\$ 1,028,405$ \\
\hline 殗 & Washingtan & 209629 & $21.4 \%$ & 30643 & $\$ 30$ & $\$ 919780$ \\
\hline 89 & Wayne & 629.197 & $170 \%$ & 123,750 & 530 & $53,712,491$ \\
\hline 90 & Walls & 216276 & $19.5 \%$ & 31,823 & $\$ 30$ & $\$ 954,682$ \\
\hline 91 & White & 323,734 & $8.9 \%$ & 29013 & $\$ 30$ & \$970; 79 \\
\hline 97 & Whitley & 124,320 & $405 \%$ & 41,481 & 530 & $51,244,475$ \\
\hline
\end{tabular}


Appendix L. Summary of Local Community Highway Projects

Added Travel Lanes

Bike/Pedestrian Facilities

Bridge Replacement

Bridge Removal

Bridge Widening

Bridge Deck Reconstruction

Bridge deck reconstruction and Widening

Bridge Deck Replacement

Bridge Repair or Rehabilitation

Covered Bridge rehabilitation

Demolition

Enhancement

Buildings

Environmental Mitigation

Historical site Preservation

Intersect. Improv. W/ Added Turn Lanes

Intersect. Improv. W/ New Signals

Intersection Improvement

Its Traffic Monitoring

Landscaping

new Bridge Construction

New Road Construction

New Signal Installation

Other Project type

Pavement Rehabilitation

Pavement replacement

Railroad Crossing

Railroad Protection

railroad work

Replace Superstructure

Resurface

Road Construction

Road Reconstruction

Roadside work

Scenic Easements

Sight Distance Improvement

Signs, Lighting, signals and markings

Small Structure Replacement

Traffic signals

Truss Reconstruction or Repair

Utility Relocation

Widen Pavement and Bit Overlay

Total

\begin{tabular}{|c|c|c|c|c|c|c|c|c|}
\hline 1999 & 2000 & 2001 & 2002 & 2003 & 2004 & 2005 & 2006 & 2007 \\
\hline & & & & $37,559.00$ & $6,649.00$ & $9,103.00$ & $16,400.00$ & $13,210.00$ \\
\hline & & & & $9,714.00$ & $10,398.00$ & $4,080.00$ & & \\
\hline & & & 300.00 & $34,406.00$ & $45,034.00$ & $35,357.00$ & $29,999.00$ & $2,773.00$ \\
\hline & & & & & & 155.00 & 450.00 & \\
\hline & & & & $2,461.00$ & $2,742.00$ & & 800.00 & \\
\hline & & & & & & & 990.00 & \\
\hline & & & & & $6,166.00$ & & & \\
\hline & & & & & & & $2,610.00$ & $1,200.00$ \\
\hline & & & $2,461.00$ & $10,788.00$ & $5,051.00$ & $12,307.00$ & $6,387.00$ & 900.00 \\
\hline & & & & $3,637.00$ & $1,288.00$ & 600.00 & & \\
\hline & & & & 685.00 & & & & \\
\hline $1,376.00$ & 252.00 & 300.00 & 998.00 & $30,221.00$ & $19,108.00$ & $139,074.00$ & 625.00 & \\
\hline & & & & & $2,630.00$ & & & \\
\hline & & & & 500.00 & & & & \\
\hline & & & & $4,113.00$ & & 438.00 & & \\
\hline & & & & 700.00 & $1,770.00$ & 315.00 & $1,600.00$ & 325.00 \\
\hline & & & & $4,410.00$ & & & & \\
\hline & & & $3,247.00$ & $9,943.00$ & $7,332.00$ & $7,230.00$ & 780.00 & $1,000.00$ \\
\hline & & & 50.00 & & & & & \\
\hline & & & & & $2,300.00$ & & & \\
\hline & & & & $16,974.00$ & 750.00 & $6,520.00$ & $1,345.00$ & $1,020.00$ \\
\hline & & & & $41,239.00$ & $15,961.00$ & $15,844.00$ & $32,561.00$ & $26,201.00$ \\
\hline & & & & 75.00 & 40.00 & & & \\
\hline & $2,861.00$ & $1,878.00$ & $9,532.00$ & $6,995.00$ & $4,287.00$ & 625.00 & & 5.00 \\
\hline & & & $8,558.00$ & $68,013.00$ & $65,684.00$ & $1,114,899.00$ & $28,803.00$ & $14,220.00$ \\
\hline & & & & $2,999.00$ & $9,370.00$ & $7,428.00$ & $5,051.00$ & \\
\hline & & 100.00 & 200.00 & 330.00 & 500.00 & 850.00 & 250.00 & \\
\hline & 912.00 & $1,934.00$ & $1,100.00$ & $1,016.00$ & 100.00 & 200.00 & 5.00 & \\
\hline & & & $7,231.00$ & & $1,200.00$ & $1,020.00$ & & \\
\hline & & & & & & & 249.00 & \\
\hline & & & & $2,376.00$ & 195.00 & 0.00 & 450.00 & \\
\hline & & & & $5,837.00$ & $18,647.00$ & $29,513.00$ & 0.00 & $8,150.00$ \\
\hline & & & & $16,345.00$ & $18,855.00$ & $24,711.00$ & $19,505.00$ & $28,364.00$ \\
\hline & & & & 494.00 & $1,231.00$ & 150.00 & & \\
\hline & & & & & 300.00 & 804.00 & & \\
\hline & & & & & 415.00 & & & \\
\hline & & & & $1,010.00$ & 833.00 & $3,131.00$ & & \\
\hline & & & & 200.00 & & & & \\
\hline & & & & $6,689.00$ & $3,875.00$ & $2,409.00$ & 813.00 & \\
\hline & & & & 428.00 & & & & \\
\hline & & & & 320.00 & & & & \\
\hline & & & & 525.00 & $2,000.00$ & & & \\
\hline & & & & 100200 & 71100 & 676 & 967300 & \\
\hline
\end{tabular}


Appendix L. Summary of Local Community Highway Projects (cont'd)

Added Travel Lanes
Bike/Pedestrian Facilities
Bridge Replacement
Bridge Removal
Bridge Widening
Bridge Deck Reconstruction
Bridge deck reconstruction and Widening
Bridge Deck Replacement
Bridge Repair or Rehabilitation
Covered Bridge rehabilitation
Demolition
Enhancement
Buildings
Environmental Mitigation
Historical site Preservation
Intersect. Improv. W/ Added Turn Lanes
Intersect. Improv. W/ New Signals
Intersection Improvement
Its Traffic Monitoring
Landscaping
new Bridge Construction
New Road Construction
New Signal Installation
Other Project type
Pavement Rehabilitation
Pavement replacement
Railroad Crossing
Railroad Protection
railroad work
Replace Superstructure
Resurface
Road Construction
Road Reconstruction
Roadside work
Scenic Easements
Sight Distance Improvement
Signs, Lighting, signals and markings
Small Structure Replacement
Traffic signals
Truss Reconstruction or Repair
Utility Relocation
Widen Pavement and Bit Overlay
Total

\begin{tabular}{|c|c|c|c|c|c|c|c|c|c|}
\hline 2008 & 2009 & $\begin{array}{l}\mathbf{2 0 1 0} \\
2,293.00\end{array}$ & 2011 & 2012 & 2013 & 2014 & 2015 & 2016 & $\begin{array}{l}\text { Total } \\
85,214.00\end{array}$ \\
\hline & & 190.00 & & & & & & & $24,382.00$ \\
\hline 350.00 & & & & & $1,300.00$ & & & & $149,519.00$ \\
\hline & & & & & & & & & $\begin{array}{r}605.00 \\
6.003 .00\end{array}$ \\
\hline & & & & & & & & & $\begin{array}{r}6,003.00 \\
990.00\end{array}$ \\
\hline & & & & & & & & & $6,166.00$ \\
\hline & & & & & & & & & $3,810.00$ \\
\hline & & & & & & & & & $37,894.00$ \\
\hline & & & & & & & & & $5,525.00$ \\
\hline & & & & & & & & & 685.00 \\
\hline & & & & & & & & & $191,954.00$ \\
\hline & & & & & & & & & $2,630.00$ \\
\hline & & & & & & & & & 500.00 \\
\hline & & & & & & & & & $4,551.00$ \\
\hline & & & & & & & & & $4,710.00$ \\
\hline & & & & & & & & & $4,410.00$ \\
\hline & & & & & & & & & $29,532.00$ \\
\hline & & & & & & & & & 50.00 \\
\hline & & & & & & & & & $2,300.00$ \\
\hline & & $1,595.00$ & & & & & & & $28,204.00$ \\
\hline & $1,000.00$ & & & & 814.00 & & $2,400.00$ & $25,000.00$ & $161,020.00$ \\
\hline & & & & & & & & & 115.00 \\
\hline & & & & & & & & & $26,183.00$ \\
\hline & & $1,923.00$ & & & $1,990.00$ & & & & $1,304,090.00$ \\
\hline & & & & & & & & & $24,848.00$ \\
\hline & & & & & & & & & $2,230.00$ \\
\hline & & & & & & & & & $5,267.00$ \\
\hline & & & & & & & & & $9,451.00$ \\
\hline & & & & & & & & & 249.00 \\
\hline & & & & & & & & & $3,021.00$ \\
\hline 0.00 & 0.00 & $3,000.00$ & & & & & & & $65,147.00$ \\
\hline 0.00 & 0.00 & 0.00 & 0.00 & & & & & & $107,780.00$ \\
\hline & & & & & & & & & $1,875.00$ \\
\hline & & & & & & & & & $1,104.00$ \\
\hline & & & & & & & & & 415.00 \\
\hline & & & & & & & & & $4,974.00$ \\
\hline & & & & & & & & & 200.00 \\
\hline & & & & & & & & & $13,786.00$ \\
\hline & & & & & & & & & 428.00 \\
\hline & & & & & & & & & 320.00 \\
\hline & & & & & & & & & $2,525.00$ \\
\hline & & & & & & & & & 0.00 \\
\hline 350.00 & $1,000.00$ & $9,001.00$ & 0.00 & 0.00 & $4,104.00$ & 0.00 & $2,400.00$ & $25,000.00$ & $2,324,662.00$ \\
\hline
\end{tabular}


Appendix M. Fuel Efficiency by Model Year (miles per gallon)

\begin{tabular}{|c|c|c|c|}
\hline Year & Automobiles & $\begin{array}{c}\text { Light Duty } \\
\text { Trucks }\end{array}$ & Tractors \\
\hline 1988 & 26 & 15.4 & 5.8 \\
\hline 1989 & 26.5 & 16.1 & 5.8 \\
\hline 1990 & 27.5 & 16.1 & 5.8 \\
\hline 1991 & 27.5 & 17 & 5.7 \\
\hline 1992 & 27.5 & 17.3 & 5.8 \\
\hline 1993 & 28 & 17.4 & 5.8 \\
\hline 1994 & 28 & 17.3 & 5.8 \\
\hline 1995 & 28 & 17.3 & 5.8 \\
\hline 1996 & 28 & 17.2 & 5.9 \\
\hline 1997 & 28 & 17.2 & 6.1 \\
\hline 1998 & 28.3 & 17.2 & 6.1 \\
\hline 1999 & 28.3 & 17 & 5.4 \\
\hline 2000 & 28.3 & 17.5 & 5.3 \\
\hline 2001 & 28.7 & 17.6 & 5.3 \\
\hline 2002 & 28.7 & 17.8 & 5.4 \\
\hline 2003 & 28.7 & 17.9 & 5.4 \\
\hline 2004 & 28.7 & 18.1 & 5.5 \\
\hline 2005 & 28.7 & 18.2 & 5.5 \\
\hline 2006 & 30.9 & 18.4 & 5.6 \\
\hline 2007 & 31.3 & 18.5 & 5.6 \\
\hline 2008 & 31.7 & 18.7 & 5.6 \\
\hline 2009 & 31.7 & 18.7 & 5.6 \\
\hline 2010 & 31.7 & 18.7 & 5.6 \\
\hline 2011 & 31.7 & 18.7 & 5.6 \\
\hline 2012 & 31.7 & 18.7 & 5.6 \\
\hline 2013 & 31.7 & 18.7 & 5.6 \\
\hline 2014 & 31.7 & 18.7 & 5.6 \\
\hline 2015 & 31.7 & 18.7 & 5.6 \\
\hline 2016 & 31.7 & 18.7 & 5.6 \\
\hline 2017 & 31.7 & 18.7 & 5.6 \\
\hline 2018 & 31.7 & 18.7 & 5.6 \\
\hline 2019 & 31.7 & 18.7 & 5.6 \\
\hline 2020 & 31.7 & 18.7 & 5.6 \\
\hline
\end{tabular}

Values for 1988-2002 from Transportation Energy Data Book (ORNL-6970, 2003)

Values for 2003-2020 estimated 